\title{
Integration of Reaction Kinetics Theory and Gene Expression Programming to Infer Reaction Mechanism
}

Jason R. White

jason.white@uconn.edu

Follow this and additional works at: https://opencommons.uconn.edu/dissertations

\section{Recommended Citation}

White, Jason R., "Integration of Reaction Kinetics Theory and Gene Expression Programming to Infer Reaction Mechanism" (2014). Doctoral Dissertations. 371.

https://opencommons.uconn.edu/dissertations/371 
Integration of Reaction Kinetics Theory and Gene Expression Programming to Infer

\author{
Reaction Mechanism \\ Jason Robert White, $\mathrm{PhD}$ \\ University of Connecticut, 2014
}

\begin{abstract}
Mechanistic mathematical models of biological systems have been used to describe biological phenomena, including human disease, in the hope that one day these models may be used to better understand diseases, as well as to develop and optimize therapeutic strategies. Evolutionary algorithms, such as genetic programming, may be used to symbolically regress mathematical models describing chemical and biochemical species for which kinetic data are available. However, current evolutionary algorithms are restricted to the formulation of simple or approximate models due to the computational cost of evolving mechanistic models for more complex systems.

It was hypothesized that chemical reaction kinetic theory could be used to sufficiently reduce the model search space for an evolutionary algorithm such that it would be possible to infer mechanistic mathematical models of complex biological interactions. An evolutionary algorithm capable of formulating mass action kinetic models of biological systems from time series data sets was developed for a system of nspecies using heuristics from chemical reaction kinetic theory and a gene expression programming (GEP) based approach.
\end{abstract}


Jason Robert White - University of Connecticut, 2014

The resulting algorithm was then successfully validated on a general model of viral dynamics that accounted for six pathways relating the change in viral template, viral genome, and viral structural protein concentrations over time.

The algorithm was applied to generate cohort-specific models of HIV dynamics from a clinical data set. HIV-1 infection models were defined as sets of two ordinary differential equations describing the change in $\mathrm{CD}^{+}{ }^{+}$T-cell and HIV-1 concentrations over time. The evolved models were used to generate hypotheses regarding treatment effectiveness and the potential for viral rebound in three cohorts of HIV-1 positive individuals receiving different Highly Active Antiretroviral Therapy (HAART) regimens. It was hypothesized by the algorithm that HAART was effective in stopping HIV-1 propagation in two of the three cohorts studied. In the other cohort, it was hypothesized that HIV-1 continued to propagate and that there was the potential for viral rebound.

The result of this work was the development of an algorithm that can be used for the generation of complex mechanistic biological models based upon kinetic data with potential uses in fields ranging from biomedical to biotechnological. 
Integration of Reaction Kinetics Theory and Gene Expression Programming to Infer Reaction Mechanism

\author{
Jason Robert White
}

B.S., University of Connecticut, 2008

M.S., University of Connecticut, 2013

\author{
A Dissertation \\ Submitted in Partial Fulfillment of the \\ Requirements for the Degree of Doctor of Philosophy \\ at the \\ University of Connecticut
}

2014 
Copyright by

Jason Robert White

2014 


\begin{abstract}
APPROVAL PAGE
Doctor of Philosophy Dissertation

Integration of Reaction Kinetics Theory and Gene Expression Programming to Infer

Reaction Mechanism
\end{abstract}

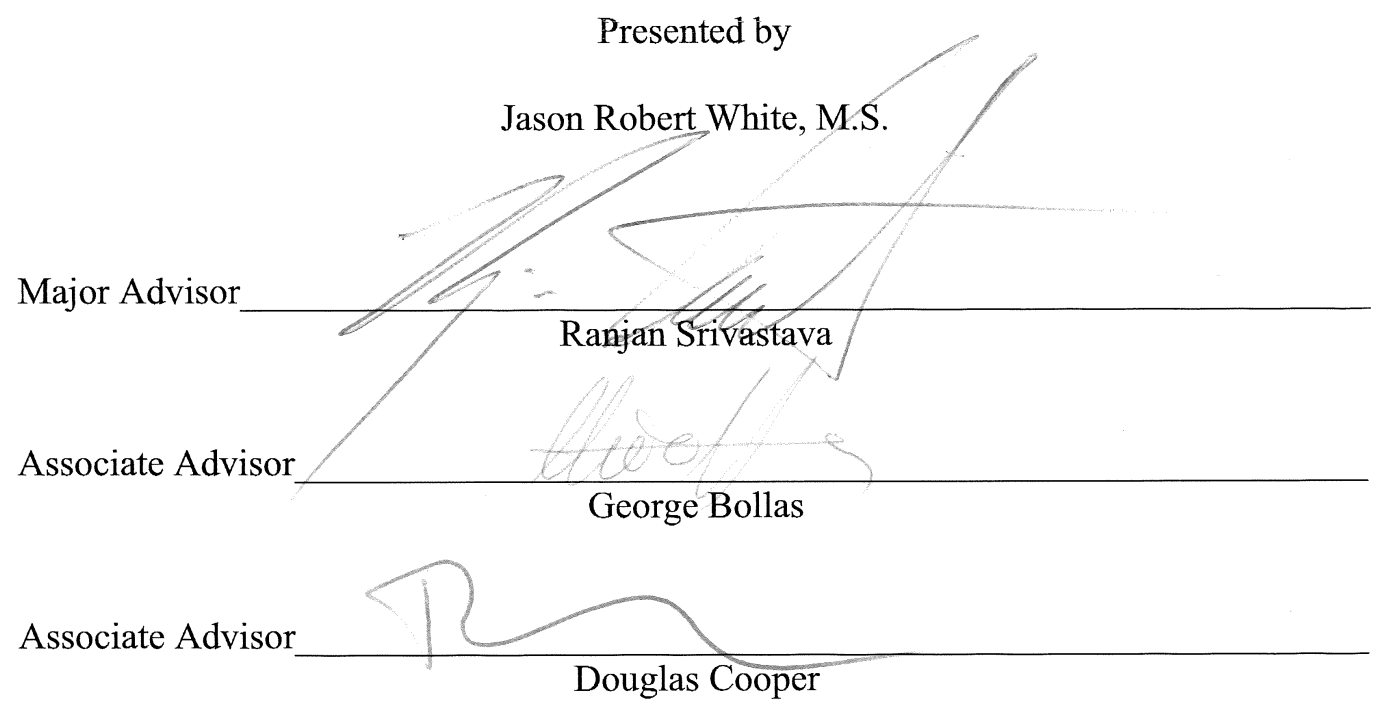

University of Connecticut

2014 


\section{Acknowledgements}

I would like to thank my advisor, Dr. Ranjan Srivastava, for all of his help and guidance in both my research work as well as for his advice in many personal and professional situations. I truly appreciate his encouragement of my ideas and his supporting me in pursuing outreach activities that have enriched my graduate school experience. I would like to thank the members of the Srivastava Lab for their help and support and for fostering an enjoyable work environment. I would like to thank Dr. George Bollas and Ms. Lu Han for assisting with the HAART optimization research discussed in Chapter 4. I am also grateful for collaborations with Dr. Douglas Peterson, Dr. Rajesh Lalla, Dr. Leslie Loew, and Dr. Kevin Dieckhaus from the University of Connecticut Health Center on the Oral Mucositis and HAART optimization research discussed in Chapters 2 and 4. I would like to acknowledge Dr. Doug Cooper, Dr. Kazem Kazerounian, Ms. Aida Ghaiei, and members of the NSF-funded GK-12 program at UCONN for their support and allowing me an opportunity to grow as a teacher. I would also like to thank the members of my committee, Dr. Doug Cooper, Dr. George Bollas, Dr. Daniel Burkey, and Dr. Brian Willis, for their time and support of my research work. Finally, I would like to thank my wife Chelsee White and my daughters Teá and Amaryn for their love and support as I've taken significant time to pursue my interests. You mean everything to me, I couldn't have done this without you, and I am blessed to have you as family. 


\section{Table of Contents}

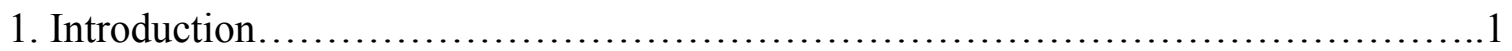

1.1 Motivation......................................................

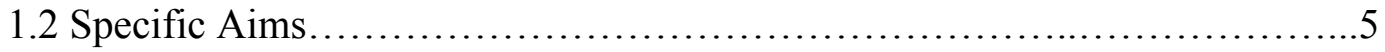

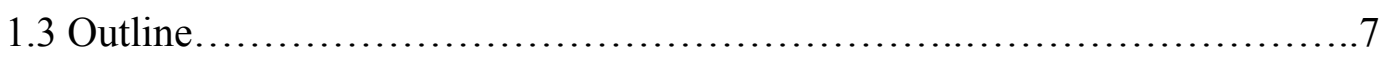

2. Modeling Of Oral Cavity Mucositis..........................................

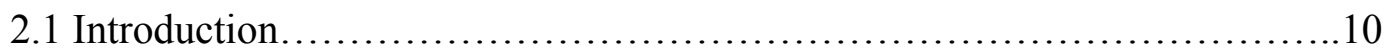

2.2 Methods...................................................... 13

2.3 Results....................................................... 16

2.4 Discussion...................................................... 18

3. Development Of An Elite-Variant Selection Strategy For Genetic Algorithm Optimization.............................................................20

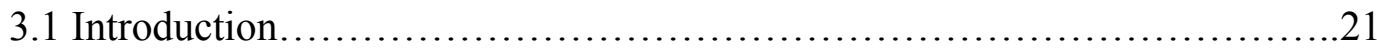

3.2 Methods...................................................... 24

3.2.1 Development of Elite Variant Selection Genetic Algorithm........24

3.2.2 Application of Test Cases to Elite-Variant Selection Genetic

Algorithm............................................28

3.3 Results............................................................. 34

3.3.1 Function Tests..............................................

3.3.2 Satisfiability Test...................................... 38

3.3.3 HAART Optimization......................................40

3.4 Discussion....................................................46

4. Optimizing HIV-1 Treatment Via A Systems Biology Approach...................49 


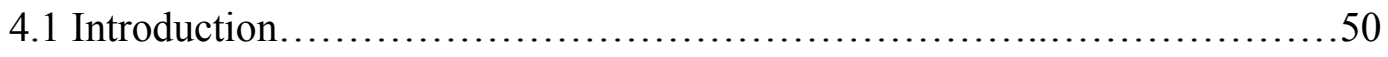

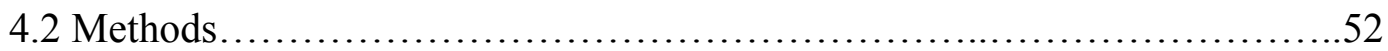

4.2.1 Model Selection............................................52

4.2.2 Optimization of Highly Active Antiretroviral Therapy..............55

4.2.3 Comparison of Optimized HAART to Standard HIV-1 Treatments Administered to Computer-Generated Cohorts...................57

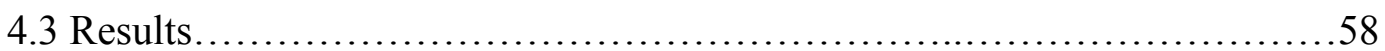

4.3.1 Optimal HAART Dosage for Base Case..........................58

4.3.2 Comparison of HAART Strategies via Simulation Studies...........59

4.3.3 Comparison of Simulation to Experimental STI Results.............61

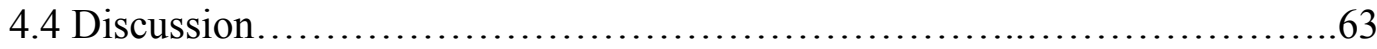

5. Discovery of Biochemical Kinetics and Mechanism Using a GEP Based Evolutionary

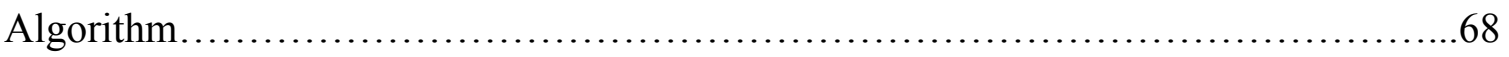

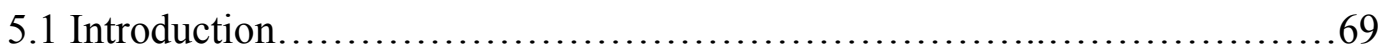

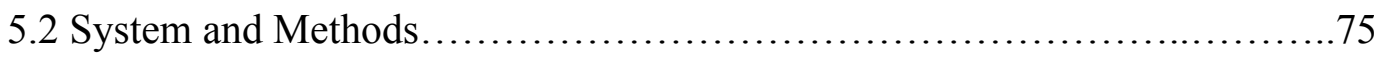

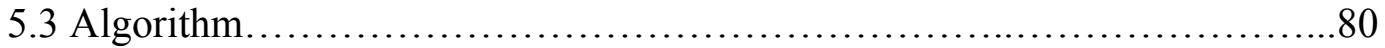

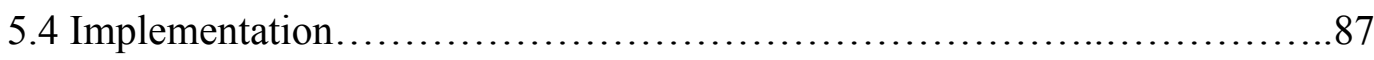

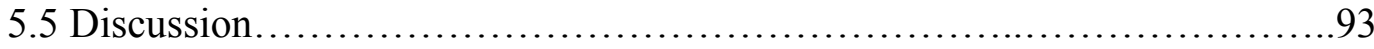

6. Inference of Cohort-Specific HIV-1 Kinetics................................... 97

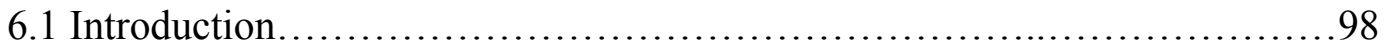

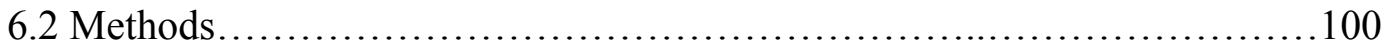

6.2.1 Selection of Subject Data..................................... 100

6.2.2 Optimization of Models Using an Evolutionary Algorithm.........101 


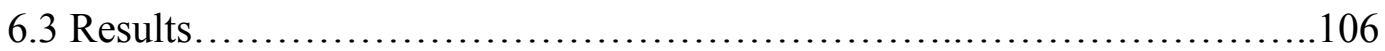

6.4 Discussion......................................................... 110

7. Conclusions and Future Directions............................................. 112

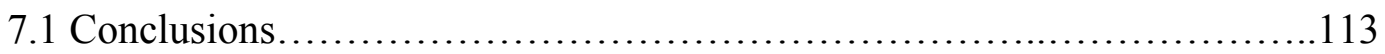

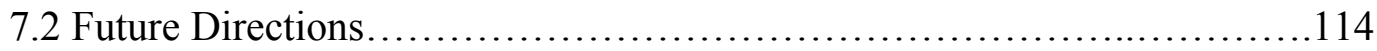

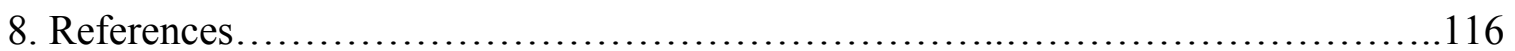

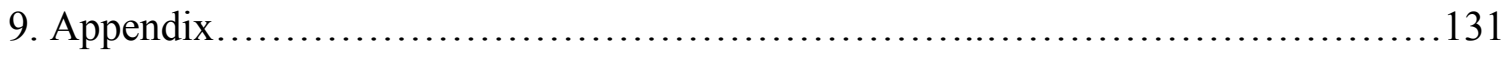

9.1 Appendix 1: Elite Variant Selection Strategy Code.......................132

9.2 Appendix 2: In silico HIV-1 Positive Subject Population Analysis Code....133

9.3 Appendix 3: Biochemical Kinetics Inference Algorithm Code..............137

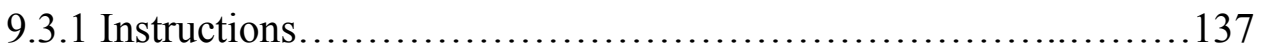

9.3.2 EvolutaionaryAlgorithm_SingleVariableStep.nb..................140

9.3.3 EvolutaionaryAlgorithm_SingleVariableStep

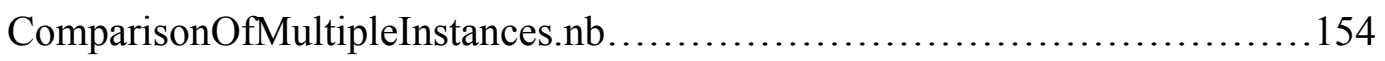

9.3.4 EvolutaionaryAlgorithm_SingleVariableStepComparison

OfMultipleInstancesWorkAround.nb...................................... 164

9.3.5 EvolutaionaryAlgorithm_CompleteModel

Optimization(FinalStep) ..................................173 


\section{Table of Figures}

Figure 2.1: Oral Mucosal Biopsies In Autologous Stem Cell Transplant Patient.........10

Figure 2.2: Oral Mucositis Network Schematic................................. 15

Figure 2.3: Results of Oral Mucositis Simulations...............................18

Figure 3.1: Genetic Algorithm Using Elite Variant Selection...........................26

Figure 3.2: HIV Dynamic Model................................................. 31

Figure 3.3: Evolution of Solutions in Function Tests................................ 35

Figure 3.4: Evolution of Satisfiability Test Solutions............................39

Figure 3.5: Evolution of Highly Active Antiretroviral Therapy (HAART) for Treatment of Human Immunodeficiency Virus (HIV) .......................4

Figure 3.6: Optimized Highly Active Antiretroviral Therapy (HAART) Regimens......42

Figure 3.7: Effect of Optimized HAART on HIV Model Variables....................44

Figure 4.1: HIV Dynamic Model..............................................53

Figure 4.2: HAART Treatment Strategy Optimized for the Average Subject............59

Figure 4.3: HAART Range of Coverage for in silico Cohort Using Standard and

Customized Treatment...........................................61

Figure 4.4: Comparison of Clinical Range Of Coverage (ROC) to in silico ROC.......662

Figure 5.1: Parse-tree Solution Structure for Genetic Programming ...................73

Figure 5.2: General Viral Dynamics System.................................... 76

Figure 5.3: Mapping of Model Genotype to Model Phenotype in Evolutionary

Algorithm.

Figure 5.4: Algorithm for the Discovery of a Biochemical Kinetic Model Describing a 3-

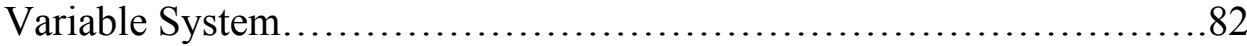


Figure 5.5: Evolutionary Algorithm for the Optimization of Biochemical Model

Equations................................................ 84

Figure 5.6: Evolution of the General Viral Dynamics Model..........................89

Figure 5.7: Simulation of Evolved Viral Dynamics Models........................91

Figure 6.1: $\left[\mathrm{CD}^{+}\right]$and [HIV-1] Data Describing HIV-1 Positive Subject Cohorts......101

Figure 6.2: An Evolutionary Algorithmic Approach for the Optimization of Cohort-

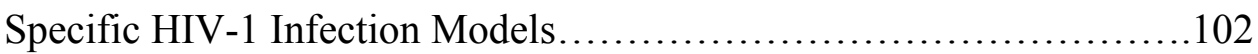

Figure 6.3: Representation of Potential Models in Evolutionary Algorithmic

Approach...................................................104

Figure 6.4: Evolved Cohort-Specific Reaction Kinetic Models......................107

Figure 6.5: Cohort Model Simulations and Comparison to Clinical Data................109 


\section{Table of Tables}

Table 2.1: Oral Mucositis Model Parameters......................................17

Table 3.1: Genetic Algorithm Function Tests.....................................29

Table 3.2: HIV Dynamic Model Parameters.................................... 33

Table 4.1: Total HAART Dosage Prescribed By Each Treatment Regimen..............52

Table 4.2: HIV Dynamic Model Parameters.......................................54

Table 4.3: Average Subject's Initial Conditions, Pre-Treatment......................56

Table 4.4: Initial Conditions at the Initiation of Treatment Optimization..............56

Table 5.1: Viral Dynamics Model Parameters................................... 77

Table 5.2: Viral Dynamics Model Initial Conditions For Data Generation................88

Table 5.3: Viral Dynamics Model Test Initial Condition..........................92

Table 5.4: Comparison of Known Equations to Generated Equations...................93

Table 6.1: Cohort-Specific Model Reaction Parameters............................. 108 
CHAPTER 1

INTRODUCTION 


\subsection{Motivation}

Mathematical models are used in nearly all industries to analyze data, make predictions, and to propose improvements based upon those predictions. Most models describing data sets are defined by optimizing parameters taken from pre-existing model structures. Genetic programming allows one to carry out a symbolic regression to simultaneously fit both the equation structure and the parameters to a data set requiring only an input of the model variables and a set of available mathematical functions $\{+,-$, *, /, etc (Holland 1975, Koza 1992). Thus far, model generation by genetic programming has been restricted to the evolution of simple models due to challenges in navigating the size of the search space for complex systems of equations.

Recently, work has been done on model generation from experimental data sets without assuming any prior knowledge about system mechanism. Schmidt et al.'s Eureqa formulize, a symbolic regression package that uses a genetic programming approach, quickly fits models to input data (Schmidt and Lipson 2009). Although these models provide a good fit to experimental data, they often do not provide mechanistic information as to how species in a biological system interact. Chattopadhyay et al. developed an algorithm for inferring stochastic reaction mechanism from experimental data (Chattopadhyay et al. 2013). This algorithm was successful at generating correct mechanistic models on the algorithm's error-complexity Pareto front, but some knowledge of the system was required in order to select the correct model from a set of potential models. A study by Bazil et al. provided a method for the reverse engineering of biological networks by generating systems of ordinary differential equations from experimental data (Bazil et al. 2011). This algorithm was successful in identifying 
candidate pathways that were known to exist but also generated many false positive pathways, as the algorithm was designed to minimize the number of false negative pathways identified.

A common method used in these approaches is that of genetic programming. Genetic programming is a type of evolutionary algorithm that generates programs or models of varying size and structure while not assuming any pre-defined model structure. The model is chosen by defining a set of functions (i.e. $+,-,{ }^{*}, /$ ), and a set of terminals (i.e. variables, real numbers) as building blocks from which to construct equations (Koza 1992). Genetic programming works by generating a population of potential models of a problem where each solution consists of the defined functions and terminals. The potential models are ranked or scored based upon a defined fitness function. Models are then selected, usually in proportion to their fitness score, to undergo recombination with other models as well as for mutation. New models are incorporated with selected existing models and the new population of models is carried on to the next iteration where the algorithm repeats itself. A few examples where genetic programming has been used to create models addressing many different problems include the evolution of natural laws (Iba 2008, Schmidt and Lipson 2009), computer vulnerability testing (Kayacik et al. 2011), prediction of longitudinal dispersion coefficients in streams (Azamathulla and Ghani 2011), protein binding sites (Bains et al. 2004), interpretation of microbial flow cytometric data (Davey and Davey 2011), embedding and decoding of digital watermarks (Usman et al. 2011), synthesis of polymorphic combinational circuits (Gajda and Sekanina 2011), pipe break prediction modeling (Xu et al. 2011), estimation of daily pan evaporation (Shiri and Kisi 2011), software engineering predictive modeling (Afzal and 
Torkar 2011), modular neural network programming (Tsai and Lin 2011), selfreproducing machines (Zykov et al. 2005), and steel beam load capacity prediction models (Gandomi et al. 2011).

A desirable enhancement to the genetic programming approach would lead to an extension of its use to the efficient generation of more complex mathematical models. These models, such as multi-variate systems of ordinary differential equations, would expand the use of this approach to model multi-variate data sets as are commonly found in biological systems. Gene expression programming (GEP) has been proposed as an enhancement to genetic programming (Ferreira 2006). In a GEP-type approach, solutions are encoded as a linear sequence of functions and terminals during the selection, recombination, and mutation operations. The encoded solutions are then mapped to equations for simulation and scoring.

Mathematical modeling of viral systems such as HIV-1, hepatitis B, and others has been of great interest in the hopes of developing treatment strategies based upon those models (de Sousa and Cunha 2010, Guedj and Neumann 2010, Krishnan 2011, Srivastava et al. 2002). Many models of various sizes and structures have been proposed for these systems taking into account a wide range of biochemical species. Depending on the species taken into account and their predicted relationships, the model behavior can vary in response to perturbation. The most comprehensive approach to determine optimal models describing such biochemical systems would be to assume no prior knowledge about the systems and the way that the species interact. A symbolic regression approach such as a GEP-based evolutionary algorithm could then be utilized to derive the mechanistic model describing the species, taking into account all necessary species 
interactions including some that may not be inherently obvious. The resulting model could then be used to make predictions about the system as well as to test and optimize potential therapeutics.

Human immunodeficiency virus type 1 (HIV-1) is an important disease that has received significant interest from the modeling community (Aviran et al. 2010, Bonhoeffer et al. 1997(a,b), Burg et al. 2009, Herz et al. 1996, Nowak et al. 2000, Perelson et al. 1996, Perelson 2002, Prosperi et al. 2009, Ribeiro and Bonhoeffer 2000, von Kleist et al. 2010, Wu and Zhang 2010, Zeng and Yang 2010). In recent years, HIV1 treatments have significantly reduced AIDS-related mortalities and have enhanced long-term disease control (Dybul et al. 2001, Fagard et al. 2003, Powderly 2002, Ruiz et al. 2001). These benefits have been realized in large part via use of highly active antiretroviral therapy (HAART) in clinical practice. The HAART strategy consists of treating the disease with a combination of antiretroviral drugs. Despite advances in efficacy over prior generation treatments, HAART is associated with risk of serious side effects that include cardiovascular disease (Domingos et al. 2009), nephrotoxic effects (Izzedine et al. 2009), and oral toxicities (Nittayananta et al. 2010). Up to $16 \%$ of patients require modification or discontinuation of their HAART regimen due to drugrelated toxicities (El-Sadr et al. 2006).

\subsection{Specific Aims}

I hypothesize that using reaction kinetic theory to constrain model space, it is possible to infer mechanistic mathematical models of complex biological interactions by 
using an evolutionary algorithm to carry out symbolic regression. To test this hypothesis, the following specific aims were pursued:

1. The development of a GEP-based evolutionary algorithm that optimized reaction kinetic models of biological systems from time series data sets.

2. The generation of cohort-specific reaction kinetic models of Human Immunodeficiency Virus (HIV) infection from a clinical data set.

To address these specific aims, the current symbolic regression methodology termed genetic programming was enhanced using a GEP-based approach to evolve more complex mechanistic reaction kinetic models describing multi-variate data sets. Also, experience in modeling HIV infection and simulating treatment outcomes was used to identify a clinical data set on which the GEP-based evolutionary algorithm was applied.

Completion of aims 1 and 2 resulted in an algorithm that could efficiently determine a model structure and parameters to a moderately sized time series data set. The algorithm required no prior knowledge of the relationship among measured variables. The enhancements to the genetic programming approach also allowed for the generation of models containing many variables. Interactions between large numbers of variables are commonly found in biological systems. The algorithm therefore will be beneficial in generation models of complex biological systems. Models describing biological systems have the potential to guide the discovery of drug targets and therapeutic strategies. This technology also has the potential to be useful in personalized medicine applications. The tailoring of mathematical models to an individual's disease state based upon their unique biochemical kinetic markers would allow clinicians to make 
predictions about an individual's disease state and to optimize treatment to the individual patient.

\subsection{Outline}

In Chapter 2, it is demonstrated how mathematical modeling can be used to simulate the progression of a disease. A mass action kinetic model was used to describe nine biochemical species involved in the pathogenesis of oral cavity mucositis. The parameters of the model were optimized to a heterogeneous data set based on the shared pathway assumption.

In Chapter 3, the topic of evolutionary algorithms is introduced as the performance of the genetic algorithm operation termed "selection" was evaluated. It was found that a novel selection strategy, termed elite-variant selection, greatly improved the performance of the genetic algorithm in a variety of application tests. One of these tests included the optimization of a theoretical Highly Active Antiretroviral Therapy (HAART) regimen to an in silico HIV-positive subject.

In Chapter 4, a systems biology analysis is applied to clinical HAART regimens, termed Standard Treatment Interruption (STI) regimens, which were utilized to potentially treat a subject's HIV while also administering less HAART. Clinically, these STI regimens were found to be unsuccessful compared to standard HAART and the systems biology approach was able to predict this without exposing subjects to a clinical trial. Further, using a systems biology approach it was shown that an optimal HAART regimen could be found that would control a subject's HIV while also minimizing HAART dose and thereby reducing the risk of serious side effects. 
In Chapter 5, an evolutionary algorithm is described that evolves reaction kinetic models of biochemical systems from time series data sets. This was accomplished by enhancing the genetic programming methodology by using a GEP-based approach where reaction kinetic knowledge is leveraged and potential models are considered and optimized. This algorithm, significantly, assumes no prior knowledge of how biochemical species that play key roles in disease pathogenesis interact and it allows for the discovery of pathways that may exist between these species.

In Chapter 6, the GEP-based evolutionary algorithm was used to evolve cohortspecific reaction kinetic models describing clinical data taken from HIV-positive subjects. These models described important pathways that were active in the different cohorts and were able to give insight as to the effectiveness of HAART regimens in controlling the propagation of HIV in these cohorts.

This work is concluded in Chapter 7 and Chapter 9 contains the Appendices. In Appendix 1, Common Lisp code of the elite-variant selection strategy is provided to complement Chapter 3. In Appendix 2, Common Lisp code that generates in silico HIVpositive populations and simulates HAART on these populations is provided to complement Chapter 4. In Appendix 3, the GEP-based evolutionary algorithm and associated packages are provided as Mathematica code. This code complements Chapter 5 and was applied to HIV-positive cohort data in Chapter 6. 
CHAPTER 2

MODELING OF ORAL CAVITY MUCOSITIS 


\subsection{Introduction}

One example of the utility of using mathematical models to describe biological phenomena is the optimization and analysis of a network describing oral cavity mucositis. The work presented in this Chapter is my contribution of the work published in the proceedings of the American Society of Clinical Oncology (Peterson et al. 2008, Srivastava and White et al. 2011). Oral cavity mucositis is a condition observed in cancer patients receiving radiation therapy or chemotherapy. It is characterized by inflammation followed by sepsis and bacterial infection in the oral cavity (Knox et al. 2000, Niscola et al. 2007, Peterson 1999, Sonis 2004). The result of this condition is significant pain, interruption of therapy, increased costs due to hospitalization, and in some cases death (Lalla et al. 2008, Peterson et al. 2007). Figure 2.1 depicts changes in the oral mucosa during as a result of this disease.

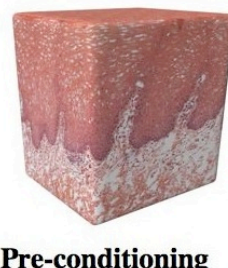

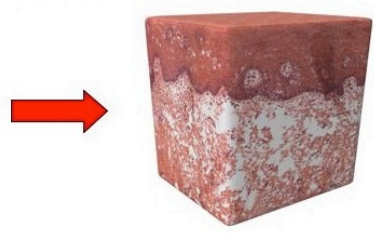

Day +10

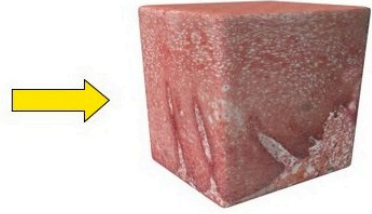

Day +28

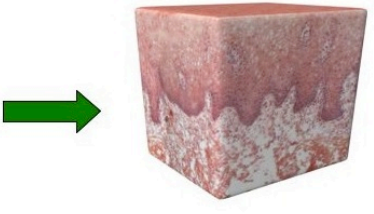

Day +100

Figure 2.1. Oral Mucosal Biopsies In Autologous Stem Cell Transplant Patients (Peterson et al. 2008). The histopathologic specimens shown here were directly obtained via oral mucosal biopsy specimens in autologous stem cell transplant patients prior, during and following resolution of acute mucositis caused by a high-dose conditioning regimen. The specimens illustrate tissue-based changes that are relevant to oral mucositis data. 
Recent basic research advances have strategically contributed to (i) defining pathobiology of alimentary tract mucosal injury secondary to cancer therapy and (ii) linking molecular mechanisms with clinically important outcomes. Use of analytic technologies to integrate biological, clinical and computational domains represents an important new direction in the field, such that gaps in existing knowledge can be translated into novel hypotheses as well as prediction of experimental outcomes. The prototype presented in this study provides the basis for development of a detailed mathematical model for quantifying relevant components of the mucositis pathway.

Oral and gastrointestinal mucositis can be a major toxicity secondary to high-dose cancer therapies, including chemotherapy and radiation administered as single agent or combined treatment (Peterson and Sonis 2001). Potential clinical and mechanistic relationships between selected targeted cancer therapies and mucosal injury have recently been reported as well (Keefe and Gibson 2007). Nearly all patients receiving radiation therapy for head and neck cancer develop clinically significant oral mucositis, with $16 \%$ requiring hospitalization because of its severity (Lalla et al. 2008). Severe oral mucositis results in unplanned radiation therapy treatment interruptions in $11 \%$ of patients. Patients undergoing multi-cycle chemotherapy for solid tumors are also at risk. For example, 303 of 599 patients $(51 \%)$ receiving chemotherapy for solid tumors or lymphoma developed at least World Health Organization Grade 3 oral and/or gastrointestinal mucositis. Reduction in subsequent dose of chemotherapy was twice as common after cycles with mucositis than after cycles without mucositis, thus creating the potential for less than optimal tumor response. 
Current work is fostering development of novel, molecularly targeted interventions to reduce severity and duration of oral mucositis in selected cancer patient cohorts (Peterson et al. 2007). Important principles of the current pathobiologic model of mucositis include the role of pro-inflammatory cytokines (Sonis 2007), tissue-based genetic risk and resistance mechanisms (Belinsky et al. 2007, Boerma et al. 2007, Duan et al. 2007) and symptom clusters (Aprile et al. 2008, Campagnaro et al. 2008). However, key gaps in knowledge remain across the molecular, tissue and clinical continuum relative to strategically advancing this multidimensional modeling. In this study, it was shown that Virtual Cell (http://vcell.org) (Schaff et al. 1997, Slepchenko et al. 2003) represents a unique technology to address these complex research issues. Effort was directed to development of a new computational strategy to bridge "top down", clinically-based events with complex, "bottom up" molecular pathways. It was demonstrated that using computational modeling fosters integration of data from recent studies (Jain et al. 2006, Knorr and Srivastava 2005, Moraru and Loew 2003, Schaff et al. 1997, Slepchenko et al. 2003) that have characterized pathways associated with up-

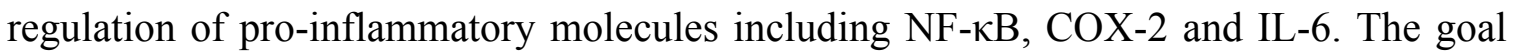
of this research was to provide new molecular insights into both causation of alimentary tract mucosal injury in cancer patients as well as the relationship of associated pathways to clinical consequence with the hope of forming novel hypotheses on how to downregulate this condition. 


\subsection{Methods}

Integration of available data into a coherent model is an important approach to precisely defining the collective molecular complexities of mucositis. Determination of the physiological characteristics that the model is able to capture is designed to elucidate specific components and mechanisms. The current paradigm capitalizes on prior studies in order to generate this new line of research (Jain et al. 2006, Knorr and Srivastava 2005, Moraru and Loew 2003, Schaff et al. 1997, Slepchenko et al. 2003). The current view of mucositis pathogenesis includes the theory of multiple shared pathways across the continuum of alimentary tract mucosa (Sonis 2007). A "systems biology" approach, in which data collected from these shared pathways are leveraged and integrated into a comprehensive computational model of mucositis, can potentially define commonalities and differences among causation of the collective mucosal injury. Using these shared pathways as a foundation, it is possible to apply the theory of reaction kinetics to develop a mathematical model of the interactions among what are believed to be key components involved in mucositis. The current model that I assembled accounts for interaction dynamics among COX-1, COX-2, TNF- $\alpha$, PGE-2, IL-1 $\beta$, IL-6, PGI-2, TXA-2 and NF$\kappa \mathrm{B}$, and includes the effect of biological feedback loops among all the species (Peterson et al. 2008). The model is described by Equations 2.1-2.9 and is depicted as a network structure in Figure 2.2. 


$$
\begin{gathered}
\frac{d[N F-\kappa B]}{d t}=k_{1}+k_{2}[T N F-\alpha]+k_{3}[I L-1 \beta]+k_{4}[P G E-2] \\
-k_{5}[N F-\kappa B] \\
\frac{d[T N F-\alpha]}{d t}=k_{6}+k_{7}[N F-\kappa B]-k_{8}[T N F-\alpha] \\
\frac{d[I L-1 \beta]}{d t}=k_{9}+k_{10}[N F-\kappa B]-k_{8}[I L-1 \beta] \\
\frac{d[C O X-2]}{d t}=k_{12}-k_{13}[C O X-2] \\
\frac{d[C O X-1]}{d t}=k_{14}-k_{15}[C O X-1] \\
\frac{d[P G E-2]}{d t}=k_{16}[C O X-2]+k_{17}[C O X-1]-k_{18}[P G E-2] \\
\frac{d[P G I-2]}{d t}=k_{19}[C O X-2]+k_{20}[C O X-1]-k_{21}[P G I-2] \\
\frac{d[T X A-2]}{d t}=k_{22}[C O X-2]+k_{23}[C O X-1]-k_{24}[T X A-2] \\
\frac{d[I L-6]}{d t}=k_{25}+k_{26}[N F-\kappa B]-k_{27}[I L-6]
\end{gathered}
$$




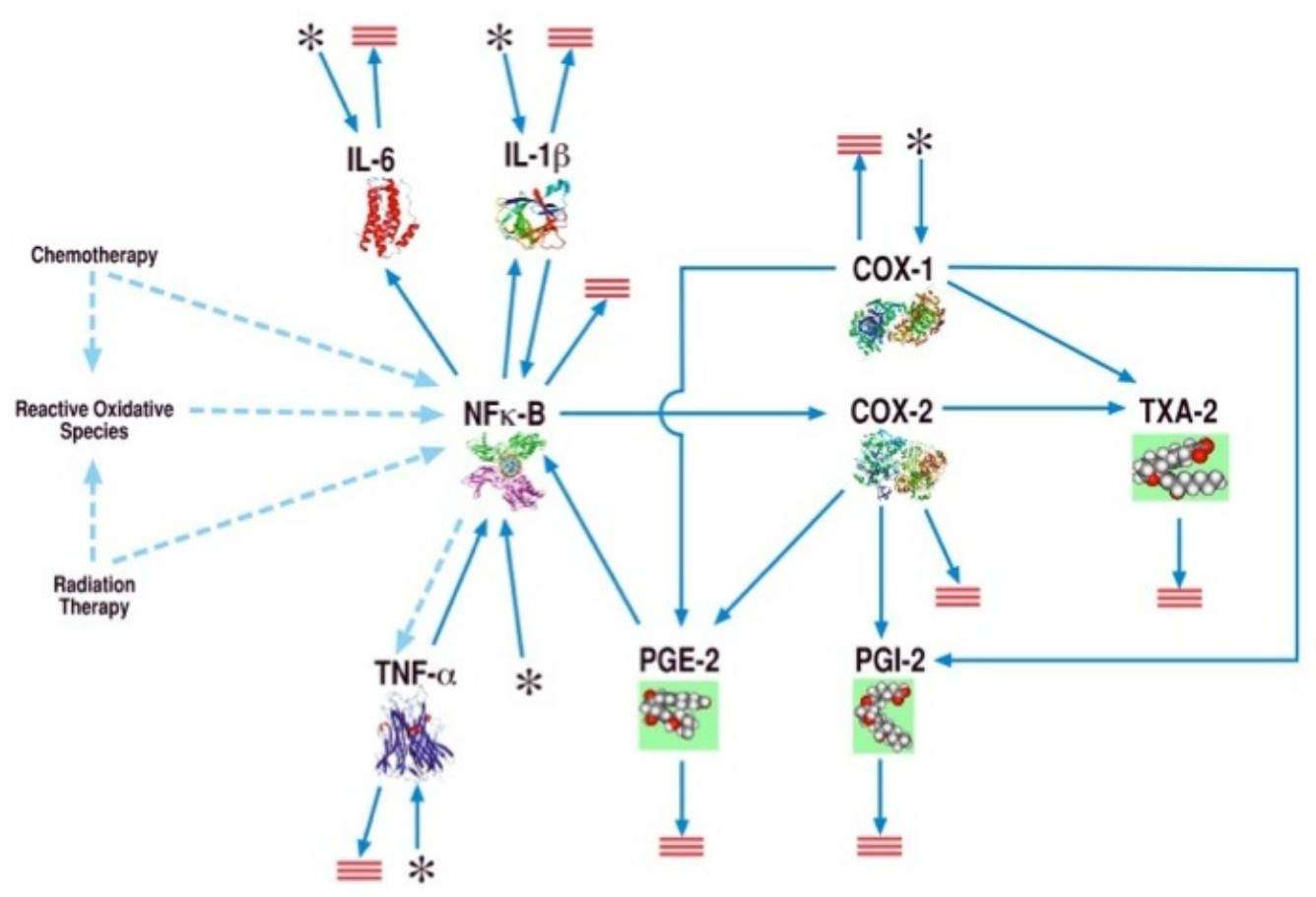

Figure 2.2. Oral Mucositis Network Schematic (Peterson et al. 2008). Oral mucositis is initiated by chemotherapy or radiation therapy. The transcription factor NF- $\mathrm{BB}$ is activated directly by these therapies or through the production of reactive oxidative species. NF- $\mathrm{B}$ upregulates many genes including the cytokines IL-6, IL- $1 \beta$, and TNF- $\alpha$. The enzymes COX-1 and COX-2 are also up-regulated which in turn catalyze the production of the PGE-2, PGI-2, and TXA-2 cytokines. PGE-2, IL-1 $\beta$, and TNF- $\alpha$ can feedback to activate additional NF- $\kappa$ B. The “*” represents constitutive processes and red bars denote degradation reactions.

To conduct the computational simulation, it was necessary to associate a parameter with each reaction. Estimation of parameter values was conducted using the Virtual Cell software platform (Schaff et al. 1997, Slepchenko et al. 2003), based on a combination of data in the literature and primary experimental data. Ideally, the collective kinetic data should be based on a single cohort of human studies of oral mucositis. However, the relatively limited scope of published data often dictates that heterogeneous 
data sources be used. These sources may include data from different human studies, or from studies using animal models such as murine or rat. A literature survey, conducted to delineate necessary data to estimate the parameters, determined that the quantity of kinetic data for many of the oral mucositis reactions was sparse to non-existent, even when evaluating heterogeneous data sources. For example, no data on PGE-2 kinetics for oral mucositis was found in any animal model or human studies. Based on the shared pathway assumption, PGE-2 kinetic data from murine inflammatory bowel disease (IBD) modeling was ultimately utilized (Melgar et al. 2006).

\subsection{Results}

Reaction parameters for the oral mucositis model were estimated using Virtual Cell and are reported in Table 2.1. Once parameters for the model based on data from oral mucositis and IBD were estimated, it was possible to carry out simulations of the mucositis process. The timeframe examined was divided into two components. The first 15 days post-chemotherapy involved the "injury and wounding phase" of tissue toxicity. The remaining 85 days were identified as the "healing phase." It was experimentally observed that seven of the nine species reached a plateau in species concentration over the observed 100-day timeframe (Peterson et al. 2008). The only species not to reach a plateau during the healing phase were TXA-2 and PGI-2. The model was capable of predicting the difference between the initial and plateau values of the seven remaining species within experimental error. 


\begin{tabular}{|c|c|c|c|c|c|}
\hline $\mathrm{k}_{1}$ & 12.49 uM NF-kB/day & $\mathrm{k}_{10}$ & $\begin{array}{l}\text { 100.97 mRNA/GAPDH/(day*uM } \\
\text { NF-kB) }\end{array}$ & $\mathrm{k}_{19}$ & $51.63 \mathrm{pg} / \mathrm{ml} /($ day*ng mRNA $)$ \\
\hline $\mathrm{k}_{2}$ & 10.10 uM NF-kB/(day*mRNA) & $\mathrm{k}_{11}$ & $90.671 /$ day & $\mathrm{k}_{20}$ & $448.04 \mathrm{pg} / \mathrm{ml} /\left(\right.$ day*ng mRNA) $^{*}$ \\
\hline $\mathrm{k}_{3}$ & $12.21 \mathrm{uM} \mathrm{NF- \kappa B/(day*mRNA)}$ & $\mathrm{k}_{12}$ & 119.97 ng RNA/(day*uM NF-kB) & $\mathrm{k}_{21}$ & $0.021 /$ day \\
\hline $\mathrm{k}_{4}$ & $\begin{array}{l}2.71 * 10^{-6} \text { uM NF-kB/(day*pg/100 } \\
\mathrm{mg} \text { tissue) }\end{array}$ & $\mathrm{k}_{13}$ & $2.711 /$ day & $\mathrm{k}_{22}$ & $55.59 \mathrm{pg} / \mathrm{ml} /($ day*ng mRNA $)$ \\
\hline $\mathrm{k}_{5}$ & $53.171 /$ day & $\mathrm{k}_{14}$ & 1738.18 ng mRNA/day & $\mathrm{k}_{23}$ & $3675.93 \mathrm{pg} / \mathrm{ml} /($ day*ng mRNA) \\
\hline $\mathrm{k}_{6}$ & $463.67 \mathrm{mRNA} / \mathrm{GAPDH} / \mathrm{day}$ & $\mathrm{k}_{15}$ & $6.12 * 10^{-18} 1 /$ day & $\mathrm{k}_{24}$ & $0.031 /$ day \\
\hline $\mathrm{k}_{7}$ & $\begin{array}{l}5.28 * 10^{-19} \\
\text { mRNA/GAPDH/(day*uM NF- } \kappa B)\end{array}$ & $\mathrm{k}_{16}$ & $\begin{array}{l}8.88 * 10^{-19} \mathrm{pg} / 100 \mathrm{mg} \\
\text { tissue/(day*ng mRNA) }\end{array}$ & $\mathrm{k}_{25}$ & $36.36 \mathrm{mRNA} / \mathrm{GAPDH} / \mathrm{day}$ \\
\hline $\mathrm{k}_{8}$ & $1269.851 /$ day & $\mathrm{k}_{17}$ & $\begin{array}{l}40.54 \mathrm{pg} / 100 \mathrm{mg} \text { tissue/(day*ng } \\
\text { mRNA) }\end{array}$ & $\mathrm{k}_{26}$ & $\begin{array}{l}56.80 \mathrm{mRNA} / \mathrm{GAPDH} /(\text { day*uM } \\
\text { NF- } \mathrm{\kappa B})\end{array}$ \\
\hline $\mathrm{k}_{9}$ & 105.16 mRNA/GAPDH/day & $\mathrm{k}_{18}$ & $0.041 /$ day & $\mathrm{k}_{27}$ & $351.371 /$ day \\
\hline
\end{tabular}

Results for five of the seven species are shown in Figure 2.3, with the remaining two species not shown due to the differences in scale. In studying the dynamics during the injury and wounding phase of the molecular species involved in mucositis, the model was able to reproduce the dynamics of TXA-2 and PGI- 2, which were the only species not to reach a plateau (Peterson et al. 2008). The model was unable to reproduce the dynamics observed in this phase for the other species. The inability to reproduce these dynamics may be due to a number of reasons: (i) the interactions among the components may be incomplete or erroneous; (ii) the differences among kinetics of the shared pathways may be sufficiently significant to make the shared pathway assumption a poor one in this case; or (iii) variation among different data sources (human, mouse, and rat) may be result in substantial, detrimental impact estimation of reaction parameters. This work therefore highlights the importance of conducting appropriately designed experiments from a single cohort of test subjects. In doing so, it will be possible to remove inaccuracies associated with the last two confounders cited above, namely, the shared pathway assumption and the use of heterogeneous datasets. 


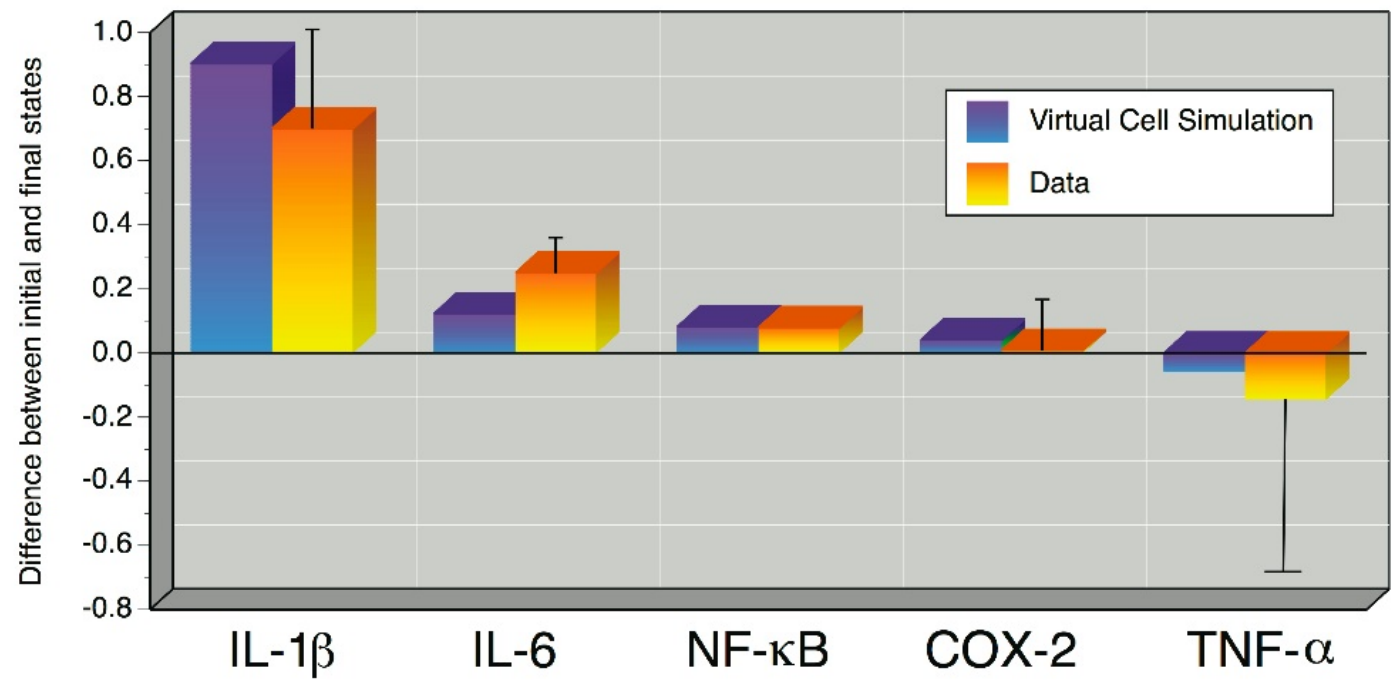

Figure 2.3. Results of Oral Mucositis Model Simulations (Peterson et al. 2008). The difference between initial species level and final plateau levels are illustrated for the Virtual Cell simulation versus experimental data and data from the literature (Lipniacki et al. 2004, Sun et al. 2001). The units for NF- $\kappa B$ are $\mu \mathrm{m}$, for COX-2 are ng of mRNA, and for IL- $1 \beta$, IL-6 and TNF- $\alpha$ are mRNA levels normalized for GAPDH. Note that the NF- $\mathrm{B}$ data derive from an independent simulation (Lipniacki et al. 2004); they thus do not have an associated experimental error. This analytic strategy with NF- $\mathrm{kB}$ illustrates the ability of the modeling to integrate data from diverse sources.

\subsection{Discussion}

Mucositis can be a significant dose-limiting complication of cancer therapy. Higher-dose cancer treatment regimens might become feasible if the toxicity could be prevented or substantially reduced in severity. More aggressive cancer therapies could in turn produce higher cancer cure rates and/or more durable remissions. There are a number of potential benefits to utilizing a systems biology approach to enhance the modeling of mucositis. The simulations completed to date have been important in two 
respects. First, they have highlighted key gaps in the literature that need to be addressed. Second, a number of key experiments have been suggested, along with how those experiments should be designed. An example of implementing these two outcomes is directed to detailed analysis of interactions among species during the injury and wounding phase, particularly at the very early stages. To this end, an important specific experiment would involve study of kinetic data for PGE-2 dynamics. Furthermore, a homogeneous dataset needs to be collected from a single cohort for the molecular species in question. Ideally, a human cohort should be utilized. However, studies from animal models could also prove to be highly beneficial if integrated into a single, comprehensive experiment. As the model matures, it will permit study of additional key avenues of research including studying the mechanisms by which selected oral mucosal sites (e.g., hard palate) are relatively resistant to high-dose cancer therapy, while other sites are highly susceptible to injury (e.g., buccal mucosa, soft palate) and selected non-oral mucosal sites (e.g., conjunctival mucosa) are rarely clinically affected by high-dose chemotherapy, while other sites (e.g., selected oral mucosal sites) are highly susceptible.

Future applications could also include the study of pharmacologic interventions that enhance tumor susceptibility to cancer therapy as well as reducing mucosal injury. This technology represents an innovative strategy to accelerate future laboratory and clinical investigations directed to network pathways and cancer treatment research. The model-based analysis has potential for utilization for personalized medicine. By determining the specific parameters of an individual, the technology could evaluate customized mucositis management strategies for that patient, with resultant reduction in cancer treatment toxicity. 
CHAPTER 3

DEVELOPMENT OF AN ELITE-VARIANT SELECTION STRATEGY FOR GENETIC ALGORITHM OPTIMIZATION 


\subsection{Introduction}

The work presented in this Chapter is my contribution towards an oral presentation given by me at the Biomedical Engineering Societies Annual Meeting in 2011 (White and Srivastava 2011). Significantly, I demonstrate how an improvement to the genetic algorithm selection operation results in rapid convergence of the algorithm in many cases. Because genetic algorithm approaches are often computationally intensive, this approach has the benefit of greatly reducing the time needed to converge upon a desired solution.

Genetic algorithms are a subset of evolutionary algorithms used to optimize solutions to complex problems by means of evolving a solution most fit to solve a given problem from a pool of possible solutions (Arnold and Beyer 2006, Bäck 1996, Fang et al. 2010, Goldberg 1989, Holland 1975, Iba 2008, Schwefel 1993, Tang and Wu 2009). During the course of this evolution, possible solutions, referred to as chromosomes, are selected to undergo recombination and mutation so that better solutions may be discovered while also maintaining diversity in the population of solutions. This diversity in the population of solutions allows for a broad search of the solution space so that the population will not prematurely converge to a local optimum. The cycle of selection, recombination, and mutation either repeats itself for a set number of iterations, also known as generations, or until a specified termination criterion has been reached. Although extensive research on different approaches for carrying out selection, recombination, and mutation in evolutionary algorithms has been carried out (De Jong and William 1991, Deb et al. 2002, Eremeev 2008, Gallagher and Frean 2005, Gero and Kazakov 2001, Jansen et al. 2005, Nomura and Shimohara 2001, Rowe et al. 2004, 
Whitley 1989), this work will focus primarily on selection.

Common examples of selection methods include Tournament selection, Proportional selection, Linear Ranking selection, and Elite selection. The Tournament selection approach involves selecting the chromosome which is the best fit of a randomly populated sub-population of specified size to take part in recombination and mutation with another chromosome selected in the same manner (Blickle and Lothar 1995). By doing this, Tournament selection balances convergence of the population to an optimum with maintaining population diversity so that the population does not become uniform at a local optimum thereby giving it a chance to arrive at the global optimum. Likewise, Proportional selection maintains this balance by assigning a weight to each chromosome proportional to its fitness score (Schwefel 1993). In this way, selecting a chromosome to take part in recombination and mutation can be related to spinning a roulette wheel where the slot size is proportional to the fitness of the chromosome with the most fit having the largest slot and the least fit having the smallest slot. Like Tournament selection, the fittest chromosome in a population is not guaranteed to enter into recombination or be carried to the next generation, but has the greatest likelihood of doing so.

Linear Ranking selection attempts to overcome the bias that may enter Tournament or Proportional selection when a "super chromosome", or a chromosome that is significantly fitter than other chromosomes, enters the population by weighting chromosomes in the population relative to fitness rank rather than fitness score (Baker 1985). A linear relationship between rank and likelihood of being selected is then established so that the top ranked chromosome will be the most likely to be selected, and the last ranked chromosome will be the least likely to be selected. Addressing this bias in 
Proportional and Tournament selection may avoid a situation where a "super chromosome" is continuously selected for recombination, leading to a homogenized population that plateaus at a local optimum resulting in an undesirable solution.

Finally, Elite selection is a method that balances convergence with population diversity by copying an "elite" fraction of chromosomes untouched to the next generation, thereby keeping better scoring genetic material for use in the next generation (Costa and Oliveira 2003). The remainder of the new generation is populated by chromosomes that are randomly selected and are either simply copied or undergo recombination and/or mutation based on some pre-determined probability. This selection method has the advantage of being able to progress towards the optimum by means of keeping the best genetic material in the population. Elite selection also maintains population diversity by selecting random chromosomes for recombination and mutation without regard to fitness score or rank.

The goal of this work was to achieve an improvement in the balance between convergence and population diversity in genetic algorithm selection, which will ultimately allow desirable solutions to be reached faster. It is hypothesized that the selection process in genetic algorithms can be configured in such a way that convergence to a global optimum or termination criterion will occur in a timely fashion for a large scope of problems, while also broadly probing the search space in order that the population of solutions does not plateau at a local optimum. Such a selection method would offer a clear improvement over currently existing selection methods. In an effort to address these goals, an Elite Variant (EV) selection strategy is presented. The EV selection strategy has been designed to take the desirable aspects of Elite selection one 
step further to a selection strategy that converges more rapidly than the Elite selection method while maintaining the ability to preserve a diverse population and a broad search of the solution space.

\subsection{Methods}

\subsubsection{Development of Elite Variant Selection Genetic Algorithm}

Genetic algorithms follow a common methodology of utilizing selection, recombination, and mutation operators to evolve a population of possible solutions towards the optimal solution. The selection operator determines which possible solutions or chromosomes are selected to undergo recombination. The recombination operator then determines whether the selected chromosomes will pass to the next generation unaltered or recombined. If the chromosomes are to be recombined, one or more chromosome positions are randomly selected at which genetic material from the selected chromosomes will be exchanged. Finally, each position or allele in each selected chromosome is given a small chance to be mutated to a different value.

Implementation of a genetic algorithm using Elite Variant (EV) selection, summarized in Figure 3.1, may be carried out as follows. An initial pool of a specified size is populated by possible solutions or chromosomes. The user specifies the nature of these possible solutions. The chromosomes in the population are then scored in relation to a defined scoring metric. Depending on the nature of the problem, one may wish to maximize or minimize the scores of the chromosomes to a desired global optimum or user-specified termination criterion. For the first iteration or generation, the algorithm behaves as the Elite selection strategy algorithm does, and a fixed percentage of the best 
scoring chromosomes are copied to the next-generation population. For this study the top $20 \%$ of the best scoring chromosomes was chosen to be copied to the next generation when using the Elite selection strategy, although another appropriate fraction could be chosen.

The final $80 \%$ of the next generation are then composed of pairs of chromosomes, which are selected randomly and given a $70 \%$ probability to breed or recombine with each other. After this recombination step, each part of both chromosomes is given an opportunity to mutate. The chance of mutation is set at $5 \%$ in order to ensure a diverse population. Random pairs of chromosomes are selected for the recombination and mutation steps until the size of the next-generation population equals that of the initial population. My code written in Common Lisp for the EV selection strategy can be found in Appendix 1. 


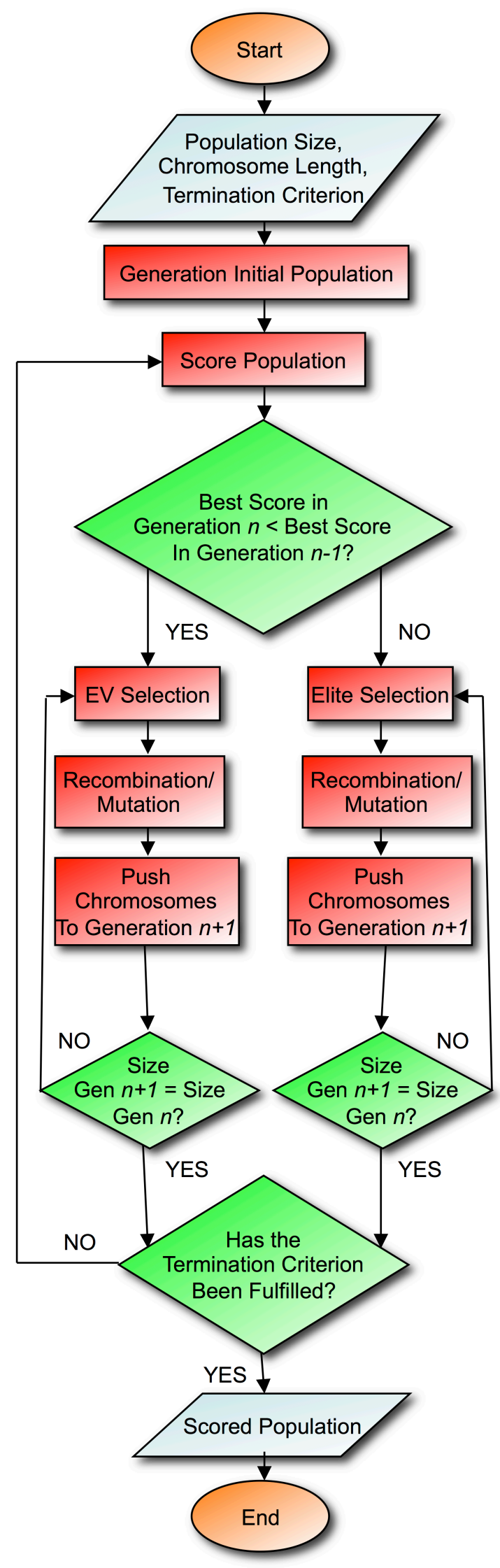

Figure 3.1. Genetic Algorithm Using Elite Variant Selection (White and Srivastava 2011).

A genetic algorithm using Elite Variant selection will require input as to the desired population size, chromosome length, and termination criterion. This diagram assumes that the objective function is to minimize the chromosome score. The first generation of the EV selection algorithm will always use Elite selection. Starting with the second generation, the algorithm will check every generation to see if Elite Variant or Elite selection should be used. The output of the algorithm will be a scored population of chromosomes, where the best scoring chromosome can be considered the optimized solution given the scoring metric and termination criterion. The use of Elite Variant selection will facilitate "leaps" in the evolution of the population of chromosomes while the use of Elite selection will give the population a means of escaping a local optimum. 
The algorithm then iterates and returns to the scoring step. The next-generation population becomes the current working population and is scored and sorted. At this point, EV selection is called. First, the score of the top-scoring chromosome in the current population is compared to the top-scoring chromosome of the previous generation. If the scores are the same, it is assumed that the top-scoring chromosome is currently at some local optimum. In this case, the algorithm continues to populate the next-generation population according to the Elite selection strategy, as in the first generation. If the top-scoring chromosome of the current generation is better (higher or lower depending on if the problem is a maximization or minimization problem), then the algorithm proceeds according to EV selection in an attempt to facilitate convergence along the current path. First, the top-scoring chromosome is copied to the next generation. In the case of an even population size, the first two top-scoring chromosomes are copied to the next generation. The rest of the next-generation population is made up of chromosome pairs containing one random chromosome and the best-scoring chromosome. These chromosome pairs are then recombined. Two random chromosome positions are selected at which genetic material is exchanged. Each part of the recombined chromosomes is also given a $5 \%$ chance to mutate.

The algorithm once again iterates to the next generation and continues to iterate until a specified amount of generations have been completed or a termination criterion has been reached. The critical decision point in each generation is whether or not the best-scoring chromosome in the working generation is better scoring than the bestscoring chromosome of the previous generation. If the scores are equal, then the nextgeneration population is determined according to the Elite selection strategy, while if the 
score has improved, the next-generation population is determined according to the EV selection strategy.

The purpose of the EV selection method is to facilitate "leaps" in the evolution of the population while at the same time maintaining its diversity and ability to search a broad range of the solution space as to not plateau at some local optimum. This is accomplished first by preserving genetic material that yields a good score in comparison to other genetic material and by also breeding that genetic material with other randomly selected chromosomes to possibly create even better chromosomes. Since the bestscoring chromosome is forced to breed with other randomly selected chromosomes in EV selection generations, the algorithm is pushed to continually improve at a rapid rate. At the same time, the algorithm can maintain its diversity by reverting back to the Elite selection strategy when an improvement is not discovered. The mutation rate is also set to $5 \%$, a high rate when compared to most genetic algorithm applications, in an effort to maintain adequate diversity.

\subsubsection{Application of Test Cases to Elite-Variant Selection Genetic Algorithm}

In an effort to compare Elite Variant selection to other commonly used selection methods, genetic algorithms using EV, Elite, Tournament, Proportional, and Linear Ranking selection were applied to multiple test problems taken from the literature.

Five test functions were used to evaluate the ability of genetic algorithms using each selection strategy to evolve to a global optimum (Oltean 2005). The functions used are listed in Table 3.1. Each algorithm in all five tests had a population size of 100 chromosomes and a chromosome length of 100 integers. The integers comprising the 
chromosomes were initialized randomly to values between -100 and 100 for Function Test 1, between -10 and 10 for Function Test 2, between -30 and 30 for Function Test 3, between -5 and 5 for Function Test 4, and between -500 and 500 for Function Test 5 . The optimal solution for all five test functions was a chromosome made up entirely of 0 's which would yield a score of 0 . The goal therefore was to minimize the chromosome score. Each algorithm was programmed to terminate after 25,000 generations. For each function test, 50 trials were conducted per selection strategy.

\begin{tabular}{|c|c|}
\hline \multicolumn{2}{|c|}{ Table 3.1: Genetic Algorithm Function Tests (Oltean 2005) } \\
\hline Function Test 1 & $F_{1}=\sum_{i=1}^{n}\left(x_{i}^{2}\right)$ \\
\hline Function Test 2 & $F_{2}=\sum_{i=1}^{n}\left|x_{i}\right|+\prod_{i=1}^{n}\left|x_{i}\right|$ \\
\hline Function Test 3 & $F_{3}=\sum_{i=1}^{n-1}\left(100 *\left(x_{i+1}-x_{i}^{2}\right)^{2}+\left(1-x_{i}\right)^{2}\right)$ \\
\hline Function Test 4 & $F_{4}=10 * n+\sum_{i=1}^{n}\left(x_{i}^{2}-10 * \cos \left(2 * \pi * x_{i}\right)\right)$ \\
\hline Function Test 5 & $F_{5}=\frac{1}{4000} \sum_{i=1}^{n}\left(x_{i}^{2}\right)-\prod_{i=1}^{n}\left(\cos \left(\frac{x_{i}}{\sqrt{i}}\right)\right)+1$ \\
\hline
\end{tabular}

Next, a satisfiability problem (De Jong and William 1991, Lardeux et al. 2006) was tested on each selection strategy. In this case, the desired solution was a binary string of 0 's and 1's specified by the user. One point was added to the score of a chromosome for each position that did not match the desired solution. For example, if position 29 in chromosome 39 contained a " 1 ", but position 29 of the solution contained a "0", one 
point was added to the score of chromosome 39. The goal was to minimize the chromosome score; a score of zero would signal that the solution had been found. In this study, the solution was randomly generated prior to every trial and each position in the initial population of chromosomes was initialized between 5 and 10 so that each trial was initialized at a sufficiently unsatisfactory solution. The population was again set to 100 chromosomes and the chromosome size was also set to 100 positions or alleles. It was again observed how quickly and how close a genetic algorithm with a specified selection strategy could evolve towards the optimal solution. The termination criterion was set to 25,000 generations. For each selection strategy, 50 trials were again carried out.

Finally, a complex problem involving the use of genetic algorithms to evolve Highly Active Antiretroviral Therapy (HAART) regimens for Human Immunodeficiency Virus (HIV) subjects was explored using EV as well as the other selection strategies under investigation. The problem constituted determining an optimum 200-day HAART dosage schedule for a theoretical subject described by a mathematical model. The model is given by Equations 3.1-3.5 and is schematically depicted in Figure 3.2 as a biomolecular reaction kinetic network (Wu and Zhang 2010).

$$
\begin{gathered}
\frac{d\left[T_{c}\right]}{d t}=k_{1}-k_{2}\left[T_{c}\right]-k_{9}(1-R T)\left[V_{s}\right]\left[T_{c}\right]-k_{10}\left[V_{r}\right]\left[T_{c}\right] \\
\frac{d\left[T_{s}\right]}{d t}=k_{9}(1-R T)\left[V_{s}\right]\left[T_{c}\right]-k_{3}\left[T_{s}\right] \\
\frac{d\left[T_{r}\right]}{d t}=k_{10}\left[V_{r}\right]\left[T_{c}\right]-k_{4}\left[T_{r}\right] \\
\frac{d\left[V_{s}\right]}{d t}=k_{5}\left(1-k_{11}\right)(1-P I)\left[T_{s}\right]-k_{7}\left[V_{s}\right]
\end{gathered}
$$




$$
\frac{d\left[V_{r}\right]}{d t}=k_{6}\left[T_{r}\right]+k_{5} k_{11}\left[T_{s}\right]-k_{8}\left[V_{r}\right]
$$

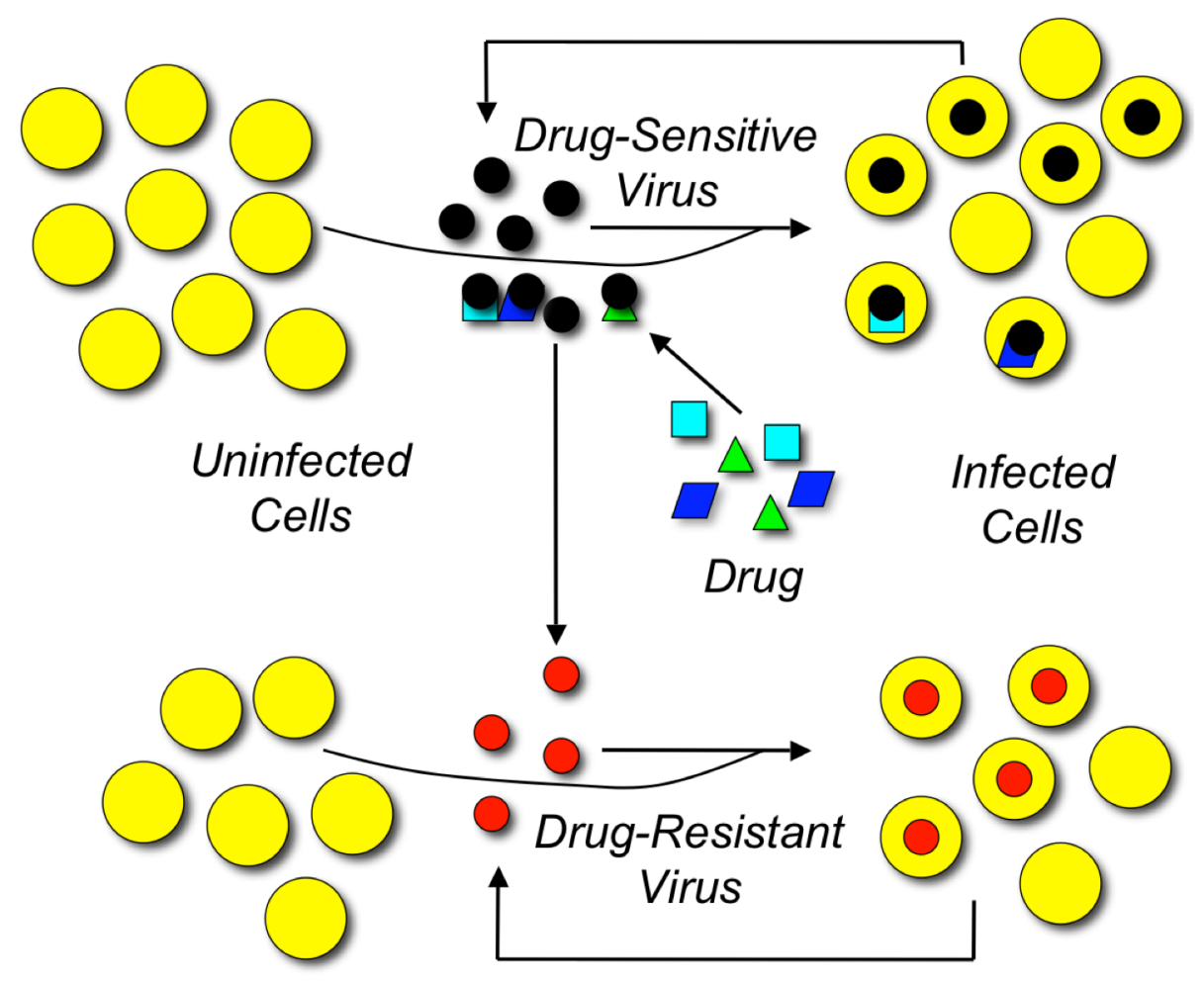

Figure 3.2. HIV Dynamic Model (based on Wu and Zhang 2010). In the presence of HIV, uninfected CD4+ T-cells can be infected by drug-sensitive (black dots) or drug-resistant (red dots) virus resulting in infected CD4+ T-cells. These infected T-cells then proceed to produce and release additional virus. Mutation in the drug-sensitive HIV can also result in new drug-resistant HIV. Highly Active Antiretroviral Therapy (HAART), which consists of two types of reverse transcriptase inhibitors (teal boxes and blue parallelograms) and a protease inhibitor (blue triangles), inhibits the ability of HIV to reproduce in infected cells rendering the virus inactive. All model components are subject to degradation pathways. 
In this model, [Tc] represents the concentration of uninfected CD4+ T-cells, [Ts] represents the concentration of CD4+ T-cells infected by drug-sensitive HIV, $[\mathrm{Tr}]$ represents the concentration of CD4+ T-cells infected by drug-resistant HIV, [Vs] represents the drug-sensitive HIV, and [Vr] represents the drug-resistant HIV. Uninfected $\mathrm{CD} 4+\mathrm{T}$-cells are constitutively produced (described by zero-order reaction parameter $\mathrm{k} 1$ ) and are subject to degradation (k2). They can also be lost due to infection by drugsensitive (k9) and drug-resistant (k10) HIV. Infection by drug-sensitive HIV is modulated by the presence of reverse transcriptase inhibitors (RT). CD4+ T-cells infected by drugsensitive HIV are the result of the infection of uninfected cells with drug-sensitive HIV and can be lost to degradation (k3). CD4+ T-cells infected by drug-resistant HIV are the result of the infection of uninfected cells with drug-resistant HIV and can also be lost to degradation (k4). Drug-sensitive HIV is produced by drug-sensitive HIV infected T-cells (k5) and is subject to degradation (k7). The production of new drug-sensitive HIV can be impeded by protease inhibitors (PI) and mutation to a drug-resistant strain (k11). Finally, drug-resistant HIV is produced by drug-resistant HIV infected T-cells (k6) and is also subject to degradation (k8). Drug-resistant HIV levels also increase when drug-sensitive HIV mutates to a drug-resistant strain. Both reverse transcriptase inhibitors and protease inhibitors are present in HAART. The model parameters are listed in Table 3.2. 


\begin{tabular}{|c|c|c|}
\hline \multicolumn{4}{|c|}{ Table 3.2. HIV Dynamic Model Parameters (Wu and Zhang 2010) } \\
\hline Parameter & Description & Base Value \\
\hline $\mathrm{k}_{1}$ & Growth rate of T cells & $10 \mathrm{cells} /\left(\mathrm{mm}^{3 *}\right.$ day) \\
\hline $\mathrm{k}_{2}$ & Death rate of T cells & $0.01 /$ day \\
\hline $\mathrm{k}_{3}$ & Death rate of T cells infected by sensitive virus & $0.02 /$ day \\
\hline $\mathrm{k}_{4}$ & Death rate of T cells infected by resistant virus & $0.01 /$ day \\
\hline $\mathrm{k}_{5}$ & Growth rate of sensitive virus & $8 /$ day \\
\hline $\mathrm{k}_{6}$ & Growth rate of resistant virus & $8 /$ day \\
\hline $\mathrm{k}_{7}$ & Natural death rate of sensitive virus & $30 /$ day \\
\hline $\mathrm{k}_{8}$ & Natural death rate of resistant virus & $30 /$ day \\
\hline $\mathrm{k}_{9}$ & Natural death rate of T cell infection by sensitive virus & $3^{*} 10^{-4} \mathrm{~mm}^{3} /($ virons day) \\
\hline $\mathrm{k}_{10}$ & Natural death rate of T cell infection by resistant virus & $5^{\star} 10^{-5} \mathrm{~mm}^{3} /($ virons \\
\hline $\mathrm{k}_{11}$ day $)$ \\
\hline
\end{tabular}

An optimal treatment will maintain the uninfected T-cell levels above 500 cells $/ \mathrm{mm}^{3}$ and the combined HIV titer below 50 virons $/ \mathrm{mm}^{3}$ while minimizing the amount of treatment administered. The bounds on the Tc and HIV titers are taken from Office of AIDS Research Advisory Council's definition effective HIV treatment (Panel 2009). The purpose of minimizing the total treatment dosage is to reduce the subject's risk of suffering dose-associated toxicities. Genetic algorithms have been used as a tool to optimize HAART regimens for theoretical subject (Castiglione et al. 2007). Genetic algorithms were therefore programmed using each selection method under investigation to optimize HAART treatment for HIV in the theoretical subject. The fitness of each chromosome was calculated by summing the penalties incurred by both failing to keep the Tc levels and HIV titers outside the respective bounds and by treatment dosage. Each chromosome consisted of 200 daily dosages of HAART ranging between $0 \%$ and $100 \%$ of full HAART dosage. The objective of each algorithm was to minimize the chromosome score. For each selection strategy, ten trials were conducted. The algorithms were carried out over 2,000 generations and the treatment score results as well as the effect of each treatment on the HIV model variables were compared to determine which 
algorithm provided the best treatment strategy.

\subsection{Results}

\subsubsection{Function Tests}

Utilization of the Elite Variant (EV) selection method resulted in significantly faster convergence to the global optima of Function Tests 1-4 as shown in Figures 3.3a-d. Although convergence to the global optimum never occurred for Function Test 5 after 25,000 generations, the use of Elite Variant selection yielded a significantly better scoring result, shown in Figure 3.3e.

Function Test 1 (see Figure 3.3a) displayed convergence of the genetic algorithms using EV and Elite selection to the global optimum, which is a string of 0's with a chromosome score of 0 . Use of EV selection resulted in convergence to the global optimum in all $50 \mathrm{EV}$ trials after 21,000 generations, with $98 \%$ of trials converging by generation 14,500. Using Elite selection, 94\% of trials reached the global optimum after 25,000 generations. In comparison to the EV method, $0 \%$ of Elite trials reached the global optimum after 14,500 generations. The Tournament, Proportional, and Linear Ranking selection algorithms all quickly reached local optima at chromosome scores of approximately 600, 1,800, and 40,000 respectively (Linear Ranking data not shown) and were not able to escape the local optima by generation 25,000 . None of the trials using these selection methods resulted in the optimal solution. 
A

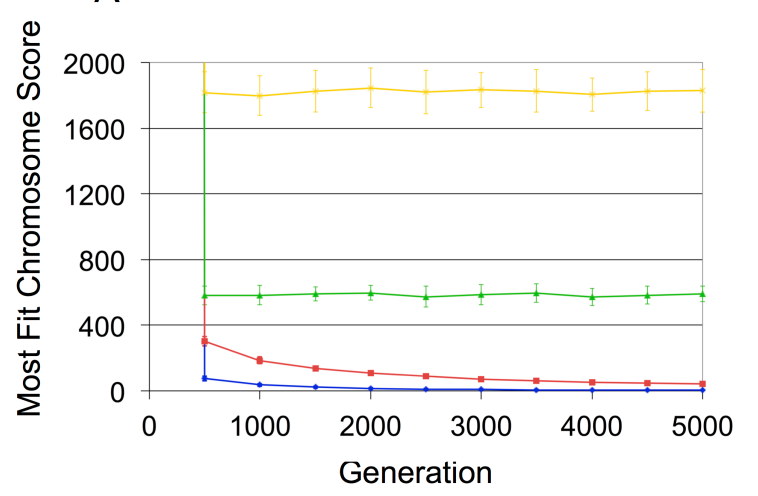

C

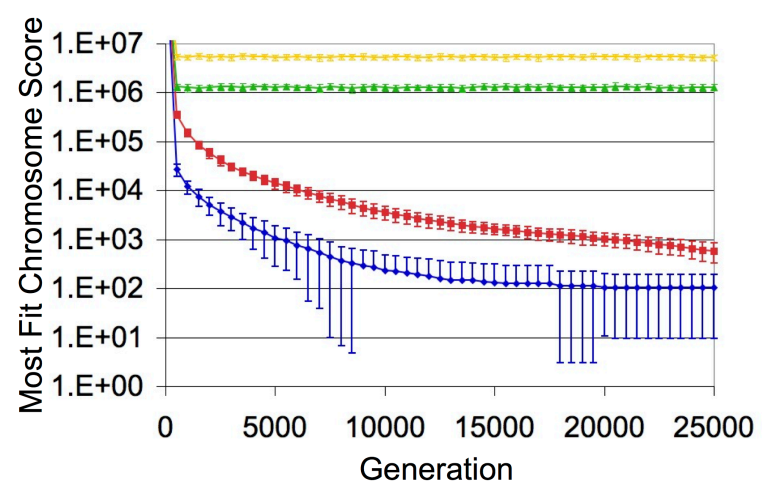

$E$
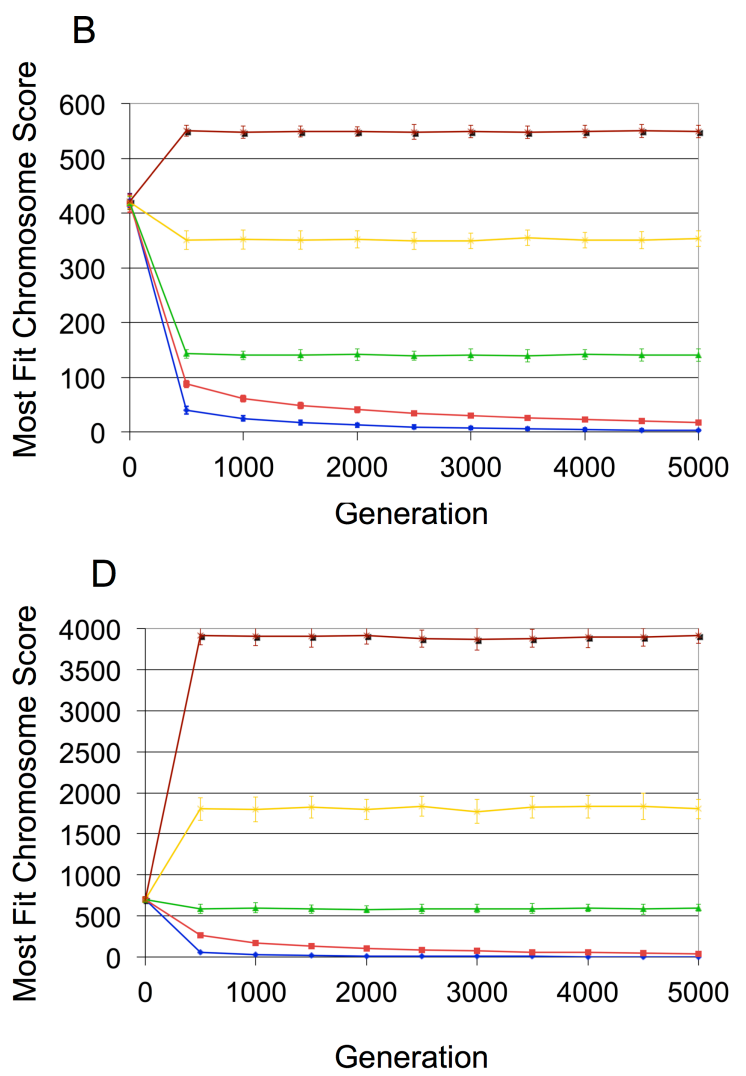

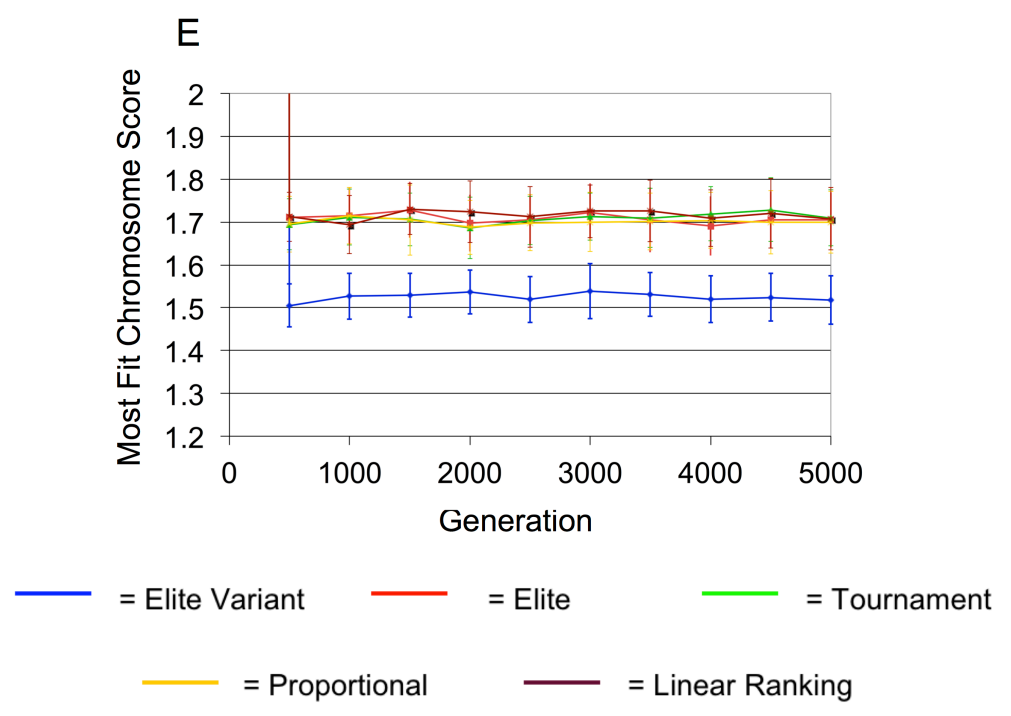

Figure 3.3. Evolution of Solutions in Function Tests. Genetic algorithms programmed to solve Function Tests 1 (A), 2 (B), 3 (C), 4 (D), and 5 (E) were carried out over 25,000 generations. The best scoring chromosome was plotted every 50 generations for genetic algorithms using EliteVariant (blue), Elite (red), Tournament (green), Proportional (gold), and Linear Ranking (brown) selection methods. For each test, 50 trials were carried out per algorithm. It was observed that the 
genetic algorithms using Elite Variant (EV) selection converged to better scoring solutions in less time than genetic algorithms using other selection methods. It was also observed that genetic algorithms using Tournament, Proportional, or Linear Ranking selection were not able to escape local optima by generation 25,000 for all Function Tests. Note: The error bars attached to the EV selection plot in Figure 3.3c extend beyond the graphical space and are therefore left unbounded. The error incurred in these trials reached values below zero and cannot be displayed on a logarithmic scale.

Function Test 2 (see Figure 3.3b) displayed a similar result to that of Function Test 1 as the genetic algorithms utilizing EV and Elite selection converged to the global optimum, while the genetic algorithms utilizing Tournament, Proportional, and Linear Ranking selection quickly reached local optima at approximate chromosome scores of 150,350 , and 550 respectively from which they were not able to escape by generation 25,000. The Linear Ranking selection algorithm, because it placed more weight on chromosome rank rather than chromosome score, quickly lost the top scoring chromosomes from the early generations and reached an inferior local optimum as compared to its starting point. Genetic algorithms using EV selection reached the global optimum after 18,500 generations in all 50 trials. Similarly, genetic algorithms using Elite selection reached the global optimum after 21,500 generations in all 50 trials. A more telling comparison is that $90 \%$ of the EV algorithms reached the optimal solution after 13,000 generations whereas only $24 \%$ of the algorithms using Elite selection reached the global optimum at this time.

The scale of chromosome scores in Function Test 3, as can be seen in Figure 3.3c, provided a challenge to each selection method. $40 \%$ of the genetic algorithm trials using 
EV selection were able to find the global optimum after 25,000 generations. No other genetic algorithm trials using other selection methods were able to find the global optimum by generation 25,000. Likewise, EV algorithms on average yielded a significantly better result after 25,000 generations than did algorithms using the other selection methods. Recalling that lower scores are desirable in this case, the average results after 25,000 generations were $102.5 \pm 93.0$ using EV selection, $582.6 \pm 251.1$ using Elite selection, $1.3 * 106 \pm 1.9 * 105$ using Tournament selection, $5.2 * 106 \pm 7.0 * 105$ using Proportional selection, and $3.5^{*} 108 \pm 6.1 * 107$ using Linear Ranking selection.

Function Test 4 also yielded a result similar to that of Function Tests 1 and 2, as can be seen in Figure 3.3d. Genetic algorithms using Tournament, Proportional, and Linear Ranking selection again reached local optima at approximately 580, 1,800, and 3,900 respectively, from which they were not able to escape by generation 25,000. Again, the Linear Ranking selection algorithm as well as the Proportional selection algorithm by maintaining too broad a search of the solution space quickly lost the top scoring chromosomes of the early generations and reached local optimums at chromosomes scores greater than that of the best early generation chromosome scores. All algorithm trials using EV and Elite selection converged to the global optimum by generation 25,000 . In contrast, at generation $11,500,92 \%$ of genetic algorithm trials using EV selection had converged to the global optimum while no trials using Elite selection had converged. In fact, the average chromosome score of a population in an algorithm using Elite selection at generation 11,550 was $10.5 \pm 3.0$.

Function Test 5 displayed the most peculiar behavior of all of the Function Tests, as can be seen in Figure 3.3e. The results of this test showed that genetic algorithms using 
Elite, Tournament, Proportional, and Linear Ranking selection converged along a very similar path while genetic algorithms using EV selection converged quickly to a more optimal solution. The average best solutions after 25,000 generations for algorithms using each selection method were $1.53 \pm 0.055$ for EV selection, $1.71 \pm 0.067$ for Elite selection, $1.72 \pm 0.062$ for Tournament selection, $1.69 \pm 0.065$ for Proportional selection, and $1.70 \pm 0.061$ for Linear Ranking selection. No trials were able to reach the global optimum in this test.

\subsubsection{Satisfiability Test}

Implementation of a satisfiability test, shown in Figure 3.4, also demonstrated the ability of the EV selection method to facilitate the convergence of a genetic algorithm to an optimal solution. Genetic algorithms using Tournament, Proportional, and Linear Ranking selection yielded results at local optimum chromosome scores of approximately 450, 1,500, and 3,400 respectively. Again, algorithms using these selection methods were not able to escape these local optima by generation 25,000. Conversely, algorithms using EV selection converged to the global optimum by generation 16,000 in all cases with $90 \%$ reaching this optimum by generation 12,500 . 

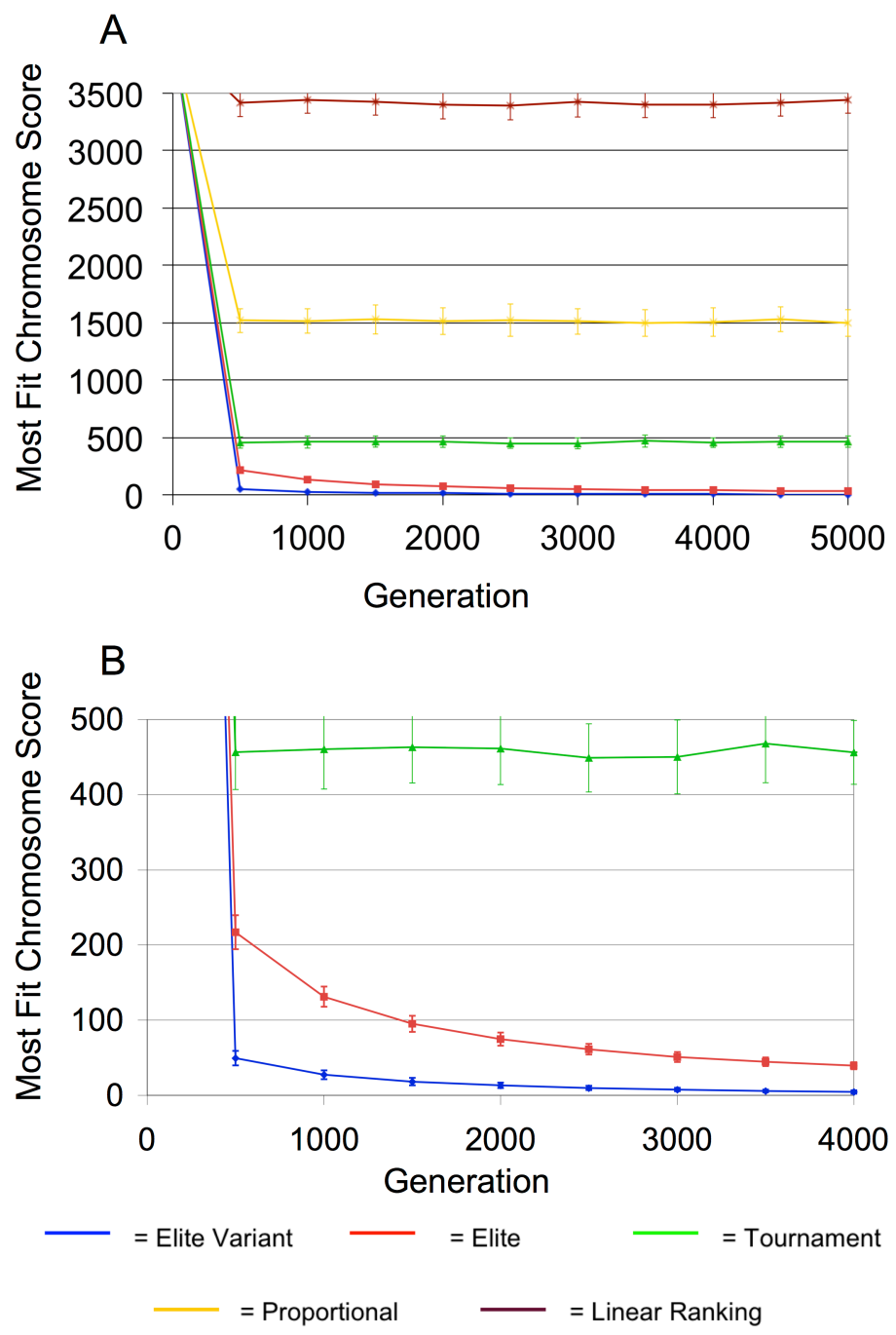

Figure 3.4. Evolution of Satisfiability Test Solutions. Scores of the most fit chromosomes were plotted every 50 generations for genetic algorithms using Elite Variant (EV) selection (blue), Elite selection (red), Tournament selection (green), Proportional selection (gold), and Linear Ranking selection (brown) to solve a satisfiability problem. The algorithm was carried out for 25,000 generations (A), with the fastest convergence occurring in the first 4,000 generations (B). Each algorithm was carried out 50 times. The genetic algorithm using EV selection was able to solve the satisfiability problem in fewer generations than any genetic algorithm using another selection method. Genetic algorithms using Tournament, Proportional, or Linear Ranking selection were not able to solve this satisfiability problem in 25,000 generations. 
Genetic algorithms using Elite selection also exhibited desirable convergence, with $96 \%$ of the trials reaching the global optimum by generation 25,000 . Elite selection trials again did not converge as quickly as EV selection trials. In comparison, at generation 16,000 when all EV selection trials had converged, only $40 \%$ of the Elite selection trials had converged to the global optimum.

\subsubsection{HAART Optimization}

Genetic algorithms using the selection strategies under investigation were implemented to optimize Highly Active Antiretroviral Therapy (HAART) regimens for a theoretical Human Immunodeficiency Virus (HIV) subject. Figure 3.5 shows the fitness of the best scoring chromosomes plotted over 2,000 generations for each case. The objective once again was to minimize the chromosome score. Genetic algorithms using $\mathrm{EV}$ and Elite selection produced better scoring chromosomes in each generation as compared to genetic algorithms using Tournament, Proportional, and Linear Ranking selection. In fact, the Proportional selection and the Linear Ranking selection algorithms once again reached local optimums at scores above that of the best scoring early generation chromosomes due to too broad a search of the solution space. After 2,000 generations, the genetic algorithm using EV selection produced an average top scoring chromosome score of $2,475.23 \pm 18.42$ while the genetic algorithm using Elite selection produced an average top scoring chromosome score of 2,523.34 \pm 22.77 . In comparison, the average top scoring chromosome score using EV selection was, within error, the same as the top scoring chromosome using Elite selection between generations 0 and 1,700. Even so, the EV selection algorithm maintains a clear advantage over the Elite selection 
algorithm in regards to convergence time as the average result of the Elite selection algorithm after 2,000 generations was obtained by the EV selection algorithm after only 150 generations.

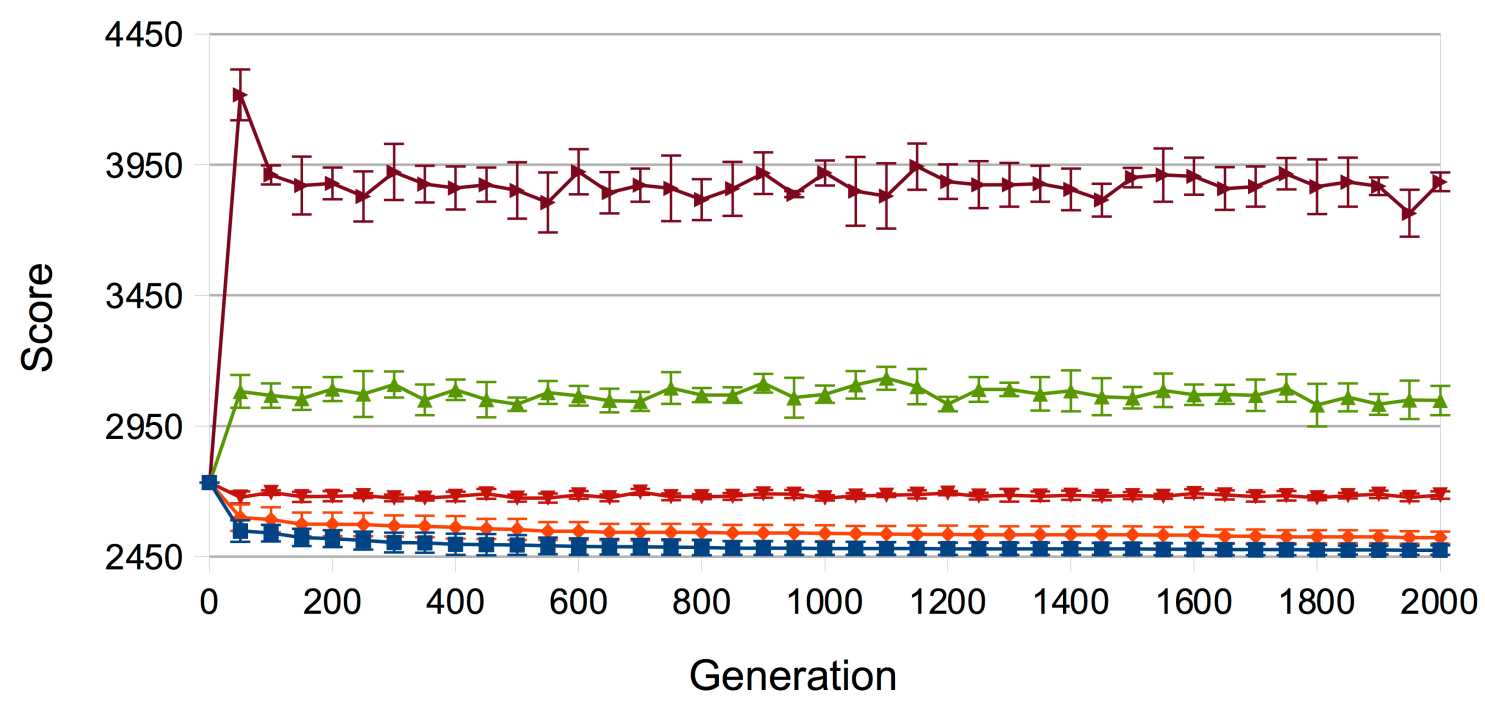

$\rightarrow$ Elite Variant $\rightarrow$ Elite $\rightarrow$ Tournament $₫$ Proportional $\rightarrow$ Linear Ranking

Figure 3.5. Evolution of Highly Active Antiretroviral Therapy (HAART) for Treatment of Human Immunodeficiency Virus (White and Srivastava 2011). Genetic algorithms using EV (blue), Elite (orange), Tournament (red), Proportional (green), and Linear Ranking (brown) selection were programmed to optimize HAART over 2,000 generations for treatment of a theoretical case of HIV. The score of the best scoring treatment was plotted every 50 generations. Each algorithm was carried out 10 times. The genetic algorithm using EV selection was able to evolve a more optimal HAART regimen after 2,000 generations than the genetic algorithms using the other selection methods. In fact, the average score of the top scoring treatment as determined using Elite selection after 2,000 generation was equal to the average score of the top scoring treatment as determined using EV selection after only 150 generations. 
A

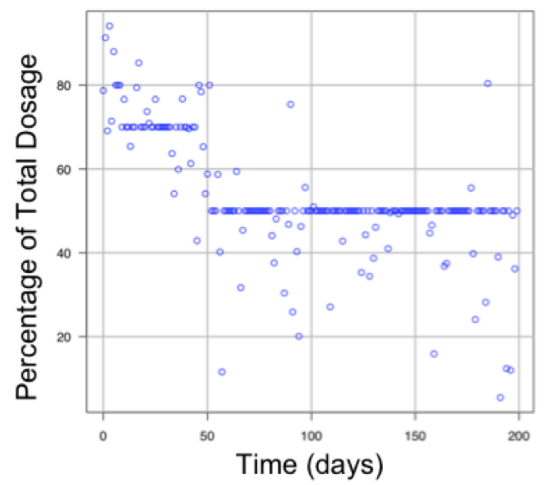

C

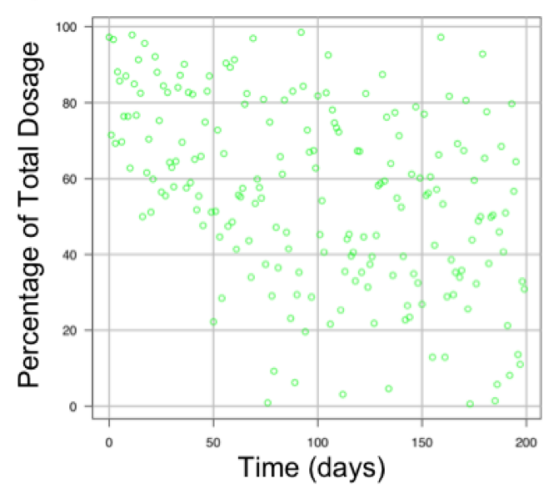

B

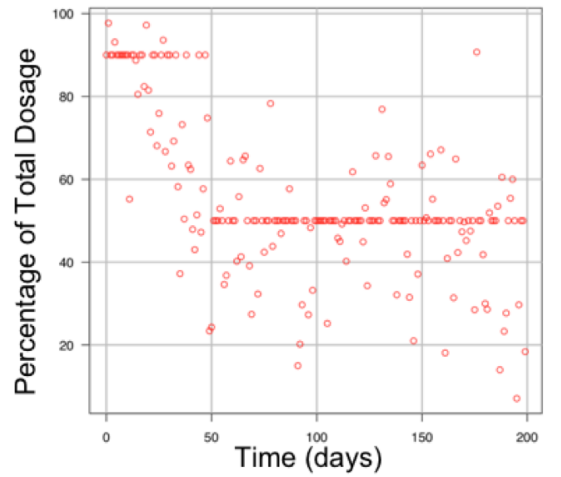

D

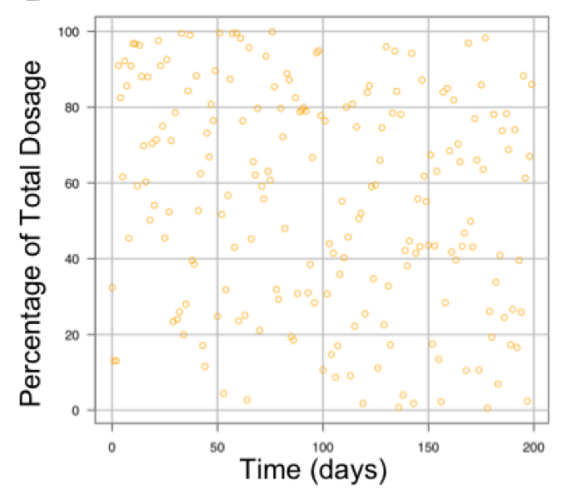

$\mathrm{E}$

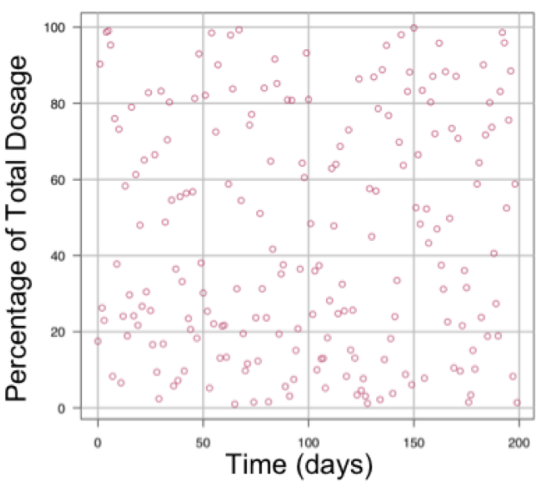

$\longrightarrow=$ Elite Variant $\quad-$ Elite $\quad-$ Tournament

$=$ Proportional $\quad-\quad=$ Linear Ranking

Figure 3.6. Optimized Highly Active Antiretroviral Therapy (HAART) Regimens (White and Srivastava 2011). After 2,000 generations, the top scoring treatment was taken from each genetic algorithm and used to treat a theoretical case of HIV. The HAART regimen as determined by the genetic algorithm using EV selection (A, blue) suggested on average a daily dose of $70 \%$ from day 0 to day 50 followed by a decrease to a daily dose of $50 \%$ from day 50 to day 200 with 
some fluctuation in dose. Similarly, the HAART regimen as determined by the genetic algorithm using Elite selection (B, red) suggested on average a daily dose of $90 \%$ from day 0 to day 50 followed by a decrease to a daily dose of $50 \%$ from day 50 to day 200 with some fluctuation in dose. HAART regimens as determined by genetic algorithms using Tournament (C, green), Proportional (D, gold), and Linear Ranking (E, brown) selection suggest a highly variable dosage from day to day over the course of the 200-day treatment period.

The HAART regimens evolved using genetic algorithms with different selection strategies are shown in Figures 3.6a-e. It was observed that HAART dosages optimized using Tournament, Proportional, and Linear Ranking selection fluctuated drastically each day. This result demonstrated the inability of the genetic algorithms using these selection methods to home in on an optimum HAART regimen. Conversely, HAART dosages optimized using EV and Elite selection were more consistent. In general, the HAART regimen optimized using EV selection resulted in daily dosages of $70 \%$ from day 0 to day 50 and daily dosages of $50 \%$ from day 50 to day 200 with some variation. The HAART regimen optimized using Elite selection resulted in the same pattern with the exception of daily dosages of $90 \%$ from day 0 to day 50 . The percentage of the total possible HAART dosage administered over 200 days was determined to be $53.28 \%$ using EV selection, $54.70 \%$ using Elite selection, 56.08\% using Tournament selection, 55.47\% using Proportional selection, and 44.85\% using Linear Ranking selection. 

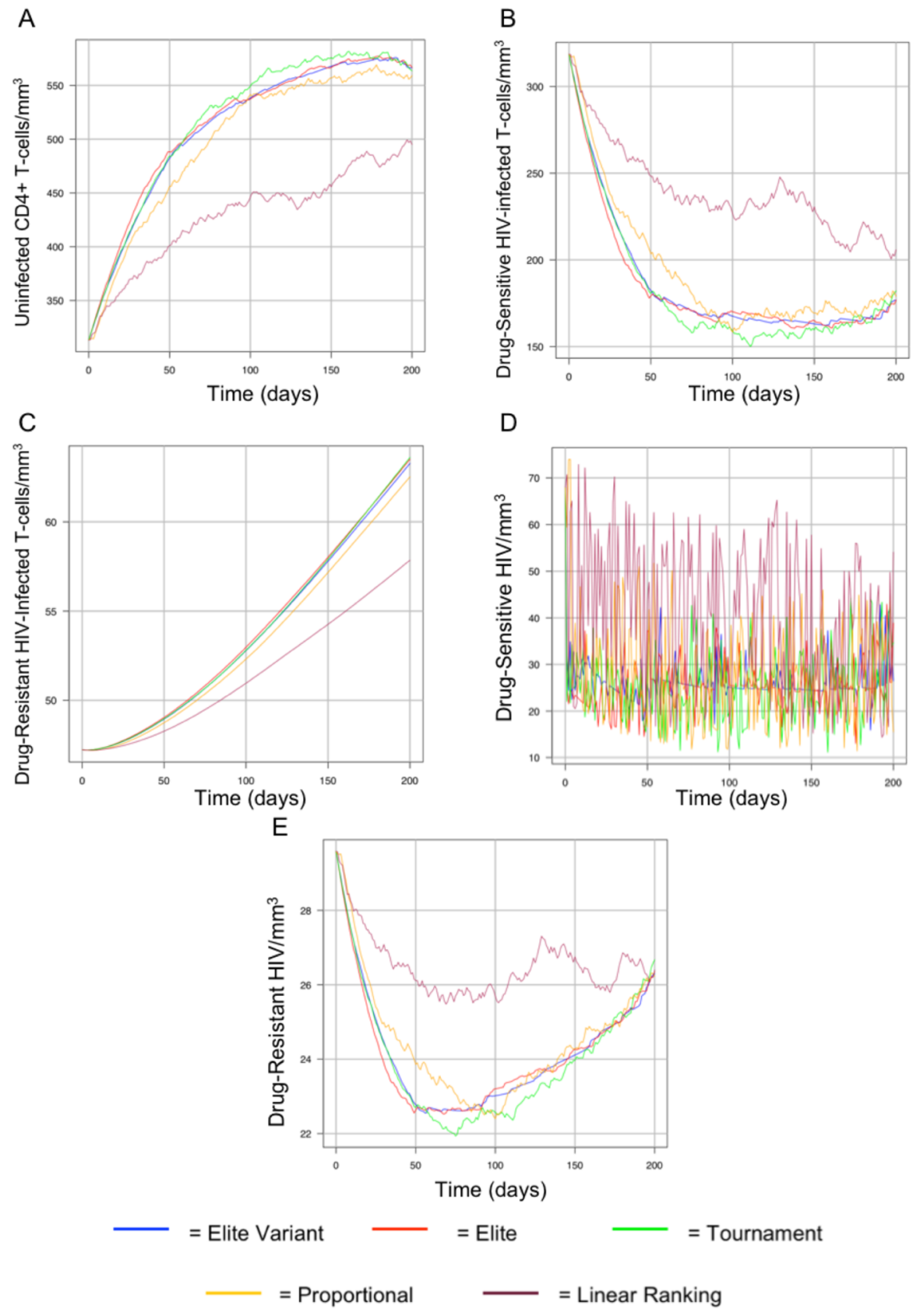

Figure 3.7. Effect of Optimized HAART on HIV Model Variables (White and Srivastava 2011). HAART as determined by genetic algorithms using EV (blue), Elite (red), Tournament (green), Proportional (gold), and Linear Ranking (brown) selection was applied to the HIV model over a 200 days period. The effects of HAART on uninfected T-cell (A), drug-sensitive HIV infected T-cell (B), drug-resistant HIV infected T-cell (C), drug-sensitive HIV (D), and drugresistant HIV (E) levels are shown. HAART as determined using EV, Elite, and Tournament 
selection resulted in a similar effect on all model variables with the exception of drug-sensitive HIV being less controlled using HAART determined using Tournament selection. HAART determined using Linear Ranking selection resulted in less control of the infected and a slower rebound of the uninfected T-cell levels. Conversely, less drug-resistant HIV and drug-resistant HIV infected T-cells accumulated in this case.

Figures 3.7a-e show the results of each HAART regimen on the model variables. In Figure 3.7a, it was observed that HAART regimens produced by genetic algorithms using EV, Elite, and Tournament selection strategies resulted in an increase in uninfected T-cell (Tc) levels to the goal of 500 cells $/ \mathrm{mm}^{3}$ by day 60 . The HAART regimens produced by genetic algorithms using Proportional and Linear Ranking selection also met this goal, but at a later time. HAART determined by Proportional selection met the Tc goal around day 75 while HAART determined by Linear Ranking selection met the same goal around day 200. Figures 3.7b and 3.7c display a similar result for drug-sensitive (Ts) and drug-resistant (Tr) HIV infected T-cells for cases when HAART was determined by genetic algorithms using EV, Elite, Tournament, and Proportional selection. HAART determined by a genetic algorithm using Linear Ranking selection resulted in a higher concentration of drug-sensitive and a lower concentration of drug-resistant cells. Following these results, HIV infection was less controlled by the HAART regimen determined using Linear Ranking selection, as illustrated in Figures 3.7d and 3.7e. Indeed, Figure 3.7d showed that all HAART regimens resulted in fluctuation of drugsensitive virus as a result of fluctuation in treatment dosages. Because treatment dosage was more variable in Linear Ranking, Proportional, and Tournament selection generated HAART, Vs titers fluctuate to a greater extent and less control is maintained over levels 
of drug-sensitive virus.

\subsection{Discussion}

The results of the Function Tests, Satisfiability Test, and HIV/HAART Test clearly demonstrate the advantages of using Elite Variant selection when using genetic algorithms to solve these problems. In all cases, algorithms using EV selection were able to arrive at better scoring solutions in a smaller amount of time, making it an attractive option when trying to quickly determine or estimate the solution of a complex problem.

EV selection demonstrates through these tests that it is not only able to converge quickly to an optimum, but it is also able to completely bypass local optima that other selection strategies spend significant time attempting to escape. Both Elite and EV selection balance the broad probing of the solution space exhibited by Tournament, Proportional, and Linear Ranking selection with a mechanism for keeping wellperforming genetic material while effectively pursuing the objective function. EV selection takes this methodology one step further by forcing the population quickly to a local optimum while also giving the algorithm a way to escape the local optimum. The algorithm reverts back to the Elite selection strategy while at a local optimum and then switches back to EV selection upon escape facilitating "leaps" in the evolution of the population.

In the examples of the Function Tests and the satisfiability test, the capabilities of EV selection are demonstrated. Following these results, the HIV/HAART test demonstrated the potential of EV selection in genetic algorithms to arrive at better solutions in a shorter period of time with the result being a more optimal treatment for a 
real disease. If a particular HIV subject could be modeled by a series of differential equations describing the key variables at play, a genetic algorithm using EV selection would be able to offer a HAART regimen that would more effectively treat that subject according to the constraints on uninfected T-cell levels, combined HIV titers, and HAART dosage. In the theoretical case presented in this paper, the HAART regimen produced by the genetic algorithm using EV selection was able to elevate the concentration of uninfected T-cells as quickly as the Elite, Tournament, and Proportional HAART regimens. It was also able to control the combined HIV titers as well as these other HAART regimens. What sets the EV HAART regimen apart from these other treatments is that it was able to achieve these results while administering a smaller dosage of HAART reducing the subject's exposure to the drug and chance of suffering doseassociated toxicities. Conversely, the Linear Ranking HAART regimen administered a smaller amount of HAART than did the EV HAART regimen. In doing so, the Linear Ranking HAART regimen was not able to bring the uninfected T-cell levels back up to 500 cells $/ \mathrm{mm}^{3}$ nearly as fast as the EV HAART regimen was able to. It also maintained far less control over the level of HIV virus. Because of these behaviors, the EV HAART regimen was the best scoring of all the HAART regimens.

As a result, Elite Variant selection, when used as the selection method in genetic algorithms, can result in better, faster solutions. It is advised, as any problem contains its own subtleties, that all selection methods should be tested when using a genetic algorithm to evolve a solution to a problem in case that particular problem is well suited to one of the other commonly used selection methods. A benefit of this algorithm, besides providing more accurate solutions to complex problems, is that the process of carrying 
out genetic algorithms will be less computationally intensive thereby expanding a researcher's capability to evolve solutions to more complex problems as well as carry out a greater amount of trials in a smaller amount of time. Applications of this algorithm will be beneficial in multiple domains in which genetic algorithms are useful, ranging from traditional optimization to classification technology in fields encompassing medicine, logistics, finance, etc. 


\section{CHAPTER 4}

OPTIMIZING HIV-1 TREATMENT VIA A SYSTEMS BIOLOGY APPROACH 


\subsection{Introduction}

A major goal of developing mathematical models of diseases is to understand the underlying pathology. One of the benefits arising from such a quantitative understanding is the potential to develop and optimize treatment strategies that maximize health benefits while minimizing secondary toxicity effects.

It is possible to capitalize upon recent advances in systems biology to implement this strategy. By systematically perturbing the biological network underlying the disease, it is possible to monitor the gene, protein, and informational pathway responses; integrate these data; and ultimately, formulate mathematical models describing the structure of the system (Ideker et al. 2001, van der Greef et al. 2004). Systems biology provides an impactful foundation for analysis of the comprehensive datasets that are needed in order to develop robust kinetic models. The technology also permits verification against experimental results. Ideally, the resulting model may be used to predict progression of the disease (Bogojeska et al. 2010, Burg et al. 2009, Castiglione and Paci 2010, Degon et al. 2008, de Sousa et al. 2010, Guedj et al. 2010, Hoffmann et al. 2002, Itakura 2010, Perelson et al. 1996, Smith and Ribeiro 2010, Srivastava et al. 2002, von Kleist et al. 2010, Wendelsdorf et al. 2010, Yang et al. 2007). It is also possible to identify, develop, and optimize treatment strategies in a mathematically rigorous fashion.

HIV-1 is a key disease that has received significant interest from the modeling community (Aviran et al. 2010, Bonhoeffer et al. 1997(b), Nowak and May 2000, Perelson et al. 1996, Perelson 2002). In recent years, HIV-1 treatments have significantly reduced AIDS-related mortalities and have enhanced long-term disease control (Dybul et al. 2001, Fagard et al. 2003, Powderly 2002, Ruiz et al. 2001). These benefits have been 
realized in large part via use of highly active antiretroviral therapy (HAART) in clinical practice. The HAART strategy consists of treating the disease with a combination of antiretroviral drugs. Despite advances in efficacy over prior generation treatments, HAART is associated with risk of serious side effects that include cardiovascular disease (Domingos et al. 2009), nephrotoxic effects (Izzedine et al. 2009), and oral toxicities (Nittayananta et al. 2010). Up to $16 \%$ of patients require modification or discontinuation of their HAART regimen due to drug-related toxicities (Mocroft et al. 2005).

In an attempt to reduce incidence and severity of these side effects, clinical trials have explored various methods of reducing drug exposure by structured treatment interruption (STI). In STI, HAART is discontinued and resumed at pre-defined time intervals in an effort to control the viremia while concurrently administering the lowest possible dose of HAART in order to reduce toxicity (Castiglione et al. 2007, Dybul et al. 2001, El-Sadr et al. 2006, Fagard et al. 2003, Garcia et al. 2001, Kilby et al. 2000, Ortiz et al. 2001, Ruiz et al. 2001). Published STI regimens that were analyzed include 8 weeks on -2 weeks off $(8 \mathrm{w} / 2 \mathrm{w})$ STI (Fagard et al. 2003) and 1 week on -1 week off (1w/1w) STI (Castiglione et al. 2007, Dybul et al. 2001). These studies and their outcomes are listed in Table 4.1. Other STI regimens which have been studied include 1 month on -1 month $(1 \mathrm{~m} / 1 \mathrm{~m})$ off STI (Ortiz et al. 2001) and SMART STI (El-Sadr et al. 2006). Unlike standard STI that discontinues and resumes treatment at pre-defined timepoints, SMART STI prescribes the initiation of treatment when the $\mathrm{CD} 4^{+}$T-cell concentration falls below 250,000 cells $/ \mathrm{mL}$ and the discontinuation of treatment when the $\mathrm{CD}^{+} \mathrm{T}$-cell concentration recovers to 350,000 cells $/ \mathrm{mL}$. 
Despite their theoretical promise STI strategies have been largely unsuccessful. Thus there remains a need to define safe and effective "optimal" HAART strategies for large and diverse clinical cohorts. Treatment that is optimized to an individual may permit more effective control of that individual's HIV disease while concurrently reducing total HAART exposure and associated toxicities. To this end the goal of this study was to design an optimized dosing schema, based on an established mathematical model of HIV, for minimizing HAART exposure while maintaining durable virologic suppression. Results were compared to clinically reported results of standard HAART and STI dosing regimens to further validate the conceptual framework.

\begin{tabular}{|l|c|c|}
\hline \multicolumn{3}{|c|}{ Table 4.1. Total HAART Dosage Prescribed By Each Treatment Regimen } \\
\hline $\begin{array}{l}\text { Description of Treatment } \\
\text { Regimen }\end{array}$ & $\begin{array}{l}\text { HAART Administered } \\
\text { (\% of maximum } \\
\text { dosage) }\end{array}$ & $\begin{array}{l}\text { \% of Subjects Achieving Viral } \\
\text { Suppression After Treatment } \\
\text { Period }\end{array}$ \\
\hline Optimized HAART & $78.1 \%$ & N/A \\
\hline Standard HAART & $100.0 \%$ & $90 \%$ \\
\hline 1 week on - 1 week off STI & $50.0 \%$ & $80 \%$ \\
\hline 8 weeks on - 2 weeks off STI & $83.3 \%$ & $77.5 \%$ \\
\hline
\end{tabular}

\subsection{Methods}

\subsubsection{Model Selection}

There is a substantive literature pertaining to development of models describing HIV-1 (Aviran et al. 2010, Bonhoeffer and Nowak 1997, Bonhoeffer et al. 1997(b), Bonhoeffer et al. 2000, Burg et al. 2009, Herz et al. 1996, Liang et al. 2010, Nowak et al. 1997, Nowak and May 2000, Perelson et al. 1996, Perelson 2002, Prosperi et al. 2009, Ribeiro et al. 2010, von Kleist et al. 2010, Wu et al. 2010, Wu and Zhang 2010, Zeng and Yang 2010). The HIV-1 dynamic model of Perelson and Nelson (Perelson and Nelson 1999) shown in Figure 4.1 was selected as the basis for this work because it 


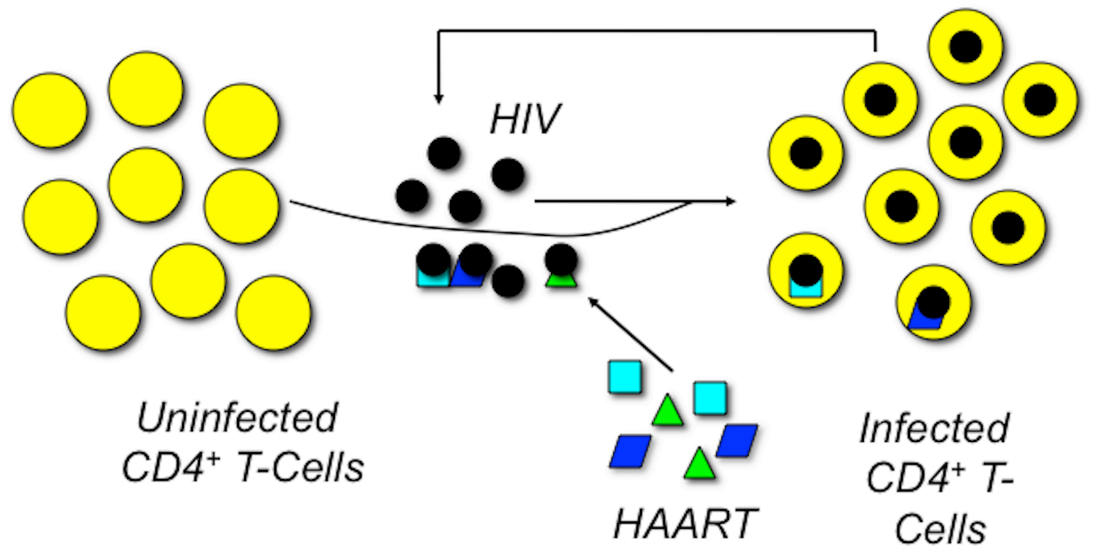

Figure 4.1. HIV Dynamic Model (based on Perelson and Nelson 1999). In the presence of HIV, uninfected CD4+ T-cells can be infected by HIV (black dots) resulting in infected CD4+ Tcells. The infected T-cells then proceed to produce and release additional virions. Highly Active Antiretroviral Therapy (HAART), which consists of nucleoside analog and non-nucleoside reverse transcriptase inhibitors (teal boxes and blue parallelograms) and a protease inhibitor (green triangles), inhibits the ability of HIV to reproduce in infected cells rendering the virus inactive. All model components are subject to degradation pathways.

included the action of reverse transcriptase inhibitors and protease inhibitors, both of which predominantly comprise HAART regimens. The model is quantitatively described via a set of ordinary differential equations presented in Equations 4.1-4.3.

$$
\begin{aligned}
& \frac{d[T]}{d t}=k_{1}-k_{2} * T-k_{3} *(1-R T) * V * T \\
& \frac{d\left[T_{I}\right]}{d t}=k_{3} *(1-R T) * V * T-k_{4} * T_{I} \\
& \frac{d[V]}{d t}=k_{4} * k_{5} *(1-P I) * T_{I}-k_{6} * V
\end{aligned}
$$


In this model, $\mathrm{T}$ represents the concentration of uninfected $\mathrm{CD} 4^{+} \mathrm{T}$-cells, $\mathrm{T}_{\mathrm{I}}$ represents the concentration of $\mathrm{CD}^{+}{ }^{+} \mathrm{T}$-cells infected by HIV, and V represents the HIV concentration. Uninfected $\mathrm{CD}^{+} \mathrm{T}$-cells are constitutively produced (described by zeroorder reaction parameter $\mathrm{k}_{1}$ ) and are subject to degradation $\left(\mathrm{k}_{2}\right)$. They can also be depleted due to infection by HIV $\left(\mathrm{k}_{3}\right)$. Infection by HIV is modulated by the presence of reverse transcriptase inhibitors (RT). $\mathrm{CD} 4{ }^{+}$T-cells infected by HIV are the result of the infection of uninfected cells with HIV $\left(\mathrm{k}_{3}\right)$ and can be lost to degradation $\left(\mathrm{k}_{4}\right)$. HIV is produced by HIV infected T-cells $\left(\mathrm{k}_{4}, \mathrm{k}_{5}\right)$ and is also subject to degradation $\left(\mathrm{k}_{6}\right)$. The production of new, drug-sensitive HIV can be impeded by protease inhibitors (PI). The average or baseline case parameters were derived from the literature (Perelson and Nelson 1999) and are listed in Table 4.2.

\begin{tabular}{|l|l|l|}
\hline \multicolumn{3}{|l|}{ Table 4.2. HIV Dynamic Model Parameters (Perelson and Nelson 1999) } \\
\hline Parameter & Description & Base Value \\
\hline $\mathrm{k}_{1}$ & Growth rate of T cells & 10,000 cells/(mL*day) \\
\hline $\mathrm{k}_{2}$ & Death rate of T cells & $0.01 /$ day \\
\hline $\mathrm{k}_{3}$ & Infection of T cells by HIV & $5^{\star} 10^{-1} \mathrm{~mL} /\left(\mathrm{copies}{ }^{*}\right.$ day) \\
\hline $\mathrm{k}_{4}$ & Death rate of T cells infected by virus & $0.5 /$ day \\
\hline $\mathrm{k}_{5}$ & Burst size/Virions released by lysed cell & 16.75 copies/cell \\
\hline $\mathrm{k}_{6}$ & Natural death rate of sensitive virus & $3.1 /$ day \\
\hline
\end{tabular}

The Perelson and Nelson model accounted for two types of CD4+ T-cells (uninfected, and infected) and a general strain of HIV. These sub-populations of CD4+ Tcells as of yet cannot be measured clinically and therefore the specific concentrations of these variables as reported by the model cannot be verified clinically. The predictions for each of these three variables made by the model were taken to be a reasonable 
approximation of HIV infection. We anticipate the advances in technology that will allow for the differentiation of these subpopulations of CD4+ T-cells in the clinical setting.

When applying HAART in this model, it was assumed that $50 \%$ of the dose contained a protease inhibitor and the other $50 \%$ of the dose contained a mixture of two reverse transcriptase inhibitors; this assumption is consistent with most HAART dosing recommendations (Panel 2009). In the model this was described by setting both RT and PI equal to 0.45 , thereby quantifying the inhibition of the pathways describing HIV infection and propagation associated with these drugs. In this study, HAART dosages were reported daily on a scale that ranged from $0 \%$ (null dose) to $100 \%$ (full dose). A full dose $(100 \%)$ in this case refers to the maximum dosage that can be administered. Outcomes evaluated for each dosing strategy was the range of successful treatment coverage (ROC), Equation 4.4, defined as the ability of the treatment to suppress the HIV titer below 50 copies $/ \mathrm{mL}$ at the 48 -week time point at the conclusion of the simulation. The 48-week time frame was chosen as it is used as a common benchmark for determining the efficacy of a HIV treatment regimen (Eron et al. 2006, Madruga et al. 2007, Sanne et al. 2003).

$$
R O C=\frac{\# \text { SubjectsForWhich }[\text { HIV }]_{t=48 w e e k s}<50 \text { copies } / m L}{\text { Total } \# \text { SubjectsInCohort }}
$$

\subsubsection{Optimization of Highly Active Antiretroviral Therapy (HAART)}

A 48-week (336-day) HAART regimen was optimized for the average subject based on Equations $4.1-4.3$ using the gPROMS software. gPROMS employs both single-shooting and multiple-shooting dynamic optimization methods to converge upon 
an optimal solution. The average subject's biochemical indicators, described by Equations 4.1-4.3, were allowed to equilibrate after being initiated from the initial conditions listed in Table 4.3. The equilibrium values for each of the 3 variables, listed in Table 4.4, were used as the initial conditions for HAART optimization. The optimized HAART regimen was described by a list of daily HAART dosages ranging from $0 \%$ to $100 \%$. The objective function, defined by Equation 4.5, was minimization of the total dosage of HAART. The constraint imposed was that the HIV titer remained below 50 copies $/ \mathrm{mL}$. The bound on the HIV titer was chosen based upon the detection limit and target for suppression of HIV RNA (Panel 2009).

$$
\begin{array}{r}
\min Z=\sum_{t=0}^{336} \frac{H A A R T(t)}{100 \%} \quad \begin{array}{r}
\text { s.t. }[V] \in[0,50) \\
0 \leq H A A R T \leq 100
\end{array}
\end{array}
$$

\begin{tabular}{|l|l|}
\hline Table 4.3. Average Subject's Initial Conditions, Pre-Treatment \\
\hline Variable & Initial Condition \\
\hline$T$ & $1,000,000$ cells $/ \mathrm{mL}$ \\
\hline$T_{I}$ & 0 cells $/ \mathrm{mL}$ \\
\hline$V$ & 100 copies $/ \mathrm{mL}$ \\
\hline
\end{tabular}

\begin{tabular}{|l|l|}
\hline \multicolumn{2}{|l|}{ Table 4.4. Initial Conditions at the Initiation of Treatment Optimization } \\
\hline Variable & Initial Condition \\
\hline$T$ & $462,838 \mathrm{cells} / \mathrm{mL}$ \\
\hline$T_{1}$ & $223,368 \mathrm{cell} / \mathrm{sL}$ \\
\hline$V$ & $557,195 \mathrm{copies} / \mathrm{mL}$ \\
\hline
\end{tabular}




\subsubsection{Comparison of Optimized HAART to Standard HIV-1 Treatments Administered to}

\section{Computer-Generated Cohorts}

Seven groups of 1,000 HIV positive subject computer-generated cohorts with different degrees of inter-patient variation were simulated. Each computer-generated cohort was comprised of 1,000 computer-generated (in silico) subjects. Specifically, parameter values in the first group were allowed to vary randomly by as much as $4 \%$ from the baseline. Parameter values in the remaining six groups were allowed to vary by as much $7 \%, 10 \%, 20 \%, 25 \%, 30 \%$, or $40 \%$. Smaller percentage parameter variation indicated a more homogeneous population, while higher percentage parameter variation indicated a cohort with a more diverse immune response. Each computer-generated cohort was treated with standard and STI HAART regimens, including consistent maximum or standard HAART dosage, an optimized HAART regimen, $8 \mathrm{w} / 2 \mathrm{w}$ STI (Fagard et al. 2003), and 1w/1w STI (Castiglione et al. 2007, Dybul et al. 2001). Ten in silico cohorts were evaluated at each measure of inter-patient variation for each of the treatment regimens. Simulated treatment was initiated when the uninfected $\mathrm{CD}^{+}{ }^{+} \mathrm{T}$-cell count fell below 500,000 cells $/ \mathrm{mL}$. Standard HAART and optimized HAART were simulated for 48 weeks after its initiation. In order to compare results in this in silico study directly to past clinical trials, $8 \mathrm{w} / 2 \mathrm{w}$ STI was simulated for 40 weeks (Fagard et al. 2003) and 1w/1w STI was simulated for 68 weeks (Dybul et al. 2001). Subjects enrolled in these clinical trials had undetectable $([\mathrm{HIV}]<50$ copies $/ \mathrm{mL}$ ) viral loads. To closely replicate these trials, in silico subjects were treated with full treatment until the

concentration of HIV fell below 50 copies $/ \mathrm{mL}$, after which the STI was initiated. The same method was used to simulate the optimized HAART regimen. 
In all simulations and if the subject's uninfected $\mathrm{CD}^{+} \mathrm{T}$-cell count did not call for the initiation of treatment after two simulated years, the simulation of that subject was terminated with an output that treatment was not needed. The range of successful treatment coverage (ROC), defined as the ability of the treatment to suppress the HIV titer below 50 copies $/ \mathrm{mL}$ by the end of the 48 -week treatment cycle, was calculated for each test case. The results were compared to the ROC calculated for each cohort using the two optimized HAART regimens.

\subsection{Results}

\subsubsection{Optimal HAART Dosage for Base Case}

Optimization of the HAART regimen for the average subject was based upon the objective function, described in Methods, where total HAART dosage over the 48-week treatment period. The resulting predicted treatment strategy is shown in Figure 4.2. Full dosage $(100 \%)$ was prescribed for the first 31 days of the treatment regimen. The concentration of HAART prescribed then rapidly decreased, with dosages between $70 \%$ and $78 \%$ of full dosage prescribed between day 42 and day 324 . HAART was prescribed at a concentration of $68.8 \%$ of full dosage for the last 10 days of the regimen. The total amount of HAART prescribed over the 48 weeks was $78.1 \%$ of maximum HAART dosage. 


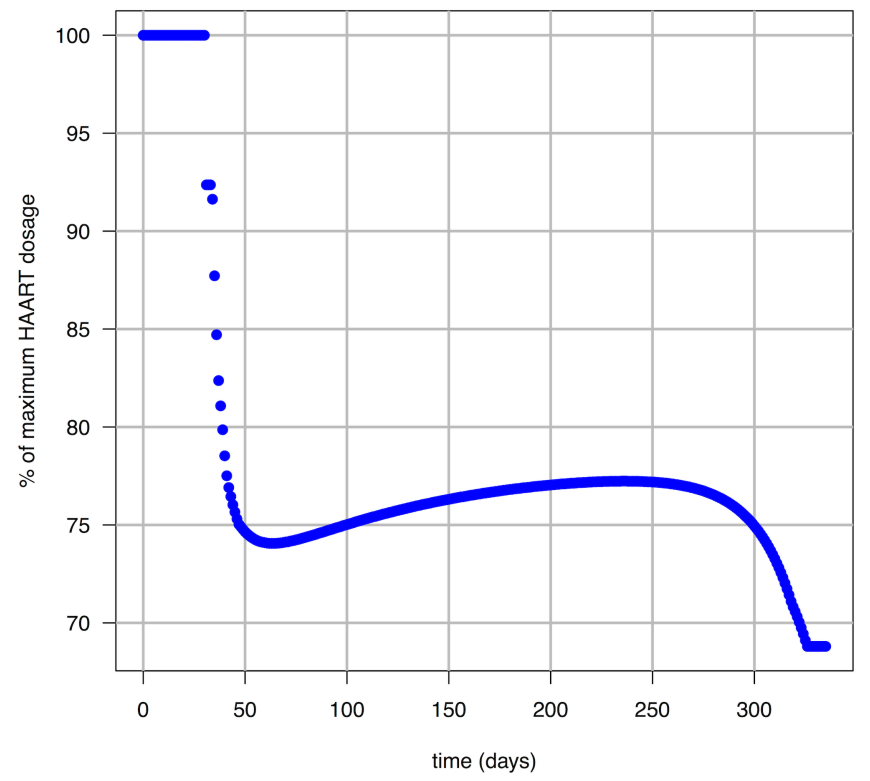

Figure 4.2. HAART Treatment Strategy Optimized for the Average Subject. Daily dosages of HAART are plotted over 48 weeks (336 days) as percentages of the maximum HAART dosage. HAART was optimized to minimize the total HAART dosage administered subject to the constraint that the subject's viral load remains below 50 virons/mL. Total HAART dosage over the 48 -week period prescribed by the optimized treatment regimen is $78.1 \%$ of the total possible HAART dosage.

\subsubsection{Comparison of HAART Strategies via Simulation Studies}

The range of successful treatment coverage (ROC) was calculated for multiple simulated cohorts of subjects receiving simulated standard treatment, optimized treatment, 1w/1w STI, and $8 \mathrm{w} / 2 \mathrm{w}$ STI. Successful treatment in this modeling was defined as the ability of the treatment to suppress the HIV titer below 50 copies $/ \mathrm{mL}$ at the 48-week time point. Table 4.1 lists the total amount of HAART administered by each treatment regimen.

The ROC that resulted by modeling the standard HAART dosage in ten in silico cohorts of 1,000 in silico subjects decreased from $100 \%$ to $75.15 \% \pm 1.68 \%$ as the 
variation in HIV model parameters increased, as shown in Figure 4.3. When optimized HAART dosing was used to treat each computer-generated cohort, ROCs generally decreased as variation in HIV model parameters increased. $8 \mathrm{w} / 2 \mathrm{w}$ STI ROCs decreased as variation in HIV model parameters increased also. Conversely, ROCs observed for the 1w/1w STI increased as the variation in HIV model parameters increased.

The different treatment regimens delineated different patterns of coverage as simulated inter-patient variation increased. For example, $1 \mathrm{w} / 1 \mathrm{w}$ STI showed a pattern of increasing ROC with increasing variability among subjects, while conversely, optimized HAART, standard HAART, and 8w/2w STI showed a pattern of decreasing ROC. Also, the generation of "extreme cases" or computer-generated subjects with the ability to be strongly resistant or highly susceptible to infection was more likely to occur as interpatient variation was increased. As population variability increased, treatment strategies that provided smaller overall drug exposure, such as the $1 \mathrm{w} / 1 \mathrm{w}$ STI, were more successful due to the increased number of individuals better able to deal with the disease. Treatment strategies that uniformly provided higher overall drug exposures, such as standard HAART, had lower ROCs in cohorts of greater inter-patient variability. The cause of the lower ROCs was because in silico subjects who were highly susceptible to infection were more likely to be generated, and as a result even the higher doses of HAART were not able to control their infection. ROCs observed for optimized HAART, which had the tendency to more precisely dose subjects, were found to respond much like standard treatment to increases in inter-patient variation. 


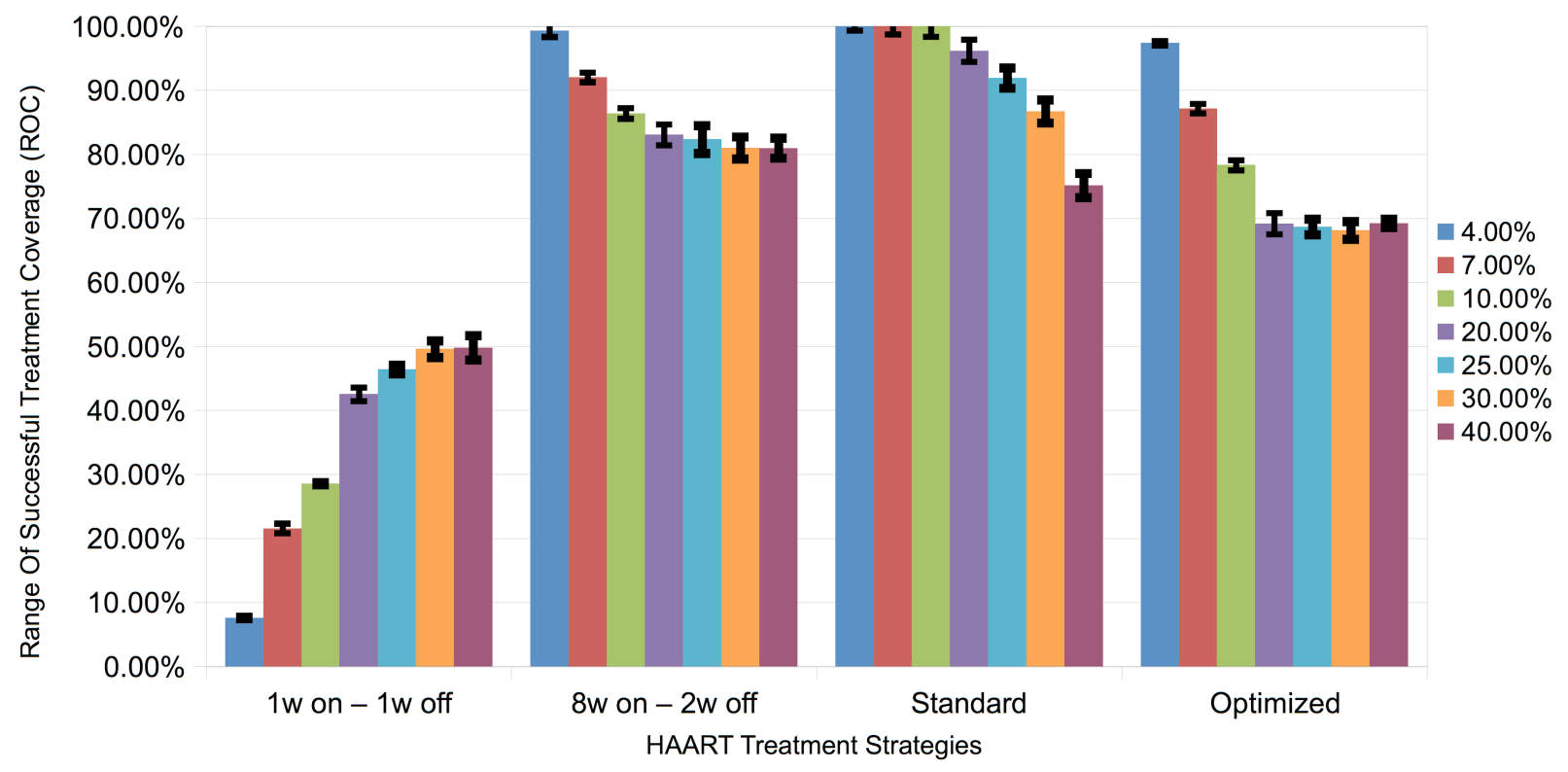

Figure 4.3. HAART Range of Coverage for in silico Cohorts Using Standard and Customized Treatment. The range of coverage, or percentage of subjects successfully responding to HAART, is plotted using each treatment strategy under investigation. HAART was applied to in silico cohorts simulating variation among cohorts by varying the HIV dynamic model parameters by $4 \%, 7 \%, 10 \%, 20 \%, 25 \%, 30 \%$, and $40 \%$. Successful treatment was regarded as the ability of the treatment strategy to suppress a subject's HIV titer below 50 copies $/ \mathrm{mL}$ after 48 weeks.

\subsubsection{Comparison of Simulation to Experimental STI Results}

The ROCs observed by simulating inter-patient variation at $25 \%$ variability in the model parameters were found to be most comparable to ROCs observed clinically, as shown in Figure 4.4. Significant discrepancies in clinical ROCs and ROCs predicted using the other measures of inter-patient variation were observed (data not shown). The clinical range of successful treatment coverage for standard treatment, defined as those 
subjects whose HIV titer were undetectable after 48 weeks of treatment (Ledergerber et al. 1999) was approximately $90 \%$.

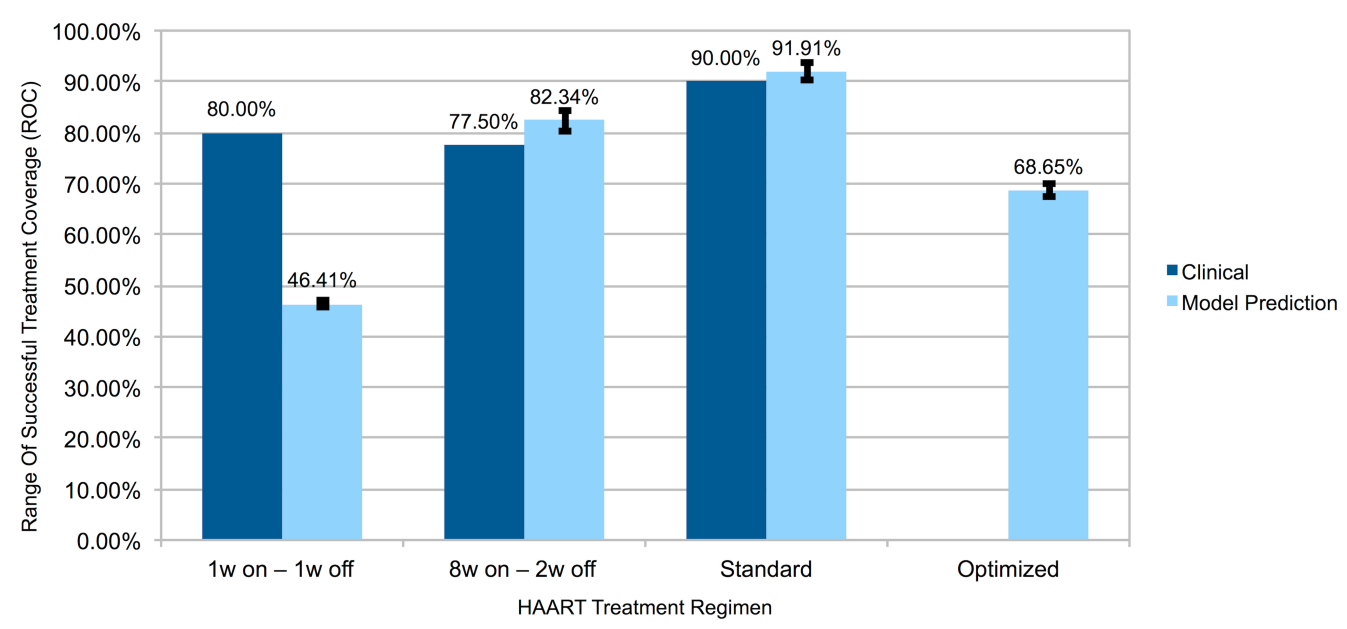

Figure 4.4. Comparison of Clinical Range of Coverage (ROC) to in silico ROC. The clinical (ROC), or percentage of subjects successfully responding to HAART, pertaining to 2 standard treatment interruption (STI) studies (Dybul et al. 2001, Fagard et al. 2003) and standard treatment (Ledergerber et al. 1999) was compared to in silico ROCs predicted by this study. The best results were observed when inter-patient variation was simulated by $25 \%$ variation in the HIV model parameters. The in silico ROC describing the success of optimized HAART was found be greater than that of 1 week on -1 week off treatment and less than that of full and 8 weeks on - 2 weeks off STI.

The $8 \mathrm{w} / 2 \mathrm{w}$ STI was predicted by our study to provide a high ROC. In the clinical study, subjects were required to have an undetectable viral load ([HIV] $<50$ copies $/ \mathrm{mL}$ for 6 months prior to study) and a CD4 count of 300,000 cells $/ \mathrm{mL}$ in order to participate in the trials (Fagard et al. 2003). In the published clinical results, this strategy provided a $77.5 \%$ ROC after 40 weeks of treatment. For this STI strategy, using a simulated inter- 
patient variation of $25 \%$ in the model parameters, the ROC predicted by the simulation $(82.34 \% \pm 1.22 \%)$ was very close to the ROC found clinically $(77.5 \%)$.

The $1 \mathrm{w} / 1 \mathrm{w}$ STI strategy was predicted to provide the lowest ROC. In the clinical study carried out by Dybul et al (Dybul et al. 2001), ten subjects were enrolled that had previously responded well to continuous treatment and entered with HIV RNA levels below 50 copies $/ \mathrm{mL}$ and $\mathrm{CD}^{+}$T-cell counts above 300,000 cells $/ \mathrm{mL}$. Eight of the ten subjects who completed this study were observed to maintain an HIV titer below 50 copies $/ \mathrm{mL}$. It was also observed that small changes in adherence that increased the length of the STI by a few days would lead to loss of control of the HIV titer. The ROC calculated for the $1 \mathrm{w} / 1 \mathrm{w}$ STI in the in silico cohorts was $46.41 \% \pm 1.59 \%$ at a simulated inter-patient variation of $25 \%$ in the HIV model parameters. The estimated ROC was considerably lower than observed in the clinical proof-of-concept study (Dybul et al. 2001). This result could be explained by the fact that the patients in the clinical study were described as those who had long-term control of plasma viremia and potentially had not developed drug resistance during suboptimal antiviral therapy. As a result, these individuals likely represented a subset of the in silico population with more robust immune parameter values.

\subsection{Discussion}

Administration of standard HAART yielded the best range of successful treatment coverage in each cohort while $1 \mathrm{w} / 1 \mathrm{w}$ STI yielded a smaller range of coverage as has been observed clinically. The $8 \mathrm{w} / 2 \mathrm{w}$ STI treatment strategy provided a range of coverage 
approximating that of full HAART despite administration of $16.7 \%$ less drug than the maximum; this finding was consistent with published clinical trial results in vivo.

Our study then evaluated if even lower levels of drug exposure could be optimized in a computer-generated in silico population to retain suppression of HIV-1 viremia. A theoretical HAART regimen was optimized to minimize total HAART administered for the average subject with the constraint that HIV-1 titers were to be reduced below detectable limits. It was predicted to successfully treat almost $70 \%$ of a computer-generated cohort while using a smaller amount of drug then the standard HAART strategy or the $8 \mathrm{w} / 2 \mathrm{w}$ strategy. Although ROCs yielded by optimized HAART in the model were somewhat lower than those from maximum HAART dosage administered under standard therapy, lower drug exposure prescribed by optimized HAART implies less severe detrimental side effects. As a result, the potential exists for improving the quality of life for a significant number of infected individuals. The range of coverage results predicted by the in silico model demonstrate the ability of a systems biology approach to optimize HAART for a theoretical subject as well as to predict the effectiveness of a HAART treatment strategy for a theoretical cohort in advance of exposing real human subjects to the hazards of a clinical trial. For example, the model correctly predicted the limited ROC for most of the $1 \mathrm{w} / 1 \mathrm{w}$ STI. The poor response to this STI strategy as demonstrated in the modeling confirmed the results of the clinical trials (Dybul et al. 2001). Thus, the approach utilized in this study has the potential to save the significant time and resources expended on such clinical trials and avoid patient risk. More importantly, such modeling could theoretically prevent patients from being put at unnecessary risk during testing of therapeutic strategies unlikely to be effective. The 
relative ease and low-cost of testing novel treatment strategies with a systems biology approach can potentially predict clinical outcomes in a large in silico cohort without the time and resources required for completion of a clinical trial. As a result, the approach described in this study may facilitate hypothesis generation and provide preliminary exploratory analysis of new therapies or treatment strategies. It may furthermore assist in the development of more focused and precise treatment improving the likelihood of success and minimizing the likelihood of failure.

Our study also shows that "one-size-fits-all" treatment strategies fail to strike an adequate balance between viral control and minimization of drug dosage. The standard HAART regimen and the $8 \mathrm{w} / 2 \mathrm{w}$ STI regimen prescribe more HAART and yield greater ROCs. Even though these two treatment regimens have high ROCs, they also cause increased risk of the treatment-associated side effects previously described. It is thus of interest to develop HAART treatment strategies which balance the need to administer a high enough concentration of HAART to control the infection while also administering a small enough concentration of HAART to minimize risk of treatment-associated side effects. This treatment strategy is consistent with modeling directed to an optimal treatment strategy.

HAART in the current study was optimized to the average subject described by a published model of HIV infection using an objective function calling for the minimization of HAART dosage while maintaining the HIV titer below 50 copies $/ \mathrm{mL}$. The same method of HAART optimization for the average subject could potentially be applied to derive an in silico model using an individual subject's CD4+ T-cell counts and HIV-1 titers so as to optimize HAART for that individual subject. The modeling could 
theoretically be employed using data on an individual's immune and viral kinetics to optimize dosing for that particular individual. Appropriate modeling could identify those patients who either require more than average antiretroviral drug exposure to maintain virologic suppression, or those that could be safely provided lower levels of antiretroviral drug exposure to reduce the incidence of medication-related adverse effects while still maintaining durable virologic suppression. Patients identified at the extremes of variability in the models could be candidates for individually modified and optimized HAART strategies. As personalized medicine becomes more predominant, HAART dosing that is customized to each individual patient based on individual kinetics will likely be an important strategy to achieve maximal therapeutic efficacy with minimal exposure to drug.

This study demonstrates the theoretic application of differing HAART strategies by analyzing computer-generated in silico cohorts of subjects with a systems biology approach. Such an approach can potentially predict greater treatment optimization and reduced antiretroviral drug exposures. These systems biology methods analyzing HIV-1 treatment optimization and range of coverage prediction have significant implications for the evolving field of personalized medicine. Using optimization of a daily HAART regimen for treatment of HIV-1 in a computer-generated individual considered to be the average individual as a model system, it was shown that the range of successful treatment coverage of optimized HAART is much greater than that of most experimental STI regimens. As a result there is considerable potential for systems biology to play an integral role in optimizing the therapy of HIV-1 patients that administers the minimum HAART dosage while still maintaining durable viral suppression. In addition, there are 
implications for the emerging field of personalized medicine. Specifically, the method presented for HAART optimization may be applied to the individual patient based on their specific physiological profile as characterized by their immune response kinetics. 
CHAPTER 5

DISCOVERY OF BIOCHEMICAL KINETICS AND MECHANISM USING A GEP-BASED EVOLUTIONARY ALGORITHM 


\subsection{Introduction}

Mathematical models are used in nearly all industries to analyze data, make predictions, and propose improvements based upon those predictions. Mechanistic models describing chemical kinetic data sets are often defined by making assumptions as to some model structure. Modeling of viral systems such as HIV-1, hepatitis B, and others has been of great interest in the hopes of developing treatment strategies based upon those models (Aviran et al. 2010, Bonhoeffer et al. 1997(a,b), Burg et al. 2009, de Sousa and Cunha 2010, Guedj and Neumann 2010, Herz et al. 1996, Krishnan and Dixit 2011, Nowak and May 2000, Perelson 2002, Prosperi et al. 2009, Ribeiro and Bonhoeffer 2000, Srivastava et al. 2002, von Kleist et al. 2010, Wu and Zhang 2010, Zeng and Yang 2010) . Many models of various sizes and structures have been proposed for these systems taking into account a wide range of biochemical species. Depending on the species taken into account and their predicted relationships, the model behavior can vary in response to any type of perturbation. The most comprehensive approach for determining models describing biochemical systems would be to assume no prior knowledge about the systems and the way that the species interact. In theory, it should be possible to leverage an evolutionary algorithm to infer a mechanistic model describing the species and their interactions, including some that may not be inherently obvious. The resulting model could then be used to make predictions about the system, as well as to test and optimize potential therapeutics.

Recently, work has been done on model generation from experimental data sets without assuming any prior knowledge about system mechanism. Schmidt et al.'s Eureqa formulize, a symbolic regression package that uses a genetic programming approach, 
quickly fits models to input data (Schmidt and Lipson 2009). Although these models provide a good fit to experimental data, they often do not provide mechanistic information as to how species in a biological system interact. Chattopadhyay et al. developed an algorithm for inferring stochastic reaction mechanism from experimental data (Chattopadhyay et al. 2013). This algorithm was successful at generating correct mechanistic models on the algorithm's error-complexity Pareto front, but some knowledge of the system was required in order to select the correct model from a set of potential models. A study by Bazil et al. provided a method for the reverse engineering of biological networks by generating systems of ordinary differential equations from experimental data (Bazil et al. 2011). This algorithm was successful in identifying candidate pathways that were known to exist but also generated many false positive pathways, as the algorithm was designed to minimize the number of false negative pathways identified.

A common method used in these approaches is that of genetic programming. Genetic programming is a type of evolutionary algorithm that generates programs or models of varying size and structure while not assuming any pre-defined model structure. The model is chosen by defining a set of functions (i.e. $+,-,{ }^{*}, /$ ), and a set of terminals (i.e. variables, real numbers) as building blocks from which to construct equations (Koza 1992). Genetic programming works by generating a population of potential models of a problem where each solution consists of the defined functions and terminals. The potential models are ranked or scored based upon a defined fitness function. Models are then selected, usually in proportion to their fitness score, to undergo recombination with other models. The models may also undergo mutation to further increase population 
diversity. New models are incorporated with selected existing models, and the new population of models is carried on to the next iteration where the algorithm repeats itself. A few examples where genetic programming has been used to create models addressing many different problems include the evolution of natural laws (Schmidt and Lipson 2009, Iba 2008), computer vulnerability testing (Kayacik et al. 2011), prediction of longitudinal dispersion coefficients in streams (Azamathulla and Ghani 2011), protein binding sites (Bains et al. 2004), interpretation of microbial flow cytometric data (Davey and Davey 2011), embedding and decoding of digital watermarks (Usman et al. 2011), synthesis of polymorphic combinational circuits (Gajda and Sekanina 2011), pipe break prediction modeling (Xu et al. 2011), estimation of daily pan evaporation (Shiri and Kisi 2011), software engineering predictive modeling (Afzal and Torkar 2011), modular neural network programming (Tsai and Lin 2011), self-reproducing machines (Zykov et al. 2005), and steel beam load capacity prediction models (Gandomi et al. 2011).

Models evaluated using genetic programming are described by parse trees, as shown in Figure 5.1. When randomly constructing a model, a node of the parse tree can be chosen to be a function from the defined function set or a terminal from the defined terminal set. When a function is chosen, two additional nodes in this case are generated to be evaluated by that function. The selection of a terminal will terminate a branch of the parse tree.

The parse tree structure has led to problems associated with genetic programming. The problem of "bloat" refers to the expansion of parse trees to very large depths (Iba 2008, Koza 1992). Bloat is a result of over-fitting a data set. The bloat problem has been addressed by defining the maximum depth that a tree can reach or by using a scoring 
function that penalizes larger solutions. Either solution to the bloat problem comes with a cost. Using the maximum depth approach, a function that checks the depth of each newly generated solution is called during each iteration of the algorithm, thus increasing the computational cost of the process. When using a scoring function that penalizes larger solutions, smaller programs that may not account for the complexity of the system tend to dominate the population of models. Therefore, an optimal solution of moderate to large depth that potentially better captures system complexity will have a smaller chance to be evolved within the population of solutions.

Secondly, the parse tree structure leads to a highly discontinuous search space over which the algorithm must find for the optimal model. Using the example equation presented in Figure 5.1, the model as defined would asymptotically approach a steady state after some period of time. If the "“" node was changed to a "+" node by a point mutation, the model would no longer converge to a steady state. Instead, the model would increase without bound, resulting in a poor fitness score and likely removal from the population of solutions. The result is a convergence problem because the tree with the "+" node is only 1 point mutation away from the optimal solution and therefore would be desirable to keep in the population of solutions. As larger systems are explored, this problem is exacerbated. Therefore, the phenotypic search space over which the algorithm must explore is highly discontinuous as changing one node or terminal, analogous to moving to a neighboring point in the search space, can yield a major change in the behavior and therefore the score of the solution. 


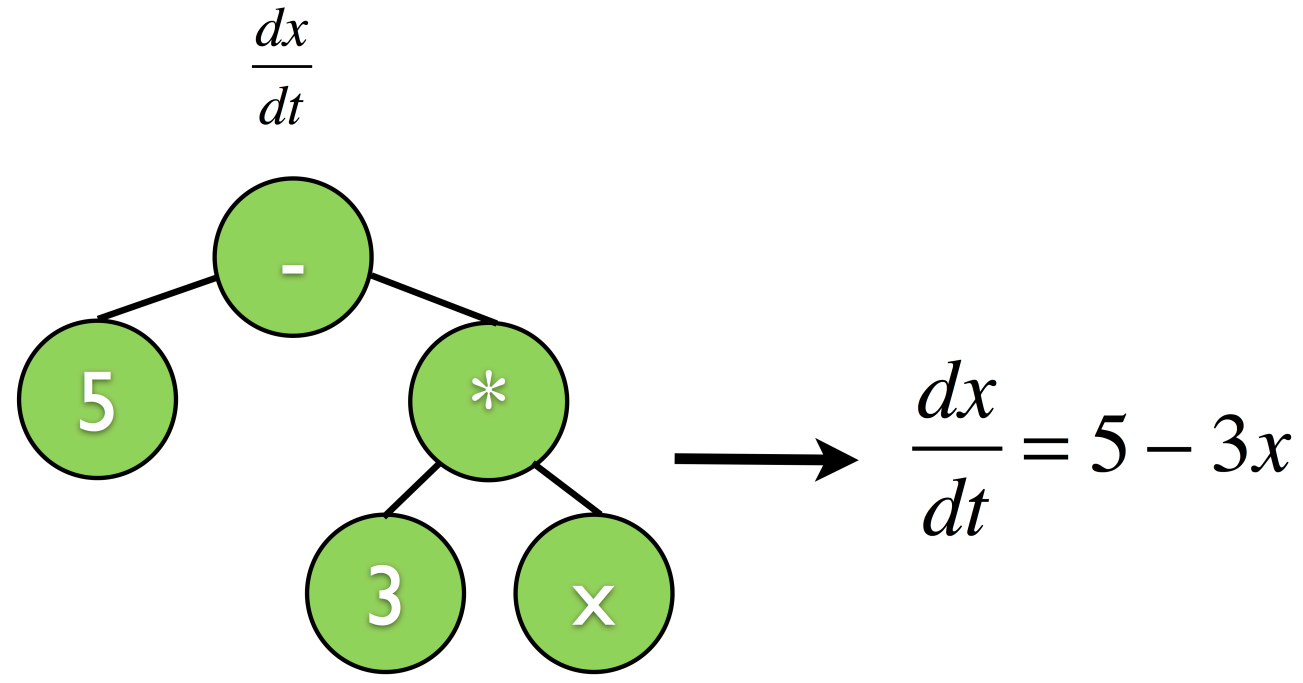

Figure 5.1. Parse-tree solution structure for genetic programming. This one-equation system is represented as a mass balance describing the change in some species $x$ over time. Such an equation could be generated using a genetic programming algorithm. Each node can be selected to be one of a set of functions or one of a set of terminals, where each function requires two arguments.

Gene expression programming (GEP) has been proposed to help address these problems by encoding the different sizes and shapes of parse trees in linear solutions of fixed length (Ferreira 2006). These linear solutions are then mapped to parse trees that can be simulated. This approach mimics the genotype to phenotype mapping observed in nature. In this study, the GEP enhancement to genetic programming was tailored to the evolution of multi-variate systems describing reaction kinetic data to address the discontinuous search space observed for these types of problems.

Genetic programming and GEP both use three primary evolutionary operations: selection, recombination, and mutation. These operators are used to evoke changes in a population of solutions thereby exploring a search space and converging on an optimal 
solution. Selection has received some attention as a function that can be tailored to increase the efficiency and efficacy of a genetic programming algorithm (Gallagher and Frean 2005, Gero and Kazakov 2001, Rowe et al. 2004, White and Srivastava 2011, Whitley 1989). Varieties of selection operations that have been described include elite selection (Bautista et al. 2013, Costa and Oliveira 2003), tournament selection (Blickle and Lothar 1995), proportional selection (Schwefel 1993), and linear ranking selection (Baker 1985). Elite selection requires the copying of an elite fraction of the best scoring solution from one generation to the next. The rest of the next generation population of solutions is made up of randomly recombined and mutated solutions. Tournament and proportional selection bias the selection of solutions for recombination and mutation by their score as determined by the scoring function. Linear ranking selection biases the selection of solutions for recombination and mutation based upon a solutions rank within the population as opposed to its score. Application of the appropriate selection operation has the potential to increase efficiency and therefore feasibility of a GEP/genetic programming approach.

It was hypothesized that by leveraging knowledge of reaction kinetic theory, mechanistic mathematical models of complex biological interactions could be inferred using an evolutionary algorithm. To test this hypothesis, an evolutionary algorithm that optimized reaction kinetic models of biological systems from time series data sets was developed. The traditional genetic programming methodology was enhanced to evolve more complex reaction kinetic models describing multi-variate data sets using a GEPbased approach. The performance of this algorithm was evaluated by its ability to recapitulate a generic viral dynamics model from a noisy in silico data set. 


\subsection{System and Methods}

In this proof-of-principle study, an evolutionary algorithm was used to rediscover the mechanism described by a model of viral dynamics (Srivastava et al. 2002) using computer-generated (in silico) data describing the system. The model, shown in Equations 5.1 - 5.3, describes the rate of change of viral template (tem), viral genome (gen), and viral structural proteins (struct) over time using six parameters. Figure 5.2 depicts a network diagram describing this system.

$$
\begin{gathered}
\frac{d[\text { tem }]}{d t}=k_{1}[\text { gen }]-k_{2}[\text { tem }] \\
\frac{d[\text { gen }]}{d t}=k_{3}[\text { tem }]-k_{2}[\text { gen }]-k_{4}[\text { gen }][\text { struct }] \\
\frac{d[\text { struct }]}{d t}=k_{5}[\text { tem }]-k_{6}[\text { struct }]-k_{4}[\text { gen }][\text { struct }]
\end{gathered}
$$




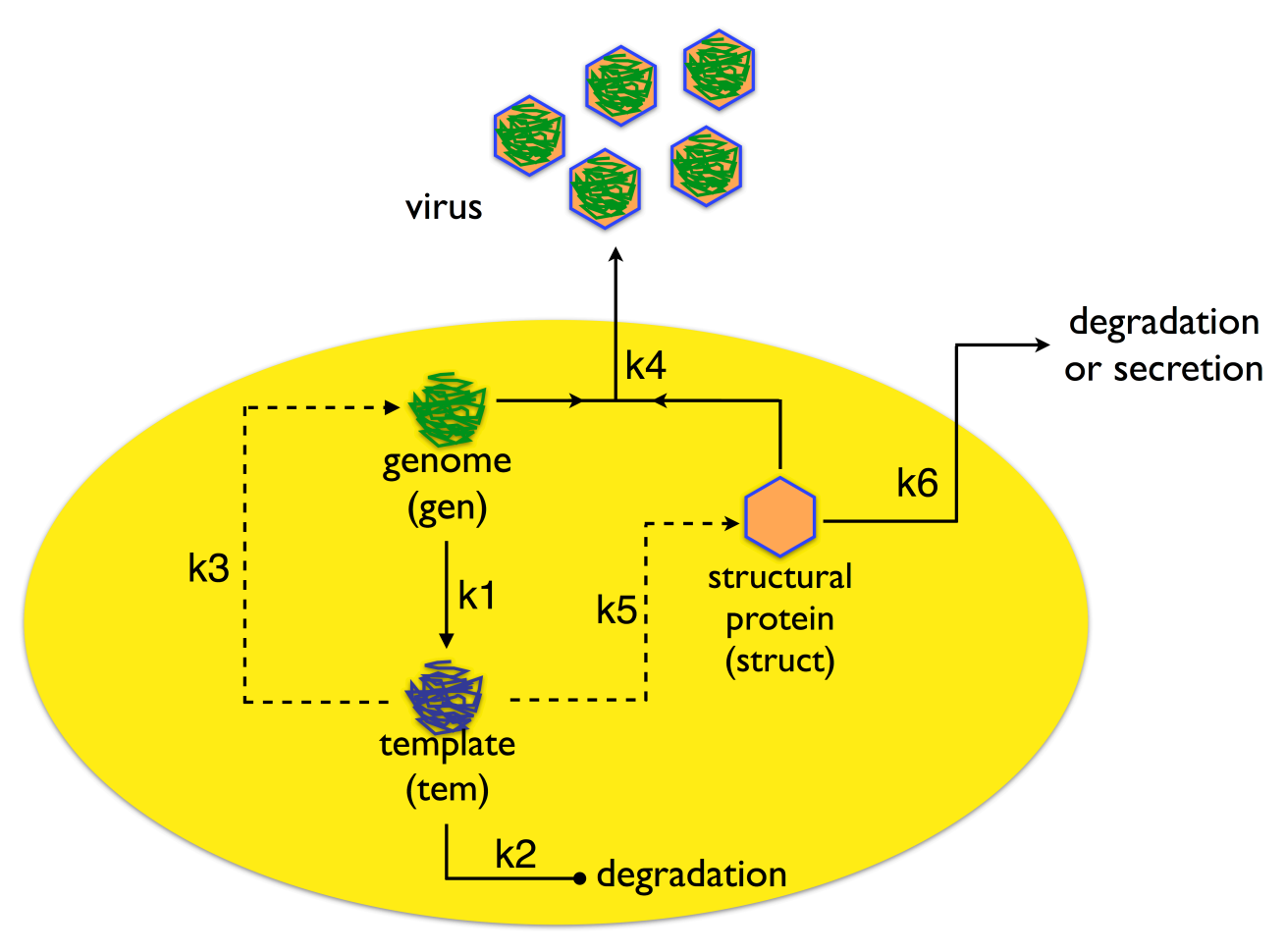

Figure 5.2. General viral dynamics system (Srivastava et al. 2002). The published model of general viral dynamics accounts for three species (tem, gen, and struct) participating in six interactions described by six parameters $\left(\mathrm{k}_{1} \rightarrow \mathrm{k}_{6}\right)$. Viral genome (gen) in the cytoplasm enters the nucleus to become viral template (tem). Tem catalytically up-regulates production of gen as well as structural proteins (struct). Both tem and struct can be degraded. Finally, struct can encapsulate gen to form new viral progeny that leave the system.

In this model, $\mathrm{k}_{1}$ describes the rate by which gen enters the nucleus and becomes tem. The model considers two degradation interactions, the degradation of tem at a rate of $\mathrm{k}_{2}$ and the degradation of struct at a rate of $\mathrm{k}_{6}$. Tem catalytically up-regulates gen at a rate of $\mathrm{k}_{3}$ and struct at a rate of $\mathrm{k}_{5}$. Finally, the interaction occurring at a rate of $\mathrm{k}_{4}$ models the encapsulation of gen by struct to yield new viral progeny that leaves the system. Table 5.1 lists the parameter values for this model (Srivastava et al. 2002). 


\begin{tabular}{|c|c|c|}
\hline \multicolumn{3}{|c|}{ Table 5.1. Viral Dynamics Model Parameters } \\
\hline Parameter & Value & Units \\
\hline $\mathrm{k}_{1}$ & 0.025 & day $^{-1}$ \\
\hline $\mathrm{k}_{2}$ & 0.25 & day $^{-1}$ \\
\hline $\mathrm{k}_{3}$ & 1.00 & day $^{-1}$ \\
\hline $\mathrm{k}_{4}$ & $7.5^{*} 10^{-8}$ & molecules $^{-1}$ day $^{-1}$ \\
\hline $\mathrm{k}_{5}$ & 1000 & day $^{-1}$ \\
\hline $\mathrm{k}_{6}$ & 1.99 & day $^{-1}$ \\
\hline
\end{tabular}

Genetic programming methods can represent programs or equations as trees made up of functions and terminals. In assembling random trees, some potential models may not include production or consumption terms for some of the mass balances. Potential models also might not include explicit parameters for some interactions (equivalent to an assumed reaction parameter of 1), and some terms may include tertiary and higher-order interactions. For the evolution of reaction kinetic models, a heuristic limiting the search space to models that include production and consumption terms for each species was applied. Each production and consumption interaction in each candidate model was also described by a reaction parameter. Finally, for the biological models of interest, it was assumed that each interaction could include one or two reactants that could catalyze or be consumed to generate one or two products (Levenspiel 1972). Tertiary and higher-order interactions were therefore eliminated from consideration.

To tailor a genetic programming approach to generate desired reaction kinetic models, the structure of candidate solutions was changed from a parse tree structure to the structure shown in Figure 5.3A. This representation, inspired by a GEP approach, encodes solutions as a "genotype," such as a list or collection of lists, which are then 
mapped to a "phenotype," such as a set of parse trees shown in Figure 5.3C. Therefore, this evolutionary algorithm was programmed to map a list of reactants, reaction parameters, products, and types of reactions to parse trees describing the time series derivative of each variable under consideration. Such an approach resulted in a reduction of the size of the search space over which the evolutionary algorithm navigated to find a mechanistic mathematical model. 
A

\begin{tabular}{|c|c|c|c|c|c|c|}
\hline & Int I & Int 2 & Int 3 & Int 4 & Int 5 & Int 6 \\
\hline Reactants & gen & tem & tem & $\begin{array}{c}\text { gen } \\
\text { struct }\end{array}$ & tem & struct \\
\hline $\begin{array}{c}\text { Rate Constant } \\
\text { \& Rxn Type }\end{array}$ & $\mathrm{k}_{1}, \mathrm{I}$ & $\mathrm{k}_{2}, \mathrm{I}$ & $\mathrm{k}_{3}, 0$ & $\mathrm{k}_{4}, \mathrm{I}$ & $\mathrm{k}_{5}, 0$ & $\mathrm{k}_{6}, \mathrm{I}$ \\
\hline Products & tem & deg & gen & deg & struct & deg \\
\hline
\end{tabular}

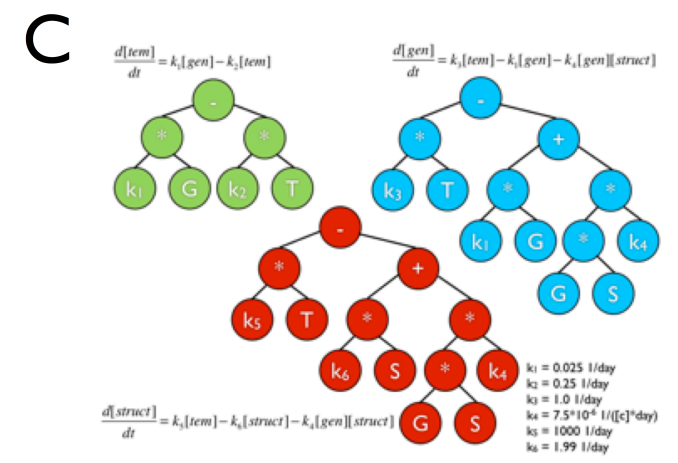

B
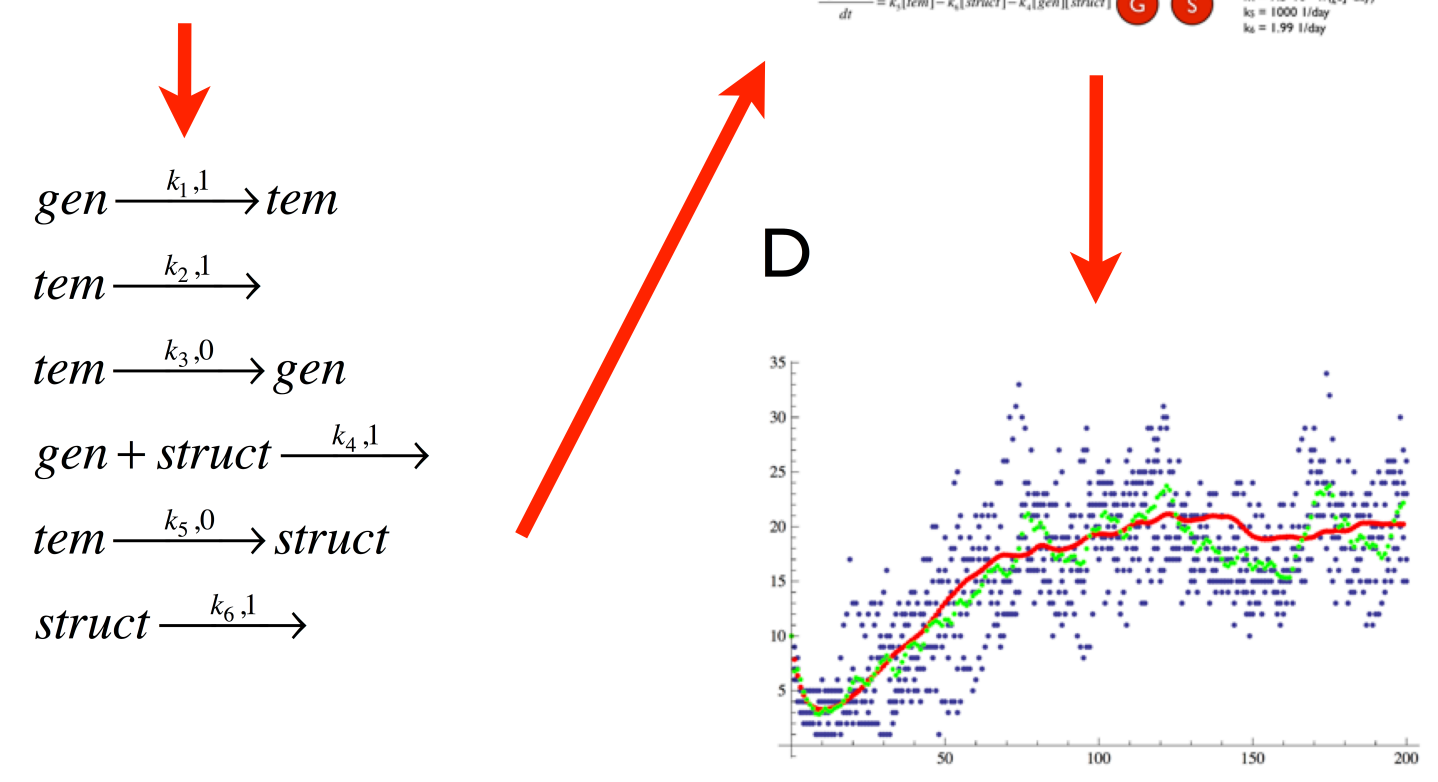

Figure 5.3. Mapping of model genotype to model phenotype in evolutionary algorithm. (A)

Each potential model was encoded as an interaction table describing reactants, reaction parameters, and products. Each interaction was also classified as a catalytic or consumption interaction. This table, representing a list of reactions (B), was then translated using mass action kinetic theory into a system of ordinary differential equations that could be represented as a set of parse trees (C). These equations were then simulated and scored against an experimental data set (D).

Another contributing factor to the large search space size the need to search for both the optimal model structure and optimal model parameters. Even if a good model structure was found, it was still unlikely that the algorithm would converge on the 
optimal set of parameters. To address this problem, parameter estimation was carried out for every potential model against the experimental data during each generation of the evolutionary algorithm.

\subsection{Algorithm}

It was observed in early versions of the algorithm that the absence of one necessary interaction could result in a poor scoring model that might not survive in the population of models (data not shown). This was yet another effect of the discontinuous search space associated with these types of problems. Such behavior goes against the assumption underlying evolutionary algorithms that optimal solutions could be built up gradually from suboptimal solutions containing pieces of the optimal solution. To resolve this issue, models were built up in a step-by-step fashion where only one equation was allowed to be modified at a time.

The biochemical kinetics discovery algorithm is first described for an n-species system. To generate a complete kinetic model, a mass balance for a single species was evolved. Simultaneously, the mass balances for the other species were described by fitted polynomials during scoring. Polynomials were fit to every three consecutive data points and stored for use in the algorithm. This combination of one evolved mass balance and n-1 polynomial fits was called an instance. One instance was generated for each species such that every species had an instance in which it was represented by an evolved mass balance equation. The general approach is outlined in Figure 5.4. The best scoring mass balance from the collection of instances was taken as the most probable result and that mass balance was fixed for the next step. The species described by this result was 
referred to as Species A. In other words, all of the remaining instances had their Species A polynomial fit replaced by the Species $A$ mass balance. During the next step, instances of the evolutionary algorithm were carried out to evolve a mass balance for each of the remaining species one at a time. The mass balance describing Species $A$ was fixed, and the mass balances describing the species not being evolved were described by a set of polynomials. Again, the best scoring result from this set of instances was fixed for the next step and this variable was defined as Species B. For a system of $n$-species, this procedure would continue until only one species remained for which a mass balance had not yet been evolved.

The evolutionary algorithm was then used to evolve a mass balance describing this last species, while the mass balances describing the other species were fixed. This species was defined as Species $\xi$ where $\xi$ related to the number of species for which a time series data set existed. After this, the mass balance for Species $A$ was re-evolved using the evolutionary algorithm where mass balances describing Species $B$ through $\xi$ were fixed. The existing result for Species $A$ and the newly evolved solution were compared by scoring with the current set of equations describing Species $B$ through $\xi$ and the best scoring solution was kept and fixed for subsequent steps. The mass balances for Species $B$ through $\xi$ were then re-evolved one at a time in the order that they were first evolved. This method was carried out cyclically until the mass balances describing Species $A$ through $\xi$ remained the same over one full cycle. The information gained from these steps was ultimately used to seed the first generation population of a final evolutionary step during which mass balances describing each of the species under investigation were simultaneously evolved. 


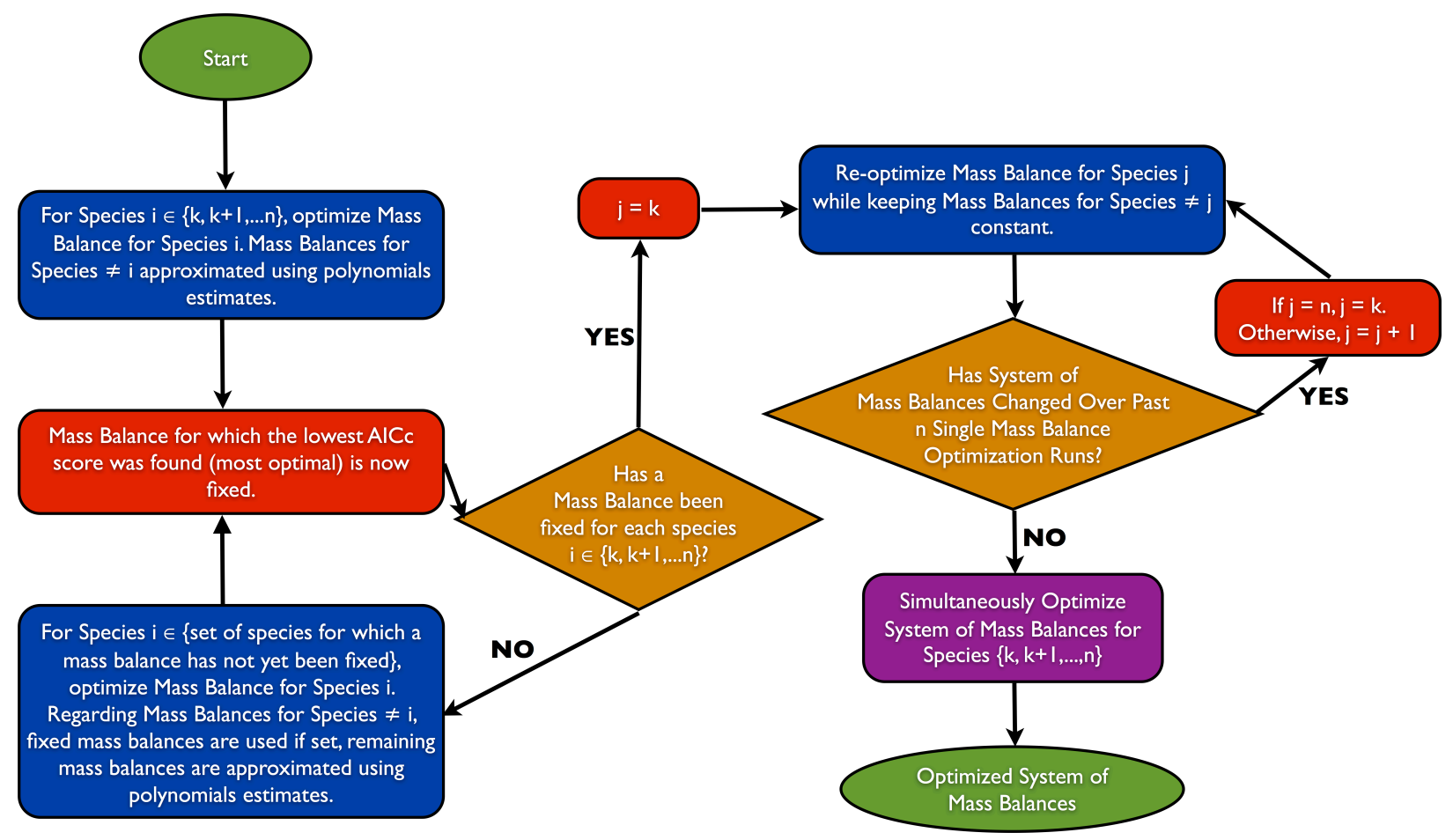

Figure 5.4. Algorithm for the discovery of a biochemical kinetic model describing an nvariable system. Mass balances were evolved in a step-by-step manner to reduce the search space during a single evolutionary algorithm instance. In the initial steps, species mass balances were approximated by a set of polynomials fit to the corresponding data set until a mass balance was evolved for that variable. Once a complete model was preliminarily proposed, each species' mass balance was re-evolved one at a time until the system of mass balances had not changed over one complete cycle. The model was finalized by carrying out one final evolutionary algorithm optimization during which all mass balances were simultaneously evolved. Figure 5.5 summarizes the procedure used during each step to evolve an optimized mass balance.

Inputs for this evolutionary algorithm included the maximum number of allowable interactions, the population size, the species under investigation, time series data sets describing these species, the initial conditions used to collect the data set, any fixed mass balances, and the number of generations to be run before the algorithm 
terminated. The number of interactions that potentially described a system increased exponentially with the number of species under investigation. Therefore the number of interactions allowed to describe a model was capped by the square of the number of species $\left(n^{2}\right)$ plus one in order to maintain computational tractability. $n^{2}$ represented the number of interactions required for each species to be up-regulated by each other species, to be consumed to produce each species, and to be degraded. Up to one additional interaction was considered as binary interactions and constitutive production interactions were also considered. Individual mass balances considered fewer interactions when evolved and each species had the chance to participate in every interaction during the simultaneous evolution step. 


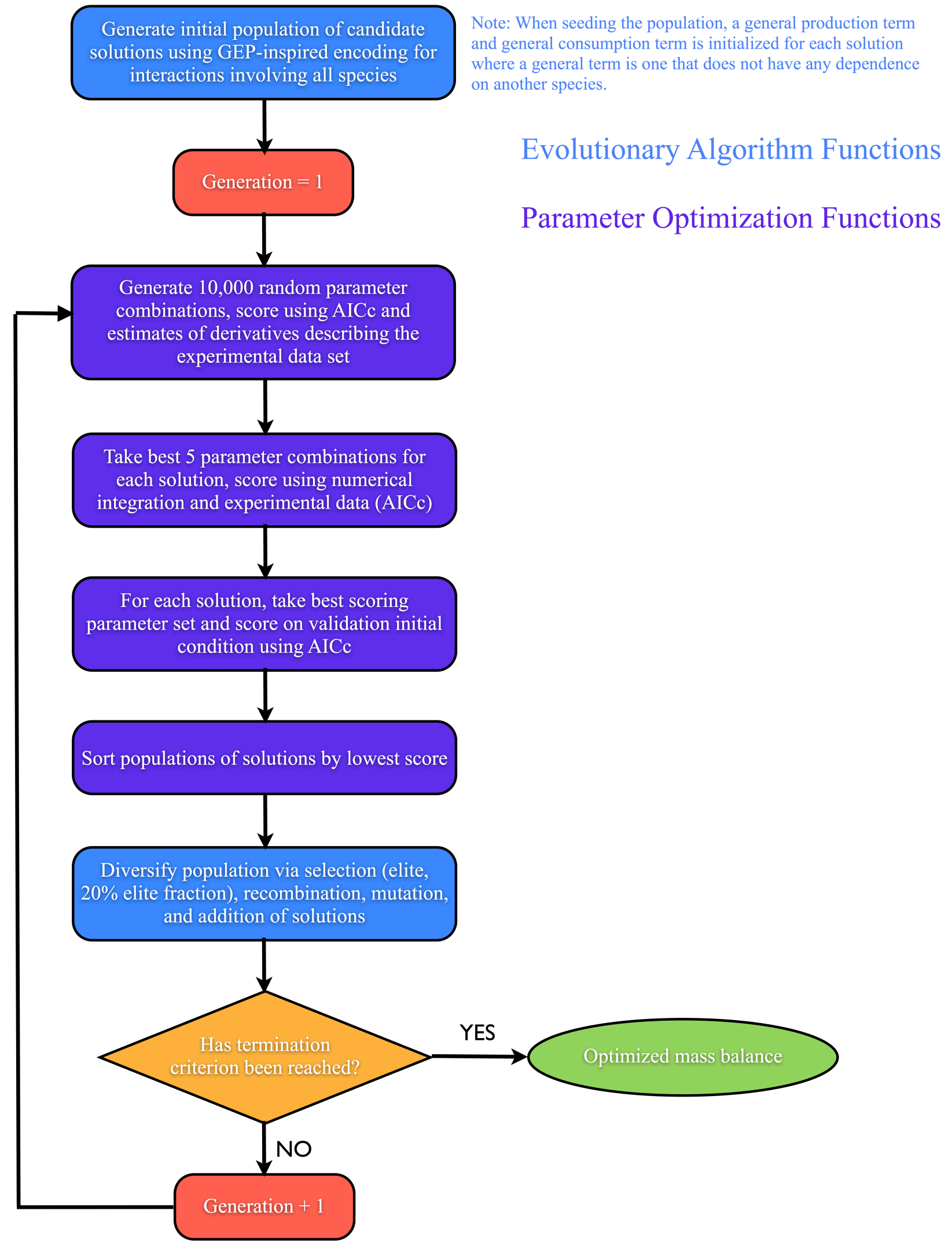


Figure 5.5. Evolutionary algorithm for the discovery of biochemical mechanistic model. This flowchart details the procedure for discovering a mechanistic model for an n-species system. An initial population of candidate solutions was generated as encoded interaction tables. During each generation of the algorithm, 10,000 random parameter combinations were generated and scored using model calculations of species accumulation and estimates of experimental data derivatives. The top five scoring parameter guesses were used as initial guesses to a numerical parameter optimization function with the exception of single mass balance steps where parameters were not numerically optimized until the final step. The best scoring parameter set was then used to score the solution against the validation condition. An elite selection strategy was applied. The best scoring solutions were copied to the next generation. Then solutions were selected, recombined, and mutated to fill out and diversify the next generation's population of solutions. This procedure was repeated until the termination criterion was reached. The top scoring solution in the final generation population was considered to be the optimized model.

Populations of potential solutions were generated and scored by simulating each model using Mathematica on each training condition. Each potential solution in the first generation population of individual mass balance runs was initialized with a constitutive production term and a first order degradation term. The simulation results were compared to the training data set and scored using the corrected Aikaike Information Criterion $\left(A_{1 C}\right)$ method (Aikaike 1974). Parameters were estimated for every solution every generation. During the simultaneous optimization step, 10,000 random parameter sets were generated and scores were estimated by comparing derivatives calculated using model mass balances to derivatives calculated using the polynomial fits to the experimental data set. The top five scoring parameter sets were then used as initial guesses for Mathematica's numerical optimization function. The objective function was 
minimization of the $\mathrm{AIC}_{\mathrm{c}}$ score. Once optimized, each potential solution with the best scoring parameter set was evaluated against a validation set of data using the $\mathrm{AIC}_{\mathrm{c}}$. This score was associated with the potential solution during subsequent evolutionary operations. During individual mass balance optimization steps, numerical integration was not used to optimize parameters. Instead the best of the parameter sets, as determined by $\mathrm{AIC}_{\mathrm{c}}$, was used for evaluation of the model on the validation data. The parameters for the best scoring mass balances at the end of multiple trials of the evolutionary algorithm were optimized using Mathematica and the best result was taken as the optimized solution.

Evolutionary algorithms utilize selection, recombination, and mutation operations to generate diversity in a population of solutions and facilitate convergence towards an optimized solution. An elite selection strategy was implemented where the top $20 \%$ of the best scoring solutions in the population were copied unaltered to the next generation population. The remaining $80 \%$ of the next generation population were made up of solutions randomly selected two at a time from the previous generation population that were given a chance to be recombined with each other and a chance to be mutated. In order to address potential premature convergence brought on by decreasing diversity of solutions in the population, the recombination rate, initiated at $90 \%$, was decreased by $10 \%$ every $\mathrm{x}$ generations, where $\mathrm{x}$ is equal to $10 \%$ of the number of generations required to terminate, until it reached $0 \%$. Conversely, the mutation rate, initiated at $5 \%$, was increased by $5 \%$ every $\mathrm{x}$ generations until it reached $50 \%$. In this way, solution mutation, which introduces new alleles into a population thereby increasing diversity in the population of solutions, was the favored evolutionary operation in later generations. 
This algorithm is computationally intensive, and its use may be limited by the large amount of time it would take to run for systems of many species. To address this issue, the operations that could be run in parallel, such as the simulation and scoring of candidate solutions, and the execution of multiple instances of the algorithm, were parallelized. The UCONN School of Engineering's high performance computing cluster consists of 768 Intel Xeon X5650 Westmere CPU cores over which parallel processes can be allocated. Mathematica's built-in parallel capabilities were used to carry out this task.

\subsection{Implementation}

The biochemical kinetics discovery algorithm was implemented to evolve the known mechanism of the generic viral dynamics system as a test case. An in silico data set was generated as the input to the algorithm. Data was generated from the published model using four conditions, listed in Table 5.2. Five independent samples were taken every four simulated days over a period of two hundred days using $10 \%$ noise generated over a normal distribution. During every step of the algorithm, a population of 30 potential solutions was evolved for up to 150 generations allowing for convergence of the population. During each individual mass balance optimization step, twenty-four trials of the evolutionary algorithm were carried out. The best scoring solution across all trials, including the best result from the previous instance, was taken as the new optimized mass balance. Likewise, eight trials of the evolutionary algorithm were carried out during the final simultaneous optimization step where the best scoring result was taken as the 
optimized model. A maximum of four interactions were allowed to evolve during single species steps and a cap of ten interactions was used for the combined evolution step.

\begin{tabular}{|l|c|c|c|}
\hline \multicolumn{4}{|c|}{ Table 5.2. Viral Dynamics Model Conditions For Data Generation } \\
\hline $\begin{array}{l}\text { Initial } \\
\text { Condition }\end{array}$ & {$[\text { tem }]_{0}$} & {$[\text { gen }]_{0}$} & {$[\text { struct }]_{0}$} \\
\hline 1 & 1500 & 0 & 0 \\
\hline 2 & 3000 & 0 & 0 \\
\hline 3 & 5000 & 0 & 0 \\
\hline 4 & 0 & 1500 & 0 \\
\hline
\end{tabular}

During the first three steps of the algorithm, a mass action kinetic equation was evolved first describing the change in $[$ tem $]$ over time, then the change in [gen] over time, and finally the change in $[$ struct $]$ over time. The interactions described by these mass balances are shown in Figure 5.6A. At this point in the evolution of the viral dynamics model, only one true pathway had been identified (the degradation of struct). 


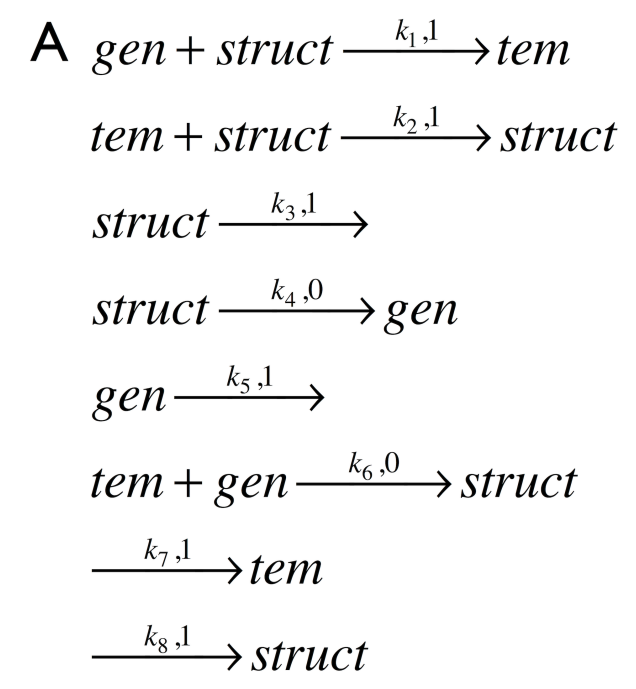

Model I

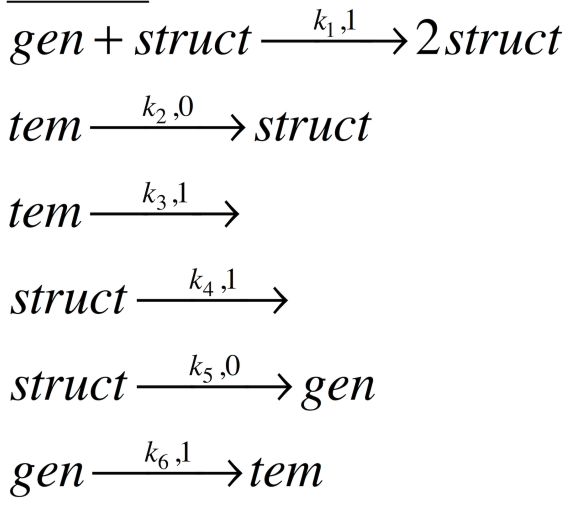

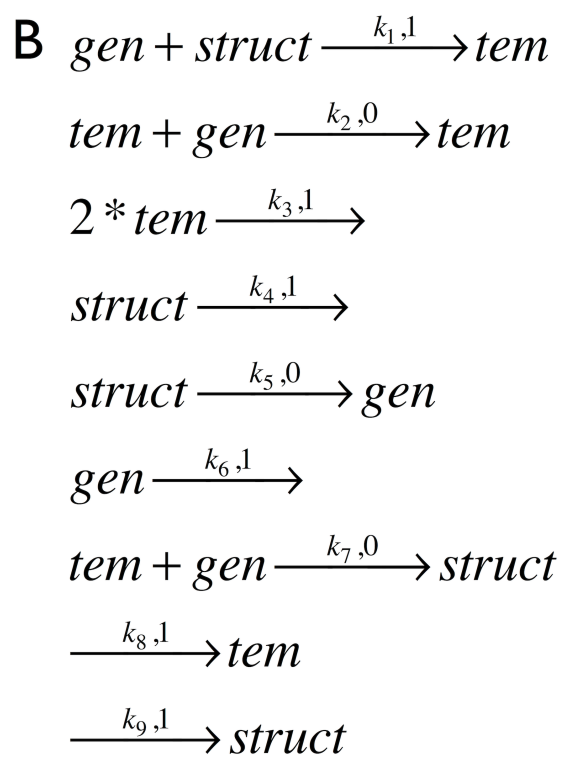

\section{Model 2}

gen + struct $\stackrel{k_{1}, 1}{\longrightarrow}$

tem $\stackrel{k_{2}, 0}{\longrightarrow}$ struct

tem $\stackrel{k_{3}, 1}{\longrightarrow}$

struct $\stackrel{k_{4}, 1}{\longrightarrow}$

tem $\stackrel{k_{5}, 0}{\longrightarrow}$ gen

gen $\stackrel{k_{6}, 1}{\longrightarrow}$ tem

Figure 5.6. Evolution of the general viral dynamics model. (A) Carrying out the first three steps of the algorithm (Figure 5.4) resulted in a complete model describing the three species involved in the viral dynamics system. This proposed model consisted of eight interactions, one of which was consistent with the published model. (B) After additional instances of single mass balance optimization, the algorithm converged upon a model structure that was used to seed the simultaneous optimization step. (C) After the step-by-step evolution of the model was complete, the mass balances for all three species were simultaneously evolved. The result was two identical scoring models, one of which evolved to the published general model of viral dynamics. Note: Type " 1 " reactions are standard reactions, and type " 0 " reactions are catalytic reactions. 
The solution shown in Figure 5.6B was used to seed the population for the last step during which all three mass balances were simultaneously re-evolved in order to determine the optimized model. The final two interactions in the seed model, shown in Figure $5.6 \mathrm{~B}$, describing the $0^{\text {th }}$-order production of tem and struct were eliminated to allow for more new interactions to enter the population. These two interactions were chosen for elimination because both tem and struct were up-regulated in other interactions and the constitutive production of tem and struct described by this zero-order reaction is very unlikely for this intracellular viral system. If these two interactions in fact were part of the system's network structure, they could have re-entered the model during the simultaneous optimization step. After 70 generations of the simultaneous optimization step, the algorithm converged on two solutions of equal score $\left(\mathrm{AIC}_{\mathrm{c}}=-\right.$ 149.9). These solutions are shown in Figure 5.6C as Model 1 and Model 2. Model 1 correctly predicted the degradation of tem and struct, the consumption of gen to yield tem, and the catalytic up-regulation of struct by tem. It incorrectly predicted that gen was catalytically up-regulated by struct and that gen and struct associated to yield a net of one struct molecule. Model 2 correctly predicted all six interactions of the generic viral dynamics model with no additional interactions. 


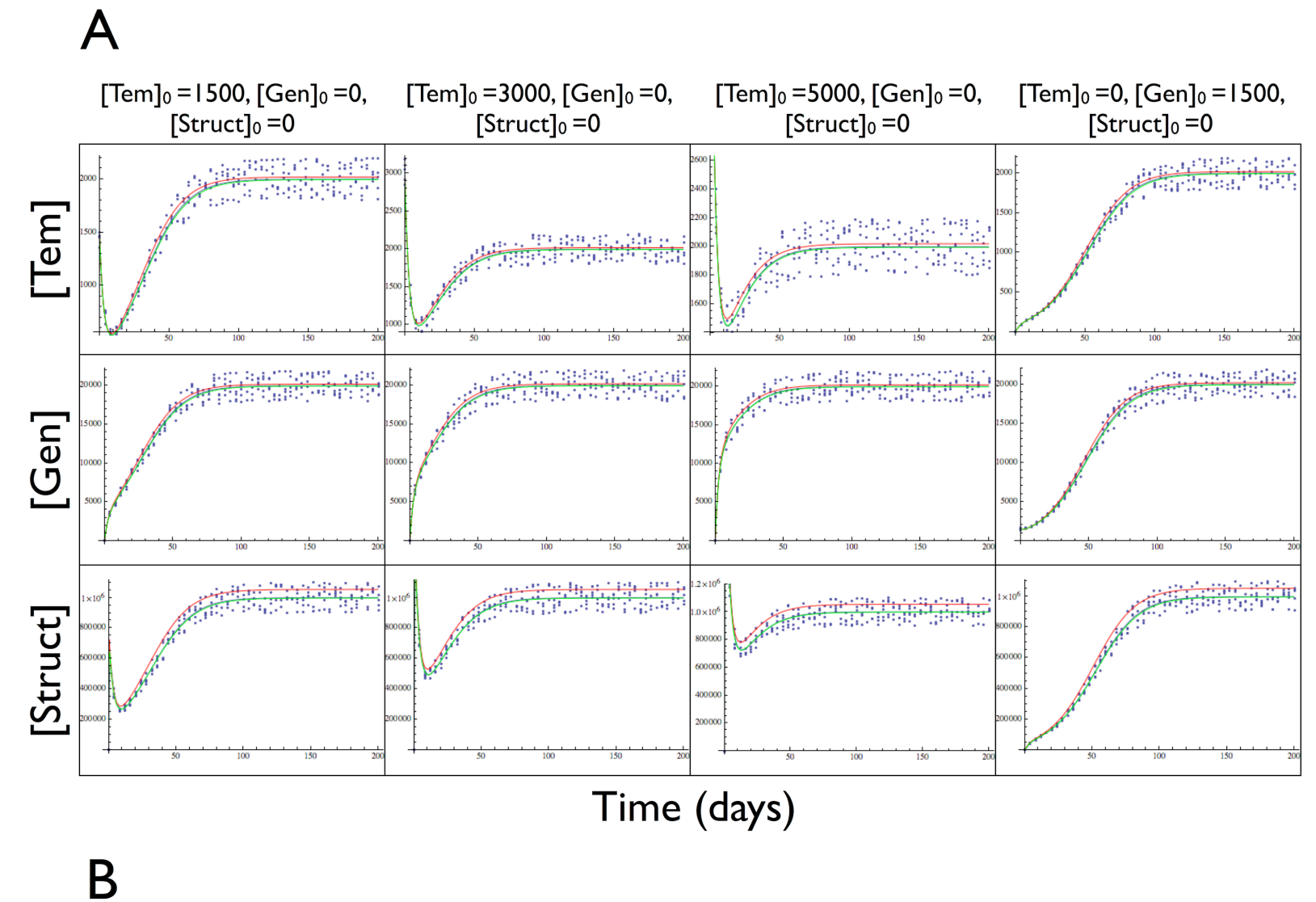

$[\text { Tem }]_{0}=10000,[\mathrm{Gen}]_{0}=0, \quad[\text { Tem }]_{0}=0,[\mathrm{Gen}]_{0}=5000, \quad[\text { Tem }]_{0}=500,[\text { Gen }]_{0}=5000, \quad[\text { Tem }]_{0}=0,[\mathrm{Gen}]_{0}=0$,

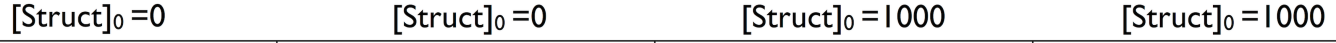

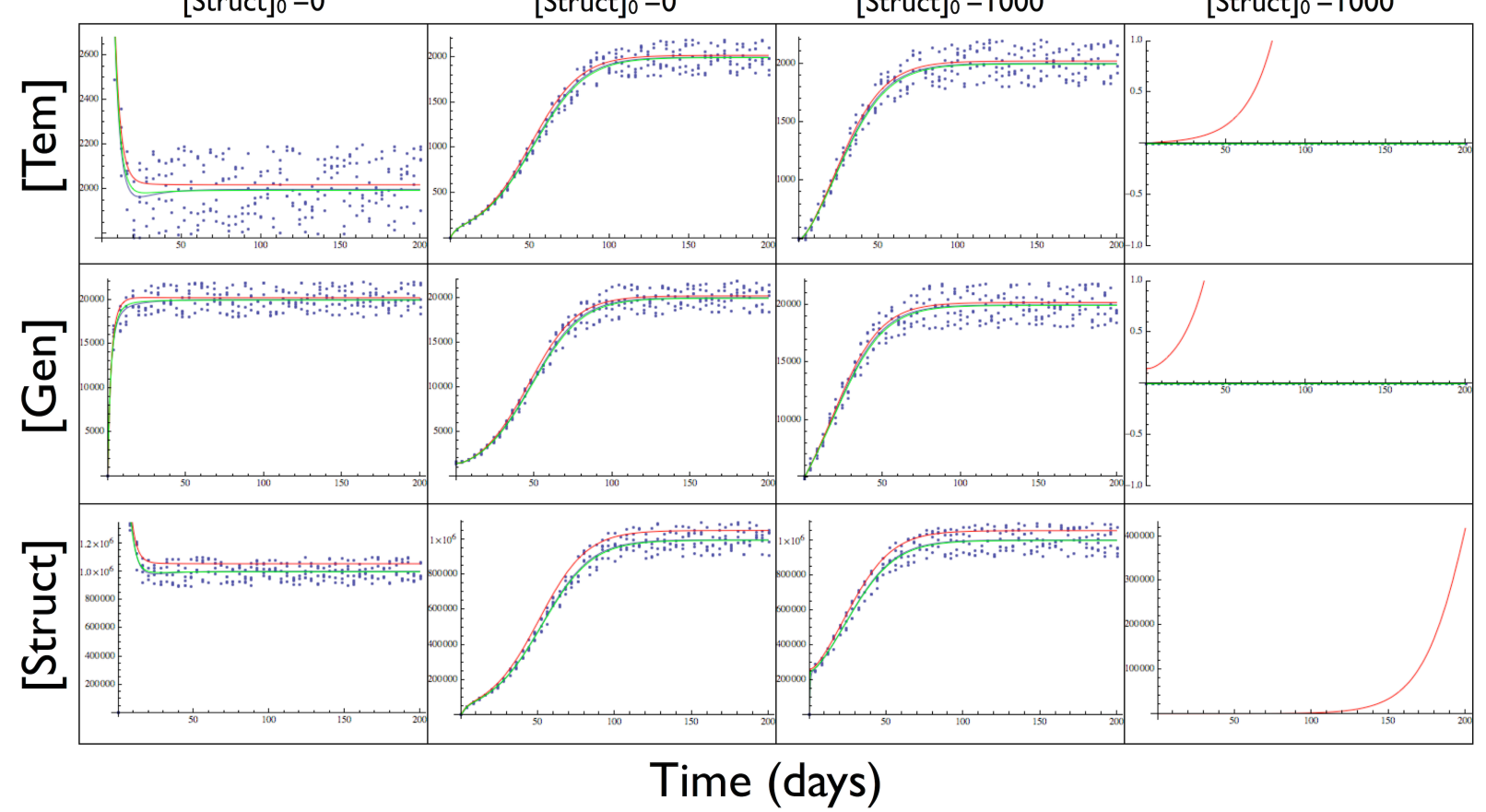

Color Legend: Blue $=$ in silico Data Set, Red $=$ Model 1, Green $=$ Model 2 
Figure 5.7. Simulation of evolved viral dynamics models. (A) Simulation of Models 1 and 2 against in silico data sets generated for the three training conditions (columns 1-3) and the validation condition (column 4) are shown. Both Models 1 and 2 had an $\mathrm{AIC}_{\mathrm{c}}$ score of -149.9 when scored against the validation condition. (B) Models 1 and 2 were tested by comparing simulation results to in silico data sets generated for four additional test conditions. Models 1 and 2 had nearly identical results on the first three test conditions (columns 1-3) but Model 2 significantly out-performed Model 1 on the fourth test condition (column 4).

The performance of Models' 1 and 2 on the training conditions (columns 1-3) and validation condition (column 4) are shown in Figure 5.7A. As expected by the identical $\mathrm{AIC}_{\mathrm{c}}$ score, both models were observed to accurately fit the in silico data set. To differentiate between the two models, an in silico data set was generated by the same procedure for four new conditions, listed in Table 5.3.

\begin{tabular}{|c|c|c|c|}
\hline \multicolumn{4}{|l|}{ Table 5.3. Viral Dynamics Model Test Conditions } \\
\hline $\begin{array}{l}\text { Initial } \\
\text { Condition }\end{array}$ & {$[\text { tem }]_{0}$} & {$[\text { gen }]_{0}$} & {$[\text { struct }]_{0}$} \\
\hline 1 & 10000 & 0 & 0 \\
\hline 2 & 0 & 5000 & 0 \\
\hline 3 & 500 & 5000 & 1000 \\
\hline 4 & 0 & 0 & 1000 \\
\hline
\end{tabular}

Models 1 and 2 were simulated using each of the four new test cases, as shown in Figure 5.7B, and scored against the in silico data sets. Both models yielded nearly identical results on the first three test cases. However, models 1 and 2 showed significantly different results when simulated against the fourth test case. Model 1 scored poorly, based on its $\mathrm{AIC}_{\mathrm{c}}$ value, as the struct molecules existing at $\mathrm{t}=0$ led to the up- 
regulation of gen and by extension tem. Model 2 had a better $\mathrm{AIC}_{\mathrm{c}}$ score and accurately predicted that the initial struct molecules would not up-regulate gen or tem and would be degraded out of the system. Model 2 was therefore correctly selected as the optimized mechanistic model for the general viral dynamics system. Table 5.4 compares the true model to the evolved model and its parameters.

\begin{tabular}{|c|c|}
\hline \multicolumn{2}{|c|}{ Table 5.4. Comparison of Known Equations to Generated Equations } \\
\hline Known Equations & Generated Equations \\
\hline$\frac{d[\mathrm{tem}]}{d t}=0.025[\mathrm{gen}]-0.25[\mathrm{tem}]$ & $\frac{d[\mathrm{tem}]}{d t}=0.025[\mathrm{gen}]-0.25[\mathrm{tem}]$ \\
\hline$\frac{d[\mathrm{gen}]}{d t}=[$ tem $]-0.025[\mathrm{gen}]-7.5 *$ & $\frac{d[\mathrm{gen}]}{d t}=0.99[\mathrm{tem}]-0.025[\mathrm{gen}]-$ \\
$10^{-8}[\mathrm{gen}][\mathrm{struct}]$ & $7.46 * 10^{-8}[\mathrm{gen}][\mathrm{struct}]$ \\
\hline$\frac{d[\mathrm{struct}]}{d t}=1000[\mathrm{tem}]-1.99[\mathrm{struct}]-$ & $\frac{d[\mathrm{struct}]}{d t}=2570.4[$ tem $]-5.14[\mathrm{struct}]-$ \\
$7.5 * 10^{-8}[\mathrm{gen}][\mathrm{struct}]$ & $7.46 * 10^{-8}[\mathrm{gen}][\mathrm{struct}]$ \\
\hline
\end{tabular}

\subsection{Discussion}

In this proof of principal study, an algorithm for determining the mechanistic reaction kinetic network and associated kinetic parameters of a nonlinear system using time series data was developed. The algorithm was validated by its ability to recapitulate a generic nonlinear viral dynamics model. To overcome issues of intractability, reaction kinetic theory was used to reduce the model search space by encoding models as interactions with reactants, parameters, and products where reactions could be classified as either describing consumption reactions or catalytic reactions. Convergence of the algorithm was also facilitated by seeding biochemical species mass balances with a production and consumption term as informed from domain specific knowledge. The 
algorithm did not require any prior knowledge of the relationship among measured species.

Eight total instances of model evolution were required to generate a mechanistic model of the viral dynamics system. The model went through two major intermediate steps before arriving at two final models that fit the validation condition equally well. Four scenarios using different initial conditions were used to differentiate between the two models and one was selected as the optimized mechanistic model. The parameters estimated with the evolved model were $160 \%$ of the parameters from the published model used to generate the in silico data set, and in most cases were almost identical. This means that the algorithm converged upon a sub-optimal parameter set for this model.

Interactions between large numbers of species are commonly found in biological systems; therefore it is important that a biochemical kinetics discovery algorithm be able to analyze systems of many species. The enhancements to the traditional genetic programming algorithm used in this study have the potential to carry out such an analysis. The maximum number of interactions suggested is the square of the number of species plus one, but it is possible to specify a reasonable number of interactions specific to the system under investigation that goes beyond this value if deemed appropriate. It is expected that running this algorithm for systems where a greater number of species are being considered will take longer to converge on a mechanistic mathematical model. Because of this, the published Mathematica code is designed to be monitored and terminated when a potential solution has not improved during a sufficient number 
generations. The algorithm therefore has the potential to be beneficial in generation models of complex biological systems.

Future research into the performance of this algorithm may identify ways to improve its computational efficiency and by extension its ability to converge to an optimal solution in a timely manner. One such approach may be to change the way by which parameters for potential solutions are trained and validated. In this study, data simulated using three different scenarios were used to infer solutions and a fourth was used to validate solutions by assigning a score using $\mathrm{AIC}_{\mathrm{c}}$. This score was then used to carry out evolutionary operations. Depending on the data set collected, it may be necessary or preferable to assign a portion of a data to the training data set and another portion to the validation data set, a common practice in the field of machine learning. Similarly, when multiple candidate solutions emerge, as was the case in this study, there is the potential to embed testing conditions into the algorithm. For example, a data set describing the responses of the variables of interest to a perturbation could be recorded and fed to the algorithm for this purpose. Also, another method of comparing the candidate solutions, such as the F-test, could be utilized. These approaches would replace the ad hoc testing done in this study.

Another such avenue for potential improvement will be regarding how interactions are chosen to be involved in a potential solution. Depending on the likelihood of an interaction being present in the system, a bias may be introduced that would make particular interactions more or less likely to be present in a potential solution. For example, a bias against dimerization interactions could be introduced when analyzing a system such as the one in this study where such interactions are unlikely to occur. Such 
an improvement may prove critical in facilitating convergence of larger systems to an optimized solution.

In conclusion, mechanistic models describing biological systems have the potential to guide the discovery of drug targets and therapeutic strategies. This study demonstrates a method of generating mechanistic mathematical models describing biological systems from experimental data sets. This technology has the potential to be useful in a variety of areas ranging from biomedical to biotechnological. For example, for personalized medicine applications, the tailoring of mathematical models to an individual's disease state based upon their unique biochemical kinetic markers could allow clinicians to make predictions about an individual's disease state and optimize their treatment. Biotechnologically, through the inference of bioprocesses, advances in the discovery of pathways associated with microbial fuel production, for example, could lead to novel insights into the optimization of these processes. Further, this algorithm has the potential to be useful in any application where the relationship between biochemical species is at question. 
CHAPTER 6

INFERENCE OF COHORT-SPECIFIC HIV-1 KINETICS 


\subsection{Introduction}

Human immunodeficiency virus type $1(\mathrm{HIV}-1)$ is an important disease that has received significant interest from the modeling community (Aviran et al. 2010, Bonhoeffer et al. 1997(a,b), Burg et al. 2009, Herz et al. 1996, Nowak and May 2000, Perelson et al. 1996, Perelson 2002, Prosperi et al. 2009, Ribeiro and Bonhoeffer 2000, von Kleist et al. 2010, Wu and Zhang 2010, Zeng and Yang 2010). In recent years, HIV1 treatments have significantly reduced AIDS-related mortalities and have enhanced long-term disease control (Dybul et al. 2001, Fagard et al. 2003, Powderly 2002, Ruiz et al. 2001). These benefits have been realized in large part via use of highly active antiretroviral therapy (HAART) in clinical practice. The HAART strategy consists of treating the disease with a combination of antiretroviral drugs. Despite advances in efficacy over prior generation treatments, HAART is associated with risk of serious side effects that include cardiovascular disease (Domingos et al. 2009), nephrotoxic effects (Izzedine et al. 2009), and oral toxicities (Nittayananta et al. 2010). Up to $16 \%$ of patients require modification or discontinuation of their HAART regimen due to drugrelated toxicities (Mocroft et al. 2005).

A major goal of developing mathematical models of diseases such as HIV-1 is to understand the underlying pathology and to predict progression of the disease (Bogojeska et al. 2010, Burg et al. 2009, Castiglione and Paci 2010, de Sousa and Cunha 2010, Degon et al. 2008, Guedj and Neumann 2010, Hoffmann et al. 2002, Itakura et al. 2010, Perelson et al. 1996, Smith and Ribeiro 2010, Srivastava et al. 2002, von Kleist et al. 2010, Wendelsdorf et al. 2010). One of the benefits arising from such a quantitative 
understanding is the potential to develop and optimize treatment strategies that maximize health benefits while minimizing secondary toxicity effects.

Mathematical models of biochemical systems, such as HIV-1 pathogenesis, are often defined by making assumptions as to the important reactions that consume and produce the variables of interest. Reaction kinetic theory is then applied to formulate mathematical models of these systems where reaction parameters are optimized using experimental data sets. Therefore, many models of various sizes and structures have been proposed for such systems taking into account a wide range of variables. Depending on the variables taken into account and their predicted relationships, a system's model behavior can vary in response to perturbation.

A more robust approach to determine a mathematical model describing a biochemical system, such as HIV-1 pathogenesis, would be to assume no prior knowledge about the system and the way that the variables of interest interact. Therefore, it would be useful to leverage an evolutionary algorithm (Blickle and Lothar 1995, Chattopadhyay et al. 2013, Costa and Oliveira, Iba 2008, Koza 1992, Schmidt and Lispon 2009) to derive a reaction kinetic model describing the variables of interest, taking into account all necessary variable interactions including some that may not be inherently obvious. The resulting model could then be used to make predictions about the system as well as to test and optimize potential therapeutics.

In this study, an evolutionary algorithmic approach was utilized to generate reaction kinetic models from clinical data describing HIV-1 infection in three cohorts of HIV-1 positive subjects receiving three different HAART regimens. These cohort-specific models allowed for the analysis of HIV-1 pathogenesis in each cohort as active 
biochemical pathways existing between $\mathrm{CD} 4^{+}$T-cells and HIV-1 were hypothesized. The inferred pathways were used to evaluate HAART effectiveness in each cohort as well as to makes predictions about the potential for viral rebound. Significantly, this study demonstrates a method by which reaction kinetics models describing HIV-1 pathogenesis can be inferred directly from clinical data.

\subsection{Methods}

\subsubsection{Selection of Subject Data}

$\mathrm{CD}^{+}$T-cell and HIV-1 data describing eight HIV-1 positive male subjects was taken from Zhang et al. (Zhang et al. 1999). Subjects 1, 2, and 5 (Cohort 1) received 600 mg/day Zidovudine, $300 \mathrm{mg}$ /day Lamivudine, and $1200 \mathrm{mg}$ /day Ritonavir, subjects 3, 4, and 6 (Cohort 2) received $600 \mathrm{mg} /$ day Zidovudine, $300 \mathrm{mg} /$ day Lamivudine, and 2400 $\mathrm{mg}$ /day Indinavir, and subjects 7 and 8 (Cohort 3) received $600 \mathrm{mg} /$ day Zidovudine, 300 mg/day Lamivudine, 1200 mg/day Ritonavir, and 1200 mg/day Saquinavir. Since plasma viremia was not detected after the $5^{\text {th }}$ month of treatment, only data from time $=0$ to approximately the $5^{\text {th }}$ month of treatment was considered for analysis. This data is depicted in Figure 6.1. 
A) Cohort I
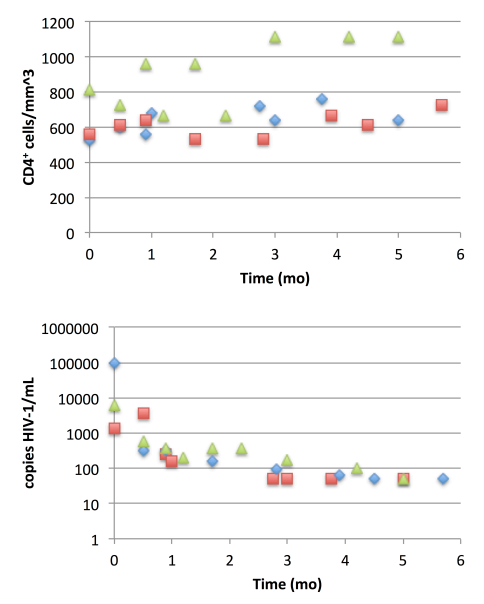

$\square$ Patient1 $\diamond$ Patient2 $\triangle$ Patient5
B) Cohort 2
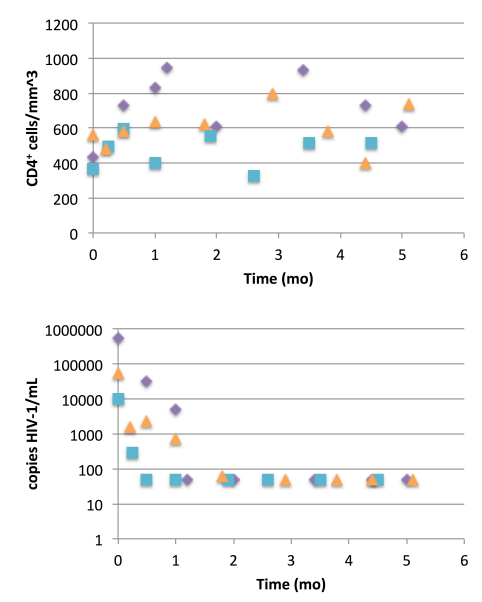

Patient3 $\square$ Patient4 $\triangle$ Patient6

\section{C) Cohort 3}
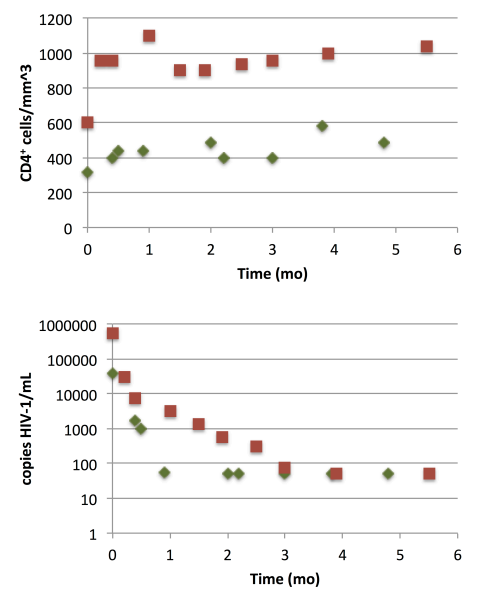

Patient7 - Patient8

Figure 6.1. $\left[\mathrm{CD}^{+}\right]$and $[\mathrm{HIV}-1]$ Data Describing HIV-1 Positive Subject Cohorts. CD $4^{+} \mathrm{T}-$ cell and HIV-1 concentration data from eight HIV-1 positive subjects was obtained from (Zhang et al. 1999). Only the first five months of data were considered as this was the only period in (Zhang et al. 1999) during which significant changes in [CD4 $\left.{ }^{+}\right]$and [HIV-1] were observed. The subjects were re-organized into three cohorts, Cohort 1 (Subjects 1, 2, and 5), Cohort 2 (Subjects 3, 4, and 6), and Cohort 3 (Subjects 7 and 8), where the subjects making up each cohort each received the same treatment regimen.

\subsubsection{Optimization of Models Using an Evolutionary Algorithm}

Reaction kinetic models of ordinary differential equations describing the change in both $\left[\mathrm{CD}^{+}\right]$and $[\mathrm{HIV}-1]$ over time were evolved using the algorithm depicted in Figure 6.2. This algorithm is described in depth in Chapter 5. Data sets for Cohorts 1, 2, and 3 were used as input to evaluations of the algorithm that resulted in a model specific for that cohort. Model optimization steps (shown in blue and magenta in Figure 6.2) were carried out using an evolutionary algorithm with evolutionary parameters defined as follows; the population size was set to 30 models and a maximum of 150 iterations was 


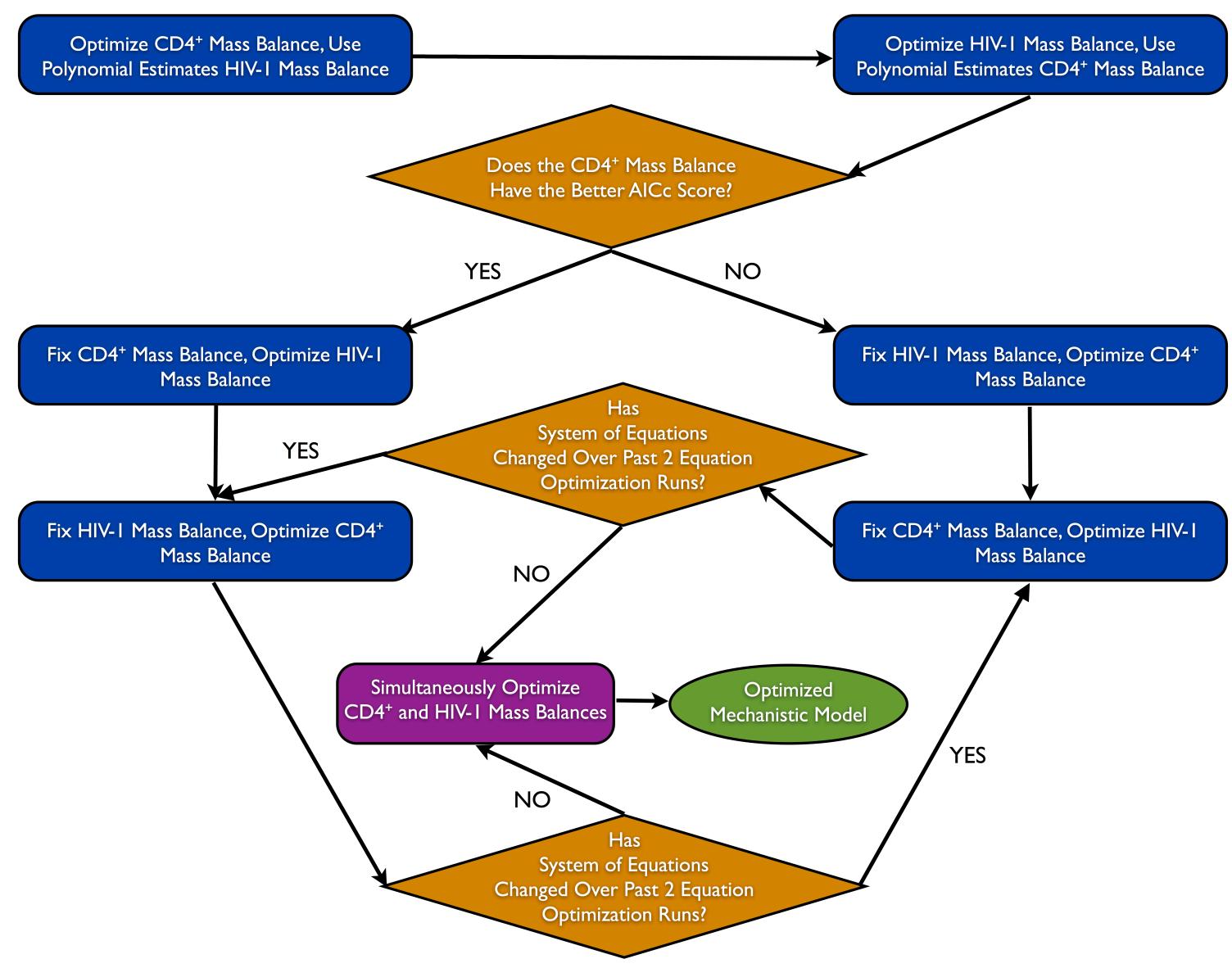

Figure 6.2. An Evolutionary Algorithmic Approach for the Optimization of Cohort-Specific

HIV-1 Infection Models. Data sets describing cohorts of HIV-1 positive subjects were used as the input to an evolutionary algorithmic approach designed to output a reaction kinetic model describing a given cohort. First, mass balances were optimized using an evolutionary algorithm for both $\mathrm{CD}^{+}$T-cells and HIV-1 individually. The best scoring mass balance, using $\mathrm{AIC}_{\mathrm{c}}$, was fixed and the mass balance for the other variable, either $\mathrm{CD}^{+}$or $\mathrm{HIV}-1$, was re-optimized. This mass balance was then fixed and the mass-balance for the other variable was again optimized. This cyclical behavior continued until both mass balances did not change over two steps. The entire model consisting of both $\mathrm{CD}^{+}$and HIV-1 mass balances was simultaneously optimized using an evolutionary algorithm with the result being the optimized reaction kinetic model. 
allowed. The recombination rate was reduced by $10 \%$ every 15 iterations, starting at $90 \%$ and ending at $0 \%$. Conversely, the mutation rate was increased by $5 \%$ every 15 iterations, starting at $5 \%$ and ending at $50 \%$.

Candidate models were represented as a table of reactions, shown in Figure 6.3. A reaction was defined by a set of reactants, a reaction parameter, and a set of products. The set of reactants and products in each reaction was randomly chosen from the set $\left\{\mathrm{CD}^{+}\right.$, HIV-1, CD4 $\left.4^{+}+\mathrm{CD}^{+}, \mathrm{HIV}-1+\mathrm{HIV}-1, \mathrm{CD}^{+}+\mathrm{HIV}-1, \mathrm{Null}\right\}$ and was randomly designated as a catalytic reaction (parameter type $=0$ ) where the reactants are not consumed in the production of the product or as a reactant consuming reaction (parameter type $=1$ ). Single equation optimization steps were capped at four terms per equation and up to five terms were allowed to make up the final model determined in the final simultaneous optimization step. 


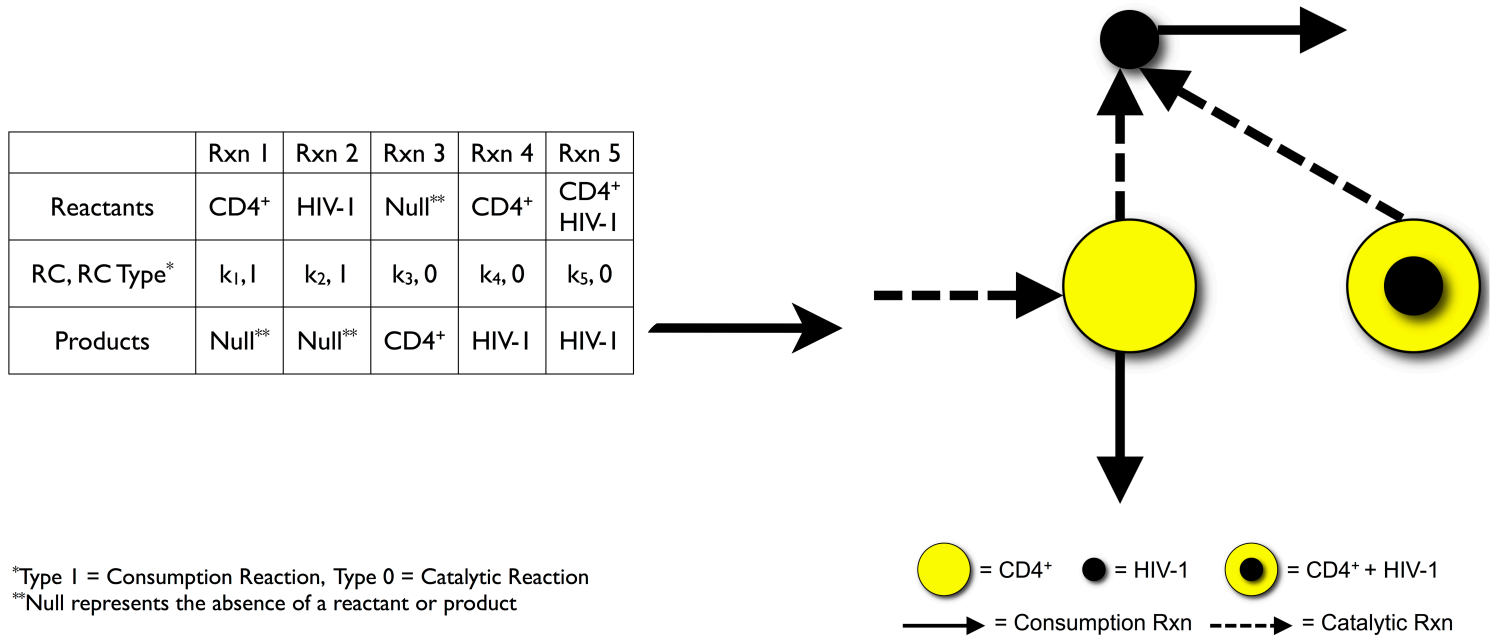

Figure 6.3. Representation of Potential Models in Evolutionary Algorithmic Approach.

During the generation of cohort-specific reaction kinetic models, evolutionary algorithms were used to build up the final model in a step-by-step fashion. These evolutionary algorithms used a population of potential models from which better scoring models emerged over several iterations. Potential models were generated as tables of reactions, where each reaction was made up of reactants, a reaction parameter (either describing a consumption or catalytic reaction), and products. Using mass action kinetic theory, a table of reactions representing a potential model was translated into a set of ordinary differential equations that could be simulated and scored against a data set (not shown). The table of reactions from an optimized model was then translated into a network diagram from which hypothesized reactions involving $\mathrm{CD} 4^{+} \mathrm{T}$-cells and HIV-1 could be visualized.

The reaction parameters describing each candidate model were optimized and each model was assigned a score using the Akaike Information Criterion with correction method (Aikaike 1974), where models that best fit the data received the lowest scores. Using an elite selection strategy, the top $20 \%$ of a scored population of models was copied unaltered to the next iteration's population and the remaining $80 \%$ of that 
population was comprised of randomly selected solutions that were given a chance to recombine and mutate to generate new and potentially better models. Eight instances of each optimization step were run and the best-scoring model from among the final populations of all eight instances was taken as the optimized solution.

\subsubsection{Evolution of Cohort-Specific Models}

During the first model optimization step of the algorithm, a mass balance was optimized for both $\left[\mathrm{CD}^{+}\right]$and $[\mathrm{HIV}-1]$ in separate runs where the mass balance for the variable not under consideration was approximated by a polynomial fit to each series of three data points. This approximation was necessary to numerically integrate the mass balance of interest and to score it against the clinical data set. At the conclusion of this step, the mass balance with the best score was fixed and the mass balance describing the other variable was optimized again. During this step and in subsequent steps, the fixed

mass balance was used during the numerical integration of the model for scoring purposes in lieu of the polynomial approximations.

The cycle of optimizing a single mass balance describing either [CD4 $\left.{ }^{+}\right]$or [HIV1] while the previously optimized mass balance describing the other variable was fixed continued until there was no change in either the $\left[\mathrm{CD}^{+}\right]$or $[\mathrm{HIV}-1]$ mass balance during the previous two steps. The model represented by these two mass balances was then used to seed the initial population of a final model optimization step where both mass balances were evolved simultaneously. The result of this step was an optimized reaction kinetic model describing reactions between $\mathrm{CD}^{+}$and HIV-1 specific to the cohort under investigation. 


\subsection{Results}

Using the evolutionary algorithmic approach, reaction kinetic models describing the change in $\mathrm{CD}^{+} \mathrm{T}$-cell and HIV-1 concentration over time were evolved for three cohorts of HIV-1 positive subjects. Data describing these cohorts was taken from Zhang et al. (Zhang et al. 1999). Cohort 1 (three subjects) received $600 \mathrm{mg} /$ day Zidovudine, 300 mg/day Lamivudine, and 1200 mg/day Ritonavir, Cohort 2 (three subjects) received 600 mg/day Zidovudine, 300 mg/day Lamivudine, and $2400 \mathrm{mg}$ /day Indinavir, and Cohort 3 (two subjects) received $600 \mathrm{mg}$ /day Zidovudine, $300 \mathrm{mg} /$ day Lamivudine, $1200 \mathrm{mg} /$ day Ritonavir, and $1200 \mathrm{mg} /$ day Saquinavir. $\mathrm{CD}^{+} \mathrm{T}$-cell data included both uninfected and HIV-1 infected cells.

Figure 6.4 shows the table of reactions and corresponding network diagrams describing each cohort's evolved model. Cohort 1's kinetic model hypothesized that both $\mathrm{CD}^{+}$T-cells and HIV-1 catalytically up-regulate production of HIV-1. Further, in one pathway $\mathrm{CD}^{+}$T-cells and HIV-1 are consumed to yield $\mathrm{CD}^{+}{ }^{+}$T-cells and in another pathway they catalytically up-regulate $\mathrm{CD}^{+} \mathrm{T}$-cell production. These pathways suggest an active infection, as presumably HIV-1 infected $\mathrm{CD}^{+}{ }^{+}$-cells catalytically produce new HIV-1, consistent with how HIV-1 is replicated. Similarly, the catalytic production of HIV-1 by HIV-1 pathway represents infection of uninfected CD4 ${ }^{+}$T-cells, whose concentration is approximately constant and factored into the reaction parameter, and replication within these newly infected cells. Finally, the second order pathway describing catalytic up-regulation of $\mathrm{CD}^{+}$T-cells by $\mathrm{CD} 4^{+}$T-cells and HIV-1 most likely represents an infection pathway, as the concentration of HIV-1 is not being reduced. Evidence of active treatment is shown in the second order pathway describing 
production of $\mathrm{CD} 4^{+} \mathrm{T}$-cells by the consumption $\mathrm{CD} 4^{+} \mathrm{T}$-cells and HIV-1. In this reaction, HIV-1 enters the T-cell but due to the reverse transcriptase inhibitor cannot effectively replicate or due to the protease inhibitor yield non-infectious HIV-1.

\section{A) Cohort I}

\begin{tabular}{|c|l|l|l|l|}
\hline & Rxn I & Rxn 2 & Rxn 3 & Rxn 4 \\
\hline Reactants & $\begin{array}{l}\text { CD4 } \\
\text { HIV-I }\end{array}$ & CD4 & HIV-I & $\begin{array}{l}\text { CD4 } \\
\text { HIV-I }\end{array}$ \\
\hline RC, RC Type & $\mathrm{k}_{1}, 0$ & $\mathrm{k}_{2}, 0$ & $\mathrm{k}_{3}, 0$ & $\mathrm{k}_{4}, \mathrm{I}$ \\
\hline Products & CD4 & HIV-I & HIV-I & CD4 $^{+}$ \\
\hline
\end{tabular}

Type I = Consumption Reaction, Type $0=$ Catalytic Reaction

\section{B) Cohort 2}

\begin{tabular}{|c|c|c|c|}
\hline & Rxn I & Rxn 2 & Rxn 3 \\
\hline Reactants & Null $^{* *}$ & CD4 $^{+}$ & $\begin{array}{l}\text { CD4 } \\
\text { HIV-I }\end{array}$ \\
\hline RC, RC Type & $\mathrm{k}_{1}, 0$ & $\mathrm{k}_{2}, \mathrm{I}$ & $\mathrm{k}_{3}, \mathrm{I}$ \\
\hline Products & $\mathrm{CD}^{+}$ & Null $^{* *}$ & Null $^{* *}$ \\
\hline
\end{tabular}

C) Cohort 3

\begin{tabular}{|c|c|c|}
\hline & Rxn I & Rxn 2 \\
\hline Reactants & $\begin{array}{l}\mathrm{CD}^{+} \\
\mathrm{CD}^{+}\end{array}$ & $\begin{array}{l}\text { CD4 }^{+} \\
\text {HIV-I }\end{array}$ \\
\hline RC, RC Туре* & $\mathrm{k}_{\mathrm{l}}, \mathrm{l}$ & $\mathrm{k}_{2}, \mathrm{l}$ \\
\hline Products & Null** & $\mathrm{CD}^{+}$ \\
\hline
\end{tabular}

Figure 6.4. Evolved Cohort-Specific Reaction Kinetic Models. Reaction kinetic models involving CD4 ${ }^{+}$T-cells and HIV-1 were evolved for Cohorts 1 (A), 2 (B), and 3 (C) using an evolutionary algorithmic approach. In the evolutionary algorithmic approach, models were represented as tables of reactions, where each reaction was made up of reactants, a reaction parameter (either describing a consumption or catalytic reaction), and products. The table of reactions was then used to generate a network diagram describing the mechanism hypothesized by each model.

Cohort 2's kinetic model hypothesized that $\mathrm{CD}^{+}$T-cells are constitutively produced in one pathway and are eliminated in another pathway. In a third pathway, $\mathrm{CD}^{+}$T-cells and HIV-1 are eliminated together. This system of reactions suggested that treatment was effective. For example, constitutive production of $\mathrm{CD}^{+}{ }^{+} \mathrm{T}$-cells as well as their elimination are pathways expected in a healthy subject. Further, the second order interaction of $\mathrm{CD}^{+}$T-cells and HIV-1 elimination suggests that infected $\mathrm{CD} 4^{+}$T-cells are detected and eliminated by the immune system. 
Cohort 3's kinetic model hypothesized that $\mathrm{CD}^{+}$T-cells are eliminated in a second order reaction. Also, $\mathrm{CD}^{+}{ }^{+}$T-cells and HIV-1 are consumed to yield $\mathrm{CD} 4^{+} \mathrm{T}$ cells. These pathways also suggest effective treatment as elimination of uninfected $\mathrm{CD} 4^{+}$ T-cells is expected in healthy individuals and elimination of HIV-1 infected $\mathrm{CD} 4{ }^{+} \mathrm{T}$-cells is beneficial in clearing the infection. Further, as seen in Cohort 1, production of $\mathrm{CD} 4^{+} \mathrm{T}$ cells by the consumption $\mathrm{CD}^{+}{ }^{+}$T-cells and HIV-1 suggests that HIV-1 entering the T-cell does not effectively replicate due to the action of reverse transcriptase and protease inhibitors.

Table 6.1. Cohort-Specific Model Reaction Parameters
\begin{tabular}{|c|c|c|c|c|c|c|c|c|}
\hline \multicolumn{3}{|c|}{ Cohort 1 } & \multicolumn{3}{c|}{ Cohort 2 } & \multicolumn{3}{c|}{ Cohort 3 } \\
\hline $\mathrm{k}_{1}$ & $7.47^{*} 10^{-6}$ & $1 /\left(\mathrm{H}^{*} \mathrm{t}\right)$ & $\mathrm{k}_{1}$ & 69.22 & $\mathrm{C} / \mathrm{t}$ & $\mathrm{k}_{1}$ & 0.018 & $1 /\left(\mathrm{C}^{*} \mathrm{t}\right)$ \\
\hline $\mathrm{k}_{2}$ & 7.35 & $\mathrm{H} /\left(\mathrm{C}^{*} \mathrm{t}\right)$ & $\mathrm{k}_{2}$ & $1.58^{*} 10^{-8}$ & $1 / \mathrm{t}$ & $\mathrm{k}_{2}$ & 0.045 & $1 /\left(\mathrm{C}^{*} \mathrm{t}\right)$ \\
\hline $\mathrm{k}_{3}$ & 158.06 & $1 / \mathrm{t}$ & $\mathrm{k}_{3}^{*}$ & $2.21^{*} 10^{-5} /$ & $1 /\left(\mathrm{H}^{*} \mathrm{t}\right)$ & & & \\
\hline $\mathrm{k}_{4}$ & 0.26 & $1 /\left(\mathrm{C}^{*} \mathrm{t}\right)$ & & $3.36^{*} 10^{-2}$ & & & & \\
\hline
\end{tabular}

$\mathrm{C}=$ cells $/ \mathrm{mm}^{3} ; \mathrm{H}=$ copies $\mathrm{HIV}-1 / \mathrm{mL} ; \mathrm{t}=$ months

* The reaction parameter was evolved to be $2.21 * 10^{-5}$ in the $\mathrm{CD}^{+}$mass balance and $3.36^{*} 10^{-2}$ in the HIV-1 mass balance.

The parameters evolved for each cohort's reaction kinetic model are shown in Table 6.1. Figure 6.5 shows the results of simulating these models with the evolved parameters and plotting the results with each cohort's data set. Models for Cohort 1 and Cohort $2, \mathrm{AIC}_{\mathrm{c}}=93.54$ and $\mathrm{AIC}_{\mathrm{c}}=115.56$ respectively, show good agreement with the HIV-1 data set. HIV-1 concentration fell to undetectable limits in both Cohort 1 and Cohort 2, consistent with the models, which hypothesized active treatment pathways. Cohort 1's model showed adequate agreement with the $\mathrm{CD}^{+}{ }^{+} \mathrm{T}$-cell data set but Cohort 2's model did not show a strong correlation. $\mathrm{CD}^{+}{ }^{+} \mathrm{T}$-cell concentration is predicted to 
plateau in Cohort 1 where as in Cohort 2 the $\mathrm{CD}^{+} \mathrm{T}$-cell concentration is predicted to climb over the five-month time frame. This is most likely due to the active infection pathways hypothesized for Cohort 1.

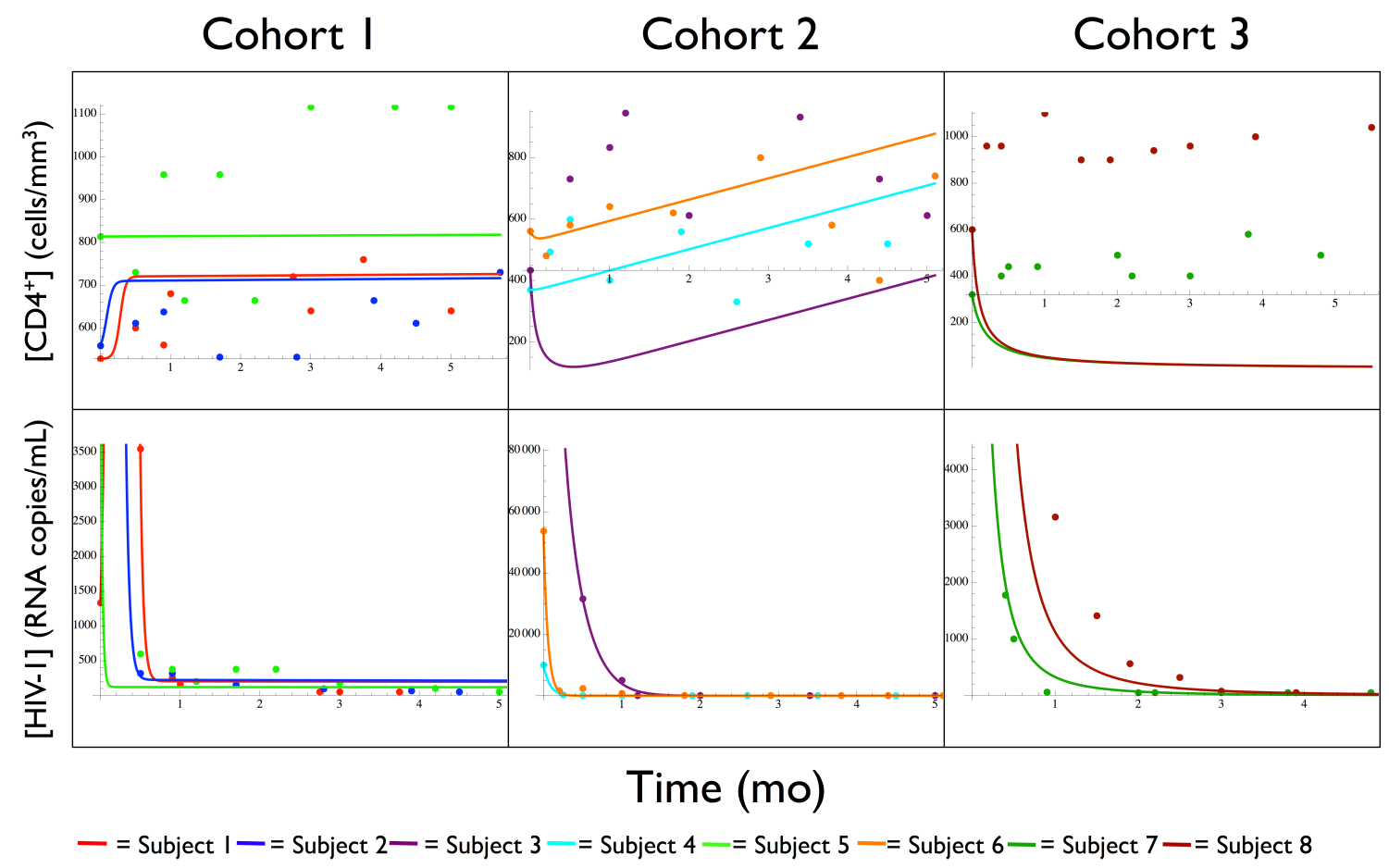

Figure 6.5. Cohort Model Simulations and Comparison To Clinical Data. Evolved models for Cohort 1 (Subjects 1, 2, and 5), Cohort 2 (Subjects 3, 4, and 6), and Cohort 3 (Subjects 7 and 8) were simulated and plotted with the clinical data set obtained from (Zhang et al. 1999). Models for Cohorts $1\left(\mathrm{AIC}_{\mathrm{c}}=93.54\right)$, Cohort $2\left(\mathrm{AIC}_{\mathrm{c}}=115.56\right)$, and Cohort $3\left(\mathrm{AIC}_{\mathrm{c}}=130.80\right)$ showed good agreement with the clinical HIV-1 data set. Cohort 1's model showed adequate agreement with the clinical CD4 ${ }^{+} \mathrm{T}$-cell data but Cohort 2's and 3's model did not show a strong correlation.

Cohort 3's model, $\mathrm{AIC}_{\mathrm{c}}=130.80$, showed good agreement with HIV-1 concentration data but did not closely predict $\mathrm{CD} 4^{+} \mathrm{T}$-cell concentration data due to the lack of a $\mathrm{CD}^{+} \mathrm{T}$-cell production pathway in the model. Even though a $\mathrm{CD} 4^{+} \mathrm{T}$-cell 
production pathway should exist for this cohort, the evolved model determined the $\mathrm{CD} 4^{+}$ T-cell and HIV-1 clearance pathways as most important and was therefore successful in describing the down-regulation of HIV-1 in this cohort.

\subsection{Discussion}

This work significantly demonstrates a method by which interactions involving $\mathrm{CD}^{+}$T-cells and HIV-1 can be inferred from HIV-1 positive subject cohort data. The hypothesized interactions can be used to assess the effectiveness of a drug cocktail on a cohort of subjects as well as to make predictions regarding viral rebound. In this study, an evolutionary algorithmic approach was successfully able to hypothesize pathways for three cohorts receiving different drug cocktails that showed the action of reverse transcriptase and protease inhibitors in down-regulating HIV-1 infection. It was also hypothesized that HIV-1 replication continued in Cohort 1. This result suggests that viral rebound and potentially mutation may emerge if treatment was to be continued. Interestingly, Zhang et al. found that viral replication did continue in Subject 5, a member of Cohort 1, in the months after the period of early infection used for this study (Zhang et al. 1999). Drug resistant HIV-1 strains were also found in this patient.

In addition to evolving reaction kinetic models for these three cohorts, models were evolved for each individual subject using their specific [CD4 $\left.{ }^{+}\right]$and [HIV-1] data (data not shown). Further, a reaction kinetic model was evolved to describe the cohort of all eight subjects used in the Zhang et al. study by pooling their [CD4 $\left.{ }^{+}\right]$and [HIV-1] data together (data not shown). It was found that models evolved for the individual subjects did not describe mechanism that one would expect to exist between [CD4 $\left.{ }^{+}\right]$and [HIV-1] 
but rather merely provided a means by which the data could be fit. These results were due to the limited amount of data available for each patient during the early infection period. Likewise, the model evolved using data pooled from all eight subjects also did not describe mechanism that one would expect to exist between [CD4 $\left.{ }^{+}\right]$and [HIV-1]. This result was due to a loss in data resolution, as the subjects under investigation received different treatment regimens. Therefore, the cohort models were selected for analysis.

It is anticipated that this evolutionary algorithmic method will be used to discover biochemical mechanism in HIV-1 positive cohorts as well as in patient's suffering from other like diseases. The key to success of this method is the availability of relevant data; large cohorts for whom large amounts of data exist will result in meaningful results. Additionally, this method has the potential to be applied to data taken from a single subject for which a large amount of dynamic data exists. The evolved model could then be used to predict the disease course for this individual and to optimize a personal treatment plan. 
CHAPTER 7

CONCLUSIONS AND FUTURE DIRECTIONS 


\subsection{Conclusions}

In conclusion, a GEP-based evolutionary algorithm was developed for the evolution of mass action kinetic models describing systems of biochemical species from time series data sets. These models are not built up assuming prior knowledge of how the variables of interest interact but significantly infer the system's reaction kinetics from the data set. Therefore, this algorithm should be utilized as a hypothesis-generating tool where pathways inferred using the evolutionary algorithm and previously not known to be important in the system can be experimentally verified.

It was also shown that by applying the GEP-based evolutionary algorithm cohortspecific interactions between $\mathrm{CD}^{+}$T-cells and HIV-1 could be elucidated. These interactions were used to determine the effectiveness of the HAART regimen prescribed for each cohort and to make predictions about potential HIV-1 rebound. In a clinical setting, the evolved models could be used to simulate the effectiveness of a HAART regimen or to optimize a HAART regimen for that cohort as was done in Chapter 5.

It is anticipated that this methodology will be applied to reaction kinetic models describing a variety of biochemical phenomena. Importantly, when applied to modeling disease states, the GEP-based evolutionary algorithm has the potential to be applied to evolve a model describing a subject's unique set of biochemical markers. The model could then be utilized to optimize a treatment strategy specific to that subject that would most effectively treat the pathways associated with that individual's disease state.

Application of the GEP-based evolutionary algorithm is not restricted to biochemical problems but could also be useful in discovering patterns underlying any 
time series data set. Evolved models would become very useful as a first step in making predictions about interactions between variables as well as in simulating the system.

\subsection{Future Directions}

This work opens up multiple avenues for future research. Currently, the GEPbased evolutionary algorithm assumes that all potential interactions have the same probability of being part of the optimized reaction kinetic model. In some systems of interest, for example in the evolution of the general viral dynamics model or in the evolution of the HIV dynamics model, it is known that some interactions, such as dimerization of biochemical species, are not likely to occur. Therefore, the GEP-based evolutionary algorithm could be altered to include biases that would result in an increased probability that a sub-population of interactions enter a candidate model and a lower probability that another sub-population of less likely interactions are chosen to be part of a candidate model.

Further, candidate models in the GEP-based evolutionary algorithmic approach were trained (the model's parameters were optimized) on a portion of the data set and then scored by validating each model with its optimized parameters on another portion of the data set. In the general viral dynamics study, see Chapter 5, where data was available from multiple experiments across multiple initial conditions, data from three of the four initial conditions was used as the model training data set and data from the fourth initial condition was used as the model validation data set. The training and validation of solutions in this algorithm is an area that deserves further scrutiny as more optimal ways of dividing an experimental data into training and validation data sets may yield better 
convergence on optimal solutions has the potential to greatly decrease the computational intensity involved in executing this algorithm.

Finally, most of the GEP-based evolutionary algorithm's evaluation time is spent on optimizing sets of parameters for candidate models. This step is crucial in reducing the solution space, as sub-optimal parameter sets are not considered. Currently, Mathematica's NMinimize function is used to carry out parameter optimization. Implementation of more efficient and computationally less intense methods to carry out parameter optimization may result in increased performance of the algorithm and the potential for the algorithm to be applied to systems with a large number of variables. 


\section{CHAPTER 8}

REFERENCES 
Akaike H (1974) A new way to look at the statistical model identification. IEEE Transactions on Automatic Control 19(6): 716-723.

Aprile G, Ramoni M, Keefe D, and Sonis S (2008) Application of distance matrices to define associations between acute toxicities in colorectal cancer patients receiving chemotherapy. Cancer 112:284-92.

Arnold DV, Beyer HG (2006) Optimum tracking with evolution strategies. Evolutionary Computation 14: 291-308.

Aviran S, Shah PS, Schaffer DV, Arkin AP (2010) Computational models of HIV-1 resistance to gene therapy elucidate therapy design principles. PLoS Comput Biol 6.

Azamathulla HM, and Ghani AA. (2011) Genetic Programming for Predicting Longitudinal Dispersion Coefficients in Streams. Water Resources Management, $25,1537-1544$

Bäck T (1996) Evolutionary Algorithms in Theory and in Practice. New York, NY: Oxford University Press.

Bains W, Basman A, and White C. (2004) HERG binding specificity and binding site structure: evidence from a fragment-based evolutionary computing SAR study. Progress in Biophysics \& Molecular Biology, 86, 205-233.

Baker JE (1985) Adaptive Selection Methods for Genetic Algorithms. Proceedings of an International Conference on Genetic Algorithms and Their Applications: 101-111. Bautista EJ, Zinski J, Szczepanek SM, Johnson EL, Tulman ER, Ching W, Geary SJ, Srivastava R (2013) Semi-automated Curation of Metabolic Models via Flux 
Balance Analysis: A Case Study with Mycoplasma gallisepticum. PLoS Comput Biol 9(9): e1003208.

Bazil JN, Qi F, and Beard DA. (2011) A Parallel Algorithm for Reverse Engineering of Biological Networks: Integrative Biology, 3(12), 1215-1223.

Belinsky MG, Guo P, Lee K, et al (2007) Multidrug resistance protein 4 protects bone marrow, thymus, spleen, and intestine from nucleotide analogue-induced damage. Cancer Res 67:262-8.

Blickle TaT, Lothar (1995) A Mathematical Analysis of Tournament Selection. Genetic Algorithms: Proceedings of the Sixth International Conference: 9-16.

Boerma M, Wang J, Burnett AF, Santin AD, Roman JJ, and Hauer-Jensen M (2007) Local administration of interleukin-11 ameliorates intestinal radiation injury in rats. Cancer Res 67:9501-6.

Bogojeska J, Bickel S, Altmann A, Lengauer T (2010) Dealing with sparse data in predicting outcomes of HIV combination therapies. Bioinformatics 26:2085-2092.

Bonhoeffer S, Coffin JM, Nowak MA (1997) Human immunodeficiency virus drug therapy and virus load. J Virol 71: 3275-3278.

Bonhoeffer S, May RM, Shaw GM, Nowak MA (1997) Virus dynamics and drug therapy. Proc Natl Acad Sci U S A 94: 6971-6976.

Bonhoeffer S, Nowak MA (1997) Pre-existence and emergence of drug resistance in HIV-1 infection. Proc Biol Sci 264:631-637.

Bonhoeffer S, Rembiszewski M, Ortiz GM, Nixon DF (2000) Risks and benefits of structured antiretroviral drug therapy interruptions in HIV-1 infection. Aids 14:2313-2322. 
Burg D, Rong L, Neumann AU, Dahari H (2009) Mathematical modeling of viral kinetics under immune control during primary HIV-1 infection. J Theor Biol 259: 751759.

Campagnaro E, Saliba R, Giralt S, et al (2008) Symptom burden after autologous stem cell transplantation for multiple myeloma. Cancer 112:1617-24.

Castiglione F, Pappalardo F, Bernaschi M, Motta S (2007) Optimization of HAART with genetic algorithms and agent-based models of HIV infection. Bioinformatics 23: $3350-3355$.

Castiglione F, Paci P (2010) Criticality of Timing for Anti-HIV Therapy Initiation. PLoS One 5(12):e15294.

Chattopadhyay I, Kuchina A, Süel GM, and Lipson H. (2013) Inverse Gillespie for inferring stochastic reaction mechanisms from intermittent samples: PNAS, 110(32), 12990-12995.

Costa L, Oliveira P (2003) An adaptive sharing Elitist Evolution Strategy for multiobjective optimization. Evolutionary Computation 11: 417-438.

Davey HM, and Davey CL. (2011) Multivariate Data Analysis Methods for the Interpretation of Microbial Flow Cytometric Data. High Resolution Microbial Single Cell Analytics, 124, 183-209.

De Jong KAaS, William M. (1991) An analysis of the interacting roles of population size and crossover in genetic algorithms. Lecture Notes in Computer Science 496: 3847.

de Sousa BC, Cunha C (2010) Development of mathematical models for the analysis of hepatitis delta virus viral dynamics. PLoS One 5. 
Deb K, Anand A, Joshi D (2002) A computationally efficient evolutionary algorithm for real parameter optimization. Evolutionary Computation 10: 371-395.

Degon M, Chipkin SR, Hollot CV, Zoeller RT, Chait Y (2008) A computational model of the human thyroid. Math Biosci 212:22-53.

Domingos H et al. (2009) Metabolic effects associated to the highly active antiretroviral therapy (HAART) in AIDS patients. Braz J Infect Dis 13:130-136.

Duan S, Bleibel WK, Huang RS, et al (2007) Mapping genes that contribute to daunorubicin-induced cytotoxicity. Cancer Res 67:5425-33.

Dybul M et al. (2001) Short-cycle structured intermittent treatment of chronic HIV infection with highly active antiretroviral therapy: effects on virologic, immunologic, and toxicity parameters. Proc Natl Acad Sci U S A 98:1516115166.

El-Sadr WM et al. (2006) CD4+count-guided interruption of antiretroviral treatment. New England Journal of Medicine 355:2283-2296.

Eremeev AV (2008) On complexity of optimal recombination for binary representations of solutions. Evolutionary Computation 16: 127-147.

Eron $\mathrm{J}$ et al. (2006) The KLEAN study of fosamprenavir-ritonavir versus lopinavirritonavir, each in combination with abacavir-lamivudine, for initial treatment of HIV infection over 48 weeks: a randomized non-inferiority trial. Lancet 368:47682.

Fagard C et al. (2003) A prospective trial of structured treatment interruptions in human immunodeficiency virus infection. Arch Intern Med 163:1220-1226.

Fang W, Sun J, Ding YR, Wu XJ, Xu WB (2010) A Review of Quantum-behaved 
Particle Swarm Optimization. Iete Technical Review 27: 336-348.

Ferreira C (2006) Gene Expression Programming. The Netherlands: Springer-Verlag.

Gajda Z, and Sekanina L. (2011) On Evolutionary Synthesis of Compact Polymorphic Combinational Circuits. Journal of Multiple-Valued Logic and Soft Computing, $17,607-631$.

Gallagher M, Frean M (2005) Population-based continuous optimization, probabilistic modeling and mean shift. Evolutionary Computation 13: 29-42.

Gandomi AH, Tabatabaei SM, Moradian MH, Radfar A, and Alavi AH (2011) A new prediction model for the load capacity of castellated steel beams. Journal of Constructional Steel Research, 67, 1096-1105.

Garcia F et al. (2001) The virological and immunological consequences of structured treatment interruptions in chronic HIV-1 infection. Aids 15:F29-40.

Gero JS, Kazakov V (2001) A genetic engineering approach to genetic algorithms. Evolutionary Computation 9: 71-92.

Goldberg DE (1989) Genetic Algorithms in Search, Optimization, and Machine Learning. Reading, MA: Addison Wesley.

Guedj J, Neumann AU (2010) Understanding hepatitis C viral dynamics with directacting antiviral agents due to the interplay between intracellular replication and cellular infection dynamics. J Theor Biol 267: 330-340.

Herz AV, Bonhoeffer S, Anderson RM, May RM, Nowak MA (1996) Viral dynamics in vivo: limitations on estimates of intracellular delay and virus decay. Proc Natl Acad Sci U S A 93: 7247-7251. 
Hoffmann A, Levchenko A, Scott ML, Baltimore D (2002) The IkappaB-NF-kappaB signaling module: temporal control and selective gene activation. Science 298:1241-1245.

Holland JH (1975) Adaptation in Natural and Artificial Systems. Ann Arbor, MI: University of Michigan Press.

Iba $\mathrm{H}$ (2008) Inference of differential equation models by genetic programming. Information Sciences 178: 4453-4468.

Ideker T, Galitski T, Hood L (2001) A new approach to decoding life: Systems Biology. Annu Rev Genom Hum G 2:343-372.

Itakura J et al. (2010) Reproducibility and usability of chronic virus infection model using agent-based simulation; comparing with a mathematical model. Biosystems 99:70-78.

Izzedine H, Harris M, Perazella MA (2009) The nephrotoxic effects of HAART. Nat Rev Nephrol 5:563-573.

Jain R, Knorr AL, Bernacki J, and Srivastava R (2006) Investigation of bacteriophage MS2 viral dynamics using model discrimination analysis and the implications for phage therapy. Biotechnol Prog 22:1650-8.

Jansen T, De Jong KA, Wegener I (2005) On the choice of the offspring population size in evolutionary algorithms. Evolutionary Computation 13: 413-440.

Kayacik HG, Zincir-Heywood AN, and Heywood MI. (2011) Can a good offense be a good defense? Vulnerability testing of anomaly detectors through an artificial arms race. Applied Soft Computing, 11, 4366-4383. 
Keefe DM and Gibson RJ (2007) Mucosal injury from targeted anti-cancer therapy. Support Care Cancer 15:483-90.

Kilby JM et al. (2000) Recurrence of the acute HIV syndrome after interruption of antiretroviral therapy in a patient with chronic HIV infection: A case report. Ann Intern Med 133:435-438.

Knorr AL and Srivastava R (2005) Evaluation of HIV-1 kinetic models using quantitative discrimination analysis. Bioinform 21:1668-77.

Knox JJ, Puodziunas AL, and Feld R (2000) Chemotherapy-induced oral mucositis. Prevention and management. Drugs Aging 17: 257-267.

Koza J (1992) Genetic Programming. Cambridge, MA: MIT Press.

Krishnan SM, Dixit NM (2011) Ribavirin-Induced Anemia in Hepatitis C Virus Patients Undergoing Combination Therapy. Plos Computational Biology 7.

Lalla RV, Sonis ST, and Peterson DE (2008) Management of oral mucositis in patients who have cancer. Dent Clin North Am 52: 61-77, viii.

Lardeux F, Saubion F, Hao JK (2006) GASAT: A genetic local search algorithm for the satisfiability problem. Evolutionary Computation 14: 223-253.

Ledergerber B et al. (1999) Clinical progression and virological failure on highly active antiretroviral therapy in HIV-1 patients: a prospective cohort study. Lancet 353:863-68.

Levenspiel O (1972) Chemical Reaction Engineering. $2^{\text {nd }}$ ed., New York: John Wiley \& Sons. 
Liang H, Miao HY, Wu HL (2010) Estimation of Constant and Time-Varying Dynamic Parameters of Hiv Infection in a Nonlinear Differential Equation Model. Annals of Applied Statistics 4:460-483.

Lipniacki T, Paszek P, Brasier AR, Luxon B, and Kimmel M (2004) Mathematical model of NF-kappaB regulatory module. J Theor Biol 228:195-215.

Madruga JV et al. (2007) Efficacy and safety of darunavir-ritonavir compared with that of lopinavir-ritonavir at 48 weeks in treatment-experienced, HIV-infected patients in TITAN: a randomized controlled phase III trial. Lancet 370:49-58.

Melgar S, Drmotova M, Rehnstrom E, Jansson L, and Michaelsson E (2006) Local production of chemokines and prostaglandin E2 in the acute, chronic and recovery phase of murine experimental colitis. Cytokine 35:275-83.

Mocroft A et al. (2005) Reasons for stopping antriretrovirals used in an initial highly active antiretroviral regimen: increased incidence of stopping due to toxicity or patient/physician choice in patients with hepatitis c coinfection. AIDS Res Hum Retrov 21:527-536.

Moraru, II and Loew LM (2005) Intracellular signaling: spatial and temporal control. Physiol 20:169-79.

Niscola P, Romani C, Cupelli L, Scaramucci L, Tendas A, et al. (2007) Mucositis in patients with hematologic malignancies: an overview. Haematologica 92: 222231.

Nittayananta W et al. (2010) Effects of long-term use of HAART on oral health status of HIV-infected subjects. J Oral Pathol Med 39:397-406.

Nomura T, Shimohara K (2001) An analysis of two-parent recombinations for real- 
valued chromosomes in an infinite population. Evolutionary Computation 9: 283308.

Nowak MA, Bonhoeffer S, Shaw GM, May RM (1997) Anti-viral drug treatment: dynamics of resistance in free virus and infected cell populations. J Theor Biol 184:203-217.

Nowak M, May R. (2000) Virus Dynamics: Mathematical Properties of Immunology and Virology. United States: Oxford University Press Inc., New York.

Oltean M (2005) Evolving evolutionary algorithms using linear genetic programming. Evolutionary Computation 13: 387-410.

Ortiz GM et al. (2001) Structured antiretroviral treatment interruptions in chronically HIV-1-infected subjects. Proc Natl Acad Sci U S A 98:13288-13293.

Panel on Antiretroviral Guidelines for Adults and Adolescents. Guidelines for the use of antiretroviral agents in HIV-1-infected adults and adolescents. Department of Health and Human Services. December 1, 2009; 1-161. Available at http://www.aidsinfo.nih.gov/ContentFiles/AdultandAdolescentGL.pdf. Accessed June 1, 2010. Appendix B, pgs 159-165.

Peterson DE (1999) Research advances in oral mucositis. Curr Opin Oncol 11: 261-266.

Peterson DE and Sonis ST (2001) Executive Summary. Mucosal Injury in Cancer Patients: New Strategies for Research and Treatment. J Natl Cancer Inst Monogr 29:3-5.

Peterson DE, Jones JB, and Petit RG, 2nd (2007) Randomized, placebo-controlled trial of Saforis for prevention and treatment of oral mucositis in breast cancer patients receiving anthracycline-based chemotherapy. Cancer 109: 322-331. 
Peterson DE, Srivastava R, White JR, Lalla RV, and Loew LM (2008) Systems biology of mucosal injury in cancer patients. Proc, J Clin Oncol 26:9634.

Perelson AS, Neumann AU, Markowitz M, Leonard JM, Ho DD (1996) HIV-1 dynamics in vivo: virion clearance rate, infected cell life-span, and viral generation time. Science 271:1582-1586.

Perelson AS, Nelson PW (1999) Mathematical Analysis of HIV-1 Dynamics In Vivo. SIAM Rev 41(1):3-44.

Perelson AS (2002) Modelling viral and immune system dynamics. Nat Rev Immunol 2: $28-36$.

Powderly W (2002) Long-term exposure to lifelong therapies. JAIDS 29:S28-S40.

Prosperi MCF, D'Autilia R, Incardona F, De Luca A, Zazzi M, et al. (2009) Stochastic modelling of genotypic drug-resistance for human immunodeficiency virus towards long-term combination therapy optimization. Bioinformatics 25: 10401047.

Ribeiro RM, Bonhoeffer S (2000) Production of resistant HIV mutants during antiretroviral therapy. Proc Natl Acad Sci U S A 97: 7681-7686.

Ribeiro RM et al. (2010) Estimation of the initial viral growth rate and basic reproductive number during acute HIV-1 infection. J Virol 84:6096-6102.

Rowe JE, Vose MD, Wright AH (2004) Structural search spaces and genetic operators. Evolutionary Computation 12: 461-493.

Ruiz L et al. (2001) HIV dynamics and T-cell immunity after three structured treatment interruptions in chronic HIV-1 infection. Aids 15:F19-27. 
Sanne I, Piliero P, Squires K, Thiry A, Schnittman S (2003) Results of a Phase 2 Clinical Trial at 48 Weeks (AI424-007): A Dose-Ranging, Safety, and Efficacy Comparative Trial of Atazanavir at Three Doses in Combination with Didanosine and Stavudine in Antiretroviral-Naïve Subjects. JAIDS 32:18-29.

Schaff J, Fink CC, Slepchenko B, Carson JH, and Loew LM (1997) A general computational framework for modeling cellular structure and function. Biophys $\mathbf{J}$ 73:1135- 46.

Schmidt M, and Lipson H. (2009) Distilling Free-Form Natural Laws from Experimental Data. Science, 324, 81-85.

Schwefel H-PP (1993) Evolution and Optimum Seeking. New York, NY, USA: John Wiley \& Sons, Inc. 456 p.

Shiri J, and Kisi O. (2011) Application of Artificial Intelligence to Estimate Daily Pan Evaporation Using Available and Estimated Climatic Data in the Khozestan Province (South Western Iran). Journal of Irrigation and Drainage EngineeringAsce, 137, 412-425.Afzal W, and Torkar R. (2011) On the application of genetic programming for software engineering predictive modeling: A systematic review. Expert Systems with Applications, 38, 11984-11997.

Slepchenko BM, Schaff JC, Macara I, and Loew LM (2003) Quantitative cell biology with the Virtual Cell. Trends Cell Biol 13:570-6.

Smith AM, Ribeiro RM (2010) Modeling the viral dynamics of influenza A virus infection. Crit Rev Immunol 30:291-298.

Sonis ST (2004) Oral mucositis in cancer therapy. J Support Oncol 2: 3-8. 
Sonis ST (2007) Pathobiology of oral mucositis: novel insights and opportunities. J Support Oncol 5(9 Suppl 4):3-11.

Srivastava R, You L, Summers J, Yin J (2002) Stochastic vs. deterministic modeling of intracellular viral kinetics. Journal of Theoretical Biology 218: 309-321.

Srivastava $\mathrm{R}^{*}$, White JR*, Lalla LV, Loew LM, Peterson DE (2011) Optimizing personalized treatment of oral mucositis secondary to cancer therapy through systems biology. Journal of Clinical Oncology 29 (suppl; abstr e19690). *Denotes co-first authors.

Sun FF, Lai PS, Yue G, et al (2001) Pattern of cytokine and adhesion molecule mRNA in hapten-induced relapsing colon inflammation in the rat. Inflamm 25:33-45.

Tang WJ, Wu QH (2009) Biologically inspired optimization: a review. Transactions of the Institute of Measurement and Control 31: 495-515.

Tsai HC, and Lin YH. (2011) Modular neural network programming with genetic optimization. Expert Systems with Applications, 38, 11032-11039.

Usman I, Khan A, and Chamlawi R. (2011) Employing intelligence in the embedding and decoding stages of a robust watermarking system. Aeu-International Journal of Electronics and Communications, 65, 582-588.

van der Greef J, Stroobant P, van der Heijden R (2004) The role of analytical sciences in medical systems biology. Curr Opin Chem Biol 8:559-565.

von Kleist M, Menz S, Huisinga W (2010) Drug-class specific impact of antivirals on the reproductive capacity of HIV. PLoS Comput Biol 6: e1000720. 
Wendelsdorf K, Bassaganya-Riera J, Hontecillas R, Eubank S (2010) Model of colonic inflammation: immune modulatory mechanisms in inflammatory bowel disease. J Theor Biol 264:1225-1239.

White JR and Srivastava R (2011) Genetic Algorithm-Based Optimization of HIV-1 Treatment Using Elite Selection Strategy Variant. 2011 BMES Annual Meeting, October 2011 (oral presentation).

Whitley D (1989) The GENITOR Algorithm and Selection Pressure: Why Rank-Based Allocation of Reproduction Trials is Best. Proceding of the 3rd International Conference on Genetic Algorithms: 116-121.

Wu L, Liu W, Hu XJ (2010) Joint inference on HIV viral dynamics and immune suppression in presence of measurement errors. Biometrics 66:327-335.

Wu J, Zhang M (2010) A Game Theoretical Approach to Optimal Control of Dual Drug Delivery for HIV Infection Treatment. IEEE Trans Syst Man Cybern B Cybern.

Xu Q, Chen QW, Li WF, and Ma JF. (2011) Pipe break prediction based on evolutionary data-driven methods with brief recorded data. Reliability Engineering \& System Safety, 96, 942-948.

Yang K et al. (2007) Dynamic simulations on the arachidonic acid metabolic network. PLoS Comput Biol 3:e55.

Zeng CB, Yang QG (2010) A Fractional Order HIV Internal Viral Dynamics Model. Cmes-Computer Modeling in Engineering \& Sciences 59: 65-77.

Zhang L. et al. (1999) Quantifying Residual HIV-1 Replication In Patients Receiving Combination Antiretroviral Therapy. N Eng J Med 340(21):1605-1613. 
Zykov V, Mytilinaios E, Adams B, and Lipson H. (2005) Self-reproducing machines.

Nature, 435, 163-164. 
CHAPTER 9

APPENDIX 


\subsection{Appendix 1: Elite Variant Selection Strategy Code}

\section{The following code was written in Common Lisp.}

;; Selection will take in a list of scored and sorted chromosomes as well as the score of the best sco oring chromosome from the previous generation and generate a new list of the best scoring and bred chro omosomes

(defun EVselection (chromosomes oldscore)

(let ((newpop '( )); the next-generation population

(newchroms 0 ); this variable will hold a set of recombined and mutated chromosomes

(newscore (car (car chromosomes)))) ; the score of this generation's best scoring chromosome

(if ( $<$ newscore oldscore)

;; If newscore < oldscore, use elite-variant selection

(progn

; ; The top 2 scoring chromosomes are copied to newpop

(do $((\mathrm{i} 1(+\mathrm{i} 1)))$

$((>$ i 2))

(push (nth (- i 1) chromosomes) newpop))

; ; The rest of newpop is made up of pairs of chromosomes where a random chromosome was chosex $\mathrm{n}$ to be recombined with one of the top 2 scoring chromosomes and both new chromosomes were given a chaw nce to be mutated

(do $((\mathrm{i} 1(+\mathrm{i} 1)))$

((> i (/ (- (length chromosomes) 2) 2)))

(progn

;; The evrecombination function gurantees recombination of chromosomes

s)

(setf newchroms (mutation (evrecombination (nth (random (length chromosomes)) chromosome»

))

(nth (random (length chromosomes)) chromosomes)

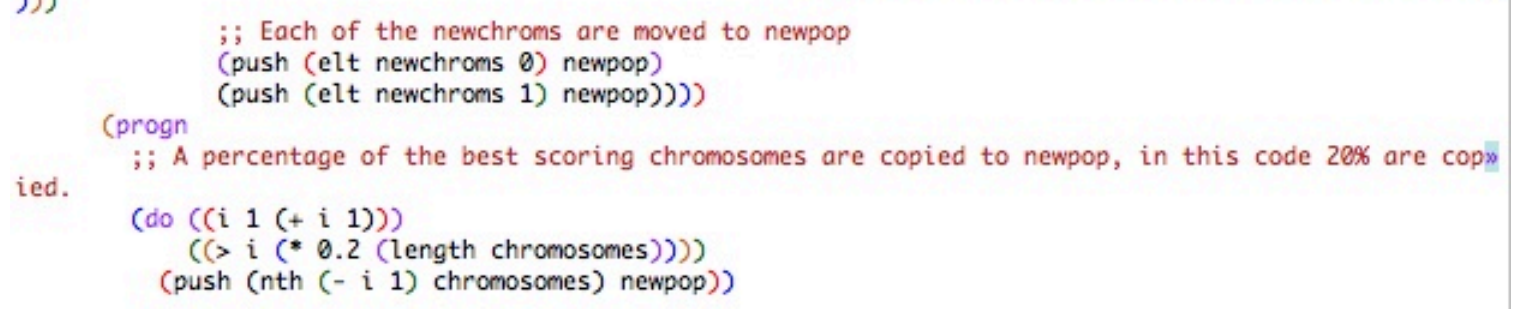

6; The rest of newpop is made up of pairs of chromosomes where a random chromosome was chosen $\%$ and given a chance to be recombined with another random chromosome and both new chromosomes were givenx a chance to be mutated

(do $((\mathrm{i} 1(+\mathrm{i} 1)))$

$((>\mathrm{i}(/(* 0.8$ (length chromosomes)) 2)))

(progn

; ; The recombination functon gives the selected chromosomes a defined chance(percentage) ox $f$ recombining. If not chosen to recombine, the chromosomes are given a chance to be mutated and then mo oved to the newpop.

(setf newchroms (mutation (recombination (nth (random (length chromosomes)) chromosomes)

(nth (random (length chromosomes)) chromosomes))) x

)

; Each of the newchroms are moved to newpop

(push (elt newchroms 0) newpop)

(push (elt newchroms 1) newpop)))))

;; The output of this function is a list containing the score of the current best scoring solutionw and the next generation population

(list newscore newpop))) 


\subsection{Appendix 2: In silico HIV-1 Positive Subject Population Analysis Code}

The following code was written in Common Lisp.

;;; ; This program seeks to calculate the range of coverage of a theoretical HAART regimen on an in sil» ico population of 1,000 HIV subjects.

;; ; The roctrial function takes as input a percentage of simulated inter-patient variation and a theor» etical treatment regimen defined as a list of daily dosages in the form of percentages of the maximum $»$ possible treatment.

(defun roctrial (varp treatment)

(let ( (coveredPats 0$)$; Number of patients covered by treatment regimen

(patients 0); This variable will hold a list of computer-generated patients

(output 0 ) ; This variable will hold simulation results for a patient

(hivdata '(O));

(do $((j 1(+j 1)))$

$((>j 10)) ; 10$ trials are carried out trial

(setf coveredPats 0 ) ; Number of patients covered by treatment regimen re-initialized for a new $\%$

(setf patients 0 ) ; in silico patient population re-initialized for a new trial

; ; The random state is re-initialized to ensure the generation of a unique population

(setf * random-state* (seed-random-state (get-time-of-day)))

(do $($ ( i $1(+\mathrm{i} 1)))$

$((>\mathrm{i} 1000)) ; 1,000$ in silico patients are evaluated (progn

; ; Treatment is simulated on a generated patient

(setf output (sim-patient (gen-patient varp) treatment i))

(if $(<$ (nth $\emptyset$ output) $\theta)$

; ; If treatment was not needed due to patients CD4+ count, nothing is tabulated nil

;; If treatment was needed, $\theta$ is added to coveredPats if [HIV] $>50$ copies/mL and 1 is addm ed to coveredPats if [HIV] $<50$ copies $/ \mathrm{mL}$

(progn

(setf coveredPats ( + coveredPats (nth 0 output)) )

(incf patients))))) ; number of total patients is tabulated

; ; List of Range of Treatment coverage and total number patients treated for this trial is backex d up to the home directory under a unique file name.

(backup (list (* (/ coveredPats patients) 100) patients) "Results" (+j (* 10 varp)))) ))

;; ; The gen-patient function takes as input a percentage of simulated inter-patient variation (defun gen-patient (varp)

(let ((basepars '(10000 0.01 0.0000005 0.5 16.75 3.1)) ; parameters taken from the Perelson Model (patientpars '())) ; variable that will hold the generated patients parameters

(dolist (par basepars (reverse patientpars))

; ; A $50 \%$ chance is given to either increase or decrease the base parameter to a number between to he base parameter and the defined limit of inter-patient variation. The output is the list of parametex rs describing the computer-generated patient.

(if (> (random 100) 50)

(push (+ par (* par (random (* .01 varp)))) patientpars)

(push (- par (* par (random (* .01 varp)))) patientpars))))) 
;;; The sim-patient function takes as an input the in silico patient's parameters, the treatment regims en, and the patients ID

(defun sim-patient (patientpars treatment pnum)

(let* (Cics '(1000000.0 0.0 100.0)) ; Patients initial levels of uninfected CD4+, infected CD4+, and» HIV

(simulation 0 ); This variable will hold the simulation results

(hivdata 'O) ; This variable will hold the patient HIV data.

(simdata 'O) ; This variable will hold one day's simulation data

$r$ not.

(treatinit 0 ); This variable will be used to determine if a patient will need to be treated ow

(time 0 ) ; This variable will tabulate time past in days.

(tco (nth 0 ics)); This variable will hold the initial uninfected [CD4+] for a one day simulaw

tion

(tso (nth 1 ics)) ; This variable will hold the initial infected [CD4+] for a one day simulati»

on

(vso (nth 2 ics))) ; This variable will hold the initial [HIV] for a one day simulation

(do $((\mathrm{i} 1(+\mathrm{i} 1)))$

((> treatinit 0$)$ )

; Infection progresses and treatment is not initiated until the patients [CD4+] falls below 500w , 000 cells $/ \mathrm{mL}$

(if (> tco 500000)

(progn

vso) 20))

(setf simulation ( $r k$ '(tc ts vs) (gen-equations patientpars $\theta$ ) '( $p 0.01 .0$ ) (list tco tso $\%$

\section{(incf time)}

;; The final variable concentrations after one day become the initial concentrations for tw he next day.

(setf tco (aref simulation 212 ))

(setf tso (aref simulation 213 ))

(setf vso (aref simulation 214 ))

(push (list time tco tso vso) simdata)

(push vso hivdata)

;; If treatment is not needed after 2 years, simulation is discontinued.

(if (> time 730)

(setf treatinit 2)) )

;; If [CD4+] falls below $500,000 \mathrm{cells} / \mathrm{mL}$, treatment is started

(incf treatinit)))

; S Simulation is discontinued if treatment is not needed for 2 years, no results reported.

(if ( $=2$ treatinit)

(progn

(list -1 (reverse hivdata)))

; Treatment is started

(progn

;; The entire treatment regimen is carried out, after which [HIV] is evaluated

(dolist (daytreat treatment)

(progn

(setf simulation ( $r k$ '(tc ts vs) (gen-equations patientpars daytreat) ' $(p 0.01 .0)$ (list tw co tso vso) 20))

(incf time)

he next day.

;; The final variable concentrations after one day become the initial concentrations for tw

(setf tco (aref simulation 212 ))

(setf tso (aref simulation 213 ))

(setf vso (aref simulation 214 ))

(push (list time tco tso vso) simdata)

(push vso hivdata)))

(if (> vso 50.0)

;; If [HIV] $>50$, patient was not covered by treatment regimen

(list $\theta$ (reverse hivdata))

;; If [HIV] $<50$, patient was covered by treatment regimen

(list 1 (reverse hivdata))) )) ))

;;; Gen-equations will generate HIV composite model equations for use in the RK simulation given a set» of parameters.

(defun gen-equations (pars dose)

' ( $\left(+\right.$, , (nth 0 pars) $\left(*-1\right.$, (nth 1 pars) tc) $\left(^{*}-1\right.$, (nth 2 pars) $(-1(* .01 .45$, dose)) vs tc))

$\left(+\left(*\left(-1{ }^{*} .01 .45\right.\right.\right.$, dose $\left.)\right)$, (nth 2 pars) vs tc) $(*-1$, (nth 3 pars) ts))

$(+(*(-1$ (* .01 .45, dose)), (nth 4 pars) ,(nth 3 pars) ts) $(*-1$, (nth 5 pars) vs)))) 


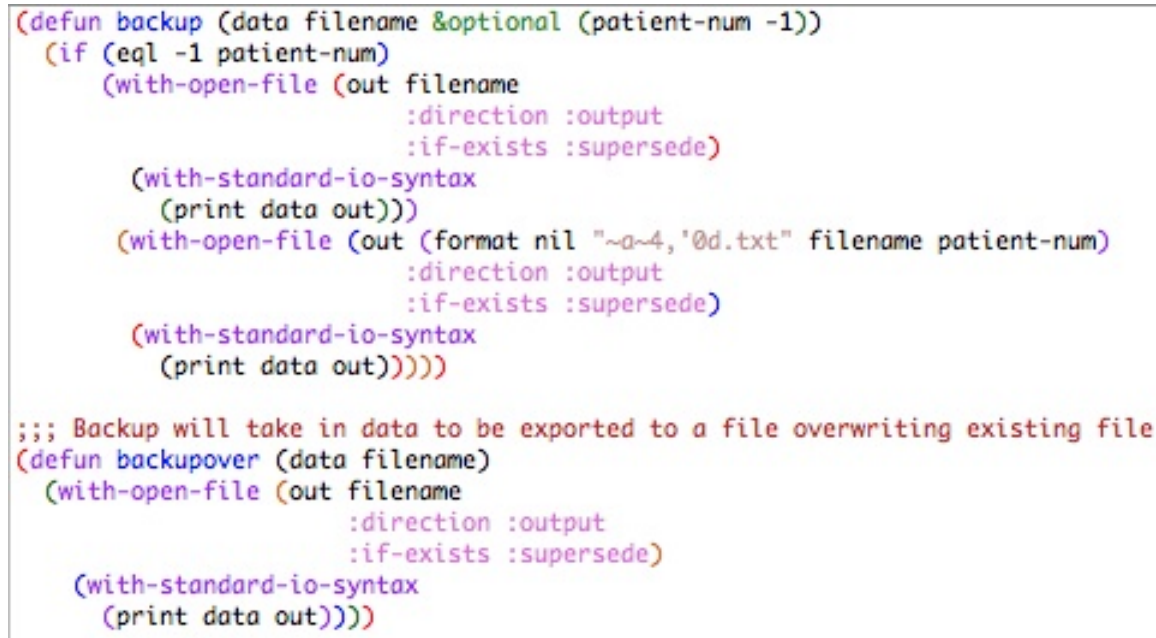

;; RK will carry out RK4 simulation given variables, equations, initial conditions, and bounds (Dr. S* rivastava's Code)

(defun rk (state-vars der ind-var init-cond \&optional (steps 10))

(declare (optimize (float 0$)$ ))

(let* ((size (length state-vars))

(all-vars (append (list (first ind-var)) state-vars))

(k (make-array 4 : initial-element 0 ))

(h (/ (- (third ind-var) (second ind-var)) steps))

(soln (make-array (list $(+2$ steps) $(+2$ size)) :initial-element 0 ))

(eqns (make-array (length der) : initial-element nil))

(k-args nil))

(dotimes (i (length der))

; Set up eqns for easy access

(setf (svref eqns i) (coerce (list 'lambda all-vars (elt der i)) 'function)))

(ar soln 00 "Step")

; Set up header and initial conditions for soln matrix

(ar soln 01 (write-to-string (first ind-var)))

(ar soln 100 )

(ar soln 11 (second ind-var))

(do $((\mathrm{i} 2$ (incf $\mathrm{i})))$

$(($ eql $i(+2$ size $)))$

(ar soln $0 \mathrm{i}$ (write-to-string (elt state-vars (- i 2))) )

(ar soln 1 i (elt init-cond $(-\mathrm{i} 2))$ ))

;Carry out calculations

; The following "do" loop increments the steps and calculation of the state-eqn vars

(do* ( $(x n$ (second ind-var) $(+x n h)$ )

(index 1 (incf index)))

(( $>$ index steps))

(ar soln (+ 1 index) 0 index)

(ar soln $(+1$ index) $1(+x n h))$

(dotimes (eqn-num (length der))

; k1 calc

(setf k-args (list (aref soln index 1))) ; set to value of $\mathrm{xn}$ (dotimes (i (length der))

(push (aref soln index $(+\mathrm{i} 2)$ ) k-args)) ; set to value of $y$ n

gs))))(

(setf (svref k0) (*h (multiple-value-call (svref eqns eqn-num) (apply \#'values (reverse k-ar») 
; $\mathrm{k} 2 \mathrm{calc}$

(setf k-args (list (+ (aref soln index 1) $(* 0.5 \mathrm{~h})$ ))) ; set to value of $x n+1 / 2^{*} h$

(dotimes (i (length der))

(push (+ (aref soln index $(+\mathrm{i} 2)$ ) (* 0.5 (svref $k 0)$ )) $k$-args)) ; set to value of $y n+1 / 2^{*} k 1$

gs)))()

(setf (svref k 1) (* $h$ (multiple-value-call (svref eqns eqn-num) (apply \#'values (reverse k-arw

; k3 calc

(setf k-args (list (+ (aref soln index 1) $(* 0.5 \mathrm{~h}))$ )) ; set to value of $x n+1 / 2 * h$

(dotimes (i (length der))

(push (+ (aref soln index $(+\mathrm{i} 2)$ ) (* 0.5 (svref $k$ 1))) k-args)) ; set to value of $y n+1 / 2 * k 2$

gs)))()

(setf (svref k 2) (*h (multiple-value-call (svref eans ean-num) (apply \#'values (reverse k-ar»

; $\mathrm{k} 4 \mathrm{calc}$

(setf $k$-args (list $(+(\operatorname{aref}$ soln index 1$) h))$ ) ; set to value of $x n+h$

(dotimes (i (length der))

(push (+ (aref soln index $(+\mathrm{i} 2)$ ) (svref k 1)) k-args)) ; set to value of $y n+k 3$

gs))())

(setf (svref k 3) (* h (multiple-value-call (svref eqns eqn-num) (apply \#'values (reverse k-ar»

;yn+1 calc

(ar soln $(+1$ index) $(+2$ eqn-num)

$(+(\operatorname{aref}$ soln index $(+2$ eqn-num)) (* $1 / 6$ (svref k 0$))(* 1 / 3($ svref k 1)) (* $1 / 3$ (svref k*

2)) $(* 1 / 6($ svref $k$ 3))) )) ) soln))

(defun ar (arr i j z)

(setf (aref arr i j) z))

;;; Function to arrange output of rk simulation in tabular form (srivastava)

(defun presult (obj)

(let* ((rows (elt (array-dimensions obj) 0))

(cols (elt (array-dimensions obj) 1))

(format $t$ " $\sim 6^{\prime \prime}$ )

(dotimes (col cols (format $t " \sim \% ")$ )

(format $t " \sim a "$ (aref obj $\emptyset$ col)) )

(dotimes (col cols (format $\left.t " \sim \% 6^{\prime \prime}\right)$ )

(format $t " \sim a "($ aref obj (- rows 1) col)))) )

;;; Function to round to decimal to $n$ digits (from online, http://coding.derkeiler.com/Archive/Lisp/cow mp.lang.lisp/2006-12/msge0845.html)

(defun froundn (float $n$ )

(multiple-value-bind (rounded frac)

(ftruncate (* float (expt $10 \mathrm{n})$ ))

$(/(+$ rounded (if $(>=$ frac 0.5$) 1.00 .0)$ )

$(\operatorname{expt} 10 n)))$ ) 


\subsection{Appendix 3: Biochemical Kinetics Inference Algorithm Code}

\subsubsection{Instructions}

The code contained in this package is intended to be used for the generation of mass action kinetic models from time series biological data sets. The four programs included in the MathematicaCode folder are as follows:

1) EvolutaionaryAlgorithm_SingleVariableStep

2) EvolutaionaryAlgorithm_SingleVariableStepComparisonOfMultipleInstances

3) EvolutaionaryAlgorithm_SingleVariableStepComparisonOfMultipleInstancesWorkAround

4) EvolutaionaryAlgorithm_CompleteModelOptimization(FinalStep)

The programs are intended to be used iteratively. First, the

EvolutaionaryAlgorithm_SingleVariableStep program is to be used to evolve mass balances describing each variable of interest separately. This means that for a 3-variable system you would use 3 different versions of this code (changing it for each variable by following the directions included with the program). It is recommended that multiple instances if each program be run to validate results.

When multiple instances are run, the EvolutaionaryAlgorithm_SingleVariableStepComparisonOfMultipleInstances program is used to identify the result that should be used to describe a variable's mass balance going forward. THE VARIABLE FOR WHICH A MASS BALANCE IS BEING EVOLVED MUST BE DEFINED AT THE HEAD OF EACH VARIABLE LIST AND BE CONSIDERED TO BE VARIABLE 1 WHEN LISTING DATA. A numerical parameter optimization function is included in this program; if the program fails to terminate in a reasonable amount of time the parameter optimization package may be failing to 
converge. In this case, the

EvolutaionaryAlgorithm_SingleVariableStepComparisonOfMultipleInstancesWorkArou nd program can be used to leverage the output from the

EvolutaionaryAlgorithm_SingleVariableStepComparisonOfMultipleInstances program to find the best result.

Once all mass balances have been found, fix the mass balance for the variable that resulted in the best (lowest) score and re-run the EvolutaionaryAlgorithm_SingleVariableStep program for the remaining variables while also defining the mass balance for the fixed variable within the code. Again, for multiple instances use

EvolutaionaryAlgorithm_SingleVariableStepComparisonOfMultipleInstances/Evolutaion aryAlgorithm_SingleVariableStepComparisonOfMultipleInstancesWorkAround programs to identify the best solution.

After mass balances have been fixed for all but one variable, run EvolutaionaryAlgorithm_SingleVariableStep/EvolutaionaryAlgorithm_SingleVariableSte pComparisonOfMultipleInstances programs to find a solution. Begin the iteratively reevolve variable mass balances in this manner beginning with the variable for which a mass balance was defined first. Iterate until the mass balances for each variable have not changed over one complete cycle.

Mass balances should now be proposed for each variable under investigation. Use the EvolutaionaryAlgorithm_CompleteModelOptimization(FinalStep) to carry out one final simultaneous evolution step. The result of this program will be a mass action kinetic 
model. Again, it is suggested that multiple instances of this program be run. The result with the best (lowest) score will be the optimized mass action kinetic model.

The following code was written in Mathematica. 


\subsubsection{EvolutionaryAlgorithm_SingleVariableStep.nb}

This program is written to evolve single variable mass balances as a step in the evolution of a mass action kinetic model from an experimental data set.

To run this program, please specify the necessary input and then evaluate the entire notebook by navigating to "Evaluation" and selecting "Evaluate Notebook".

\section{Input}

Variable for which a mass balance is being evolved

This needs to correspond to the first variable in the variables list below under System Information. Also, this variable should be considered to be Variable 1 when inputting time series data sets.

$\operatorname{var}=$ tem;

Output directory, please change to desired output location

odir = "/Users/homedir/";

If running multiple instances of the algorithm, assign a unique run identification to differentiate output

stepname = "VariableStep1", run $=" a "$;

Evolutionary Parameters

popsize $=30 ;(*$ population size $*)$

generations $=150 ;(*$ number of generations over which

the algorithm will execute, MUST BE A MULTIPLE OF 10 *)

recombinationPercentages $=\{90,80,70,60,50,40,30,20,10,0\}$;

(* percentage of solutions recombining,

as changed over the evolution of the population *)

mutationPercentages $=\{5,10,15,20,25,30,35,40,45,50\}$;

(* percentage of solutions mutating,

as changed over the evolution of the population *) 


\section{System Information}

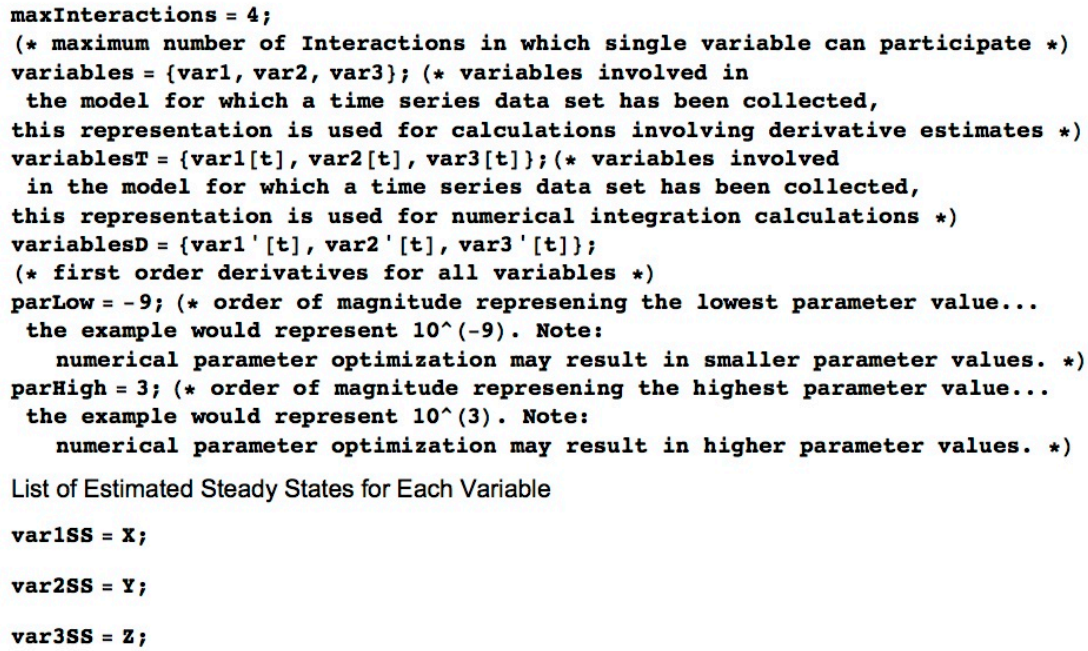

\section{Mass balances for other species}

If a mass balance has not yet been evolved for a given variable, recored the knownVariableEqn (used for calculations involving derivarive estimates) and knownVariableEqnT (used for calculations involving numerical integration) as "-1".

Note: Variable 1 below is not the same as the variable for which a mass balance is being evolved since the assumption of this algorithm is that the equation for said variable is considered unknown.

knownVariable1Eqn $=-1 ;$
knownVariable1EqnT $=-1 ;$
knownVariable2Eqn $=-1 ;$
knownVariable2EqnT $=-1 ;$

(* Continue for any other known variables *)

\section{Data $(T=$ Training Data Set, $V=$ Validation Data Set $)$}

Enter as $\{\{\{\{$ time1, conc1\}, \{time2, varconc2\}... $\}\}\}$ where the brackets represent \{entire data set $\},\{\{$ data set for an initial condition\}...\}, \{\{\{trial for an initial conition\}......\},\{\{\{timepoints, concdata\}... ... ...\}

Note: Variable numbers should correspond to variable order in the list of variables under System Informa tion above. 


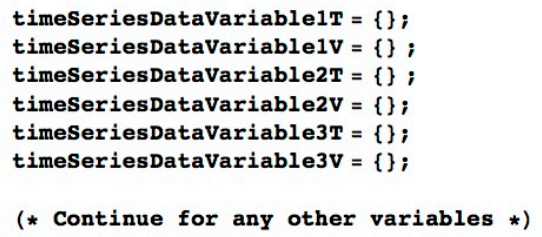

Make Data and Equations Available Across All Nodes (for parallelization tasks)

List all data sets, known equations, and steady states here so that they are availble to all nodes

DistributeDefinitions [timeSeriesDataVariable1T, timeSeriesDatavariable1v, timeSeriesDataVariable2T, timeSeriesDataVariable2v, timeSeriesDatavariable3T, timeSeriesDataVariable3v, knownVariable1Eqn, knownVariable1EqnT, knownVariable2Eqn, knownVariable2EqnT, var1ss, var2ss, var3SS];

\section{Data Preparation}

The "cpolyfit", "avdata", and "coeffit" functions are used to fit polynomials to each three consecutive data points. These polynomials can be used to estimate variable values at intermediate time points as well as to estimate derivatives at points of interest.

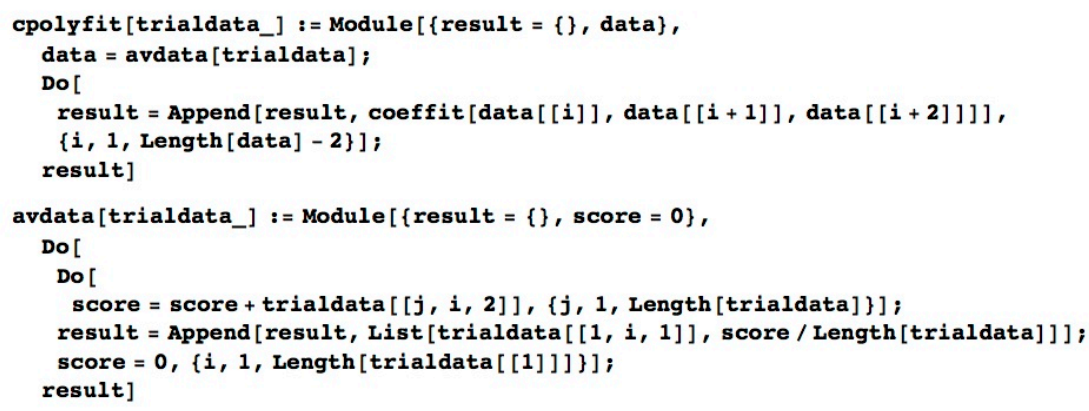




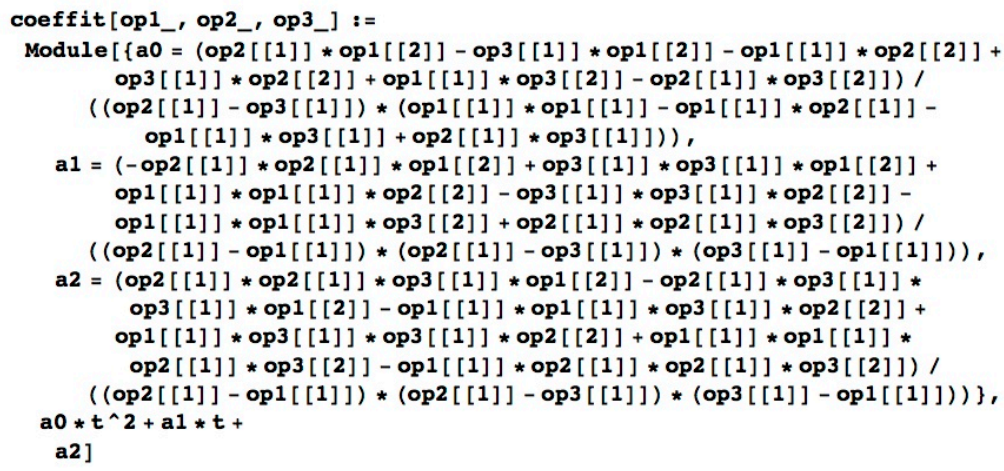

Calculation of Polynomial Sets for Each Variable

polynomialst $=\{\} ;$

Do [

polytemp $=\{\} ;$

Do [

polytemp = Append [polytemp,

cpolyfit [ToExpression ["timeSeriesDataVariable" 〈> ToString[i] 〈> "T"] [j]]]],

$\{j, 1$, Length [ToExpression [ timeSeriesDataVariable" $<>T o S t r i n g[i]<>~ " T "]]\}]$;

polynomialsT $=$ Append [polynomialsT, polytemp],$\{i, 1$, Length [variables $]\}]$

polynomialsv $=\{\}$;

Do [

polytemp $=\{\} ;$

Do [

polytemp = Append [polytemp,

cpolyfit [ToExpression ["timeSeriesDataVariable" $<$ ToString[i] 〈> "v"] [j]]]],

$\{j, 1$, Length [ToExpression [ timeSeriesDataVariable" $<>$ ToString [i] <> "v"]] ] ;

polynomialsv = Append [polynomialsv, polytemp],$\{i, 1$, Length $[$ variables $]\}]$

Calculation of Derivative Estimates for Each Variable at Each Experimental Time Point (needed only for the training data set)

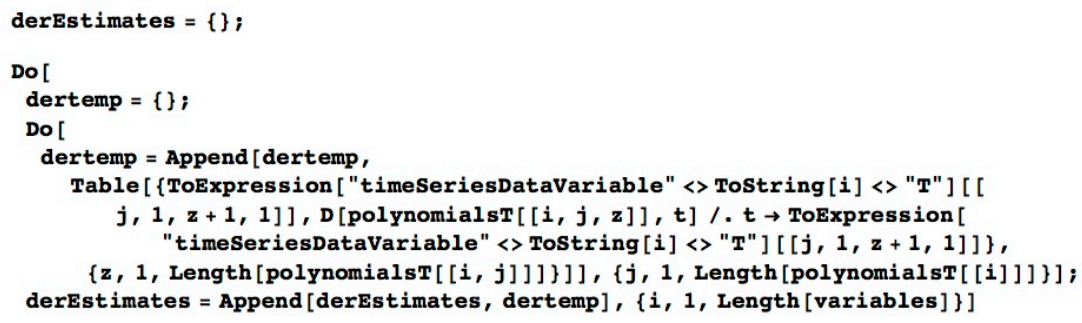




\section{Calculation of Sample Size}

This assumes that each trial of each condition includes the same number of experimental time points.

sampleSize $=$ Length $[$ timeSeriesDataVariable1T $[[1,1]]]$;

\section{Evolutionary Operations}

The "ea" function is the top level function for the execution of the evolutionary algorithm. A population of potential solutions will be generated and will undergo cycles of parameter optimization, selection, recombination, and mutation until the desired number of generation has been fulfilled.

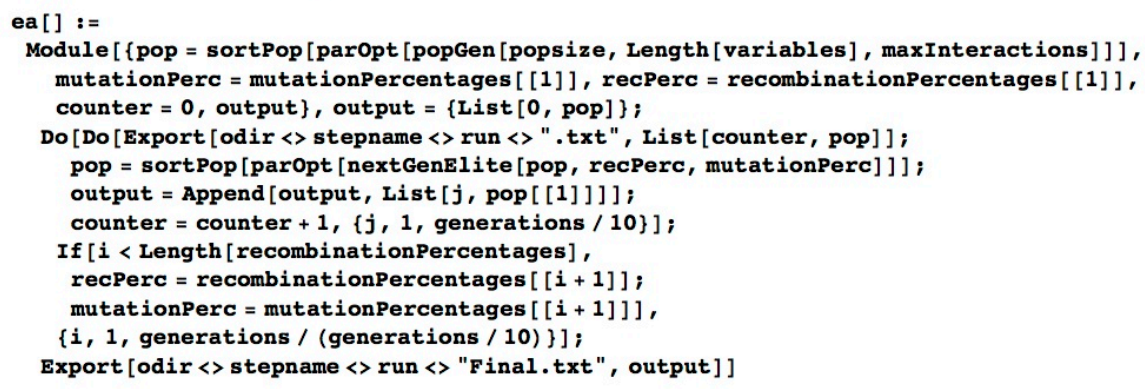

The "rc" function will generate a random parameter on a log scale within the range specified.

$\operatorname{rc}[]:=$ RandomReal $[\{$ parLow, parHigh $\}]$ 
The "popGen" function will generate an initial population of potential solutions using the specified population size, the number of variables in the system, and the maximum interactions for which the variable can be involved. The variable mass balance under analysis will be initiated with a zeroth order production term and a first order degradation term.

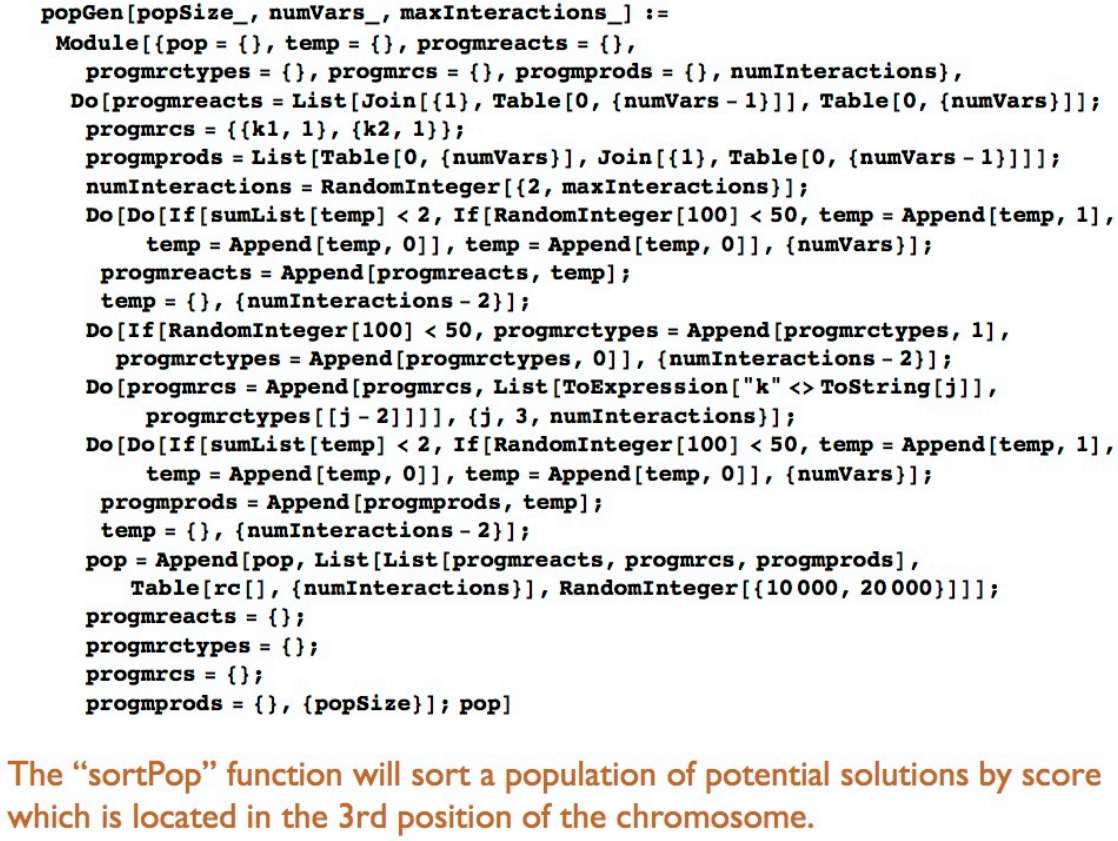

The "sortPop" function will sort a population of potential solutions by score which is located in the 3rd position of the chromosome.

sortPop [spop_] : = Sort [spop, \#1 [ [3] ] $<2[[3]] \&]$ 
The "nextGenElite" function will carry out selection, recombination, and mutation operations to populate a next generation population of solutions that contains some old solutions and some newly generated solutions. The Elite selection strategy will be used.

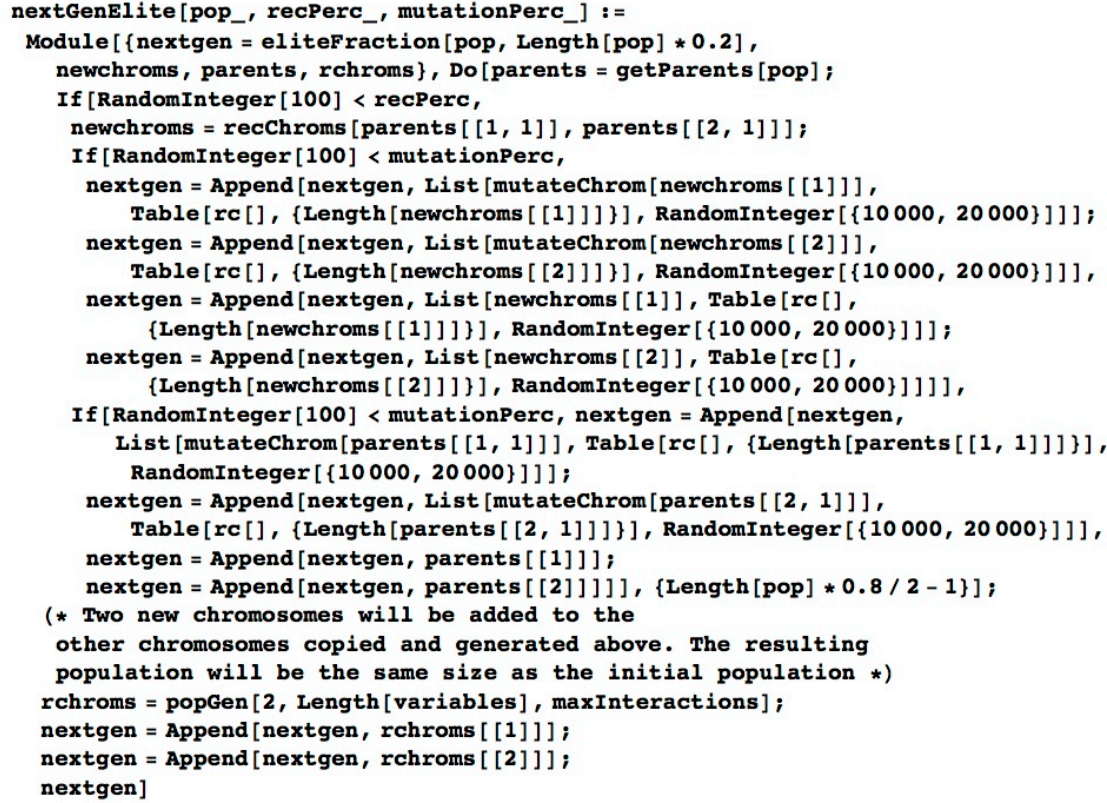

The "eliteFraction" function will take a percentage of the best scoring solution and output them to be copied unaltered to the next generation population of solutions.

eliteFraction [pop_, fractsize_] :=

Module $[\{$ result $=\{\}\}$, Do $[$ result $=$ Append $[$ result, pop $[i]]],\{i, 1$, fractsize $\}]$; result]

The "getParents" function ensures that two unique parents are selected for recombination.

getParents [pop_] : = Module $[\{$ parent $1=\operatorname{pop}[[\operatorname{RandomInteger}[\{1, \operatorname{Length}[$ pop $]\}]]]$, parent $2=\operatorname{pop}[[$ RandomInteger $[\{1$, Length $[$ pop $]\}]]]\}$, while [

parent1 [[3]] $=\operatorname{parent2}[[3]], \operatorname{parent2}=\operatorname{pop}[[\operatorname{RandomInteger}[\{1, \operatorname{Length}[$ pop $]\}]]]] ;$ \{parent1, parent2\} ] 
The "recChroms" function will recombine two chromosomes or solutions to form two new solutions.

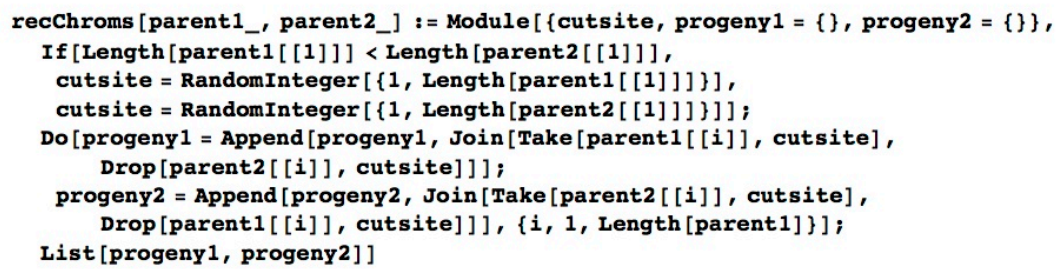

The "mutateChrom" function will make a point mutation in a solution.

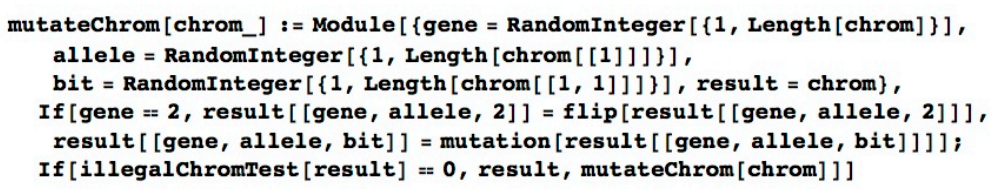

The "illegalChromTest" function will test to make sure that a point mutation does not result in an invalid chromosome that has more that 2 reactants or products.

illegalChromTest [chrom_] : = Module [ $\{$ result $=0\}$, $\operatorname{Do}[\operatorname{If}[\operatorname{sumList}[\operatorname{chrom}[[1, i]]]>2, \operatorname{result}=\operatorname{result}+1],\{i, 1, \operatorname{Length}[\operatorname{chrom}[[1]]]\}]$; $\operatorname{Do}[\operatorname{If}[\operatorname{sumList}[\operatorname{chrom}[[3, i]]]>2, \operatorname{result}=\operatorname{result}+1],\{i, 1, \operatorname{Length}[\operatorname{chrom}[[3]]]\}]$; result]

The "sumList" function will return the summation of a vector or matrix.

sumList [1st_] := Module $[\{$ sum $=0\}, \operatorname{Do}[$ If [VectorQ[1st [i] ] ] | MatrixQ[1st [i] ] ], sum $=\operatorname{sum}+\operatorname{sumList}[1 \mathrm{st}[[i]]], \operatorname{sum}=\operatorname{sum}+1 \mathrm{st}[[i]]],\{i, 1$, Length $[1 \mathrm{st}]\}]$; sum $]$

The "flip" function will mutate a "I" to a "0" or a "0" to a "I".

flip [bit_] := If $[$ bit $=1,0,1]$

The "mutation" will mutate a "0" to a "I" or "2", a "I" to a "0" or "2", or a "2" to a "0" or "I".

mutation [num_] : = Module $[\{$ new $=\operatorname{RandomInteger}[2]\}$, If $[$ num $==$ new, mutation $[$ num $]$, new $]]$

\section{Parameter Optimization}


The "parOpt" function will optimize a set of parameters for each candidate solution. For this single variable mass balance step, the best set of many randomly generated sets will be taken as the optimal parameter set.

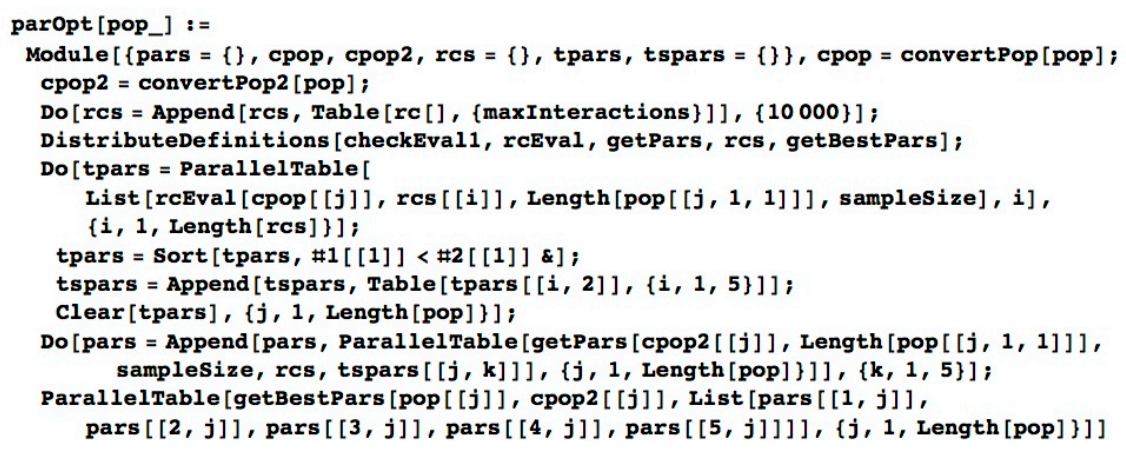

The following functions will translate a chromosome from the encoded form to a set of ordinary differential equations that can be used to directly calcuate derivative for comarison to data derivative estimates or that can be used for numerical integration and prediction of variable concentrations for direct comparison to experimental data (functions for this case end in " 2 ").

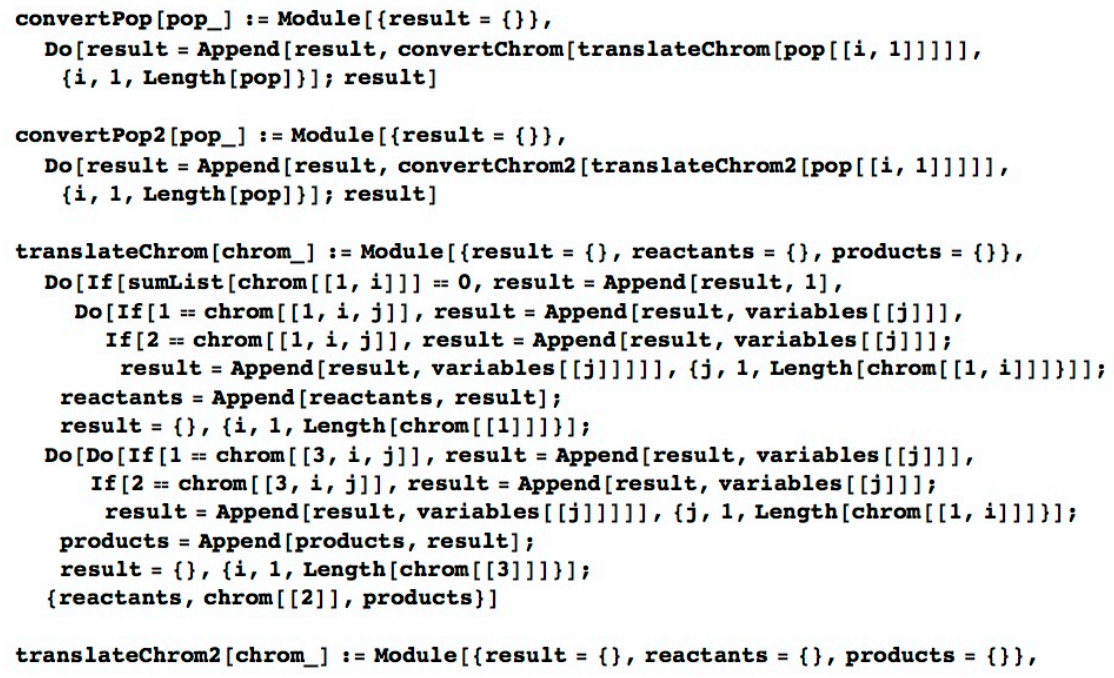




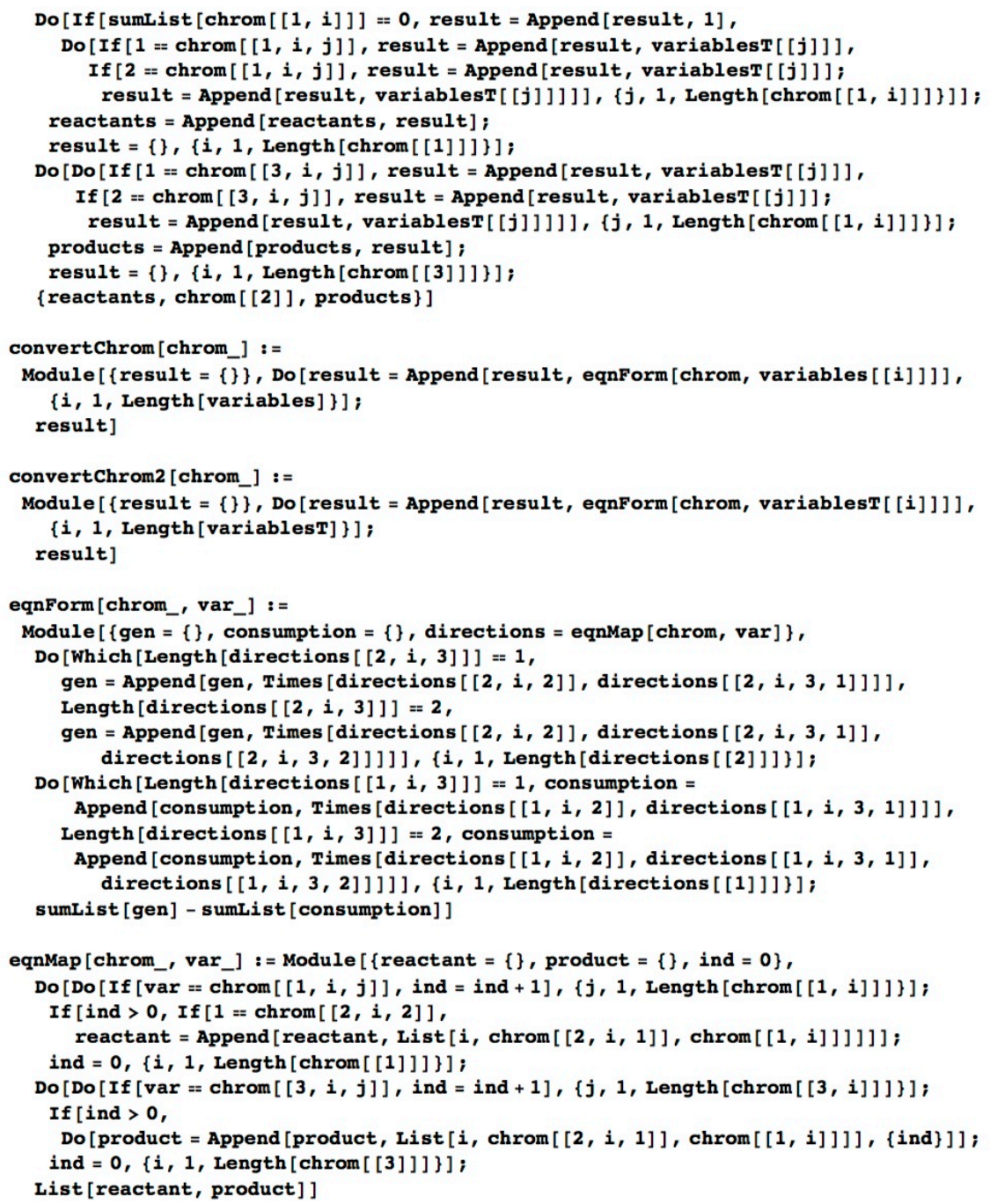

The "getPars" function will calculate the model score on the training initial conditions for the best five parameter sets from the derivative estimate calculation. 
getPars [program_, $k_{-}$, num_, rcs_, tspars_] : =

List [rcEval2 [program, rcs [ [tspars] ], k, num], rcs [ [tspars] ] ]

The "getBestPars" function will idenitfy the score of the parameter set on the validation initial condition and determine if it is better that then score of the parameter set from the previous iteration.

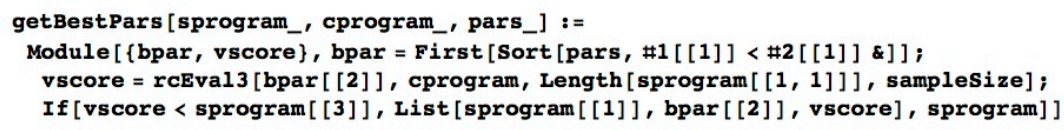

The "rcEval" function will evaluate the AICc score of a solution on the training data set by using derivative estimates.

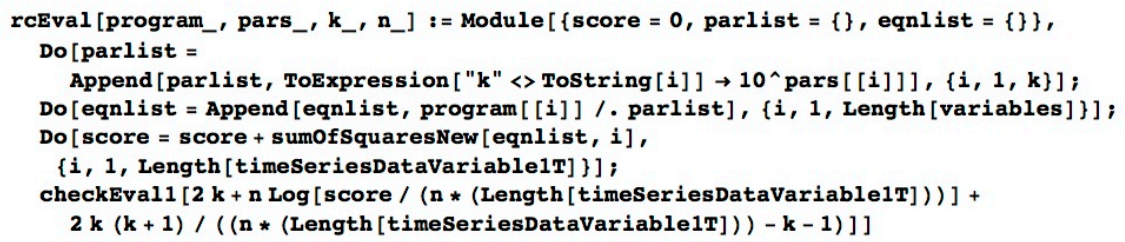


The "rcEval2" function will evaluate the AICc score of a solution on the training data set by using numerical integration of the model.

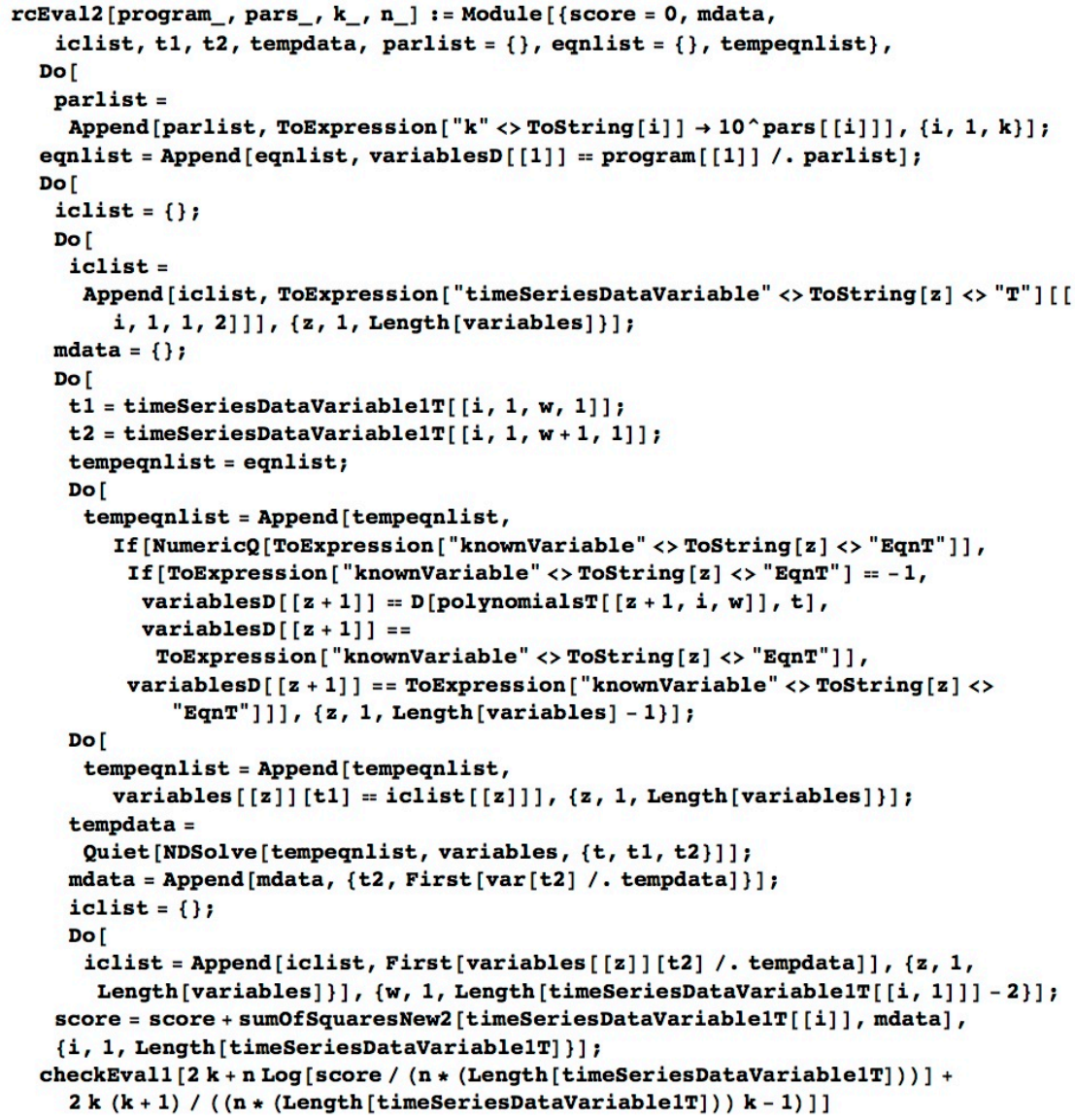


The "rcEval3" function will calculate the AICc score of a solution and its optimial parameters on the validation data set.

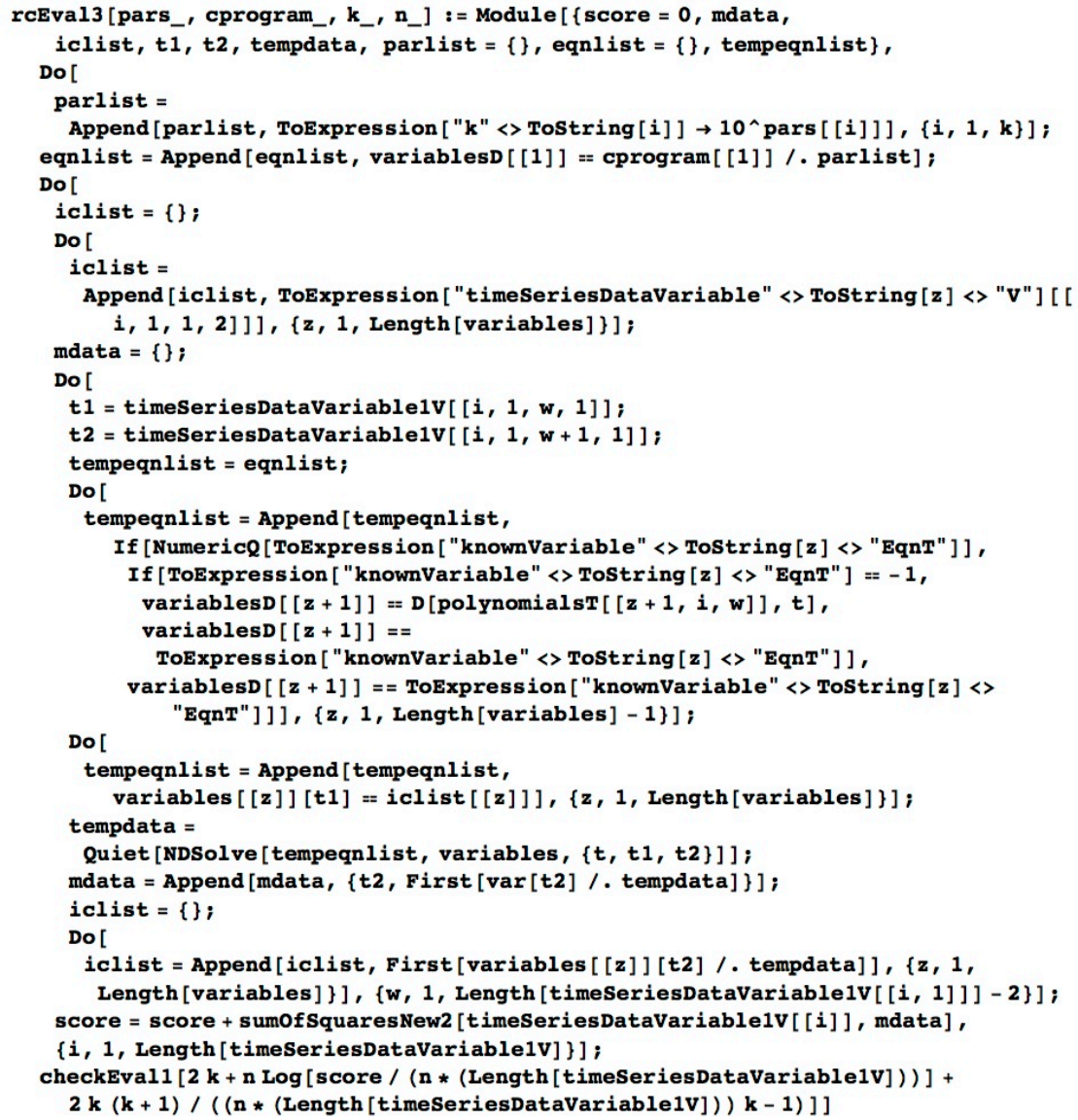

The "checkEvalI" function will replace any non-numerical score as a result of computational overflow with a very large score of $I^{*} 10^{\wedge}(100)$...this score will move a potential solution to the bottom of the population.

checkEval1 [evalResult_] : = If [NumericQ[evalResult], evalResult, $1 * 10^{\wedge} 100$ ]

The "sumOfSquaresNew" function will calculate the sum of squared differences 
between model derivate calculations and experimental data derivative estimates.

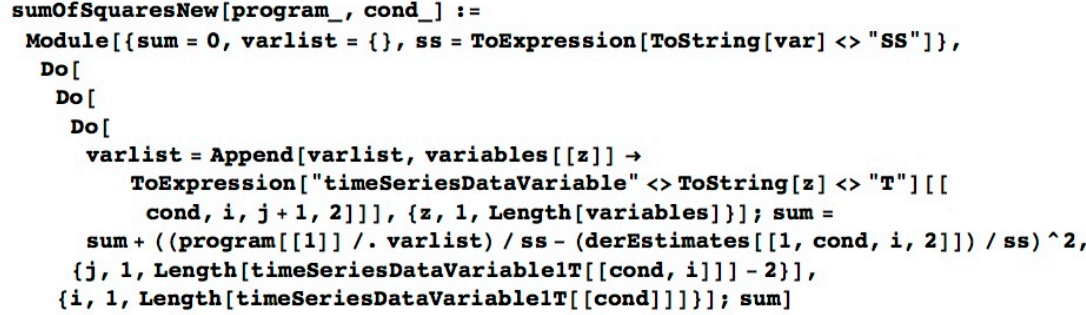

$\{i, 1$, Length [timeSeriesDataVariable1T[[cond] ] $\}]$; sum]

The "sumOfSquaresNew2" function will calculate the sum of squared differences between model data and experimental data.

sumof SquaresNew2 [expdata_, mdata_] :

Module $[\{\operatorname{sum}=0\}, \operatorname{Do}[\operatorname{Do}[\operatorname{sum}=\operatorname{sum}+(\operatorname{expdata}[[i, j+1,2]]-\operatorname{mdata}[[j, 2]]) \wedge 2$,

$\{j, 1$, Length [mdata] $\}],\{i, 1$, Length [expdata] $\}] ;$ sum]

\section{Execution of the Algorithm}

The command below will exectute the evolutionary algorithm to optimize a mass balance for the variable under consideration.

ea [ ] ; 


\title{
9.3.3 EvolutionaryAlgorithm_SingleVariableStepComparisonOfMultipleInstances.nb
}

\begin{abstract}
This program is written to identify the best solution out of a number of instances run to evolve a single mass balance as part of the algorithm to evolve a complete mass action kinetic model. This program does more than just sort the solutions by score; the parameters associated with each solution are used as initital guesses to a numerical parameter optimization funtion. The solution with the best score after this secondary parameter optimization operation is taken as the newly evolved mass balance for the variable under investigation.
\end{abstract}

If this program fails to terminate due to failure of numerical parameter optimization instances to terminate, proceed to the EvolutionaryAlgorithm_SingleVariableStepComparisonOfMultiplelnstancesWorkAround notebook and utilize output files from single program numerical optimization runs.

Also, if the output containing the optimized balance contains " $z$ " terms, please elimiate the " $z->$ " expressions and evaluate the equation to get the correct condensed equation.

To run this program, please specify the necessary input and then evaluate the entire notebook by navigating to "Evaluation" and selecting "Evaluate Notebook".

\section{Input}

Much of this information can be directly copied from the single variable instances.

Variable for which a mass balance is being evolved

This needs to correspond to the first variable in the variables list below under System Information. Also, this variable should be considered to be Variable 1 when inputting time series data sets.

$\operatorname{var}=\mathbf{x}$;

List of best solutions from each single variable mass balance instance for this step

bestSolnPop $=\{\}$;

Current solution (in interaction table form) for variable under investigation

If this is the first iteration, define solution as "-1". MAKE SURE that parameters in interaction table are listed in $\left(\mathrm{z}^{*} \rightarrow>\log\right.$ real num) form.

lastSoln $=-1$;

Output directory, please change to desired output location

odir = "/Users/homedir $/ "$;

If running multiple instances of the algorithm, assign a unique run identification to differentiate output

stepname = "VariableStep1Bestof Instances"; 


\section{System Information}

variables $=\{\operatorname{var} 1, \operatorname{var} 2, \operatorname{var} 3\} ;(*$ variables involved in

the model for which a time series data set has been collected,

this representation is used for calculations involving derivative estimates $*$ )

$\operatorname{variables} T=\{\operatorname{var} 1[t], \operatorname{var} 2[t], \operatorname{var} 3[t]\} ;(* \operatorname{variables}$ involved

in the model for which a time series data set has been collected,

this representation is used for numerical integration calculations *)

variablesD $=\left\{\operatorname{var} 1{ }^{\prime}[t], \operatorname{var2} '[t], \operatorname{var3} '[t]\right\} ;$

(* first order derivatives for all variables *)

List of Estimated Steady States for Each Variable

$\operatorname{var} 1 \mathrm{ss}=\mathrm{x} ;$

$\operatorname{var2ss}=\mathbf{Y} ;$

$\operatorname{var} 3 \mathbf{S S}=\mathbf{z} ;$

Mass balances for other species

If a mass balance has not yet been evolved for a given variable, recored the knownVariableEqn (used for calculations involving derivarive estimates) and knownVariableEqnT (used for calculations involving numerical integration) as "-1".

Note: Variable 1 below is not the same as the variable for which a mass balance is being evolved since the assumption of this algorithm is that the equation for said variable is considered unknown.

knownVariable1Eqn $=-1$;

knownVariable1EqnT $=-1$

knownVariable2Eqn $=-1$;

knownVariable2EqnT $=-1$;

(* Continue for any other known variables *)

Data $(T=$ Training Data Set, $V=$ Validation Data Set $)$

Enter as $\{\{\{\{t i m e 1$, conc1\}, \{time2, varconc2\}... $\}\}\}$ where the brackets represent \{entire data set\}, $\{\{$ data set for an initial condition\}...\}, \{\{\{trial for an initial conition $\}. ..\}, \ldots\}\{\{\{\{$ timepoints, concdata\}... ... ...\}

Note: Variable numbers should correspond to variable order in the list of variables under System Informa tion above.

timeSeriesDataVariable1T $=\{\}$;

timeSeriesDataVariable1v $=\{\}$;

timeSeriesDataVariable2T $=\{\}$

timeSeriesDataVariable2v $=\{\} ;$

timeSeriesDataVariable3T $=\{\}$

timeSeriesDataVariable3v $=\{\} ;$

(* Continue for any other variables *)

Define Functions That Are Dependant on the Maximum Number Of Interactions

The two functions below need to be definied in terms of the number of interactions under investigation. 
Please follow the directions in the ( ${ }^{*}$ comments $\left.^{*}\right)$ to definie the functions correctly. The functions are currently set up for a maximum of 10 interactions.

The "getPars" function will calculate the model score on the training initial conditions. The best five parameter sets from the derivative estimate calculation are used as the initial guesses to a parameter optimization function.

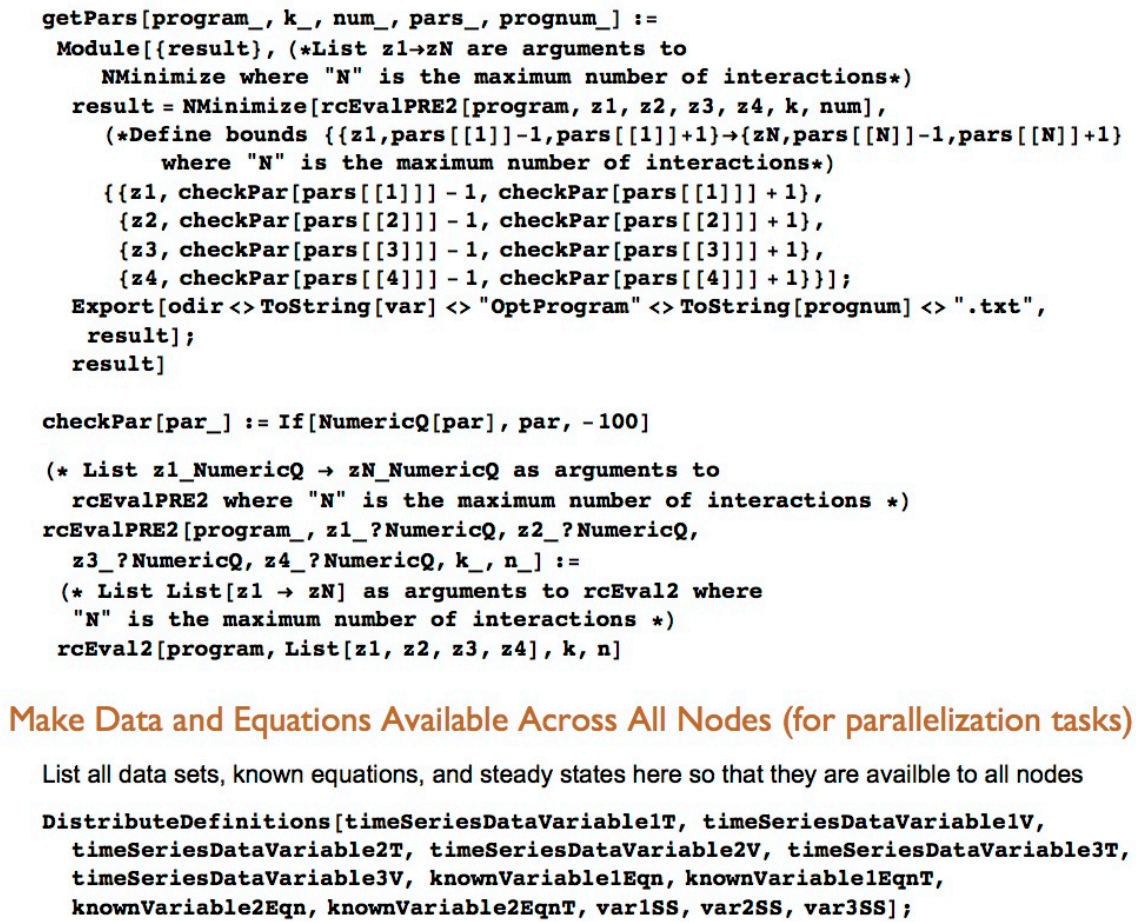

Make Data and Equations Available Across All Nodes (for parallelization tasks)

List all data sets, known equations, and steady states here so that they are availble to all nodes

DistributeDefinitions [timeSeriesDataVariable1T, timeSeriesDataVariable1v, timeSeriesDataVariable2T, timeSeriesDataVariable2V, timeSeriesDataVariable3T, timeSeriesDataVariable3v, knownVariable1Eqn, knownVariable1EqnT, knownVariable2Eqn, knownVariable2EqnT, var1sS, var2SS, var3SS] ; 


\section{Data Preparation}

The "cpolyfit", "avdata", and "coeffit" functions are used to fit polynomials to each three consecutive data points. These polynomials can be used to estimate variable values at intermediate time points as well as to estimate derivatives at points of interest.

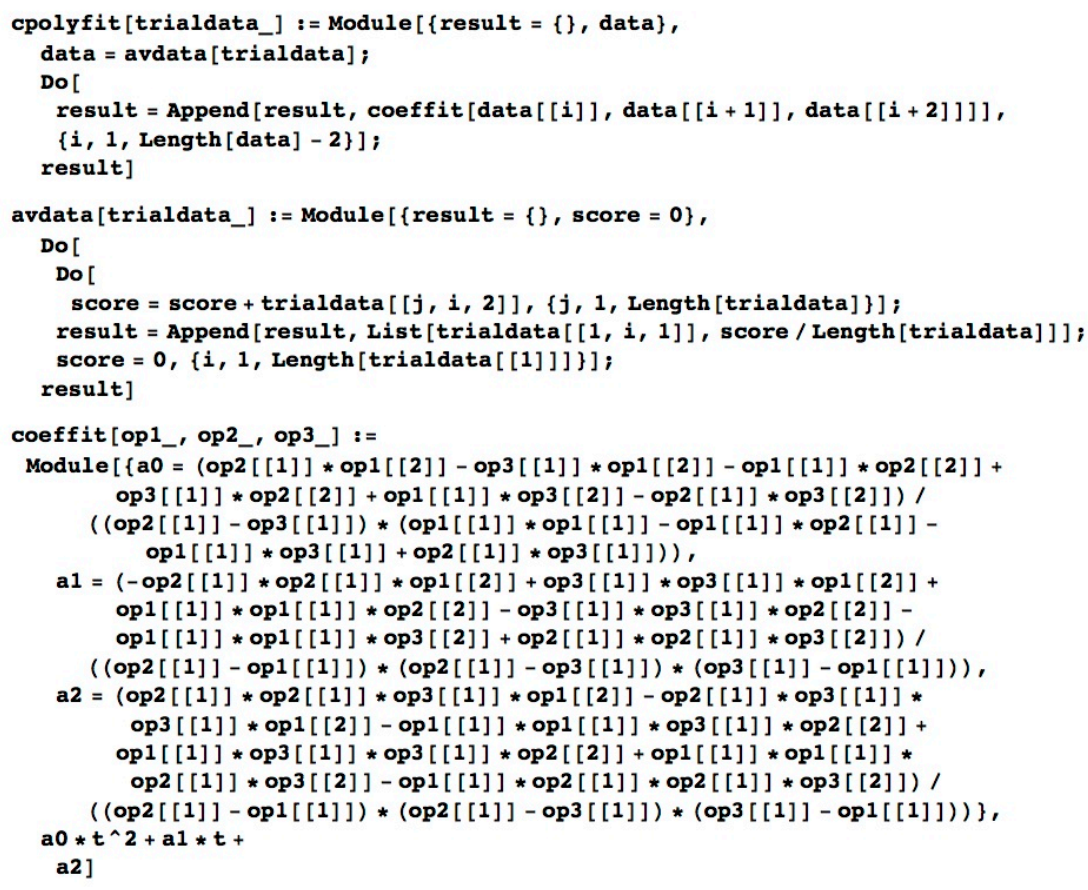

Calculation of Polynomial Sets for Each Variable

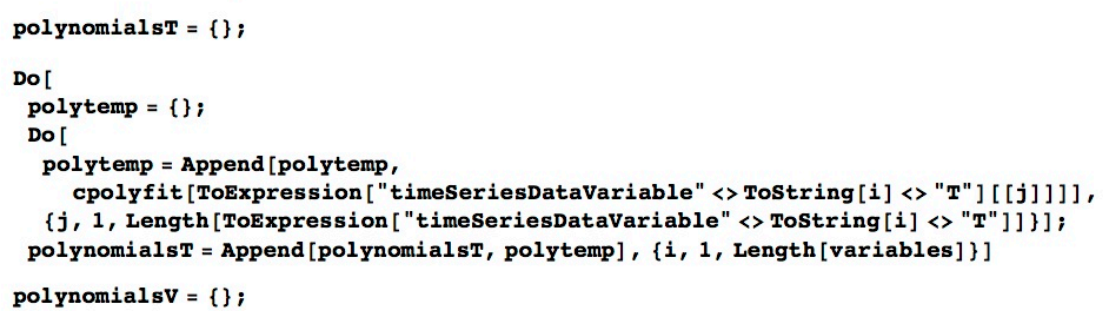




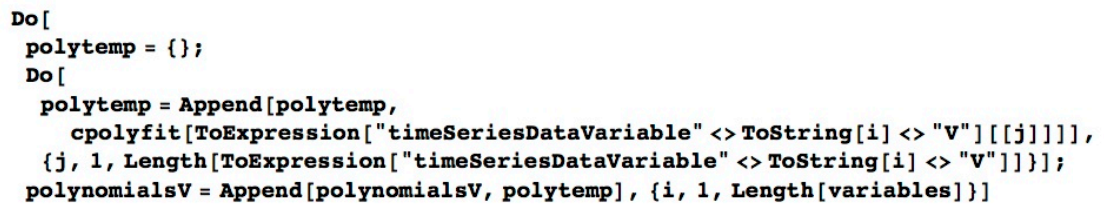

\section{Calculation of Sample Size}

This assumes that each trial of each condition includes the same number of experimental time points.

sampleSize = Length $[$ timeSeriesDataVariable1T $[1,1]]]$;

\section{Parameter Optimization}

The "parOpt" function will optimize a set of parameters for each candidate solution. For this single variable mass balance step, the best set of many randomly generated sets will be taken as the optimal parameter set.

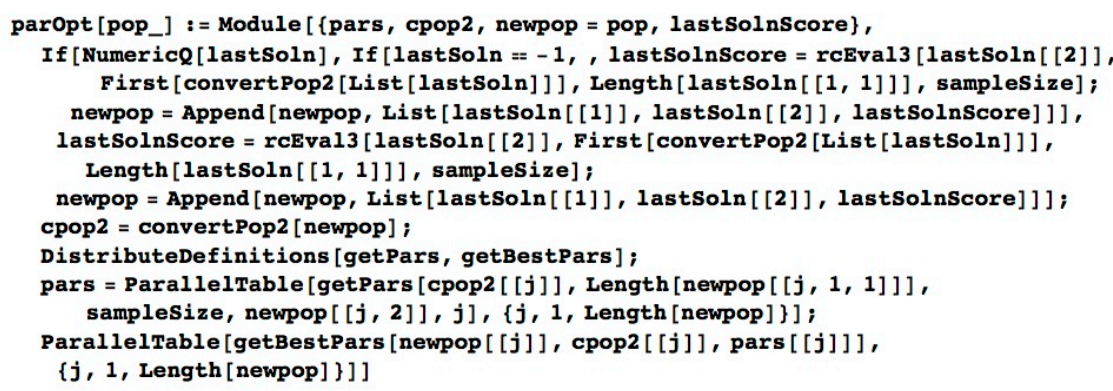

The following functions will translate a chromosome from the encoded form to a set of ordinary differential equations that can be used to directly calcuate derivative for comarison to data derivative estimates or that can be used for numerical integration and prediction of variable concentrations for direct comparison to experimental data (functions for this case end in " 2 ").

convertPop [pop_] : = Module $[\{$ result $=\{\}\}$,

Do $[$ result $=$ Append $[$ result, convertChrom [translateChrom [pop $[i, 1]]]]]$,

$\{i, 1$, Length [pop] $\}$; result $]$

convertPop2 [pop_] := Module $[\{$ result $=\{\}\}$,

Do $[$ result $=$ Append $[$ result, convertChrom2 $[$ trans lateChrom2 $[$ pop $[[i, 1]]]]]$,

$\{i, 1$, Length [pop] $\}]$; result ] 


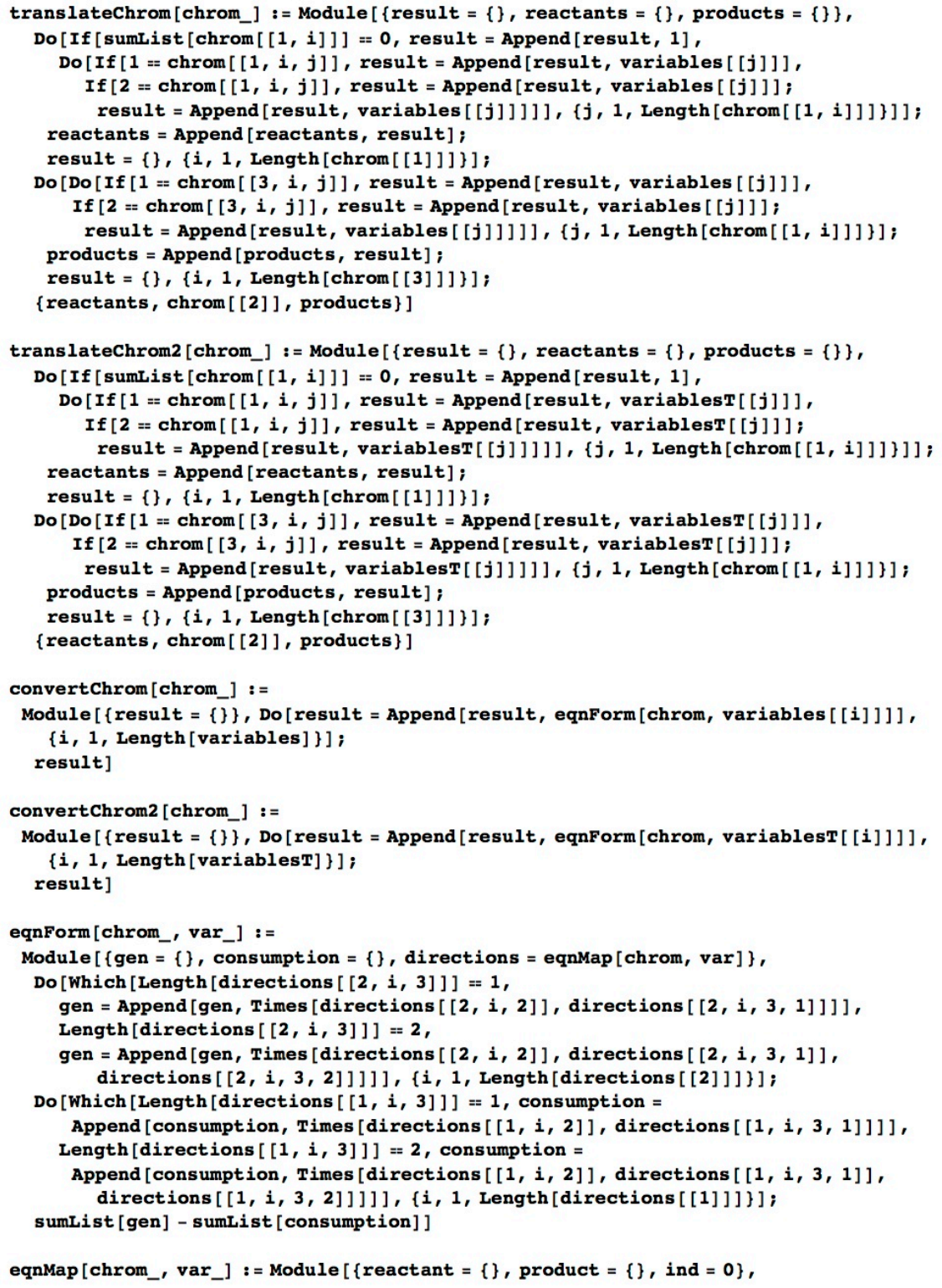




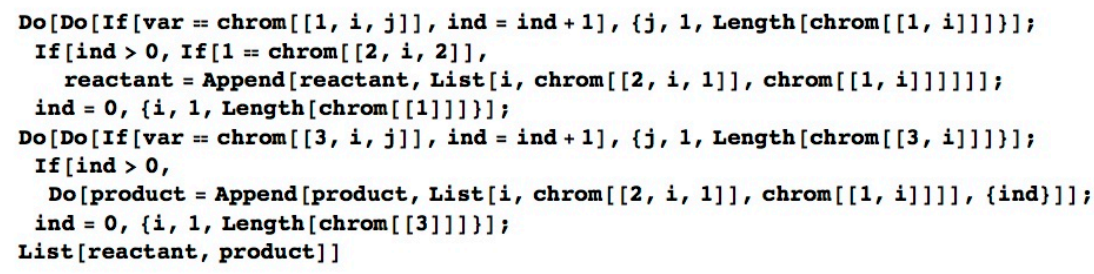

The "getBestPars" function will idenitfy the score of the parameter set on the validation initial condition and determine if it is better that then score of the parameter set from the previous iteration.

getBestPars [sprogram_, cprogram_, pars_] := Module [ $\{$ vscore $\}$ vscore $=\operatorname{rcEval} 3[$ pars $[[2]], \operatorname{cprogram}, \operatorname{Length}[\operatorname{sprogram}[[1,1]]]$, samplesize $]$; If [vscore < sprogram [ [3]], List [sprogram [1]], pars [2]], vscore], sprogram] ] 
The "rcEval2" function will evaluate the AICc score of a solution on the training data set by using numerical integration of the model.

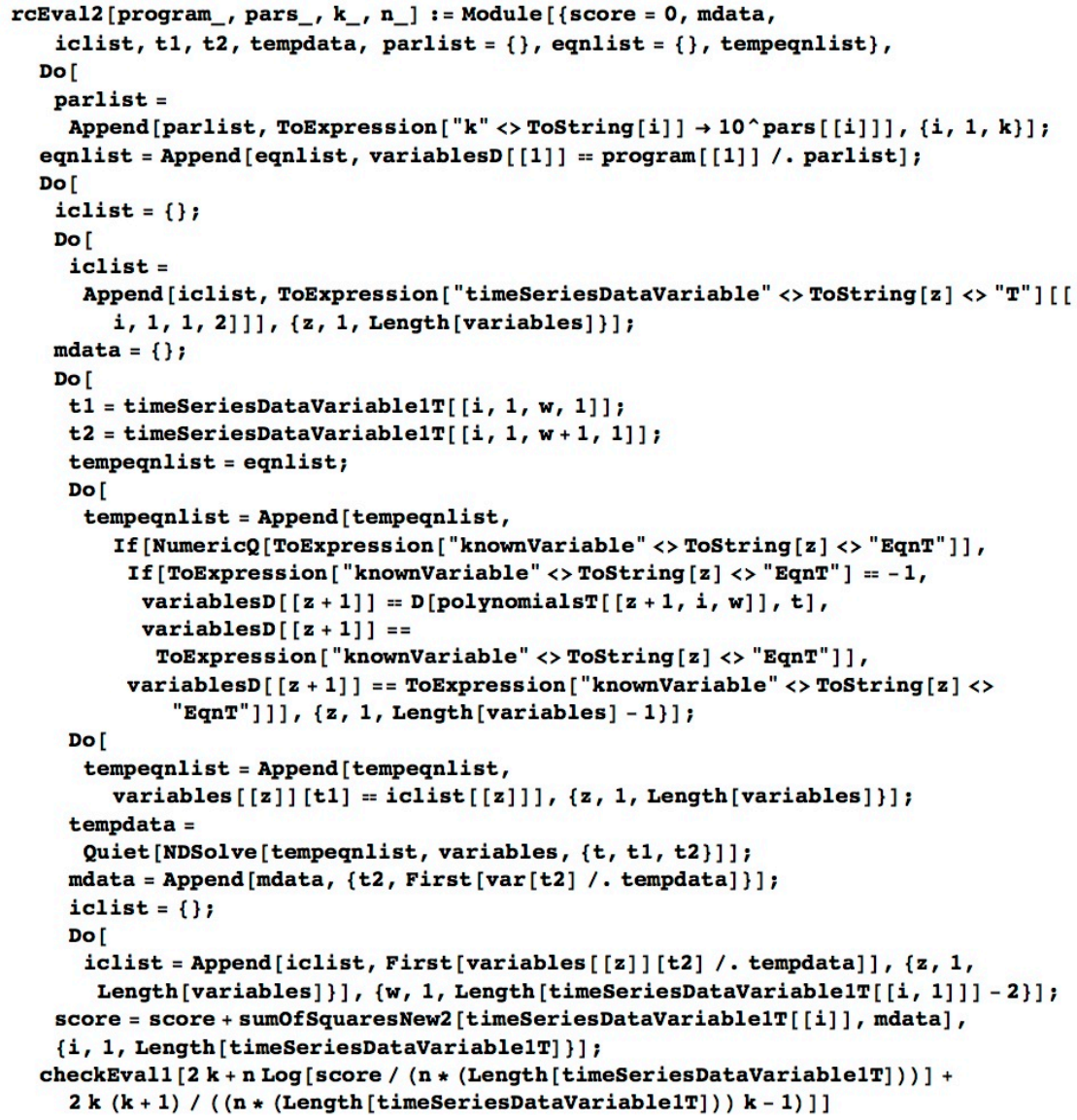


The "rcEval3" function will calculate the AICc score of a solution and its optimial parameters on the validation data set.

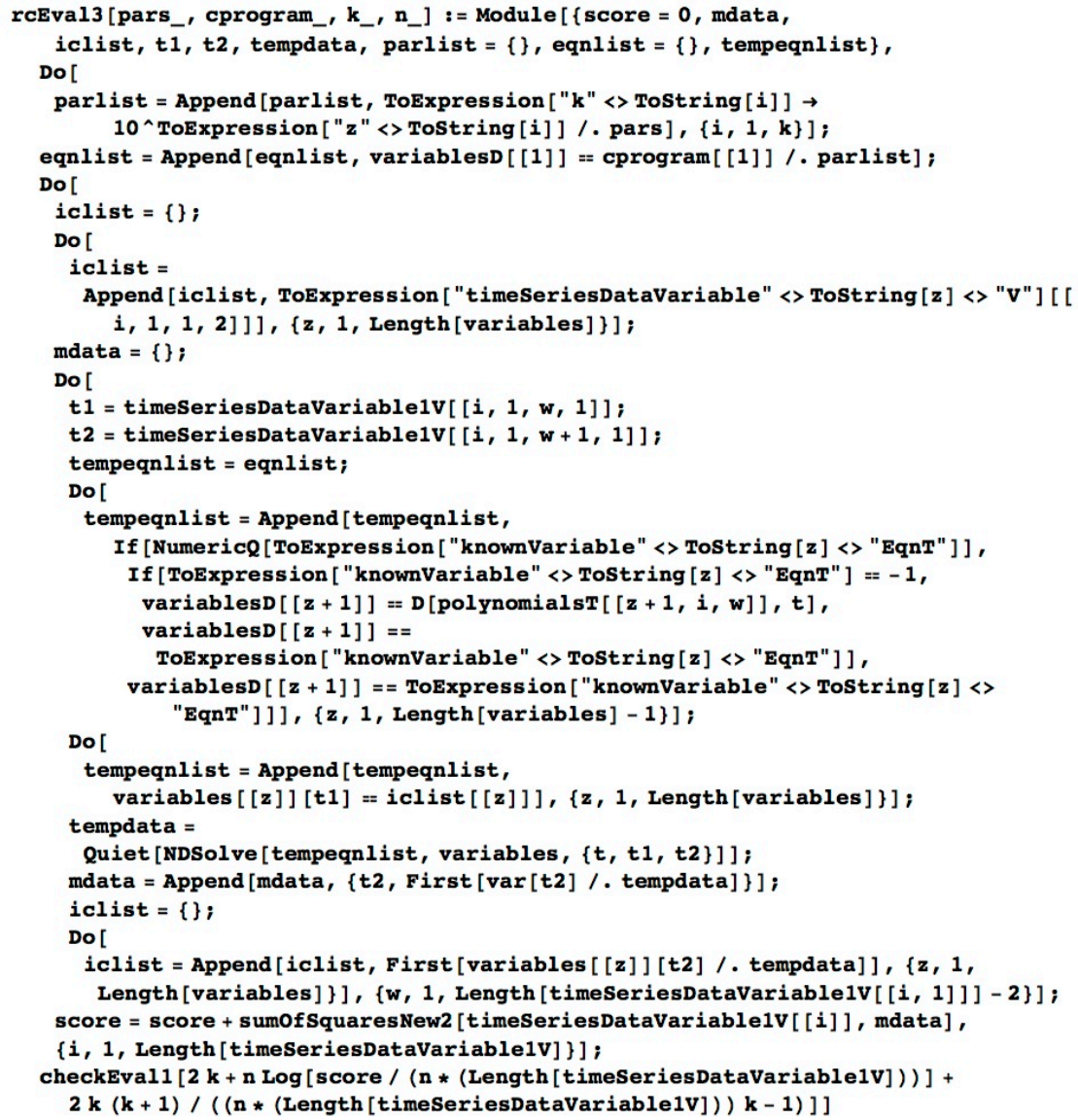

The "checkEvalI" function will replace any non-numerical score as a result of computational overflow with a very large score of $I^{*} 10^{\wedge}(100)$...this score will move a potential solution to the bottom of the population.

checkEval1 [evalResult_] : = If [NumericQ[evalResult], evalResult, $1 * 10^{\wedge} 100$ ]

The "sumOfSquaresNew2" function will calculate the sum of squared differences 
between model data and experimental data.

sumof SquaresNew2 [expdata_, mdata_] : =

Module $[\{\operatorname{sum}=0\}, \operatorname{Do}[\operatorname{Do}[\operatorname{sum}=\operatorname{sum}+(\operatorname{expdata}[[i, j+1,2]]-\operatorname{mdata}[[j, 2]]) \wedge 2$,

$\{j, 1$, Length [mdata $]\},\{i, 1$, Length [expdata $]\}]$; sum]

\section{Execution of the Algorithm}

The "sortPop" function will sort a population of potential solutions by score which is located in the 3 rd position of the chromosome.

sortPop[spop_] : = Sort [spop, \#1[[3]] $<\# 2[[3]] \&]$

The "sumList" function will return the summation of a vector or matrix.

sumList [1st_] := Module [ $\{$ sum $=0\}, \operatorname{Do}[$ If [VectorQ[1st [i]] ] ||MatrixQ[1st [[i]]], $\operatorname{sum}=\operatorname{sum}+\operatorname{sumList}[1 \mathrm{st}[[i]]], \operatorname{sum}=\operatorname{sum}+1$ st $[[i]]],\{i, 1$, Length $[1$ st $]\}] ;$ sum $]$

The command below will exectute the evolutionary algorithm to optimize a mass balance for the variable under consideration.

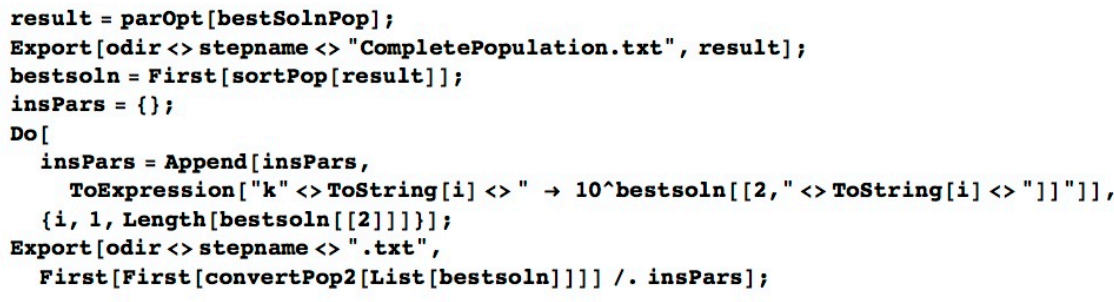




\subsubsection{EvolutionaryAlgorithm_SingleVariableStepComparisonOfMultipleInstancesWorkAround.nb}

This program is written to identify the best solution out of a number of instances run to evolve a single mass balance as part of the algorithm to evolve a complete mass action kinetic model. This program does more than just sort the solutions by score; the parameters associated with each solution are used as initital guesses to a numerical parameter optimization funtion. The solution with the best score after this secondary parameter optimization operation is taken as the newly evolved mass balance for the variable under investigation.

This notebook is to be used if EvolutionaryAlgorithm_SingleVariableStepComparisonOfMultiplelnstances program does not terminate due to failure of numerical parameter optimization runs to terminate. Please follow the instructions below using the output files from single solution numerical parameter optimization instances from the EvolutionaryAlgorithm_SingleVariableStepComparisonOfMultiplelnstances program.

Also, if the output containing the optimized balance contains " $z$ " terms, please elimiate the " $z->$ " expressions and evaluate the equation to get the correct condensed equation.

To run this program, please specify the necessary input and then evaluate the entire notebook by navigating to "Evaluation" and selecting "Evaluate Notebook".

\section{Input}

Much of this information can be directly copied from the single variable instances.

\section{Variable for which a mass balance is being evolved}

This needs to correspond to the first variable in the variables list below under System Information. Also, this variable should be considered to be Variable 1 when inputting time series data sets.

$\operatorname{var}=\mathbf{x} ;$

maxinteractions $=4$;

List of best solutions from each single variable mass balance instance for this step

bestSolnPop $=\{\} ;$

\section{Current solution (in interaction table form) for variable under investigation}

If this is the first iteration, define solution as "-1". MAKE SURE that parameters in interaction table are listed in $\left(\mathrm{z}^{*} \rightarrow \log\right.$ real num $)$ form.

lastSoln $=-1$;

\section{Parameters Numerically Evolved (taken from output files)}

Make sure that position in the list corresponds with the number associated with the output file. There will be one more element to this list then in the bestSolnPop as the last element corresponds to the parameters optimized for the lastSoln. If an output file does not exist for a solution, define parameters as "gpars" (generic parameters). 
parameters $=\{\}$

Output directory, please change to desired output location

odir = "/Users/homedir/";

If running multiple instances of the algorithm, assign a unique run identification to differentiate output

stepname = "VariableStep1BestofInstances";

System Information

variables $=\{\operatorname{var} 1, \operatorname{var} 2, \operatorname{var} 3\} ;$ (* variables involved in

the model for which a time series data set has been collected,

this representation is used for calculations involving derivative estimates *)

$\operatorname{variables} T=\{\operatorname{var} 1[t], \operatorname{var} 2[t], \operatorname{var} 3[t]\} ;(*$ variables involved

in the model for which a time series data set has been collected,

this representation is used for numerical integration calculations *)

variablesD $=\left\{\operatorname{var} 1{ }^{\prime}[t], \operatorname{var2}{ }^{\prime}[t], \operatorname{var} 3^{\prime}[t]\right\}$;

(* first order derivatives for all variables *)

List of Estimated Steady States for Each Variable

$\operatorname{var} 1 \mathbf{s s}=\mathbf{x} ;$

$\operatorname{var2SS}=\mathbf{Y} ;$

$\operatorname{var} 3 \mathbf{S S}=\mathbf{z} ;$

Mass balances for other species

If a mass balance has not yet been evolved for a given variable, recored the knownVariableEqn (used for calculations involving derivarive estimates) and knownVariableEqnT (used for calculations involving numerical integration) as "-1".

Note: Variable 1 below is not the same as the variable for which a mass balance is being evolved since the assumption of this algorithm is that the equation for said variable is considered unknown.

knownVariable1Eqn $=-1$;

knownVariable1EqnT $=-1$

knownVariable2Eqn $=-1$;

knownVariable2EqnT = - 1;

(* Continue for any other known variables *)

\section{Data ( $T$ = Training Data Set, V = Validation Data Set)}

Enter as $\{\{\{\{$ time1, conc1\}, \{time2, varconc2\}...\}\}\} where the brackets represent \{entire data set $\},\{\{$ data set for an initial condition\}...\}, \{\{\{trial for an initial conition\}... ...\},\{\{\{timepoints, concdata\}...\}......\}

Note: Variable numbers should correspond to variable order in the list of variables under System Informa tion above. 


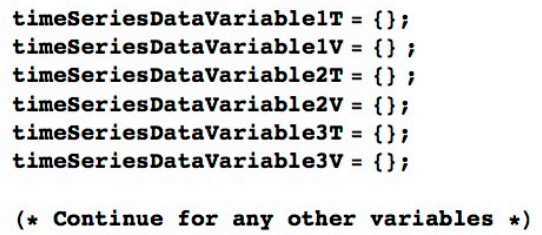

Make Data and Equations Available Across All Nodes (for parallelization tasks)

List all data sets, known equations, and steady states here so that they are availble to all nodes, MAKE SURE TO CHANGE NAMES AS CHANGED ABOVE!

DistributeDefinitions [timeSeriesDataVariable1T, timeSeriesDataVariable1v, timeSeriesDataVariable2T, timeSeriesDataVariable2V, timeSeriesDatavariable3T, timeSeriesDataVariable3v, knownVariable1Eqn, knownVariable1EqnT, knownVariable2Eqn, knownVariable2EqnT, var1sS, var2SS, var3SS];

\section{Data Preparation}

The "cpolyfit", "avdata", and "coeffit" functions are used to fit polynomials to each three consecutive data points. These polynomials can be used to estimate variable values at intermediate time points as well as to estimate derivatives at points of interest.

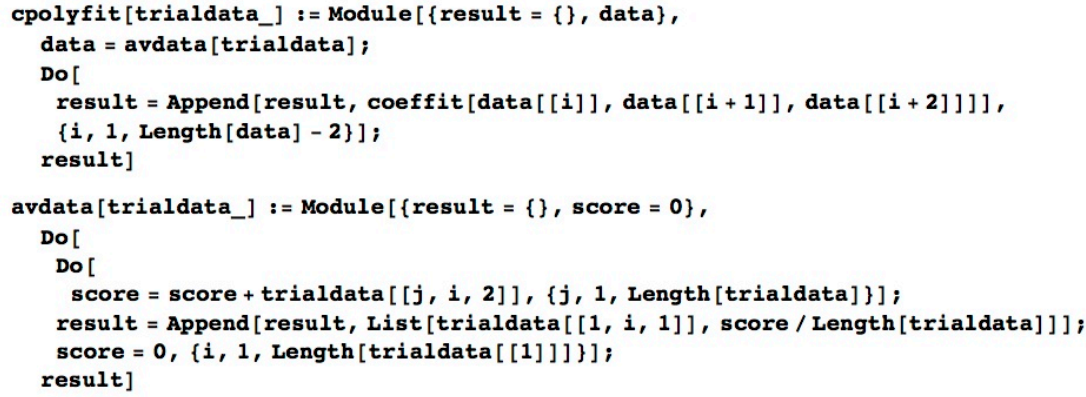




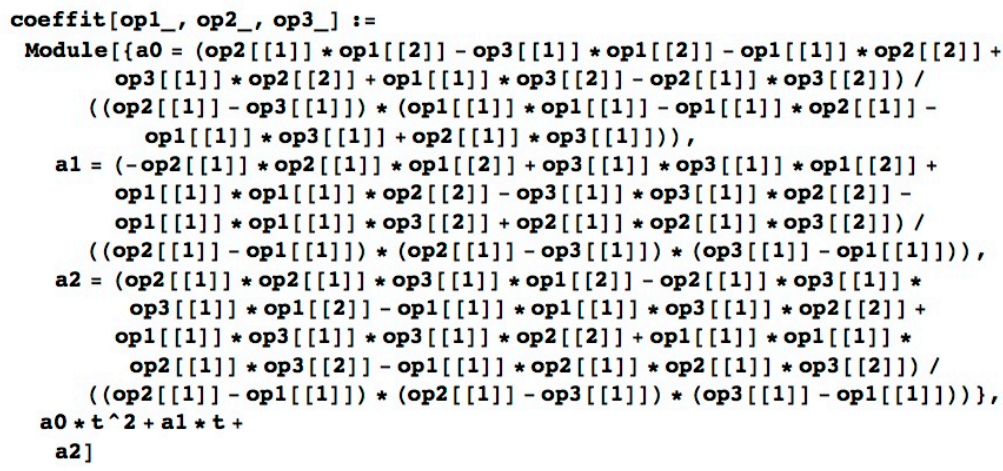

Calculation of Polynomial Sets for Each Variable

polynomialst $=\{\} ;$

Do [

polytemp $=\{\} ;$

Do [

polytemp = Append [polytemp,

cpolyfit [ToExpression ["timeSeriesDataVariable" <> ToString[i] <> "T"] [j]]]],

$\{j, 1$, Length [ToExpression [ timeSeriesDataVariable" $<>T o S t r i n g[i]<>~ " T "]]\}]$;

polynomialsT $=$ Append [polynomialsT, polytemp],$\{i, 1$, Length [variables $]\}]$

polynomialsv $=\{\}$;

Do [

polytemp $=\{\} ;$

Do [

polytemp = Append [polytemp

cpolyfit [ToExpression ["timeSeriesDataVariable" $<$ ToString [i] 〈> "v"] [j]]]],

$\{j, 1$, Length [ToExpression [ timeSeriesDataVariable" $<$ ToString [i] <> "v"]] ] ;

polynomialsv $=$ Append [polynomialsv, polytemp $],\{i, 1$, Length $[$ variables $]\}]$

Generation of Generic Parameters (will be eliminated in favor of original parameters)

gpars = Table [ToExpression $[" z "\langle>$ ToString $[i]\langle>" \rightarrow-200 "],\{i, 1$, maxInteractions $\}]$

$\{z 1 \rightarrow-200, z 2 \rightarrow-200, z 3 \rightarrow-200, z 4 \rightarrow-200\}$

\section{Calculation of Sample Size}

This assumes that each trial of each condition includes the same number of experimental time points.

sampleSize $=$ Length $[$ timeSeriesDataVariable1T $[1,1]]]$; 


\section{Parameter Optimization}

The "parOpt" function will optimize a set of parameters for each candidate solution. For this single variable mass balance step, the best set of many randomly generated sets will be taken as the optimal parameter set.

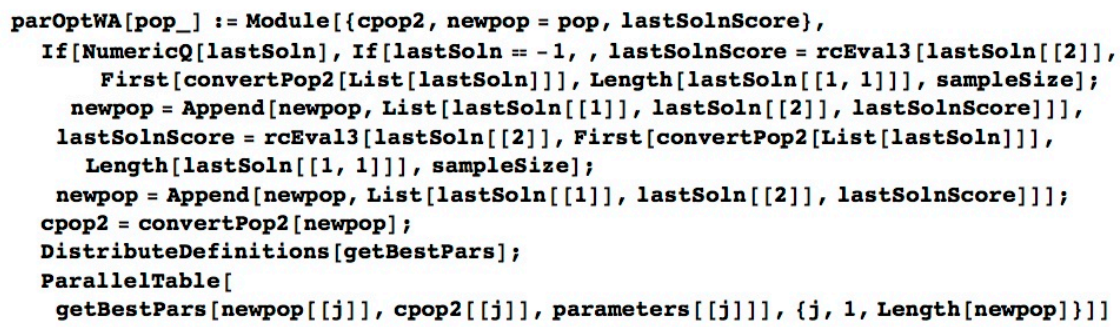

The following functions will translate a chromosome from the encoded form to a set of ordinary differential equations that can be used to directly calcuate derivative for comarison to data derivative estimates or that can be used for numerical integration and prediction of variable concentrations for direct comparison to experimental data (functions for this case end in " 2 ").

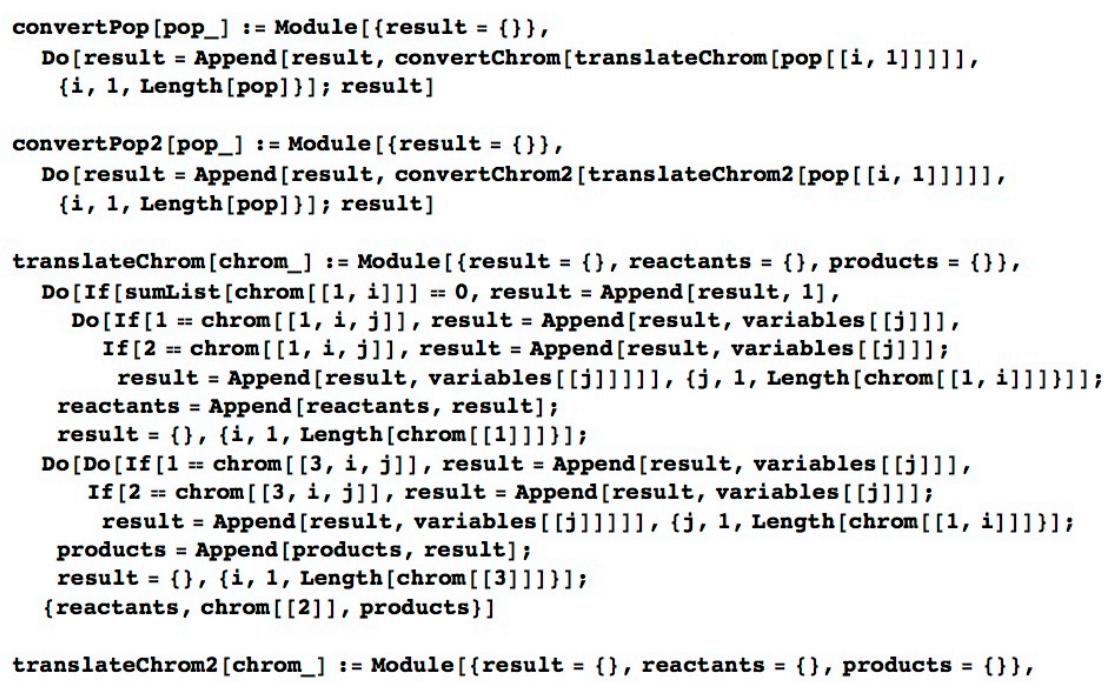




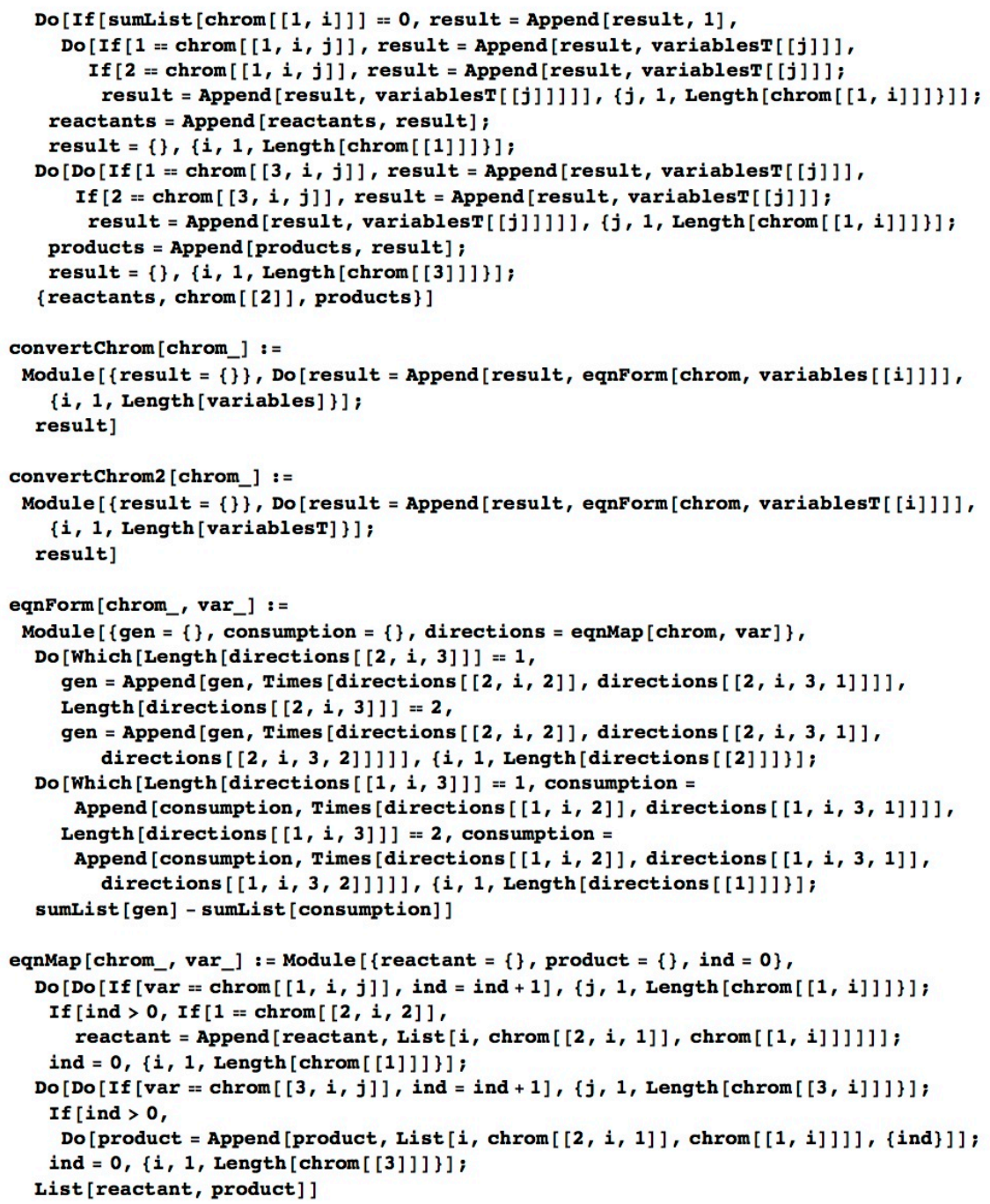

The "getBestPars" function will idenitfy the score of the parameter set on the validation initial condition and determine if it is better that then score of the parameter set from the previous iteration. 
getBestPars [sprogram_, cprogram_, pars_] := Module [ $\{$ vscore $\}$,

vscore $=\operatorname{rcEval} 3[$ pars, $\operatorname{cprogram}, \operatorname{Length}[\operatorname{sprogram}[[1,1]]]$, samplesize $] ;$

If [vscore < sprogram [3]], List [sprogram [11] , pars, vscore], sprogram] ]

The "rcEval2" function will evaluate the AICc score of a solution on the training data set by using numerical integration of the model.

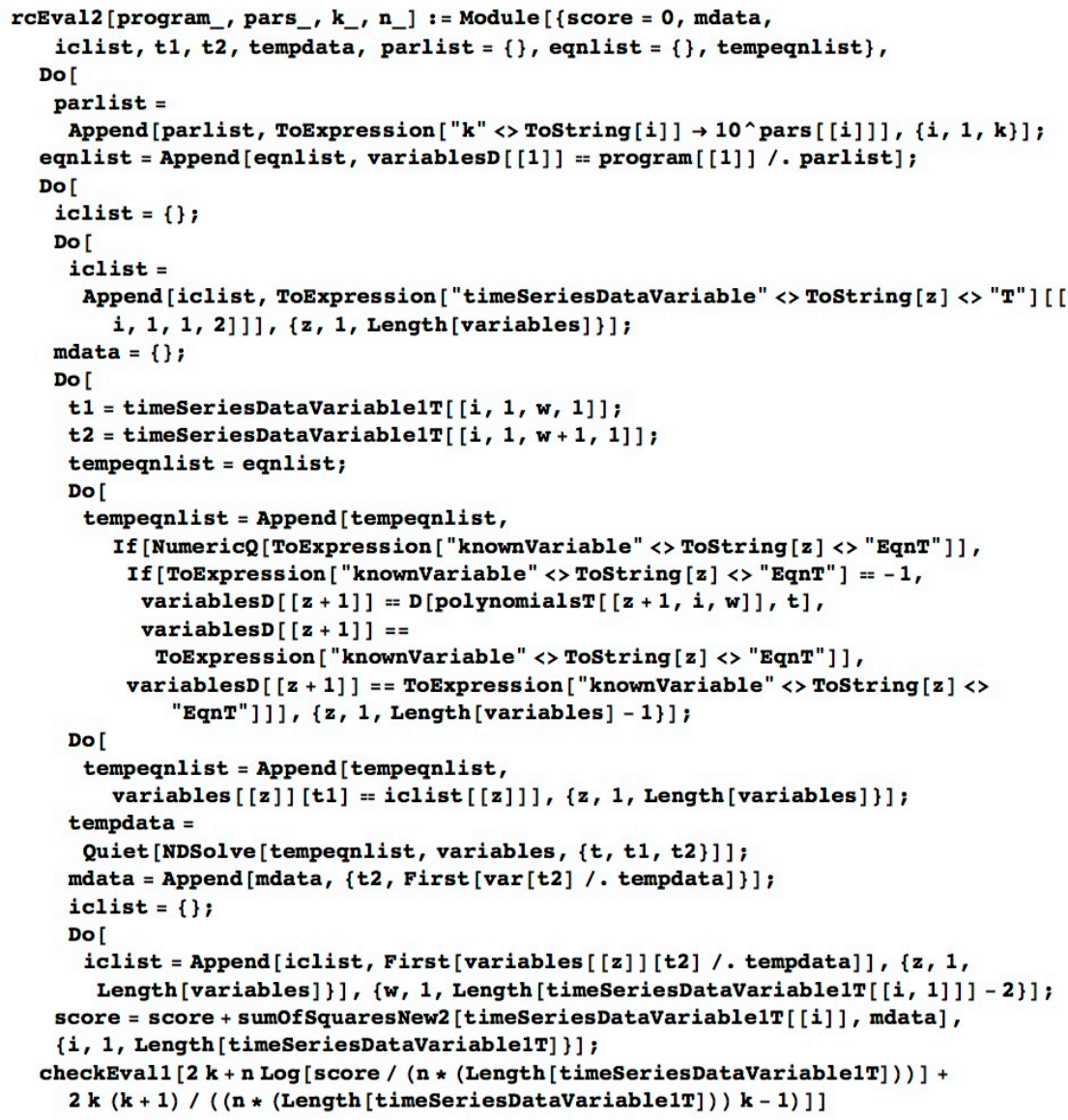


The "rcEval3" function will calculate the AICc score of a solution and its optimial parameters on the validation data set.

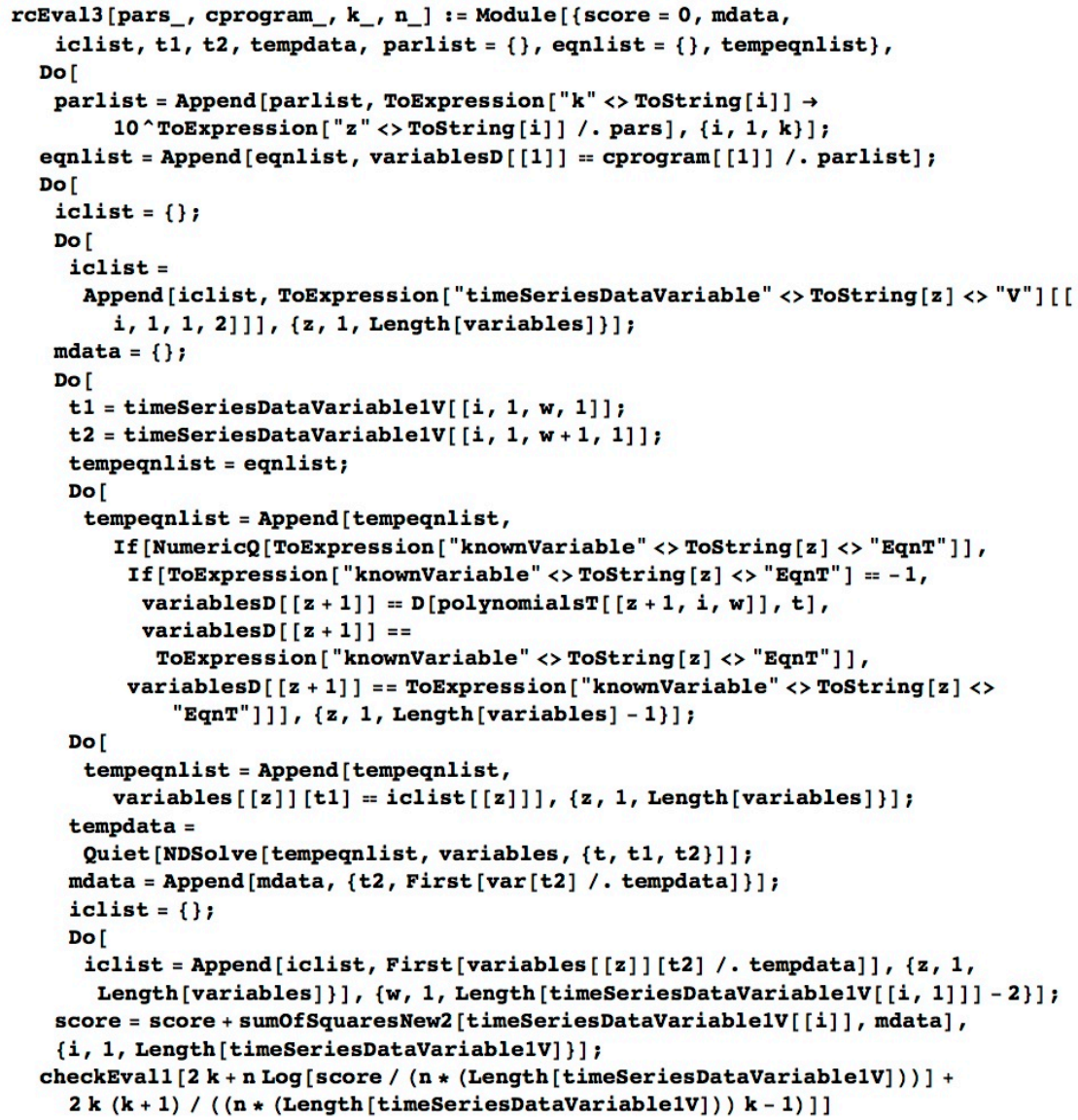

The "checkEvalI" function will replace any non-numerical score as a result of computational overflow with a very large score of $I^{*} 10^{\wedge}(100)$...this score will move a potential solution to the bottom of the population.

checkEval1 [evalResult_] : = If [NumericQ[evalResult], evalResult, $1 * 10^{\wedge} 100$ ]

The "sumOfSquaresNew2" function will calculate the sum of squared differences 
between model data and experimental data.

sumof SquaresNew2 [expdata_, mdata_] : =

Module $[\{\operatorname{sum}=0\}, \operatorname{Do}[\operatorname{Do}[\operatorname{sum}=\operatorname{sum}+(\operatorname{expdata}[[i, j+1,2]]-\operatorname{mdata}[[j, 2]]) \wedge 2$,

$\{j, 1$, Length $[$ mdata $]\}],\{i, 1$, Length [expdata $]\}] ;$ sum]

\section{Execution of the Algorithm}

The "sortPop" function will sort a population of potential solutions by score which is located in the 3 rd position of the chromosome.

sortPop[spop_] : = Sort [spop, \#1 [ [3]] $<\# 2[[3]] \&]$

The command below will exectute the evolutionary algorithm to optimize a mass balance for the variable under consideration.

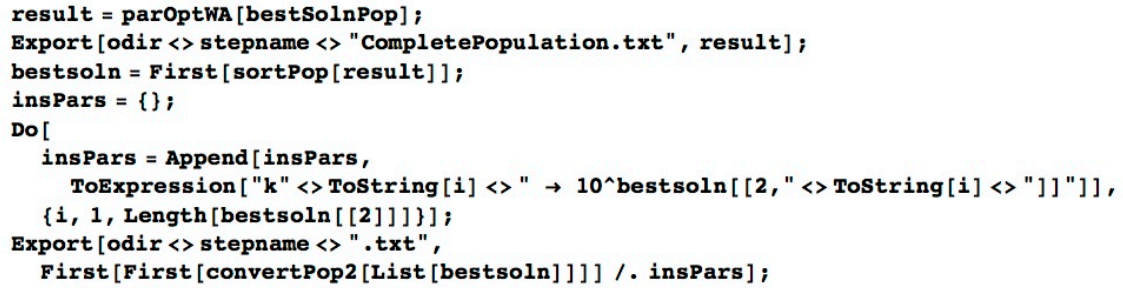




\subsubsection{EvolutionaryAlgorithm_CompleteModelOptimization(FinalStep).nb}

This program is written to simultaneously evolve a set of equations as the final step in the evolution of a mass action kinetic model from an experimental data set.

To run this program, please specify the necessary input and then evaluate the entire notebook by navigating to "Evaluation" and selecting "Evaluate Notebook".

\section{Input}

Output directory, please change to desired output location

$$
\text { odir = "/Users/homedir/"; }
$$

If running multiple instances of the algorithm, assign a unique run identification to differentiate output

$$
\begin{aligned}
& \begin{array}{l}
\text { stepname = "Finalstep" ; } \\
\text { run = "a"; }
\end{array} \\
& \text { Evolutionary Parameters }
\end{aligned}
$$

\section{Evolutionary Parameters}

popsize $=30 ;(*$ population size $*)$

generations $=150 ;(*$ number of generations over which

the algorithm will execute, MUST BE A MULTIPLE OF 10 *)

recombinationPercentages $=\{90,80,70,60,50,40,30,20,10,0\}$;

(* percentage of solutions recombining,

as changed over the evolution of the population $*$ )

mutationPercentages $=\{5,10,15,20,25,30,35,40,45,50\}$;

(* percentage of solutions mutating,

as changed over the evolution of the population *)

\section{System Information}

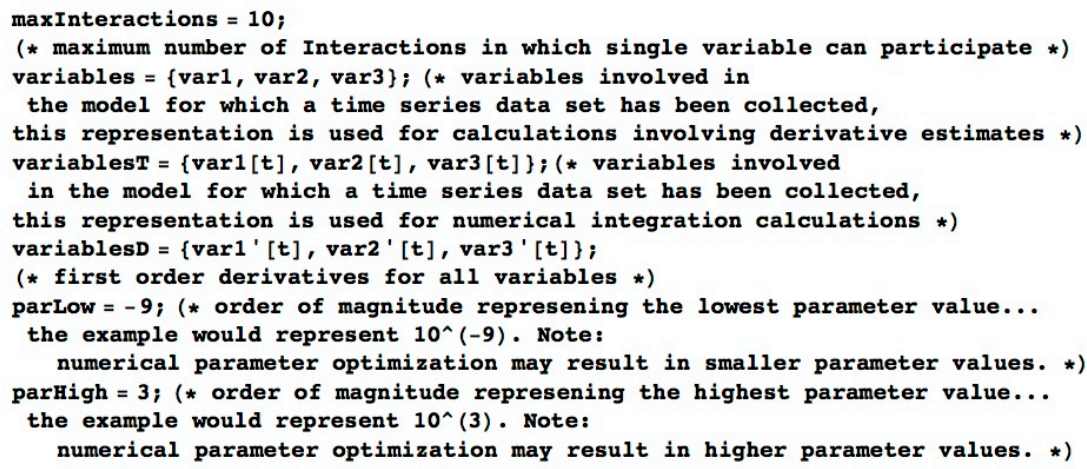


List of Estimated Steady States for Each Variable

$\operatorname{var1ss}=\mathbf{x}$;

$\operatorname{var2sS}=\mathbf{y} ;$

$\operatorname{var3ss}=\mathbf{z} ;$

\section{Seed Interactions}

List the reactants, rate constants, and products of interactions hypothesized by completing the single variable steps of the algorithm. These interactions will be used to seed the final population of potential models. The position in each vector should match up with the reactants, rate constants, and products for an interaction and the number of positions in each reactant or product allele should equal the number of variables in the system (shown for a system of 3 variables). The list can be as long as the maxinteractions input but it is recommended that fewer interactions be specified allowing for diversity in the initial population of models.

seedReactants $=\{\} ;$

seedParameters $=\{\}$;

seedProducts $=\{\}$;

Data ( $T$ = Training Data Set, V = Validation Data Set)

Enter as $\{\{\{\{t i m e 1$, conc1\}, \{time2, varconc2\}...\}\}\} where the brackets represent \{entire data set\}, $\{$ data set for an initial condition\}...\}, \{\{\{trial for an initial conition\}...\}...,\{\{\{\{timepoints, concdata\}...\}......\}

Note: Variable numbers should correspond to variable order in the list of variables under System Informa tion above.

timeSeriesDataVariable1T $=\{\}$;

timeSeriesDataVariable1v $=\{\}$

timeSeriesDataVariable2T $=\{\}$;

timeSeriesDataVariable2v $=\{\}$;

timeSeriesDataVariable3T $=\{\} ;$

timeSeriesDataVariable3v $=\{\}$;

(* Continue for any other variables *)

Define Functions That Are Dependant on the Maximum Number Of Interactions

The two functions below need to be definied in terms of the number of interactions under investigation. Please follow the directions in the ( ${ }^{*}$ comments $\left.{ }^{*}\right)$ to definie the functions correctly. The functions are currently set up for a maximum of 10 interactions. 
The "getPars" function will calculate the model score on the training initial conditions. The best five parameter sets from the derivative estimate calculation are used as the initial guesses to a parameter optimization function.

getPars [program_, $\mathbf{k}_{-}$, pars_] : $=(*$ List $\mathbf{z} 1 \rightarrow$

$\mathrm{zN}$ are arguments to NMinimize where "N" is the maximum number of interactions*) NMinimize [rcEvalPRE2 [program, z1, z2, z3, z4, z5, z6, z7, z8, z9, z10, k, sampleSize]

( $*$ Define bounds $\{\{z 1, \operatorname{pars}[[1]]-1, \operatorname{pars}[[1]]+1\} \rightarrow\{\mathrm{zN}, \operatorname{pars}[[N]]-1, \operatorname{pars}[[N]]+1\}$ where $" \mathrm{~N} "$ is the maximum number of interactions*)

$\{\{z 1, \operatorname{checkPar}[\operatorname{pars}[[1]]]-1, \operatorname{checkPar}[\operatorname{pars}[[1]]]+1\}$

$\{z 2, \operatorname{checkPar}[\operatorname{pars}[[2]]]-1, \operatorname{checkPar}[\operatorname{pars}[[2]]]+1\}$

$\{z 3, \operatorname{checkPar}[\operatorname{pars}[[3]]]-1, \operatorname{checkPar}[\operatorname{pars}[[3]]]+1\}$

$\{z 4$, checkPar [pars [ [4]]]-1, checkPar [pars [ [4]]] +1$\}$,

$\{z 5$, checkPar $[$ pars $[[5]]]-1, \operatorname{checkPar}[\operatorname{pars}[[5]]]+1\}$,

$\{z 6, \operatorname{checkPar}[\operatorname{pars}[[6]]]-1, \operatorname{checkPar}[\operatorname{pars}[[6]]]+1\}$

$\{z 7, \operatorname{checkPar}[\operatorname{pars}[[7]]]-1, \operatorname{checkPar}[\operatorname{pars}[[7]]]+1\}$,

$\{z 8, \operatorname{checkPar}[\operatorname{pars}[[8]]]-1, \operatorname{checkPar}[\operatorname{pars}[[8]]]+1\}$,

$\{z 9, \operatorname{checkPar}[\operatorname{pars}[[9]]]-1, \operatorname{checkPar}[\operatorname{pars}[[9]]]+1\}$,

$\{z 10$, checkPar [pars [[10] ] $]-1, \operatorname{checkPar}[\operatorname{pars}[[10]]]+1\}\}]$

checkPar [par_] := If $[$ NumericQ $[$ par $]$, par, -100$]$

(* List $\mathrm{z} 1$ NumericQ $\rightarrow \mathrm{zN}$ NumericQ as arguments to rCEvalPRE2 where " $N "$ is the maximum number of interactions *)

rCEvalPRE2 [program_, z1_? NumericQ, z2_? NumericQ, z3_? NumericQ,

$\mathrm{z4}$ 4 NumericQ, z5_? NumericQ, z6_? NumericQ, z7_? NumericQ,

z8_? NumericQ, z9_? NumericQ, z10_? NumericQ, k_, n_] :=

(* List List $[\mathrm{z} 1 \rightarrow \mathrm{zN}]$ as arguments to rcEval2 where

"N" is the maximum number of interactions *)

reEval2 [program, List $[\mathrm{z} 1, \mathrm{z2}, \mathrm{z3}, \mathrm{z4}, \mathrm{z5}, \mathrm{z6}, \mathrm{z7}, \mathrm{z8}, \mathrm{z9}, \mathrm{z10}], \mathrm{k}, \mathrm{n}$ ]

Make Data and Equations Available Across All Nodes (for parallelization tasks)

List all data sets, known equations, and steady states here so that they are availble to all nodes

DistributeDefinitions [timeSeriesDataVariable1T, timeSeriesDataVariable1v,

timeSeriesDataVariable2T, timeSeriesDataVariable2V,

timeSeriesDataVariable3T, timeSeriesDataVariable3v, var1ss, var2ss, var3ss] ; 


\section{Data Preparation}

The "cpolyfit", "avdata", and "coeffit" functions are used to fit polynomials to each three consecutive data points. These polynomials can be used to estimate variable values at intermediate time points as well as to estimate derivatives at points of interest.

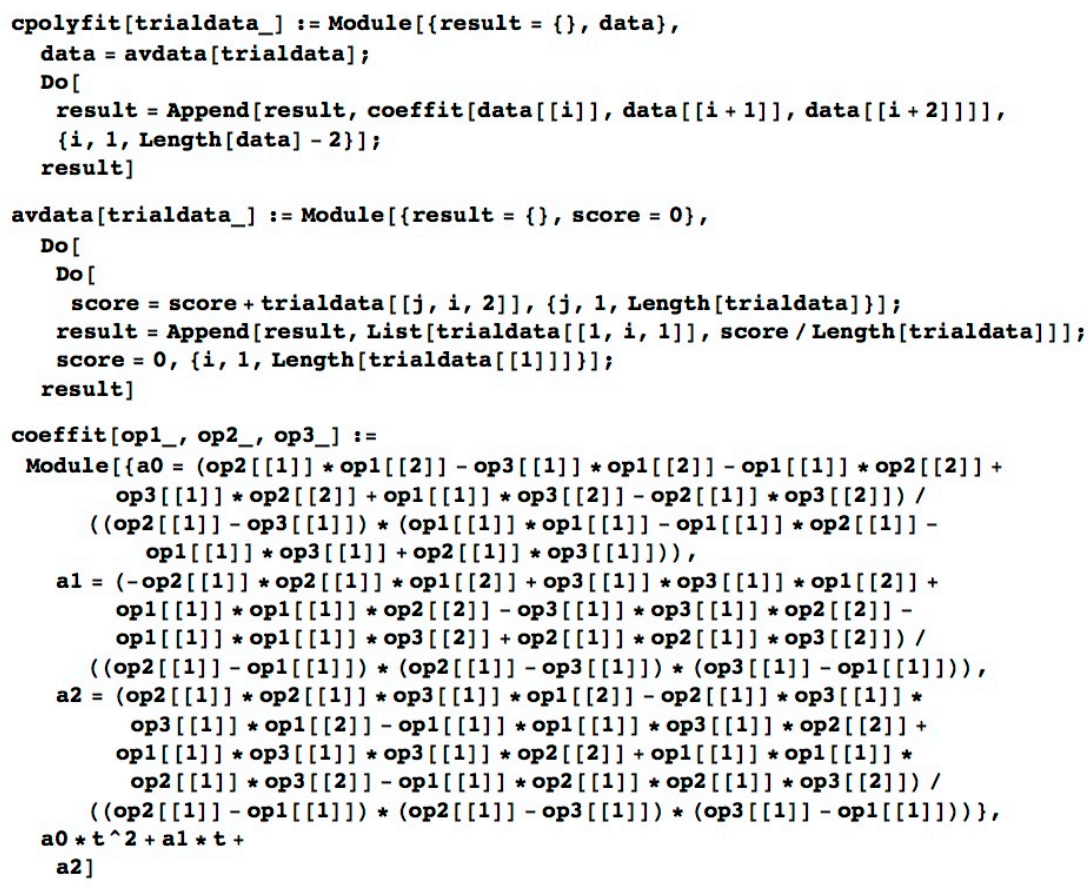

Calculation of Polynomial Sets for Each Variable

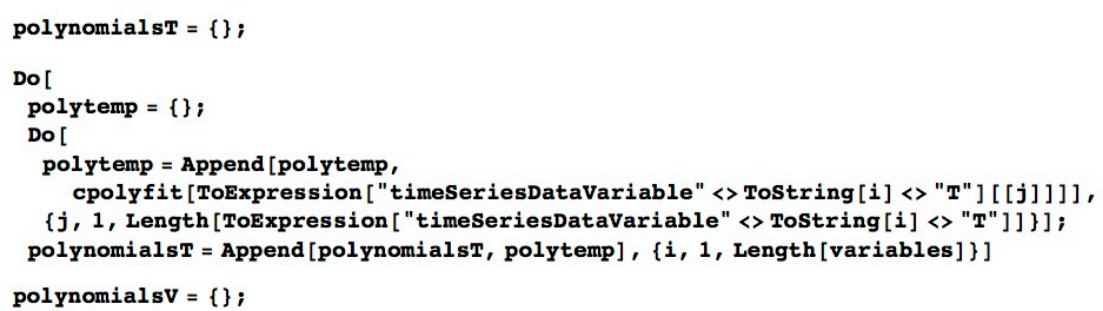




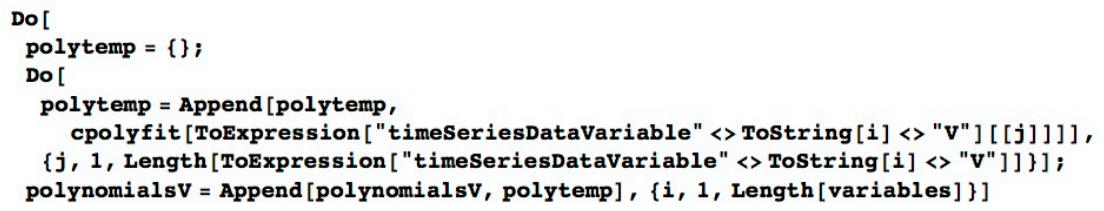

Calculation of Derivative Estimates for Each Variable at Each Experimental Time Point (needed only for the training data set)

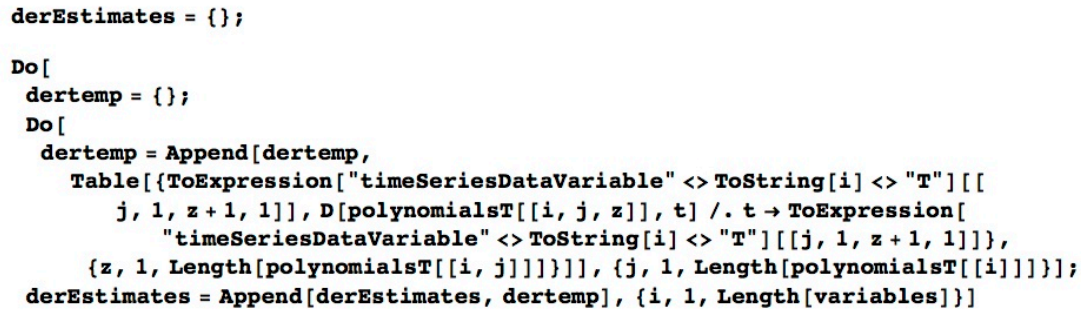

\section{Calculation of Sample Size}

This assumes that each trial of each condition includes the same number of experimental time points.

sampleSize $=$ Length $[$ timeSeriesDataVariable1T $[[1,1]]]$;

\section{Evolutionary Operations}

The "ea" function is the top level function for the execution of the evolutionary algorithm. A population of potential solutions will be generated and will undergo cycles of parameter optimization, selection, recombination, and mutation until the desired number of generation has been fulfilled.

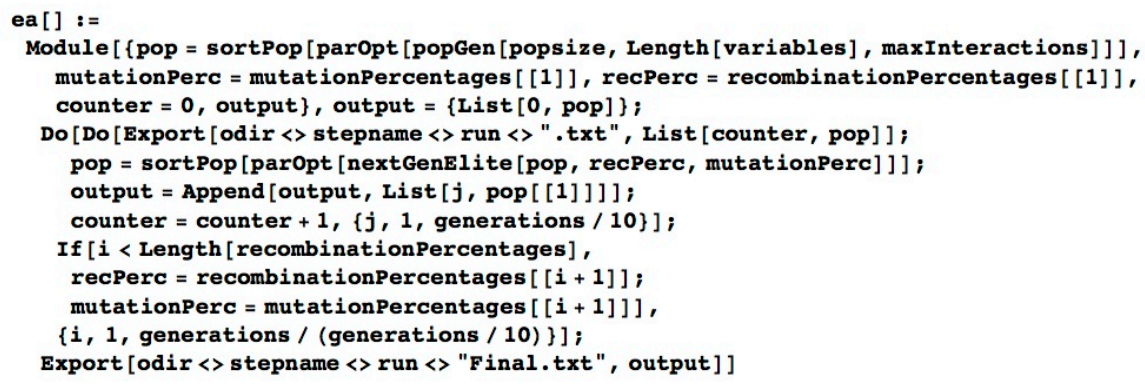


The "rc" function will generate a random parameter on a log scale within the range specified.

rc []$:=$ RandomReal $[\{$ parLow, parHigh $\}]$

The "popGen" function will generate an initial population of potential solutions using the specified population size, the number of variables in the system, and the maximum interactions for which the variable can be involved. The variable mass balance under analysis will be initiated with a zeroth order production term and a first order degradation term.

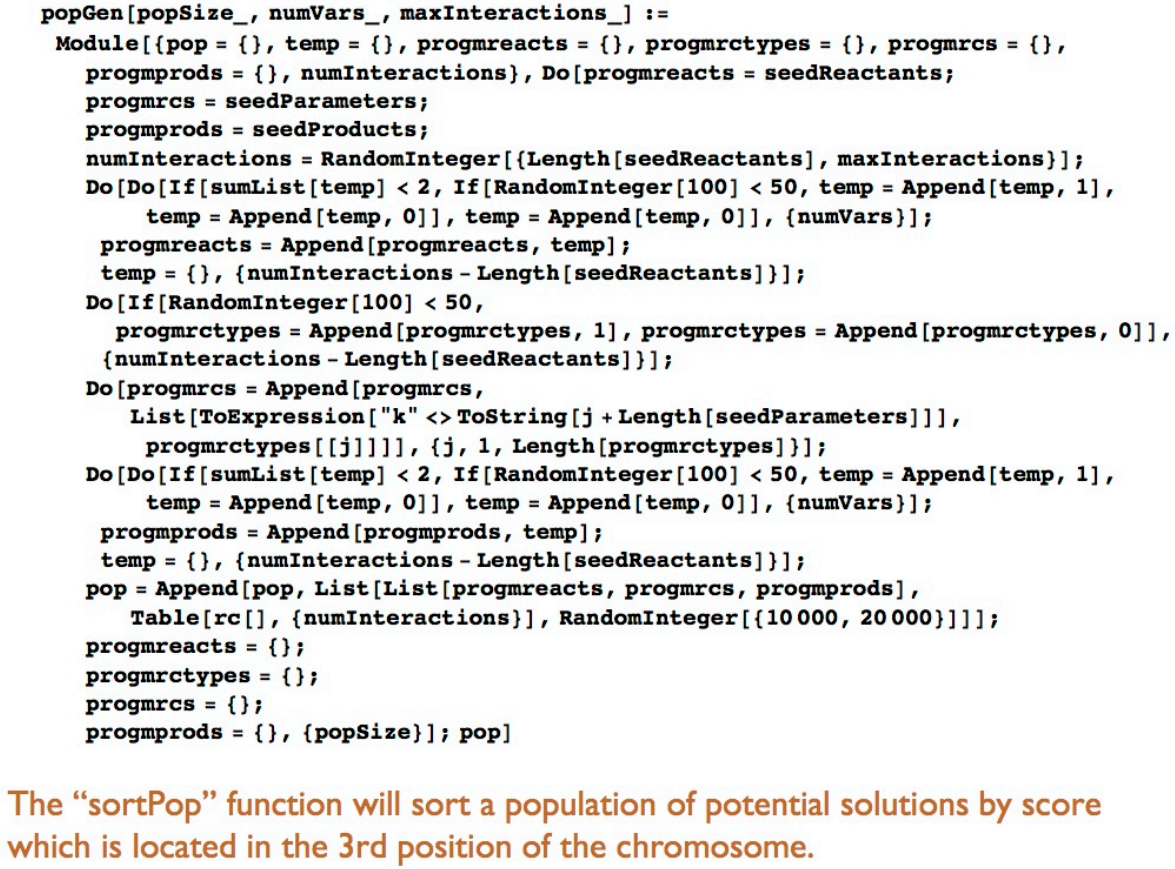

The "sortPop" function will sort a population of potential solutions by score which is located in the 3rd position of the chromosome.

sortPop[spop_] := Sort [spop, \#1[[3]]<\#2[[3]]\&] 
The "nextGenElite" function will carry out selection, recombination, and mutation operations to populate a next generation population of solutions that contains some old solutions and some newly generated solutions. The Elite selection strategy will be used.

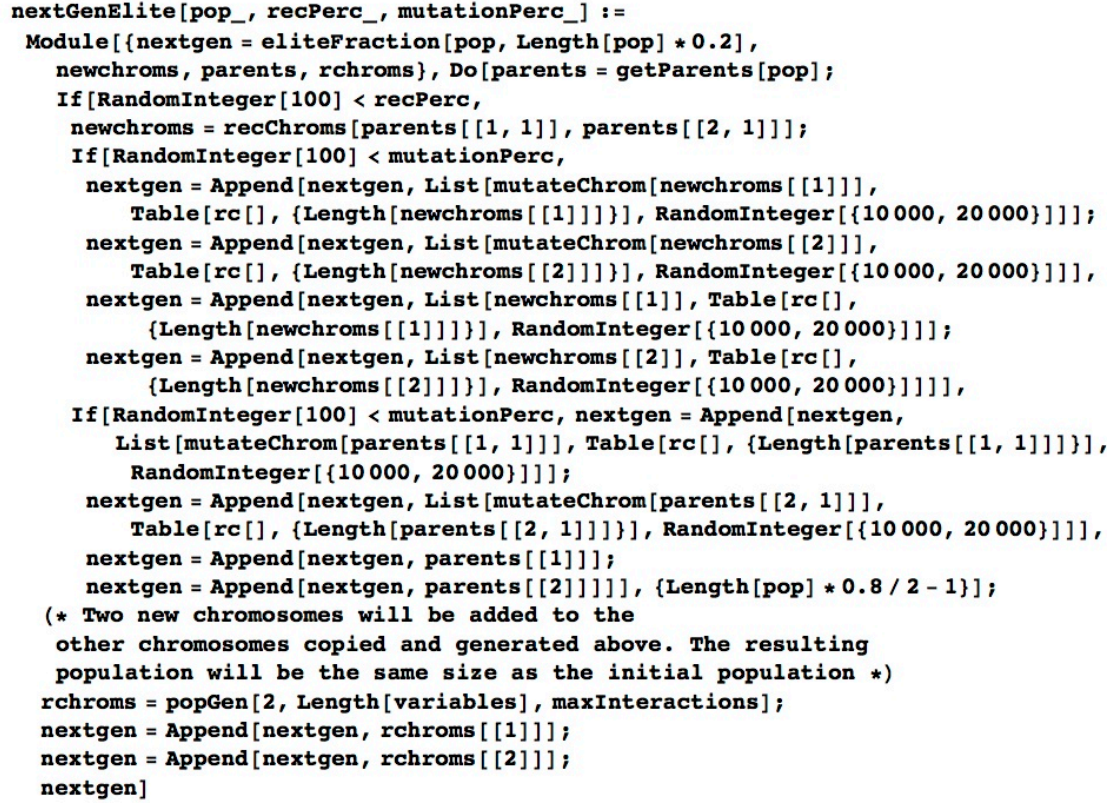

The "eliteFraction" function will take a percentage of the best scoring solution and output them to be copied unaltered to the next generation population of solutions.

eliteFraction [pop_, fractsize_] :=

Module $[\{$ result $=\{\}\}$, Do $[$ result $=$ Append $[$ result, pop $[i]]],\{i, 1$, fractsize $\}]$; result]

The "getParents" function ensures that two unique parents are selected for recombination.

getParents [pop_] : = Module $[\{$ parent $1=\operatorname{pop}[[\operatorname{RandomInteger}[\{1, \operatorname{Length}[$ pop $]\}]]]$, parent $2=\operatorname{pop}[[$ RandomInteger $[\{1$, Length $[$ pop $]\}]]]\}$, while [

parent1 [[3]] $=\operatorname{parent2}[[3]], \operatorname{parent2}=\operatorname{pop}[[\operatorname{RandomInteger}[\{1, \operatorname{Length}[$ pop $]\}]]]] ;$ \{parent1, parent2\} ] 
The "recChroms" function will recombine two chromosomes or solutions to form two new solutions.

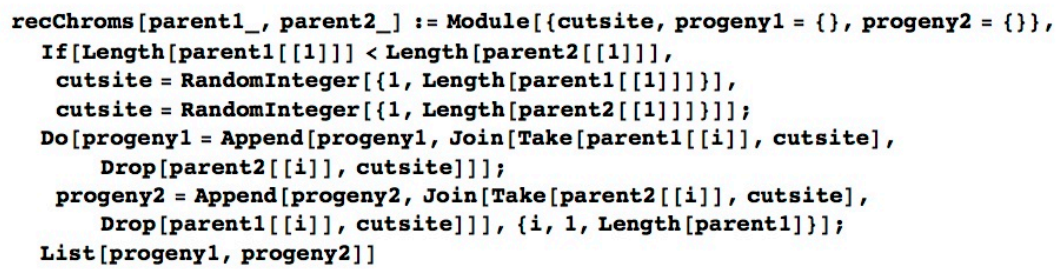

The "mutateChrom" function will make a point mutation in a solution.

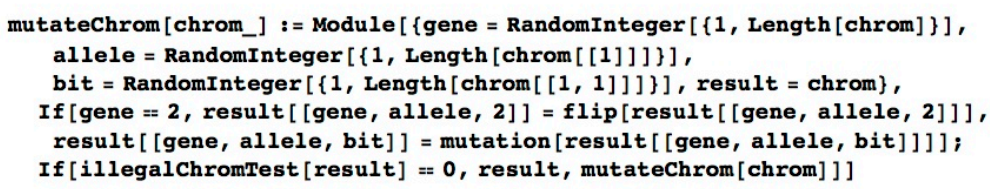

The "illegalChromTest" function will test to make sure that a point mutation does not result in an invalid chromosome that has more that 2 reactants or products.

illegalChromTest [chrom_] : = Module [ $\{$ result $=0\}$, $\operatorname{Do}[\operatorname{If}[\operatorname{sumList}[\operatorname{chrom}[[1, i]]]>2, \operatorname{result}=\operatorname{result}+1],\{i, 1, \operatorname{Length}[\operatorname{chrom}[[1]]]\}]$; Do $[$ If $[\operatorname{sumList}[\operatorname{chrom}[[3, i]]]>2$, result $=\operatorname{result}+1],\{i, 1, \operatorname{Length}[\operatorname{chrom}[[3]]]\}]$; result]

The "sumList" function will return the summation of a vector or matrix.

sumList [1st_] := Module $[\{$ sum $=0\}, \operatorname{Do}[$ If [VectorQ[1st [i] ] ] | MatrixQ[1st [i] ] ], sum $=\operatorname{sum}+\operatorname{sumList}[1 \mathrm{st}[[i]]], \operatorname{sum}=\operatorname{sum}+1 \mathrm{st}[[i]]],\{i, 1$, Length $[1 \mathrm{st}]\}]$; sum $]$

The "flip" function will mutate a "I" to a "0" or a "0" to a "I".

flip [bit_] := If $[$ bit $=1,0,1]$

The "mutation" will mutate a "0" to a "I" or "2", a "I" to a "0" or "2", or a "2" to a "0" or "I".

mutation [num_] : = Module $[\{$ new $=\operatorname{RandomInteger}[2]\}$, If $[$ num $==$ new, mutation $[$ num $]$, new $]]$

\section{Parameter Optimization}


The "parOpt" function will optimize a set of parameters for each candidate solution. For this single variable mass balance step, the best set of many randomly generated sets will be taken as the optimal parameter set.

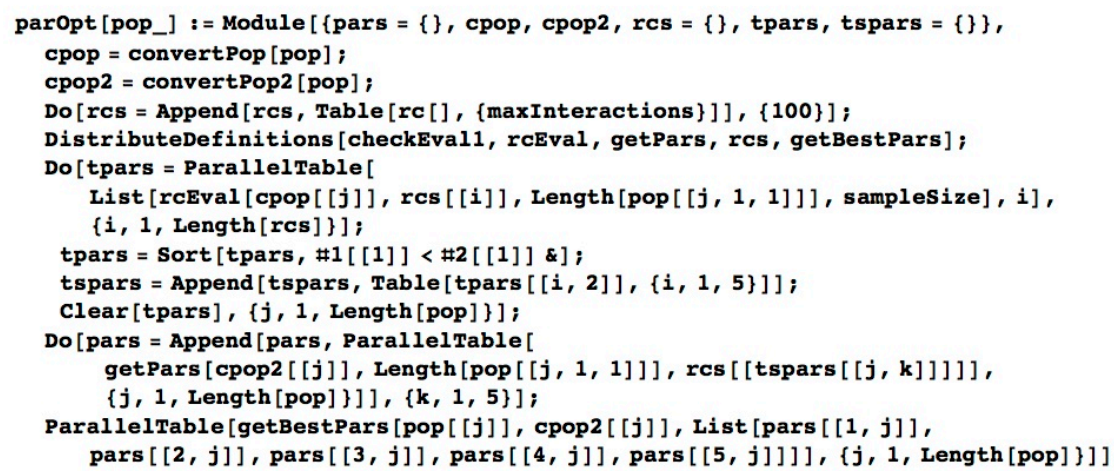

The following functions will translate a chromosome from the encoded form to a set of ordinary differential equations that can be used to directly calcuate derivative for comarison to data derivative estimates or that can be used for numerical integration and prediction of variable concentrations for direct comparison to experimental data (functions for this case end in " 2 ").

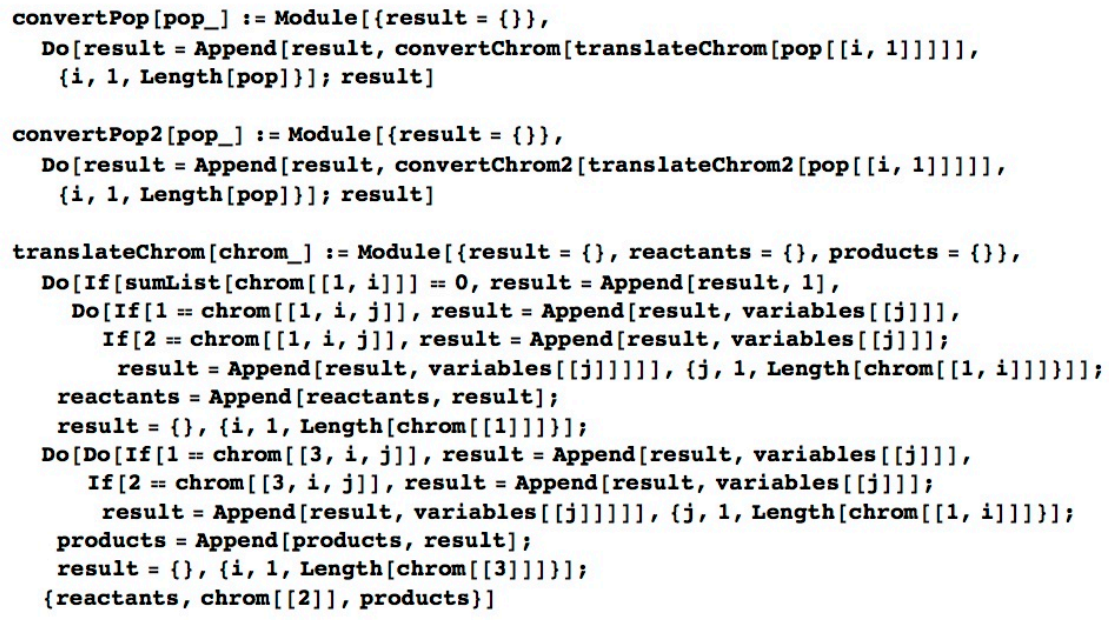




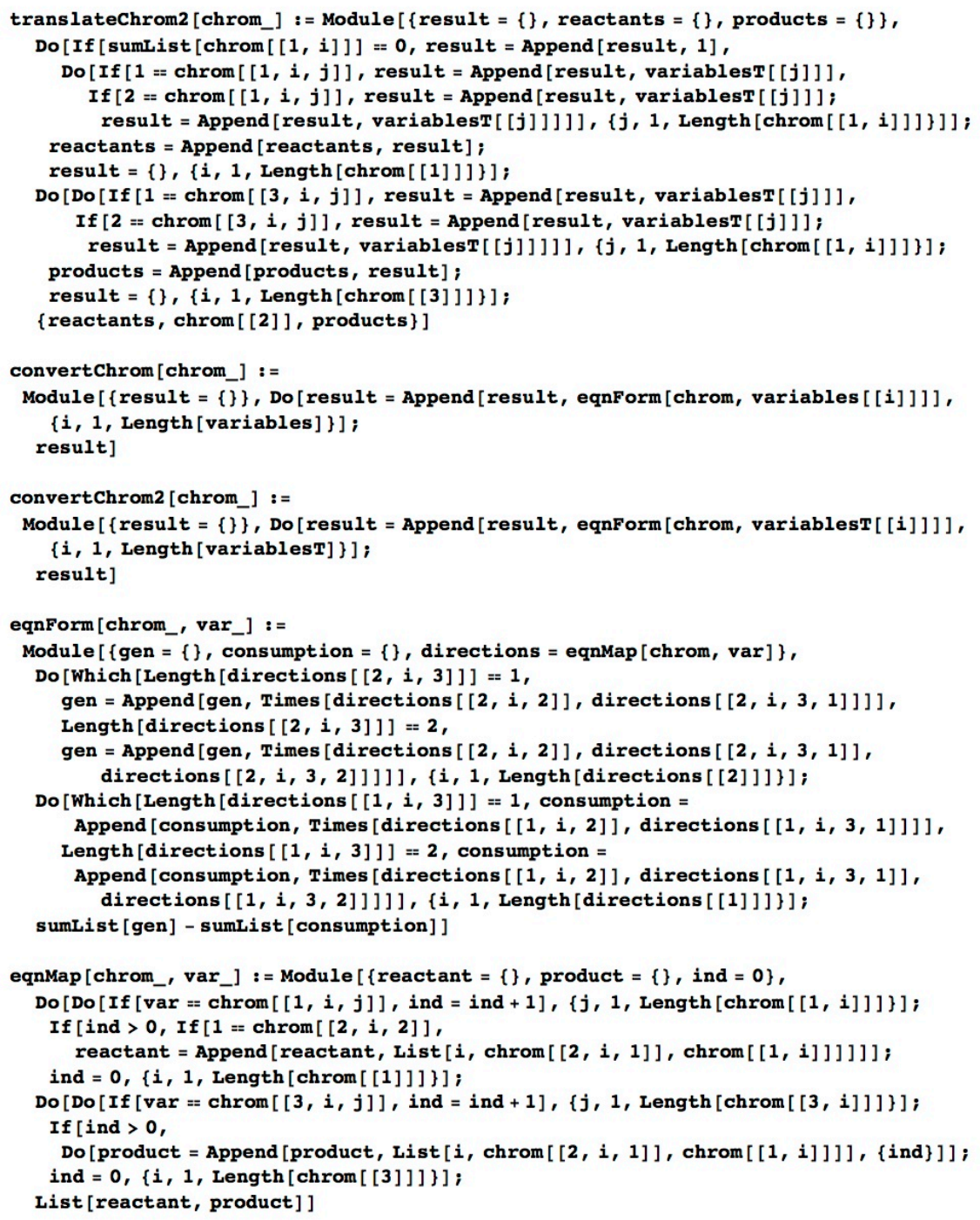

The "getBestPars" function will idenitfy the score of the parameter set on the validation initial condition and determine if it is better that then score of the parameter set from the previous iteration. 
getBestPars [sprogram_, cprogram_, pars_] :=

Module $[\{$ bpar, vscore $\}$, bpar $=$ First [Sort [pars, \#1 [[1]]<\#2[[1]]\&]];

vscore $=\operatorname{rcEval} 3[$ bpar $[[2]]$, cprogram, Length $[$ sprogram $[[1,1]]]$, sampleSize $]$;

If [vscore < sprogram [3]], List [sprogram [1]], bpar [2]], vscore], sprogram] ]

The "rcEval" function will evaluate the AICc score of a solution on the training data set by using derivative estimates.

rcEval [program_, pars_, $\left.k_{-}, n_{-}\right]:=\operatorname{Module}[\{\operatorname{score}=0, \operatorname{parlist}=\{\}$, eqnlist $=\{\}\}$, Do $[$ parlist $=$

Append [parlist, ToExpression ["k" $<>$ ToString [i]] $\rightarrow 10^{\wedge}$ pars [i]]], $\left.\{i, 1, k\}\right]$

Do [eqnlist = Append [eqnlist, program [i] ] / parlist] , $\{i, 1$, Length [variables] $\}]$;

Do $[$ score $=$ score + sumofSquaresNew [eqnlist, $i]$,

$\{i, 1$, Length [timeSeriesDataVariable1T] $\}]$;

checkEval1 [2 k + n Log [score / (n * (Length [timeSeriesDatavariable1T])) ] +

$2 \mathbf{k}(\mathbf{k}+1) /((\mathbf{n} *($ Length [timeSeriesDataVariable1T] $))-\mathbf{k}-1)]]$ 
The "rcEval2" function will evaluate the AICc score of a solution on the training data set by using numerical integration of the model.

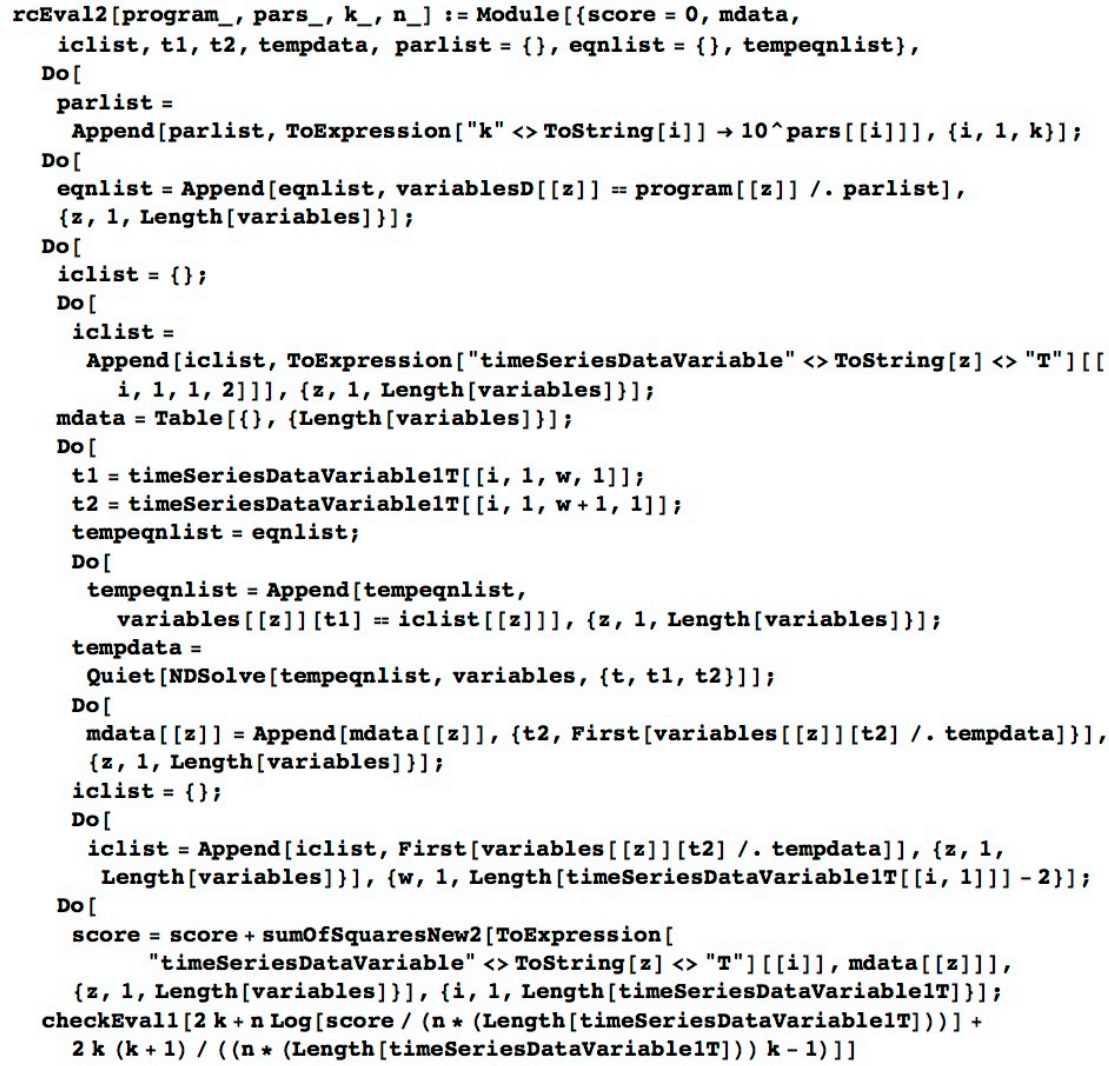


The "rcEval3" function will calculate the AICc score of a solution and its optimial parameters on the validation data set.

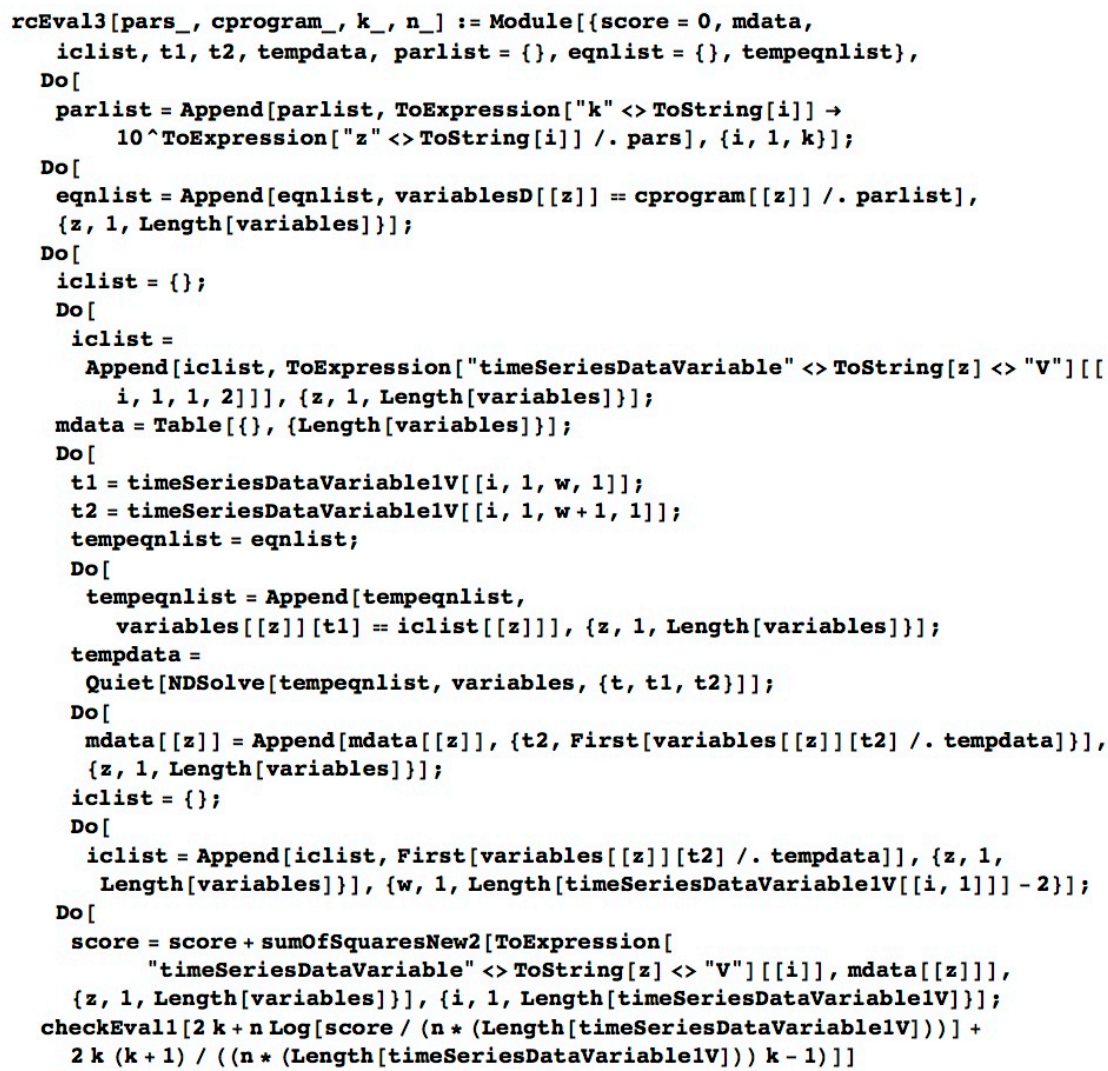

The "checkEvalI" function will replace any non-numerical score as a result of computational overflow with a very large score of $I^{*} \mid 0^{\wedge}(100)$...this score will move a potential solution to the bottom of the population.

checkEval1 [evalResult_] : = If [NumericQ[evalResult], evalResult, $1 * 10^{\wedge} 100$ ] 
The "sumOfSquaresNew" function will calculate the sum of squared differences between model derivate calculations and experimental data derivative estimates.

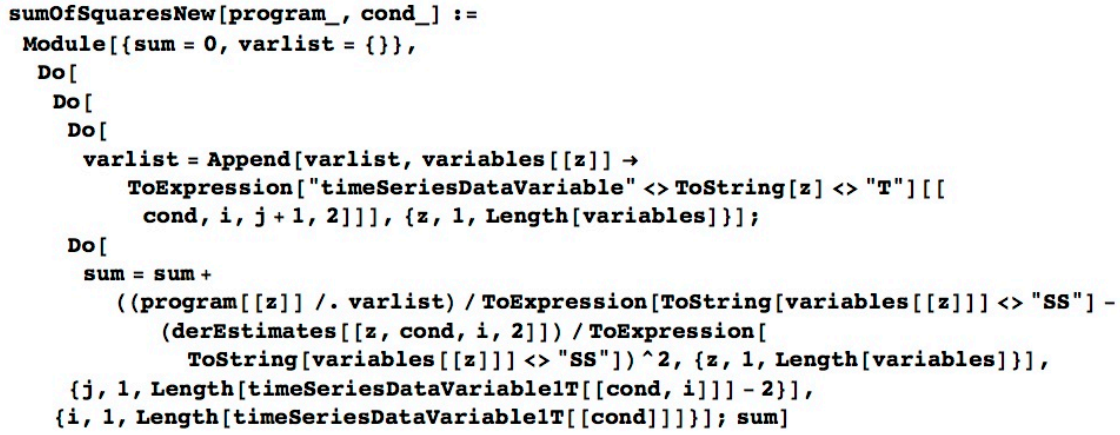

The "sumOfSquaresNew2" function will calculate the sum of squared differences between model data and experimental data.

sumofSquaresNew2 [expdata_, mdata_] :=

Module $[\{\operatorname{sum}=0\}, \operatorname{Do}[\operatorname{Do}[\operatorname{sum}=\operatorname{sum}+(\operatorname{expdata}[[i, j+1,2]]-\operatorname{mdata}[[j, 2]]) \wedge 2$,

$\{j, 1$, Length [mdata $]\},\{i, 1$, Length [expdata] $\}]$; sum]

\section{Execution of the Algorithm}

The command below will exectute the evolutionary algorithm to optimize a mass balance for the variable under consideration.

ea [] ; 